\title{
Oxidative C-H Homocoupling of Push-Pull Benzothiazoles: An Atom-Economical Route to Highly Emissive Quadrupolar Arylamine-Functionalized 2,2'-Bibenzothiazoles with Enhanced Two-Photon Absorption
}

\author{
Patrik Osuský, ${ }^{a}$ Jela Nociarová, ${ }^{a}$ Maroš Smoliček, ${ }^{a}$ Róbert Gyepes, ${ }^{b}$ Dimitris Georgiou, ${ }^{c}$ \\ Ioannis Polyzos, ${ }^{c}$ Mihalis Fakis, ${ }^{c}$ Peter Hrobárik ${ }^{a, d^{*}}$ \\ ${ }^{a}$ Department of Inorganic Chemistry, Faculty of Natural Sciences, \\ Comenius University, SK-84215 Bratislava, Slovakia \\ ${ }^{b}$ Department of Inorganic Chemistry, Faculty of Science, Charles University, \\ Hlavova 2038/8, CZ-12843 Prague, Czech Republic \\ ${ }^{c}$ Department of Physics, University of Patras, GR-26504 Patras, Greece \\ ${ }^{d}$ Laboratory for Advanced Materials, Comenius University Science Park, \\ Ilkovičova 8, SK-84215 Bratislava, Slovakia
}

peter.hrobarik@uniba.sk

\section{Supporting Information}




\section{Table of Contents}

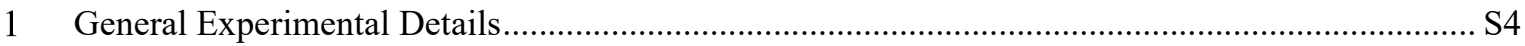

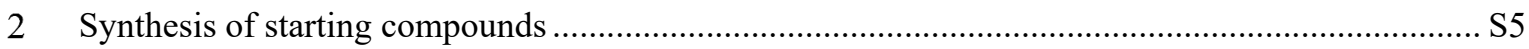

2.1 Synthesis of 6-iodobenzothiazole from benzothiazol-2(3H)-one .......................................... S5

2.1.1 Iodination of benzothiazol-2(3H)-one with $N$-iodosuccinimide....................................... S5

2.1.2 Chlorination of 6-iodobenzothiazol-2(3H)-one ............................................................ S5

2.1.3 Hydrodechlorination of 2-chloro-6-iodobenzothiazole ……........................................... S6

2.2 Synthesis of benzothiazol-6-amine from benzothiazole ........................................................... S7

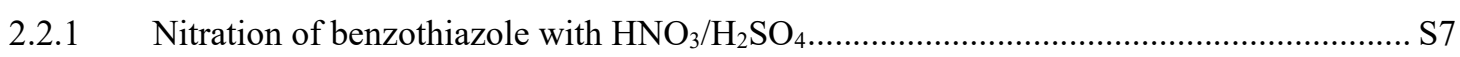

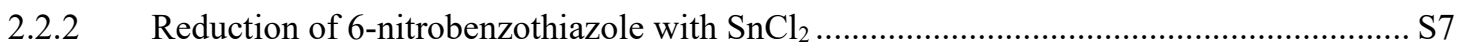

2.3 Synthesis of arylamine-functionalized phenylacetylenes ....................................................... S8

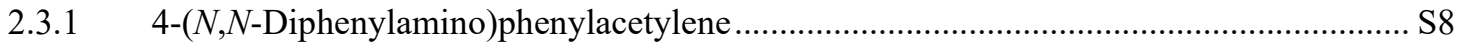

2.3.2 4-[N,N-Bis(4-methoxyphenyl)amino]phenylacetylene.................................................... $\mathrm{S} 9$

2.4 Synthesis of arylamine-functionalized phenylboronic acids ............................................... S11

2.4.1 4-( $N, N$-Diphenylamino)phenylboronic acid .............................................................. S1

2.4.2 4-[N,N-Bis(4-methoxyphenyl)amino]phenylboronic acid .............................................. S11

3 Synthesis of arylamine-functionalized 2-H-benzothiazoles ......................................................... S13

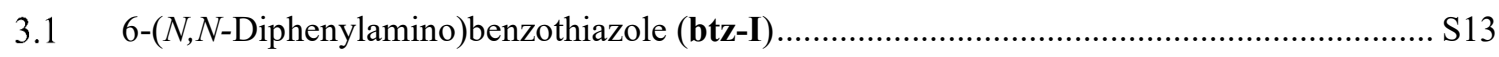

3.1.1 Method A - Arylation of benzothiazol-6-amine with trifenylsulfonium triflate.............. S13

3.1.2 Method B - Arylation of benzothiazol-6-amine with 4-fluoronitrobenzene ................... S14

3.2 Buchwald-Hartwig cross-coupling reactions...................................................................... S17

3.2.1 Benzothiazol-6-amine with bromobenzene and $\mathrm{Cs}_{2} \mathrm{CO}_{3}$ as a base ............................... S17

3.2.2 Benzothiazol-6-amine with bromobenzene and $t \mathrm{BuOK}$ as a base.................................. $\mathrm{S} 17$

3.3 6-[4-(N,N-Diphenylamino)phenyl]benzothiazole (btz-II) …………………...................... S19

$3.4 \quad 6-\{4-[N, N$-Bis(4-methoxyphenyl)amino]phenyl $\}$ benzothiazole (btz-III) ............................. S19

3.5 6-[4-( $N, N$-Diphenylamino)phenylethynyl]benzothiazole (btz-IV) ...................................... S20

3.6 6- $\{4-[N, N$-Bis(4-methoxyphenyl)amino]phenylethynyl $\}$ benzothiazole (btz-V) ................... S20

3.7 (E)-6-[4-(N,N-Diphenylamino)phenylethenyl]benzothiazole (btz-VI) ................................ S21

4 Oxidative homocoupling of 2-H-benzothiazoles ...................................................................... S22

4.1 Synthesis of $2,2^{\prime}$-bibenzothiazole.............................................................................. S22

4.2 Homocoupling of arylamine-functionalized 2-H-benzothiazoles - general procedure ........... S23

4.2.1 6,6'-Bis( $N, N$-diphenylamino)-2,2'-bibenzothiazole (bisbtz-I) ..................................... S23

4.2.2 6,6'-Bis[4-( $N, N$-diphenylamino)phenyl]-2,2'-bibenzothiazole (bisbtz-II)..................... S23

4.2.3 6,6'-Bis $\{4-[N, N$-bis(4-methoxyphenyl)amino]phenyl $\}$-2,2'-bibenzothiazole

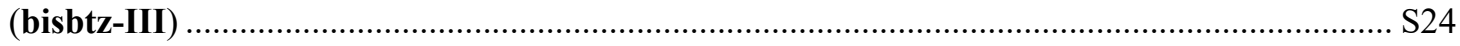

4.2.4 6,6'-Bis[4-( $N, N$-diphenylamino)phenylethynyl]-2,2'-bibenzothiazole (bisbtz-IV) ....... S24

4.2.5 6,6'-Bis $\left\{4-[N, N\right.$-Bis(4-methoxyphenyl)amino]phenylethynyl $\}-2,2^{\prime}$ '-bibenzothiazole

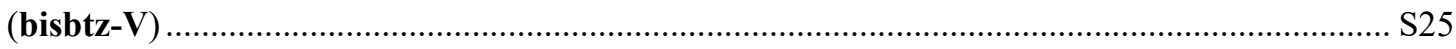


4.2.6 (E,E)-6,6'-[4-(N,N-Diphenylamino)phenylethenyl]-2,2'-bibenzothiazole

(bisbtz-VI) S25

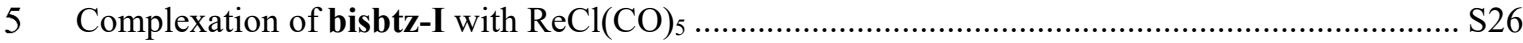

6 NMR spectra of key intermediates and target 2,2'-bibenzothiazoles ............................................. S27

7 Synthesis of $(E, E)-4,4$ '-[4-( $N, N$-diphenylamino)phenylethenyl]-2,2'-bipyridine ............................ S54

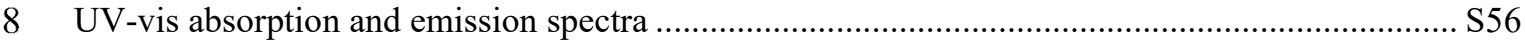

9 Fluorescence quantum yields measurements and TCSPC spectroscopy ....................................... S60

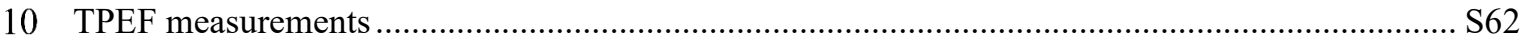

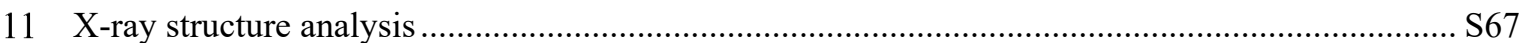

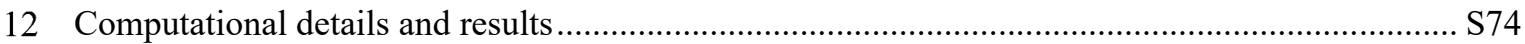

13 Cartesian coordinates of pertinent DFT optimized structures ................................................... S80

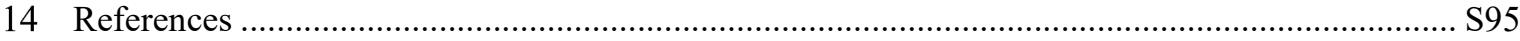




\section{General Experimental Details}

All chemicals were used as received from commercial suppliers without purification, if not mentioned otherwise. Benzothiazole, benzothiazol-2(3H)-one, diphenyl sulfide, trimethylsilyl acetylene, aniline, trimethyl borate, $\mathrm{Pd}\left(\mathrm{PPh}_{3}\right)_{2} \mathrm{Cl}_{2}, \mathrm{Pd}\left(\mathrm{PPh}_{3}\right)_{4}, \mathrm{Pd}(\mathrm{OAc})_{2}, \mathrm{Pd}_{2}(\mathrm{dba})_{3}$ and $\mathrm{CuI}$ were purchased from TCI Chemicals, $N$-iodosuccinimide, 4-bromotriphenylamine, 4-iodoanisole, 4-fluoronitrobenzene, bromobenzene, iodobenzene, BINAP, trifluoromethanesulfonic (triflic) acid and potassium tert-butoxide from Fluorochem Ltd., $\mathrm{Cu}(\mathrm{OAc})_{2}, \mathrm{Cs}_{2} \mathrm{CO}_{3}, \mathrm{POCl}_{3}, \mathrm{KI}, \mathrm{H}_{3} \mathrm{PO}_{2}$, $\mathrm{ReCl}(\mathrm{CO})_{5}, n$-BuLi, phenantroline, 3-chloroperoxybenzoic acid and all organic solvents from Acros Organics. Solvents for Pd-catalysed cross-coupling reactions were purified prior to the synthesis: tetrahydrofuran was distilled from $\mathrm{Na}$ /benzophenone, triethylamine from $\mathrm{CaH}_{2}$. DMSO was dried using $4 \AA$ molecular sieves. Commercially available $\mathrm{Cu}(\mathrm{OAc})_{2}$ was dried under reduced pressure at $155^{\circ} \mathrm{C}$ for 3 hours.

NMR spectra were recorded with Varian NMR System $600\left(600 \mathrm{MHz}\right.$ for ${ }^{1} \mathrm{H}$ and $151 \mathrm{MHz}$ for ${ }^{13} \mathrm{C}$ ) and Varian NMR System ${ }^{\mathrm{TM}} 300\left(300 \mathrm{MHz}\right.$ for ${ }^{1} \mathrm{H}$ and $75 \mathrm{MHz}$ for $\left.{ }^{13} \mathrm{C}\right)$ instruments. ${ }^{1} \mathrm{H}$ and ${ }^{13} \mathrm{C}$ chemical shifts $(\delta)$ are given relative to tetramethylsilane, samples were prepared in $\mathrm{CDCl}_{3}$ $\left({ }^{1} \mathrm{H} \delta 7.26,{ }^{13} \mathrm{C} \delta 77.0 \mathrm{ppm}\right)$ or DMSO- $d_{6}\left({ }^{1} \mathrm{H} \delta 2.50,{ }^{13} \mathrm{C} \delta 39.5 \mathrm{ppm}\right) .{ }^{15} \mathrm{~N}$ chemical shifts were measured indirectly, via ${ }^{1} \mathrm{H}-{ }^{15} \mathrm{~N}$ long range correlation on a VARIAN INOVA $600 \mathrm{MHz} \mathrm{NMR}$ spectrometer working at $599.78\left({ }^{1} \mathrm{H}\right)$ and $60.78 \mathrm{MHz}\left({ }^{15} \mathrm{~N}\right)$ equipped with Varian $5 \mathrm{~mm}$ triple resonance probe ${ }^{1} \mathrm{H}\left\{{ }^{13} \mathrm{C} /{ }^{15} \mathrm{~N}\right\}$ equipped with an Z-gradient coil. Unified chemical shift scale was used for ${ }^{15} \mathrm{~N}$ with nitromethane as a secondary standard $(\delta=0.0 \mathrm{ppm} ; \Xi=10.1367670) .{ }^{[1]}$

UV-vis spectra were measured on Hewlett Packard 8452A Diode Array spectrophotometer and fluorescence spectra were measured on Shimadzu RF 6000 Spectrofluorophotometer.

IR spectra were measured on Agilent Technologies Cary 630 FTIR. Melting points were measured on Büchi Melting Point M-565.

Elemental analysis was performed on Vario Micro Cube.

Column chromatography was performed with Merck Silica Gel $60(0.040-0.063 \mathrm{~mm})$ and Merck Alumina 90 (neutral), respectively. Thin layer chromatography (TLC) was performed with Merck plates (Silica Gel 60, F-254). Compounds were detected at $254 \mathrm{~nm}$ and $365 \mathrm{~nm}$, respectively. 


\section{Synthesis of starting compounds}

\subsection{Synthesis of 6-iodobenzothiazole from benzothiazol-2(3H)-one}

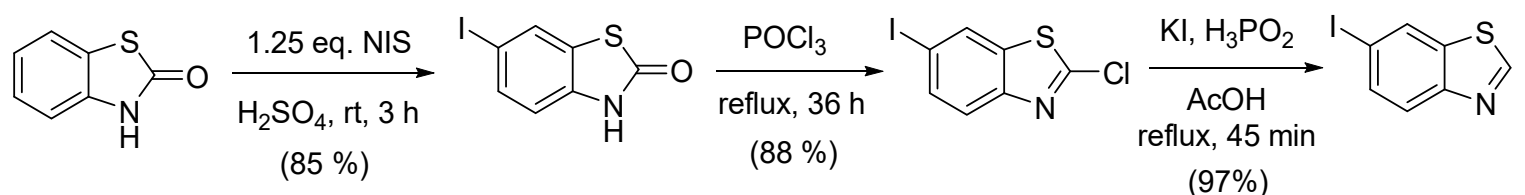

\subsubsection{Iodination of benzothiazol-2(3H)-one with $\mathrm{N}$-iodosuccinimide}

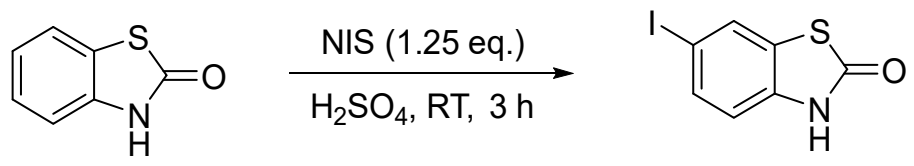

Benzothiazol-2(3H)-one (10.0 g, $66 \mathrm{mmol})$ was dissolved in the sulfuric acid $(200 \mathrm{~mL})$ diluted with $10 \mathrm{~mL}$ of deionized water. $N$-Iodosuccinimide $(18.6 \mathrm{~g}, 83 \mathrm{mmol})$ was added portionwise over $5 \mathrm{~min}$ at $0{ }^{\circ} \mathrm{C}$. The reaction mixture was stirred at room temperature for 3 hours and then poured onto ice $(600 \mathrm{~g})$. The solid was filtered off, washed with water until neutral reaction of the filtrate, then with $5 \%$ aqueous solution of $\mathrm{Na}_{2} \mathrm{~S}_{2} \mathrm{O}_{5}$, saturated aq. solution of $\mathrm{NaHCO}_{3}$ and deionized water. The solid was dried under reduced pressure to obtain 6-iodobenzothiazol-2(3H)-one as a brownish solid (15.6 g, $85 \%) . \boldsymbol{R}_{\mathbf{f}}=0.13\left(\mathrm{SiO}_{2}\right.$, hexanes/ethyl acetate $\left.=6: 1\right) ; \mathbf{M p ~} 222-223{ }^{\circ} \mathrm{C}$ (lit. $\left.{ }^{[2]} \mathrm{mp} 225-226{ }^{\circ} \mathrm{C}\right) ;{ }^{1} \mathbf{H}$ NMR $\left(600 \mathrm{MHz}, \mathrm{CDCl}_{3}\right) \delta 9.18(\mathrm{~s}, 1 \mathrm{H}), 7.71(\mathrm{~d}, J=1.2 \mathrm{~Hz}, 1 \mathrm{H})$, $7.58(\mathrm{dd}, J=8.4,1.6 \mathrm{~Hz}, 1 \mathrm{H}), 6.90(\mathrm{~d}, J=8.4 \mathrm{~Hz}, 1 \mathrm{H}) .{ }^{13} \mathbf{C ~ N M R}\left(151 \mathrm{MHz}, \mathrm{CDCl}_{3}\right) \delta 171.1$, $135.3,134.4,130.8,126.1,113.0,85.3$.

\subsubsection{Chlorination of 6-iodobenzothiazol-2(3H)-one}

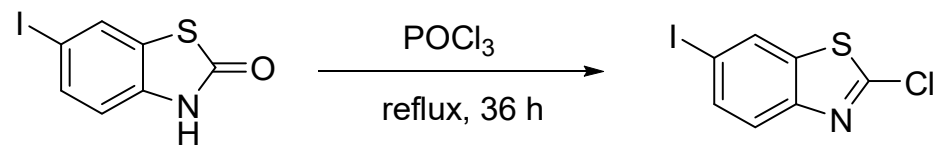

$\mathrm{POCl}_{3}(80 \mathrm{~mL})$ was added via syringe into an argon-flushed round-bottom flask equipped with a condenser and containing 6-iodobenzothiazol-2(3H)-one $(10.0 \mathrm{~g}, 36 \mathrm{mmol})$. The reaction mixture was heated to reflux for $36 \mathrm{~h}$. After consumption of the starting material (monitored by TLC), the mixture was cooled to room temperature and then added into $500 \mathrm{~mL}$ of vigorously stirred water in small portions over $30 \mathrm{~min}$ (the temperature throughout the addition was maintained under 40 $\left.{ }^{\circ} \mathrm{C}\right)$. The precipitate formed was collected by filtration, dissolved in chloroform $(250 \mathrm{~mL})$, dried over $\mathrm{Na}_{2} \mathrm{SO}_{4}$ and concentrated in vacuo to obtain 2-chloro-6-iodo-benzothiazole as a white solid $(9.33 \mathrm{~g}, 88 \%) . \boldsymbol{R}_{\mathbf{f}}=0.71\left(\mathrm{SiO}_{2}\right.$, hexanes/ethyl acetate $\left.=6: 1\right) ; \mathbf{M p} 134-136{ }^{\circ} \mathrm{C}\left(\right.$ lit. $^{[3]} \mathrm{mp} 135-$ 
$\left.137^{\circ} \mathrm{C}\right) .{ }^{1} \mathbf{H}$ NMR $\left(600 \mathrm{MHz}, \mathrm{CDCl}_{3}\right) \delta 8.11(\mathrm{~d}, J=1.4 \mathrm{~Hz}, 1 \mathrm{H}), 7.77(\mathrm{dd}, J=8.6,1.7 \mathrm{~Hz}, 1 \mathrm{H})$, $7.67(\mathrm{~d}, J=8.6 \mathrm{~Hz}, 1 \mathrm{H}) ;{ }^{13} \mathbf{C} \mathbf{N M R}\left(151 \mathrm{MHz}, \mathrm{CDCl}_{3}\right) \delta 153.8,150.3,137.9,135.9,129.5,124.3$, 90.1 .

\subsubsection{Hydrodechlorination of 2-chloro-6-iodobenzothiazole}

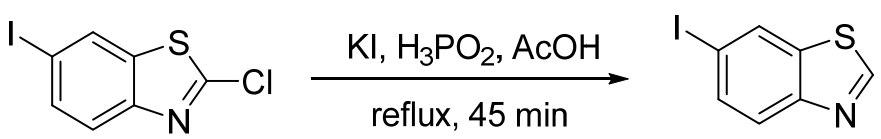

2-Chloro-6-iodobenzothiazole $(9.315 \mathrm{~g}, 31.5 \mathrm{mmol})$ was dissolved in acetic acid $(150 \mathrm{~mL})$ and $\mathrm{KI}(15.7 \mathrm{~g}, 95 \mathrm{mmol})$ and $\mathrm{H}_{3} \mathrm{PO}_{2}(20 \mathrm{~mL})$ were added. The mixture was heated to $80{ }^{\circ} \mathrm{C}$ for 45 min. After cooling to room temperature, the mixture was poured onto ice, neutralized with aqueous $\mathrm{NaOH}(20 \%)$ and extracted with dichloromethane $(3 \times 100 \mathrm{~mL})$. Combined organic layers were washed with $5 \%$ aqueous solution of $\mathrm{Na}_{2} \mathrm{~S}_{2} \mathrm{O}_{5}$, water, brine and dried over $\mathrm{Na}_{2} \mathrm{SO}_{4}$ to afford 6iodobenzothiazole as a white solid $(7.94 \mathrm{~g})$ in $97 \%$ yield. $\boldsymbol{R}_{\mathbf{f}}=0.37\left(\mathrm{SiO}_{2}\right.$, hexanes/ethyl acetate $=6: 1) ; \mathbf{M p} 77-79{ }^{\circ} \mathrm{C}\left(\right.$ lit. $\left.^{[4]} \mathrm{mp} 78-80{ }^{\circ} \mathrm{C}\right) ;{ }^{1} \mathbf{H} \mathbf{~ N M R}\left(600 \mathrm{MHz}, \mathrm{CDCl}_{3}\right) \delta 8.91(\mathrm{~s}, 1 \mathrm{H}), 8.30$ $(\mathrm{d}, J=1.2 \mathrm{~Hz}, 1 \mathrm{H}), 7.87(\mathrm{~d}, J=8.6 \mathrm{~Hz}, 1 \mathrm{H}), 7.79(\mathrm{dd}, J=8.6,1.5 \mathrm{~Hz}, 1 \mathrm{H}) .{ }^{13} \mathbf{C} \mathbf{N M R}(151 \mathrm{MHz}$, $\left.\mathrm{CDCl}_{3}\right) \delta 154.3,152.7,135.9,135.3,130.4,125.1,90.1$. 


\subsection{Synthesis of benzothiazol-6-amine from benzothiazole}

\subsubsection{Nitration of benzothiazole with $\mathrm{HNO}_{3} / \mathrm{H}_{2} \mathrm{SO}_{4}$}

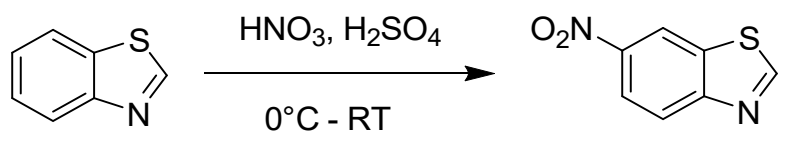

Benzothiazole $(18.2 \mathrm{~mL}, 0.17 \mathrm{~mol})$ was dissolved in concentrated $\mathrm{H}_{2} \mathrm{SO}_{4}(46 \mathrm{~mL})$. The solution was cooled to $0{ }^{\circ} \mathrm{C}$ and fuming $\mathrm{HNO}_{3}(18.2 \mathrm{~mL})$ was added dropwise at such a rate to keep the temperature below $5{ }^{\circ} \mathrm{C}$ throughout the addition. After the addition was completed, the reaction mixture was stirred under cooling for $15 \mathrm{~min}$ and then at room temperature for an additional $2 \mathrm{~h}$. Subsequently, the mixture was poured into ice-cold water $(400 \mathrm{~mL})$. The precipitate was collected by filtration, washed with cold water until the $\mathrm{pH}$ was neutral, and then crystallized from ethanol to give 6-nitrobenzothiazole as a white solid in $65 \%$ yield $(20.0 \mathrm{~g})$. Mp $176-177^{\circ} \mathrm{C}$ (lit. ${ }^{[4]} \mathrm{mp}$ $\left.176-177{ }^{\circ} \mathrm{C}\right) ;{ }^{1} \mathbf{H}$ NMR $\left(300 \mathrm{MHz}, \mathrm{CDCl}_{3}\right) \delta 9.28(\mathrm{~s}, 1 \mathrm{H}), 8.93(\mathrm{~d}, J=2.4 \mathrm{~Hz}, 1 \mathrm{H}), 8.41(\mathrm{dd}, J$ $=9.0,2.3 \mathrm{~Hz}, 1 \mathrm{H}), 8.26(\mathrm{~d}, J=9.0 \mathrm{~Hz}, 1 \mathrm{H})$.

\subsubsection{Reduction of 6-nitrobenzothiazole with $\mathrm{SnCl}_{2}$}

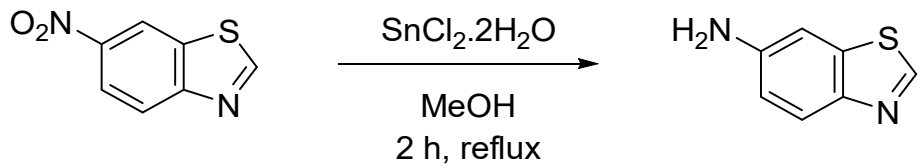

6-Nitrobenzothiazole $(20.0 \mathrm{~g}, 111 \mathrm{mmol}, 1.0 \mathrm{eq})$ was suspended in a mixture of $\mathrm{CHCl}_{3}(200 \mathrm{~mL})$ and $\mathrm{MeOH}(100 \mathrm{~mL}) . \mathrm{SnCl}_{2} \cdot 2 \mathrm{H}_{2} \mathrm{O}(90 \mathrm{~g}, 398.9 \mathrm{mmol}, 3.6 \mathrm{eq})$ was added in one portion followed by $\mathrm{MeOH}(100 \mathrm{~mL})$. The reaction mixture was heated to reflux over $3 \mathrm{~h}$ under an inert atmosphere. Then the reaction mixture was cooled to $\mathrm{rt}$, poured to a crushed ice $(1.5 \mathrm{~L})$, basified with $10 \mathrm{M}$ $\mathrm{NaOH}(500 \mathrm{~mL})$ and DCM $(300 \mathrm{~mL})$ was added. The mixture was stirred $30 \mathrm{~min}$ at $\mathrm{rt}$ and after separation of phases, the aqueous phase was extracted with another DCM $(2 \times 250 \mathrm{~mL})$. Combined organic (DCM) phases were washed with brine, dried with anhydrous $\mathrm{Na}_{2} \mathrm{SO}_{4}$, filtered and solvent removed under reduced pressure. The product was isolated as a yellow solid (15.3 g, $92 \%$ yield). Mp $86-87^{\circ} \mathrm{C}\left(\right.$ lit. $\left.^{[4]} \mathrm{mp} 86-87^{\circ} \mathrm{C}\right) ;{ }^{1} \mathbf{H}$ NMR $(600 \mathrm{MHz}$, DMSO-d 6 ) $\delta 8.87$ (s, 1H), $7.71(\mathrm{~d}, J$ $=8.7 \mathrm{~Hz}, 1 \mathrm{H}), 7.12(\mathrm{~d}, J=2.2 \mathrm{~Hz}, 1 \mathrm{H}), 6.80(\mathrm{dd}, J=8.7,2.2 \mathrm{~Hz}, 1 \mathrm{H}), 5.40(\mathrm{~s}, 2 \mathrm{H})$. 


\subsection{Synthesis of arylamine-functionalized phenylacetylenes}

\subsubsection{4-( $N, N$-Diphenylamino)phenylacetylene}

\subsubsection{Synthesis of N, N-diphenyl-4-[(trimethylsilyl)ethynyl]aniline}
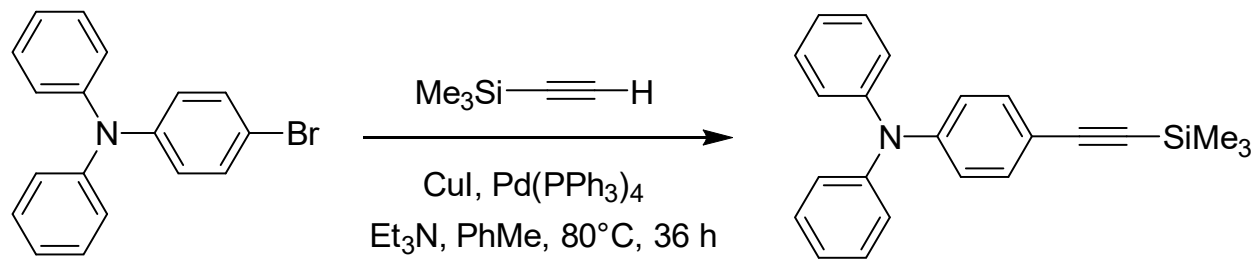

4-Bromotriphenylamine (10 g, $30.8 \mathrm{mmol})$, $\mathrm{CuI}$ (295 mg, $1.54 \mathrm{mmol})$ and $\mathrm{Pd}\left(\mathrm{PPh}_{3}\right)_{4}(1.424 \mathrm{~g}$, $1.232 \mathrm{mmol})$ were dissolved in dry toluene $(50 \mathrm{~mL})$ under inert atmosphere and dry triethylamine $(52 \mathrm{~mL})$ was added. Then trimethylsilylacetylene $(6.6 \mathrm{~mL}, 46.2 \mathrm{mmol})$ was added and the mixture was heated to $80^{\circ} \mathrm{C}$ (in an oil bath) under inert atmosphere for 36 hours. Subsequently, the reaction mixture was cooled to $\mathrm{rt}$ and concentrated under reduced pressure. The solid residue was purified by column chromatography on silica gel (eluent: hexanes) to give the desired product as an orange solid $(6.17 \mathrm{~g})$ in $59 \%$ yield. ${ }^{1} \mathbf{H}$ NMR $\left(600 \mathrm{MHz}, \mathrm{CDCl}_{3}\right) \delta 7.32-7.29(\mathrm{~m}, 2 \mathrm{H}), 7.26(\mathrm{t}, J=7.9$ $\mathrm{Hz}, 4 \mathrm{H}), 7.10-7.07(\mathrm{~m}, 4 \mathrm{H}), 7.06-7.03(\mathrm{~m}, 2 \mathrm{H}), 6.95$ (d, J=8.7 Hz, 2H), 0.23 (s, 9H). ${ }^{1} \mathrm{H}$ NMR shifts match those reported for this compound in ref. [5].

\subsubsection{Synthesis of 4-(N,N-diphenylamino)phenylacetylene}

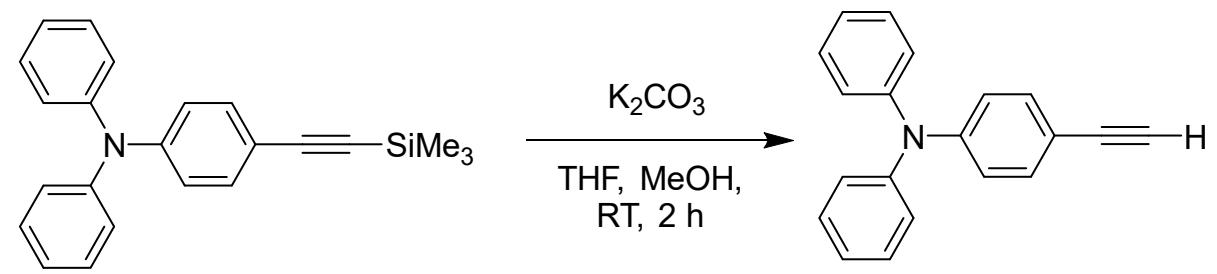

$N, N$-Diphenyl-4-[(trimethylsilyl)ethynyl]aniline $(6.17 \mathrm{~g}, 18.1 \mathrm{mmol})$ and potassium carbonate (24.96 g, $18.1 \mathrm{mmol})$ were dissolved in $\mathrm{MeOH}(100 \mathrm{~mL})$ and THF $(100 \mathrm{~mL})$ and the mixture was stirred at room temperature for 2 hours. Then, insoluble $\mathrm{K}_{2} \mathrm{CO}_{3}$ was filtered off and organic solvents evaporated under reduced pressure. Purification by column chromatography on silica gel (eluent: hexanes / ethyl acetate 9:1) afforded the desired arylacetylene as a yellow solid (3.02 $\mathrm{g}$ ) in $62 \%$ yield. $\boldsymbol{R}_{\mathbf{f}} 0.48\left(\mathrm{SiO}_{2}\right.$, hexanes $\left./ \mathrm{CHCl}_{3}=4: 1\right)$; $\mathbf{M p ~} 80-82{ }^{\circ} \mathrm{C}\left(\right.$ lit. $\left.{ }^{[4]} 80-82{ }^{\circ} \mathrm{C}\right){ }^{1} \mathbf{H ~} \mathbf{~ N M R}$ $\left(600 \mathrm{MHz} \mathrm{CDCl}_{3}\right) \delta 7.34-7.32(\mathrm{~m}, 2 \mathrm{H}), 7.29$ - $7.25(\mathrm{~m}, 4 \mathrm{H}), 7.11-7.09(\mathrm{~m}, 4 \mathrm{H}), 7.07$ - 7.04 $(\mathrm{m}, 2 \mathrm{H}), 6.97-6.95(\mathrm{~m}, 2 \mathrm{H}), 3.02(\mathrm{~s}, 1 \mathrm{H}) .{ }^{1} \mathrm{H}$ NMR shifts match those reported previously in ref. [4]. 


\subsubsection{4-[N,N-Bis(4-methoxyphenyl)amino]phenylacetylene}

\subsubsection{Synthesis of $\mathrm{N}$, $\mathrm{N}$-bis(4-methoxyphenyl)aniline}

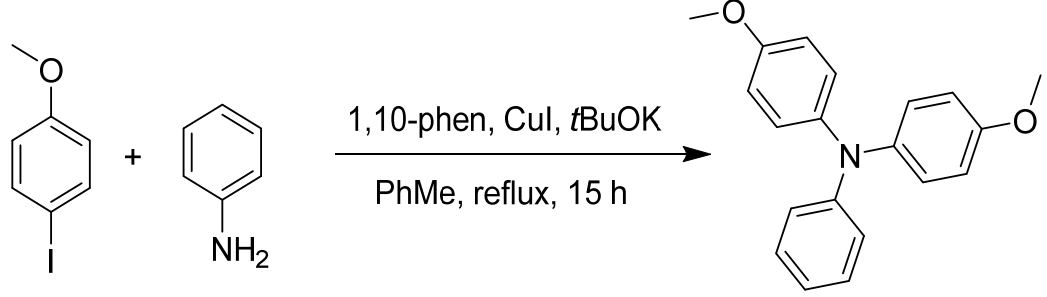

1-Iodo-4-methoxybenzene (20 g, $85.46 \mathrm{mmol})$, aniline (3.8 mL, $40.7 \mathrm{mmol}), \mathrm{CuI}$ (1.55 g, 8.14 mmol), 1,10-phenantroline (1.47 g, $8.14 \mathrm{mmol})$ and tert-BuOK (36.5 g, $325.6 \mathrm{mmol})$ were dissolved in dry toluene $(250 \mathrm{~mL})$ under argon atmosphere. Reaction mixture was heated to reflux in an oil bath for 16 hours. After cooling to rt, the reaction mixture was quenched with water (250 $\mathrm{mL})$ and extracted with dichloromethane $(3 \times 250 \mathrm{~mL})$. Organic phase was washed with brine (200 $\mathrm{mL}$ ), dried over $\mathrm{Na}_{2} \mathrm{SO}_{4}$ and concentrated under reduced pressure. The crude solid was purified by column chromatography on silica gel (eluent: hexanes / dichloromethane 1:1) affording the desired product as a yellow solid (4.9 g) in $40 \%$ yield. $\boldsymbol{R}_{\mathbf{f}} 0.2\left(\mathrm{SiO}_{2}\right.$, hexanes/DCM=1:1); Mp 105 $-106{ }^{\circ} \mathrm{C}$ (lit. $\left.{ }^{[6]} 104-106{ }^{\circ} \mathrm{C}\right) .{ }^{1} \mathbf{H}$ NMR $\left(600 \mathrm{MHz}, \mathrm{CDCl}_{3}\right) \delta 7.16$ (dd, $\left.J=8.6,7.3 \mathrm{~Hz}, 2 \mathrm{H}\right), 7.03$ $(\mathrm{d}, J=8.8 \mathrm{~Hz}, 4 \mathrm{H}), 6.95-6.91(\mathrm{~m}, 2 \mathrm{H}), 6.87-6.83(\mathrm{~m}, 1 \mathrm{H}), 6.81(\mathrm{~d}, J=8.9 \mathrm{~Hz}, 4 \mathrm{H}), 3.78(\mathrm{~s}$, $6 \mathrm{H})$.

\subsubsection{Synthesis of 4-iodo-N, N-bis(4-methoxyphenyl)aniline}

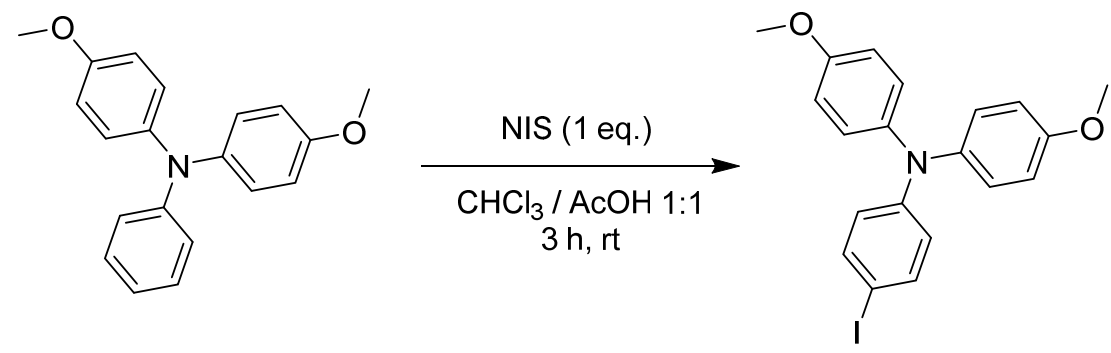

$N, N$-Bis(4-methoxyphenyl)aniline $(6.40 \mathrm{~g}, 20.96 \mathrm{mmol})$ was dissolved in a mixture of chloroform $(25 \mathrm{~mL})$ and acetic acid $(25 \mathrm{~mL})$. Then $N$-iodosuccinimide $(4.72 \mathrm{~g}, 20.96 \mathrm{mmol})$ was added portionwise to reaction mixture. The reaction mixture was stirred at room temperature for 3 hours, then quenched with water $(50 \mathrm{~mL})$ and extracted using chloroform $(3 \times 30 \mathrm{~mL})$. Organic phase was dried over $\mathrm{Na}_{2} \mathrm{SO}_{4}$ and solvents removed under reduced pressure. The solid residue was purified by column chromatography on silica gel (eluent: hexanes / dichloromethane 1:1) affording the product as a yellow solid ( $7.60 \mathrm{~g})$ in $84 \%$ yield. $\boldsymbol{R}_{\mathbf{f}} 0.23\left(\mathrm{SiO}_{2}\right.$, hexanes/DCM=1:1); Mp 113-115 ${ }^{\circ} \mathrm{C}$ (lit. $\left.{ }^{[7]} 114-116{ }^{\circ} \mathrm{C}\right){ }^{1} \mathbf{H}$ NMR $\left(600 \mathrm{MHz}, \mathrm{CDCl}_{3}\right) \delta 7.40(\mathrm{~d}, J=8.8 \mathrm{~Hz}, 2 \mathrm{H}), 7.05-7.01$ (m, $4 \mathrm{H}), 6.84-6.81(\mathrm{~m}, 4 \mathrm{H}), 6.67(\mathrm{~d}, J=8.9 \mathrm{~Hz}, 2 \mathrm{H}), 3.79(\mathrm{~s}, 6 \mathrm{H})$. 


\subsubsection{Synthesis of $\mathrm{N}, \mathrm{N}$-bis(4-methoxyphenyl)-4-[(trimethylsilyl)ethynyl]aniline}

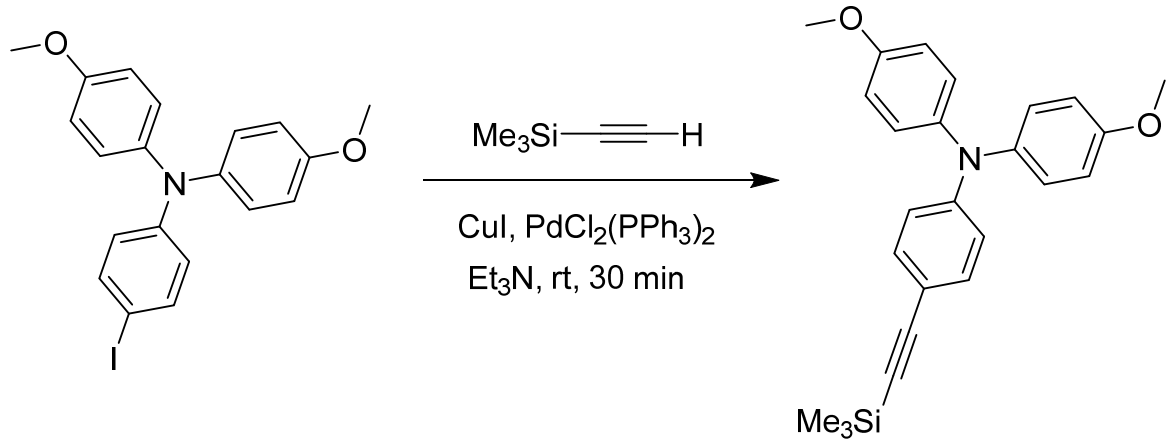

4-Iodo- $N, N$-bis(4-methoxyphenyl)aniline (0.5 g, $1.16 \mathrm{mmol}), \mathrm{CuI}(11 \mathrm{mg}, 0.058 \mathrm{mmol})$ and $\mathrm{PdCl}_{2}\left(\mathrm{PPh}_{3}\right)_{2}(41 \mathrm{mg}, 0.058 \mathrm{mmol})$ were dissolved in dry triethylamine $(7 \mathrm{~mL})$ under argon. Then trimethylsylilacetylene $(0.25 \mathrm{~mL}, 1.74 \mathrm{mmol})$ was added dropwise. The reaction mixture was stirred under inert atmosphere for 30 minutes. After this time, the mixture was concentrated under reduced pressure, the solid residue suspended in chloroform $(10 \mathrm{~mL})$ and filtered through a short pad of silica. The desired product was obtained upon evaporation of chloroform under reduced pressure as a yellow solid (451 mg) in $97 \%$ yield. ${ }^{1} \mathbf{H}$ NMR $\left(600 \mathrm{MHz}, \mathrm{CDCl}_{3}\right) \delta 7.24(\mathrm{~d}, J=8.9$ $\mathrm{Hz}, 2 \mathrm{H}), 7.04$ (d, $J=9.0 \mathrm{~Hz}, 4 \mathrm{H}), 6.83$ (d, $J=9.0 \mathrm{~Hz}, 4 \mathrm{H}), 6.79$ (d, $J=8.9 \mathrm{~Hz}, 2 \mathrm{H}), 3.79$ (s, 6H), $0.22(\mathrm{~s}, 9 \mathrm{H}) .{ }^{1} \mathrm{H}$ NMR shifts match those reported for this compound in ref. [8].

\subsubsection{Synthesis of 4-[N, N-bis(4-methoxyphenyl)amino]phenylacetylene}
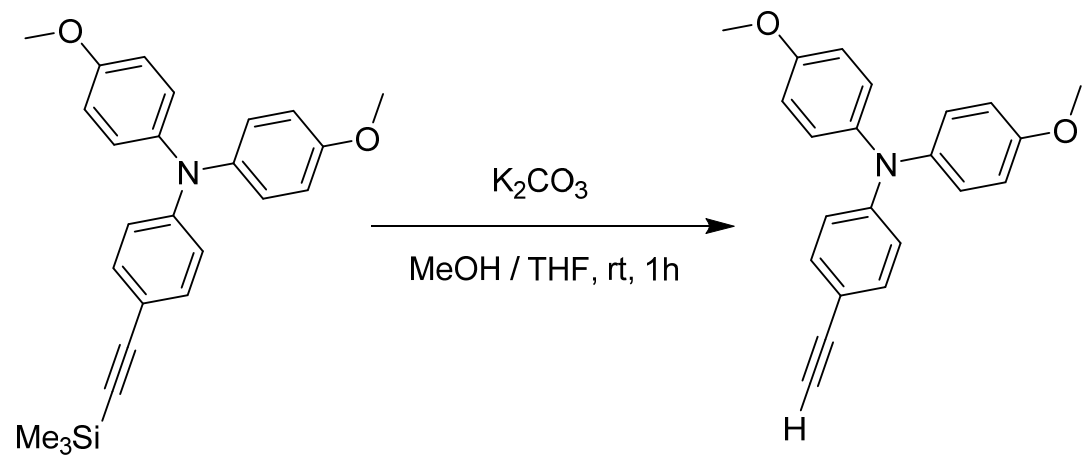

$N, N$-bis(4-methoxyphenyl)-4-[(trimethylsilyl)ethynyl]aniline $(451 \mathrm{mg}, 1.123 \mathrm{mmol})$ and potassium carbonate $(233 \mathrm{mg}, 1.686 \mathrm{mmol})$ were dissolved in a mixture of methanol $(10 \mathrm{~mL})$ and tetrahydrofuran $(5 \mathrm{~mL})$. The reaction mixture was stirred at room temperature for 1 hour, then filtered and concentrated under reduced pressure. The solid residue was purified by column chromatography on silica gel (eluent: hexanes / dichloromethane 1:1) affording the desired arylacetylene as a yellow solid $(278 \mathrm{mg})$ in $75 \%$ yield. $\boldsymbol{R}_{\mathbf{f}} 0.3\left(\mathrm{SiO}_{2}\right.$, hexanes/DCM=1:1); ${ }^{1} \mathbf{H}$ NMR $\left(600 \mathrm{MHz}, \mathrm{CDCl}_{3}\right) \delta 7.26(\mathrm{~s}, 2 \mathrm{H}), 7.07-7.04(\mathrm{~m}, 4 \mathrm{H}), 6.85-6.82(\mathrm{~m}, 4 \mathrm{H}), 6.80(\mathrm{~d}, J=$ $8.7 \mathrm{~Hz}, 2 \mathrm{H}), 3.79(\mathrm{~s}, 6 \mathrm{H}), 2.98(\mathrm{~s}, 1 \mathrm{H}) .{ }^{1} \mathrm{H}$ NMR shifts match those reported for this compound in ref. [8]. 


\subsection{Synthesis of arylamine-functionalized phenylboronic acids}

\subsubsection{4-( $N, N$-Diphenylamino)phenylboronic acid}
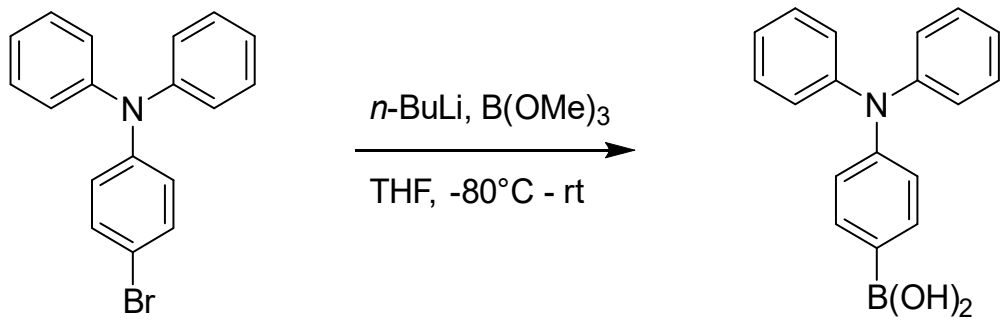

4-Bromotriphenylamine (10.0 g, $30.8 \mathrm{mmol})$ was dissolved in dry THF (60 mL). The mixture was cooled to $-80{ }^{\circ} \mathrm{C}$ and then $2.5 \mathrm{M} n$-BuLi in THF (14.8 mL, $\left.37.1 \mathrm{mmol}\right)$ was added dropwise at $80{ }^{\circ} \mathrm{C}$. The reaction mixture was stirred additional $2 \mathrm{~h}$ at -80 to $-60{ }^{\circ} \mathrm{C}$, then cooled to $-80{ }^{\circ} \mathrm{C}$ and trimethyl borate $(5.2 \mathrm{~mL}, 46.3 \mathrm{mmol})$ was added dropwise. Then the reaction mixture was stirred overnight at room temperature, quenched with $2 \mathrm{M} \mathrm{HCl}(46 \mathrm{~mL})$ and stirred $20 \mathrm{~min}$ at room temperature. The reaction mixture was extracted with DCM $(3 \times 75 \mathrm{~mL})$, washed with brine $(50$ $\mathrm{mL}$ ), dried over anhydrous $\mathrm{Na}_{2} \mathrm{SO}_{4}$ and concentrated under reduced pressure. The solid residue was purified using column chromatography on silica gel (eluent: hexanes/ethyl acetate $=4: 1$ ) affording the desired product as a white solid in $57 \%$ yield. $\boldsymbol{R}_{\mathbf{f}} 0.25\left(\mathrm{SiO}_{2}\right.$, hexanes/EA=4:1); ${ }^{1} \mathbf{H}$ NMR $\left(600 \mathrm{MHz}, \mathrm{CDCl}_{3}\right) \delta 8.01(\mathrm{~d}, J=8.5 \mathrm{~Hz}, 2 \mathrm{H}), 7.29(\mathrm{dd}, J=8.5,7.3 \mathrm{~Hz}, 4 \mathrm{H}), 7.18-7.13$ (m, 4H), $7.11-7.06(\mathrm{~m}, 4 \mathrm{H}) .{ }^{1} \mathrm{H}$ NMR shifts match those reported for this compound in ref. [9].

\subsubsection{4-[N,N-Bis(4-methoxyphenyl)amino]phenylboronic acid}

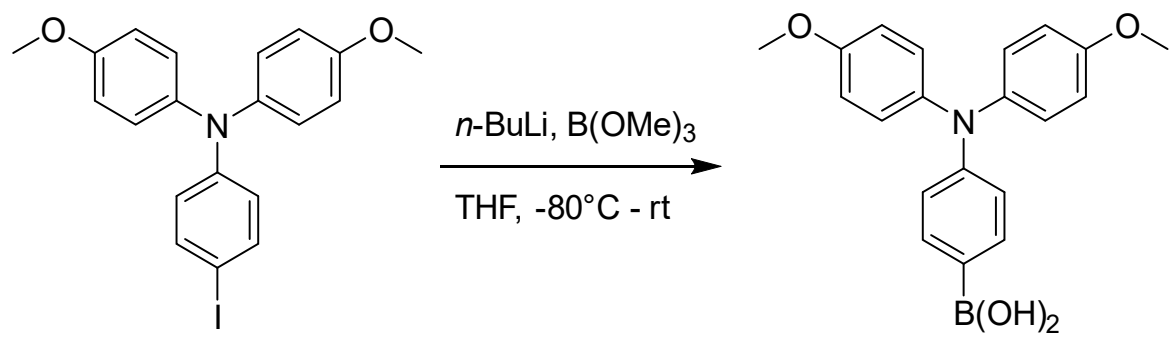

4-Iodo- $N, N$-bis(4-methoxyphenyl)aniline (4.1 g, $9.51 \mathrm{mmol}$; see section 2.3.2.2) was dissolved in dry THF $(30 \mathrm{~mL})$. The mixture was cooled to $-80{ }^{\circ} \mathrm{C}$ and then $1.6 \mathrm{M} n$-BuLi in hexanes $(8.9 \mathrm{~mL}$, $14.3 \mathrm{mmol}$ ) was added dropwise at $-80^{\circ} \mathrm{C}$. The reaction mixture was stirred additional $2 \mathrm{~h}$ at -80 to $-60^{\circ} \mathrm{C}$, then cooled to $-80^{\circ} \mathrm{C}$ and trimethyl borate $(2.1 \mathrm{~mL}, 19.0 \mathrm{mmol})$ was added dropwise. Subsequently, the reaction mixture was stirred overnight at room temperature, then quenched with $2 \mathrm{M} \mathrm{HCl}(19 \mathrm{~mL})$ and stirred for $20 \mathrm{~min}$ at room temperature. The reaction mixture was extracted with DCM $(3 \times 40 \mathrm{~mL})$, washed with brine $(30 \mathrm{~mL})$, dried over anhydrous $\mathrm{Na}_{2} \mathrm{SO}_{4}$ and 
concentrated under reduced pressure. The solid residue was purified using column chromatography on silica gel (eluent: hexanes/ethyl acetate $=3: 1$ ) affording the desired product as a white solid in $41 \%$ yield. $\boldsymbol{R}_{\mathbf{f}} 0.2\left(\mathrm{SiO}_{2}\right.$, hexanes/EA=3:1); ${ }^{1} \mathbf{H} \mathbf{~ N M R}\left(600 \mathrm{MHz}, \mathrm{CDCl}_{3}\right) \delta$ $7.94(\mathrm{~d}, J=8.6 \mathrm{~Hz}, 2 \mathrm{H}), 7.13-7.10(\mathrm{~m}, 4 \mathrm{H}), 6.93(\mathrm{~d}, J=8.6 \mathrm{~Hz}, 2 \mathrm{H}), 6.87-6.85(\mathrm{~m}, 4 \mathrm{H}), 3.81$ (s, 6H). ${ }^{1} \mathrm{H}$ NMR shifts match those reported for this compound in ref. [10]. 


\section{Synthesis of arylamine-functionalized 2-H-benzothiazoles}

\section{$3.1 \quad 6-(N, N$-Diphenylamino)benzothiazole (btz-I)}

\subsubsection{Method A - Arylation of benzothiazol-6-amine with trifenylsulfonium triflate}

\subsubsection{Synthesis of diphenyliodonium triflate}

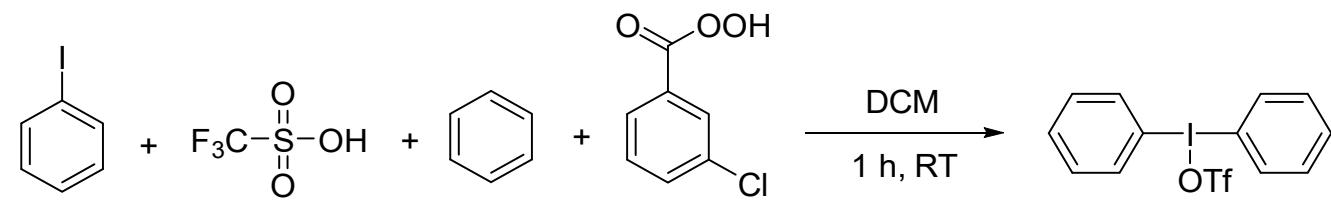

A flame-dried flask was charged with dry dichloromethane $(1 \mathrm{~L})$ under argon atmosphere and iodobenzene $(32.8 \mathrm{~mL}, 297 \mathrm{mmol})$ was added. Then, 75\% 3-chloroperoxybenzoic acid (74.46 g, $324 \mathrm{mmol}$ ) was added in several portions. After $10 \mathrm{~min}$ of stirring, benzene ( $34.1 \mathrm{~mL}, 383 \mathrm{mmol})$ was added and the mixture was cooled to $0{ }^{\circ} \mathrm{C}$. Triflic acid $(78 \mathrm{~mL}, 883 \mathrm{mmol})$ was added during $1 \mathrm{~h}$ at $0{ }^{\circ} \mathrm{C}$ and the mixture was stirred additional $1 \mathrm{~h}$ at room temperature. The mixture was poured into diethyl ether $(2 \mathrm{~L})$, cooled to $0{ }^{\circ} \mathrm{C}$ and stirred $10 \mathrm{~min}$. The precipitate was filtered off, washed with diethyl ether $(2 \times 250 \mathrm{~mL})$ and dried under reduced pressure. The product was obtained as a white crystalline solid (76.67 g, 60\%). ${ }^{1} \mathbf{H}$ NMR (600 MHz, DMSO- $\left.d_{6}\right) \delta 8.25(\mathrm{~d}, J=7.8 \mathrm{~Hz}, 4 \mathrm{H})$, $7.67(\mathrm{t}, J=7.4 \mathrm{~Hz}, 2 \mathrm{H}), 7.54(\mathrm{t}, J=7.8 \mathrm{~Hz}, 4 \mathrm{H}) .{ }^{1} \mathrm{H}$ NMR shifts match those reported for this compound in ref. [11].

\subsubsection{Synthesis of triphenylsulfonium triflate}

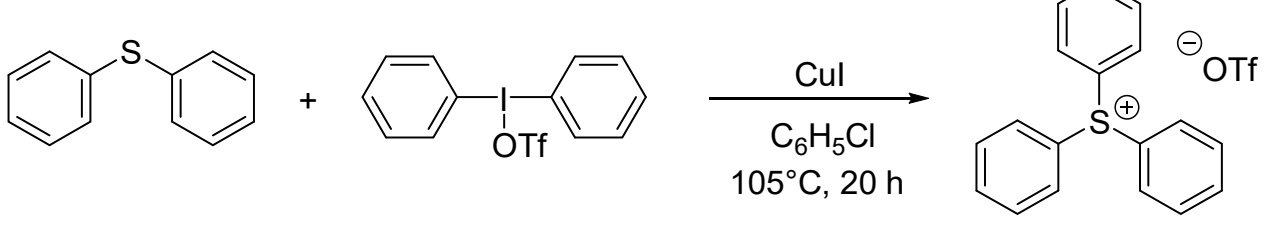

Diphenyliodonium triflate $(21.36 \mathrm{~g}, 49.7 \mathrm{mmol})$ was dissolved in dry chlorobenzene $(420 \mathrm{~mL})$ under argon atmosphere. Diphenyl sulfide (13.9 $\mathrm{mL}, 74.48 \mathrm{mmol})$ was added, followed by addition of $\mathrm{CuI}$ (472 $\mathrm{mg}, 2.48 \mathrm{mmol}, 0.05 \mathrm{eq})$. The reaction mixture was heated to $105{ }^{\circ} \mathrm{C}$ in tightly closed flask overnight. The cooled reaction mixture was poured into hexanes $(1 \mathrm{~L})$ and stirred $10 \mathrm{~min}$. The product was filtered off, washed with hexanes $(2 \times 200 \mathrm{~mL})$, dissolved in $\mathrm{CHCl}_{3}$ and filtered again. Organic solvent was removed under reduced pressure, affording triphenylsulfonium triflate $(19.1 \mathrm{~g}, 93 \%)$ as a light brown solid. ${ }^{1} \mathbf{H}$ NMR (600 MHz, DMSO-d6) $\delta$ 7.75-7.79 $(\mathrm{m}, 3 \mathrm{H}), 7.68-7.75(\mathrm{~m}, 12 \mathrm{H}) .{ }^{1} \mathrm{H}$ NMR shifts match those reported for this compound in ref. [12]. 


\subsubsection{Synthesis of 6-(N,N-diphenylamino)benzothiazole (btz-I)}

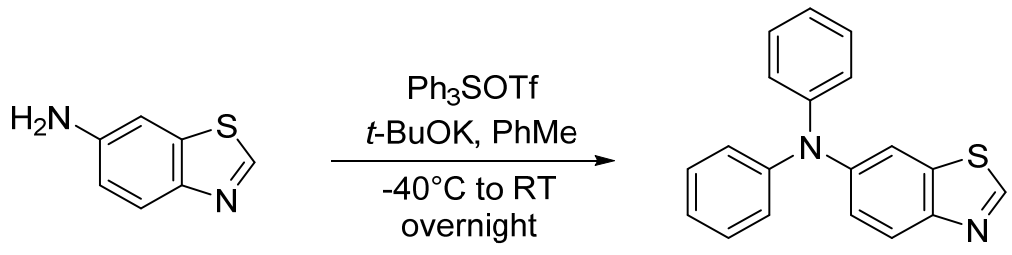

Benzothiazol-6-amine (4.8 g, $32.0 \mathrm{mmol}, 1.0 \mathrm{eq})$ was dissolved in dry toluene $(500 \mathrm{~mL})$ under argon atmosphere, cooled to $-40^{\circ} \mathrm{C}$, followed by addition of triphenylsulfonium triflate $(29.0 \mathrm{~g}$, $70.3 \mathrm{mmol}, 2.2 \mathrm{eq})$ and tert-BuOK (8.6 g, $76.7 \mathrm{mmol}, 2.4 \mathrm{eq})$. The reaction mixture was stirred for additional $2 \mathrm{~h}$ at $-40{ }^{\circ} \mathrm{C}$, then cooling bath was removed and the reaction was stirred at $\mathrm{rt}$ overnight. The reaction mixture was poured into water $(600 \mathrm{~mL})$ and extracted with ethyl acetate $(3 \times 200 \mathrm{~mL})$. Combined organic phases were washed with brine, dried with anhydrous $\mathrm{Na}_{2} \mathrm{SO}_{4}$, filtered and solvent was removed under reduced pressure. The solid residue was purified by column chromatography on silica gel (eluent: hexanes/ethyl acetate - 10:1 to 8:2) affording the desired product as a sandy-coloured solid (2.85 g, 30\%). $\boldsymbol{R}_{\mathbf{f}} 0.26\left(\mathrm{SiO}_{2}\right.$, hexanes/EA=10:1 to 8:2); Mp 159 - $160{ }^{\circ} \mathrm{C} .{ }^{1} \mathbf{H}$ NMR $\left(600 \mathrm{MHz}, \mathrm{DMSO}-d_{6}\right) \delta 9.24$ (s, 1H), 7.98 (d, J=8.8 Hz, 1H), 7.73 $(\mathrm{d}, J=2.1 \mathrm{~Hz}, 1 \mathrm{H}), 7.32(\mathrm{dd}, J=8.4,7.5 \mathrm{~Hz}, 4 \mathrm{H}), 7.18(\mathrm{dd}, J=8.8,2.1 \mathrm{~Hz}, 1 \mathrm{H}), 7.06(\mathrm{dd}, 2 \mathrm{H}, J$ = 7.5), $7.04(\mathrm{~d}, J=8.4 \mathrm{~Hz}, 6 \mathrm{H}) .{ }^{13} \mathbf{C}$ NMR (75 MHz, DMSO-d6) $\delta 155.3,149.5,147.8,145.6$, 135.5, 130.1, 124.3, 124.0, 123.7, 123.6, 117.2. Anal. Calcd for $\mathrm{C}_{19} \mathrm{H}_{14} \mathrm{~N}_{2} \mathrm{~S}: \mathrm{C}, 75.47 ; \mathrm{H}, 4.67$; N, 9.26. Found: C, 75.55; H, 4.65; N, 9.31. IR (ATR): v 2113 (w), 1582 (s), 1490 (s), 1469 (s), 1290 (s), 1273 (s), 867 (s), 841 (s), 754 (s) $\mathrm{cm}^{-1}$.

\subsubsection{Method B - Arylation of benzothiazol-6-amine with 4-fluoronitrobenzene}

\subsubsection{Synthesis of 6-[N, N-bis(4-nitrophenyl)amino]benzothiazole}

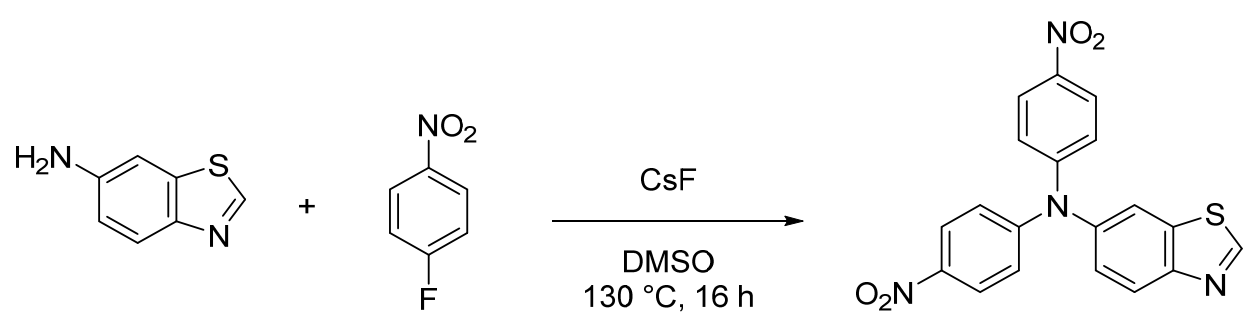

Benzothiazol-6-amine (8.95 g, $59.6 \mathrm{mmol})$ and $\mathrm{CsF}(22.63 \mathrm{~g}, 149 \mathrm{mmol})$ were placed in a dried flask, the mixture was dried under reduced pressure for $30 \mathrm{~min}$ and the flask was filled with argon. Dry DMSO $(100 \mathrm{~mL})$ was added, followed by 4-fluoronitrobenzene $(17.1 \mathrm{~mL}, 131.1 \mathrm{mmol})$ and the mixture was heated to $130{ }^{\circ} \mathrm{C}$ in an oil bath for $16 \mathrm{~h}$. After this time, the cooled reaction mixture was slowly poured into water $(1.5 \mathrm{~L})$. To remove unreacted 4-fluoronitrobenzene from 
the precipitated product, a mixture of $i$-hexane and ethyl acetate $(300 \mathrm{~mL}, 10 / 1)$ was added and after thorough stirring, organic layer was discarded. This procedure was repeated 3 more times, each time using $200 \mathrm{~mL}$ of $i$-hexane/ethyl-acetate mixture (10/1). The water layer containing solid residue was filtered and the precipitate washed with water. The obtained solid was dissolved in DCM and filtered. The crude product was precipitated by addition of hexane to filtrate. Solvents were removed under reduced pressure and the solid residue was purified by crystallization from hexanes/ethyl acetate affording the product as a bright-orange solid (19.04 g, $81 \%$ yield). Mp 217.6 - $219^{\circ} \mathrm{C} .{ }^{1} \mathbf{H}$ NMR $\left(600 \mathrm{MHz}, \mathrm{DMSO}-d_{6}\right) \delta 9.43(\mathrm{~s}, 1 \mathrm{H}), 8.18(\mathrm{~d}, J=9.2 \mathrm{~Hz}, 4 \mathrm{H}), 8.15(\mathrm{~d}$, $J=2.1 \mathrm{~Hz}, 1 \mathrm{H}), 7.41(\mathrm{dd}, J=8.7,2.2 \mathrm{~Hz}, 1 \mathrm{H}), 7.24(\mathrm{~d}, J=9.2 \mathrm{~Hz}, 4 \mathrm{H}) .{ }^{13} \mathbf{C}$ NMR $(151 \mathrm{MHz}$, DMSO- $\left.d_{6}\right) \delta 158.1,152.1,152.1,142.6,142.3,136.0,126.5,126.1,125.1,123.1,122.1$. Anal.

Calcd for $\mathrm{C}_{19} \mathrm{H}_{12} \mathrm{~N}_{4} \mathrm{O}_{4} \mathrm{~S}$ : C, 58.16; H, 3.08; N, 14.28. Found: C, 58.40; H, 3.20; N, 14.12. IR (ATR): $v 3077$ (w), 1730 (w), 1578 (s), 1491 (s), 1295 (s), 1278 (s), 1106 (s), 842 (s) cm cm $^{-1}$.

\subsubsection{Synthesis of 6-[N, N-bis(4-aminophenyl)amino]benzothiazole}

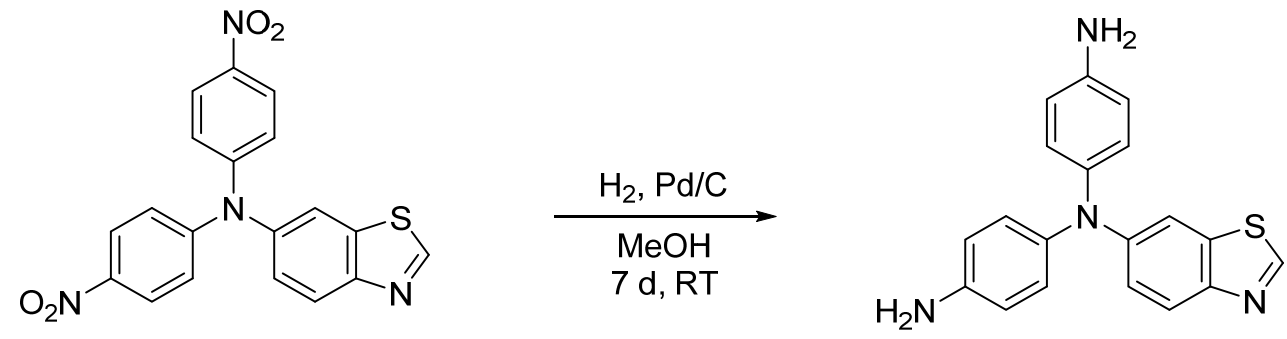

6-[N,N-bis(4-nitrophenyl)amino]benzothiazole (8.0 g, $20.4 \mathrm{mmol})$ was suspended in $\mathrm{MeOH}$ (350 $\mathrm{mL})$ under argon atmosphere and $10 \%$ palladium on carbon $(2.17 \mathrm{~g}, 2.04 \mathrm{mmol})$ was added. Argon atmosphere was removed by reduced pressure and exchanged for $\mathrm{H}_{2}$ (this exchange was repeated 3 times). The reaction mixture was stirred at room temperature under $\mathrm{H}_{2}$ atmosphere for 7 days, then filtered through Celite and washed with $\mathrm{MeOH}$. Methanol was removed under reduced pressure, the solid residue dissolved in DCM and filtered to get rid of inorganic solids. DCM from filtrate was removed under reduced pressure and the product was isolated as a brown powder (6.53 g, $99 \%$ yield). Mp $165-166.8^{\circ} \mathrm{C} .{ }^{1} \mathbf{H}$ NMR (600 MHz, DMSO-d $) \delta 8.96$ (s, 1H), $7.73(\mathrm{~d}, J=9.0 \mathrm{~Hz}, 1 \mathrm{H}), 7.10(\mathrm{~d}, J=2.3 \mathrm{~Hz}, 1 \mathrm{H}), 6.83$ (d, $J=8.6 \mathrm{~Hz}, 4 \mathrm{H}), 6.54$ (d, $J=8.6 \mathrm{~Hz}$, 4H), 5.00 (s, 4H). ${ }^{13}$ C NMR (151 MHz, DMSO-d6) $\delta 151.8,148.6,146.3,146.3,136.3,135.4$, 127.8, 123.1, 117.7, 115.3, 108.4. Anal. Calcd for $\mathrm{C}_{19} \mathrm{H}_{16} \mathrm{~N}_{4} \mathrm{~S}: \mathrm{C}, 68.65 ; \mathrm{H}, 4.85 ; \mathrm{N}, 16.85$. Found: C, 68.69; H, 4.85; N, 16.93. IR (ATR): v 3329 (w), 3207 (w), 1595 (m), 1501 (s), 1464 (s), 1260 (s), $822(\mathrm{~s}) \mathrm{cm}^{-1}$. 


\subsubsection{Hydrodeamination of 6-[N, N-bis(4-aminophenyl)amino]benzothiazole}<smiles>Nc1ccc(N(c2ccc(N)cc2)c2ccc3ncsc3c2)cc1</smiles>
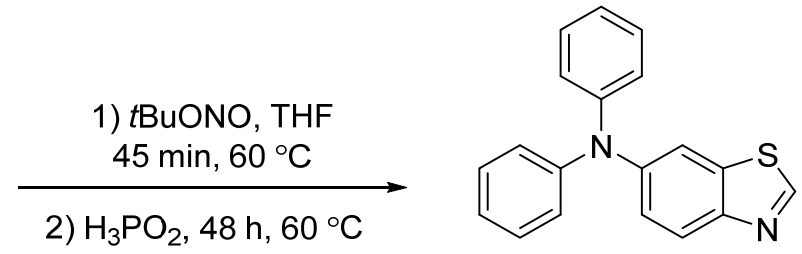

To a stirred solution of tert-butyl nitrite $(2.74 \mathrm{~mL}, 20.70 \mathrm{mmol})$ in dry THF $(45 \mathrm{~mL})$ at $60{ }^{\circ} \mathrm{C}$ was added dropwise (over the course of $30 \mathrm{~min})$ a solution of benzothiazol-6-amine $(3.277 \mathrm{~g}, 9.86$ $\mathrm{mmol})$ in dry THF $(45 \mathrm{~mL})$. The reaction mixture was stirred $45 \mathrm{~min}$ at $60{ }^{\circ} \mathrm{C}$, then $50 \%$ aq. solution of $\mathrm{H}_{3} \mathrm{PO}_{2}(20.5 \mathrm{ml}, 197 \mathrm{mmol})$ was added dropwise and the mixture was stirred for additional $24 \mathrm{~h}$ at $60{ }^{\circ} \mathrm{C}$. After cooling to rt, the reaction mixture was poured to a mixture of water $(300 \mathrm{~mL})$ and brine $(100 \mathrm{~mL})$, basified with $1 \mathrm{M} \mathrm{NaOH}(300 \mathrm{~mL})$ and extracted with ethyl acetate $(3 \times 200 \mathrm{~mL})$. Combined organic phases were washed with brine, dried over $\mathrm{Na}_{2} \mathrm{SO}_{4}$, filtered and concentrated under reduced pressure. The crude solid was purified by column chromatography on silica gel (eluent: hexanes/ethyl acetate - 10:1 to 8:2) affording the desired product as a sandycoloured solid (1.18 g, 39\%). $\boldsymbol{R}_{\mathbf{f}} 0.25\left(\mathrm{SiO}_{2}\right.$, hexanes/EA=10:1 to 8:2); Mp 159- $160{ }^{\circ} \mathrm{C} .{ }^{\mathbf{1}} \mathbf{H} \mathbf{~ N M R}$ $\left(600 \mathrm{MHz}, \mathrm{DMSO}-d_{6}\right) \delta 9.24(\mathrm{~s}, 1 \mathrm{H}), 7.98(\mathrm{~d}, J=8.8 \mathrm{~Hz}, 1 \mathrm{H}), 7.73(\mathrm{~d}, J=2.1 \mathrm{~Hz}, 1 \mathrm{H}), 7.32$ (dd, $J=8.4,7.5 \mathrm{~Hz}, 4 \mathrm{H}), 7.18(\mathrm{dd}, J=8.8,2.1 \mathrm{~Hz}, 1 \mathrm{H}), 7.06(\mathrm{dd}, 2 \mathrm{H}, J=7.5), 7.04(\mathrm{~d}, J=8.4 \mathrm{~Hz}$, 6H). ${ }^{13}$ C NMR (75 MHz, DMSO-d6) $\delta$ 155.3, 149.5, 147.8, 145.6, 135.5, 130.1, 124.3, 124.0, 123.7, 123.6, 117.2. Anal. Calcd for $\mathrm{C}_{19} \mathrm{H}_{14} \mathrm{~N}_{2} \mathrm{~S}$ : C, 75.47; H, 4.67; N, 9.26. Found: C, 75.55; H, 4.65; N, 9.31. IR (ATR): v 2113 (w), 1582 (s), 1490 (s), 1469 (s), 1290 (s), 1273 (s), 867 (s), 841 (s), $754(\mathrm{~s}) \mathrm{cm}^{-1}$. 


\subsection{Buchwald-Hartwig cross-coupling reactions}

\subsubsection{Benzothiazol-6-amine with bromobenzene and $\mathrm{Cs}_{2} \mathrm{CO}_{3}$ as a base}
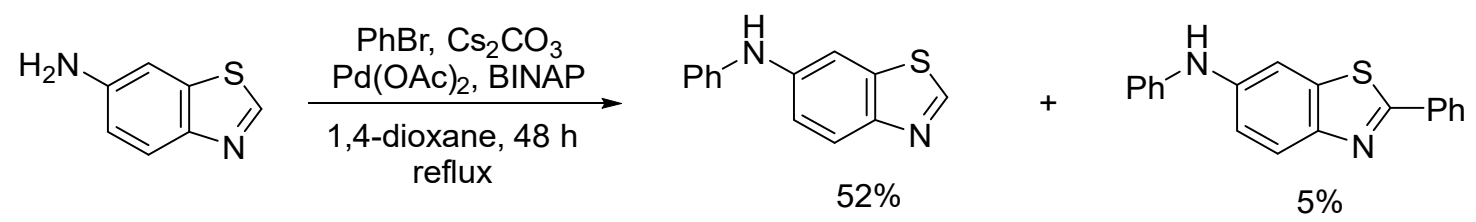

A flame-dried flask was charged with benzothiazol-6-amine (1.0 g, $3.9 \mathrm{mmol})$, BINAP (122 mg, $0.2 \mathrm{mmol}), \mathrm{Pd}(\mathrm{OAc})_{2}(35 \mathrm{mg}, 0.2 \mathrm{mmol})$ and $\mathrm{Cs}_{2} \mathrm{CO}_{3}(3.812 \mathrm{~g}, 11.7 \mathrm{mmol})$ under argon and dry 1,4-dioxane $(30 \mathrm{~mL})$ was added. Subsequently, bromobenzene $(1.0 \mathrm{~mL}, 9.8 \mathrm{mmol})$ was added under stirring and the reaction mixture was heated to reflux over $48 \mathrm{~h}$. The cooled reaction mixture was poured into water and extracted with dichloromethane $(3 \times 50 \mathrm{~mL})$. Combined organic phases were washed with brine, dried over anhydrous $\mathrm{Na}_{2} \mathrm{SO}_{4}$, filtered and concentrated under reduced pressure. The solid residue was purified using column chromatography on silica gel (eluent: hexanes/ethyl acetate $=4: 1)$ affording $N$-phenyl-benzothiazol-6-amine $(0.46 \mathrm{~g}, 52 \%)$ as a lightyellow solid as the major product. $\boldsymbol{R}_{\mathbf{f}} 0.32\left(\mathrm{SiO}_{2}\right.$, hexanes/EA=4:1); ${ }^{1} \mathbf{H} \mathbf{N M R}\left(600 \mathrm{MHz}, \mathrm{CDCl}_{3}\right)$ $\delta 8.79(\mathrm{~s}, 1 \mathrm{H}), 7.99(\mathrm{~d}, J=8.8 \mathrm{~Hz}, 1 \mathrm{H}), 7.61(\mathrm{~d}, J=2.2 \mathrm{~Hz}, 1 \mathrm{H}), 7.29-7.33(\mathrm{~m}, 2 \mathrm{H}), 7.20(\mathrm{dd}, J$ $=8.8,2.2 \mathrm{~Hz}, 1 \mathrm{H}), 7.12-7.15(\mathrm{~m}, 2 \mathrm{H}), 6.98-7.02(\mathrm{~m}, 1 \mathrm{H}), 5.88(\mathrm{~s}, 1 \mathrm{H}) .{ }^{1} \mathrm{H}$ NMR shifts match those reported for this compound in ref. [13]. Besides we isolated trace amounts of N,2-diphenylbenzothiazol-6-amine as the minor product (see also the procedure below).

\subsubsection{Benzothiazol-6-amine with bromobenzene and $t \mathrm{BuOK}$ as a base}

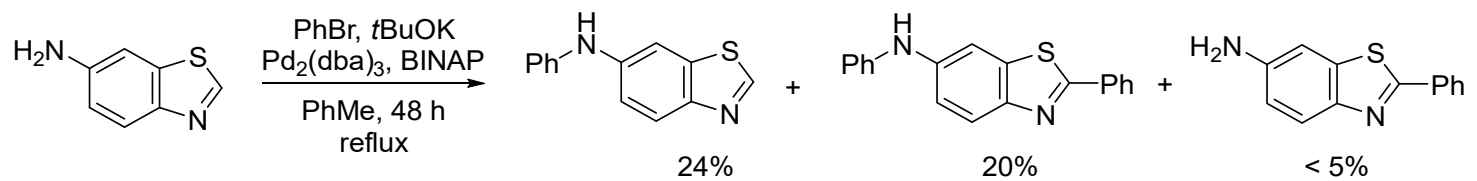

A flame-dried flask was charged with benzothiazol-6-amine $(0.5 \mathrm{~g}, 1.95 \mathrm{mmol})$, BINAP (60.8 $\mathrm{mg}, 0.10 \mathrm{mmol}), \mathrm{Pd}_{2}(\mathrm{dba})_{3}(71.5 \mathrm{mg}, 0.08 \mathrm{mmol})$ and potassium tert-butanolate $(0.66 \mathrm{~g}, 5.86$ $\mathrm{mmol})$ under argon atmosphere and dry toluene $(20 \mathrm{~mL})$ was added. Bromobenzene $(0.51 \mathrm{~mL}$, $4.88 \mathrm{mmol}$ ) was added under stirring and the mixture was heated to reflux over $48 \mathrm{~h}$. The cooled reaction mixture was poured into water and extracted with dichloromethane $(3 \times 20 \mathrm{~mL})$. Combined organic phases were washed with brine $(20 \mathrm{~mL})$, dried over anhydrous $\mathrm{Na}_{2} \mathrm{SO}_{4}$, filtered and concentrated under reduced pressure. The solid residue was purified / separated using column chromatography on silica gel (eluent: hexanes/ethyl acetate $=4: 1$ ) 


\section{$N$-Phenylbenzothiazol-6-amine}

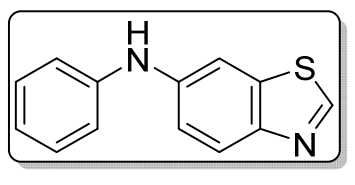

(103 mg, $24 \%$, light yellow solid) ${ }^{1} \mathbf{H}$ NMR (600 MHz, $\left.\mathrm{CDCl}_{3}\right) \delta 8.79$ (s, 1H), $7.99(\mathrm{~d}, J=8.8 \mathrm{~Hz}, 1 \mathrm{H}), 7.61(\mathrm{~d}, J=2.2 \mathrm{~Hz}, 1 \mathrm{H}), 7.29-7.33(\mathrm{~m}$, 2H), $7.20(\mathrm{dd}, J=8.8,2.2 \mathrm{~Hz}, 1 \mathrm{H}), 7.12-7.15(\mathrm{~m}, 2 \mathrm{H}), 6.98-7.02(\mathrm{~m}$,

1H), $5.88(\mathrm{~s}, 1 \mathrm{H}) .{ }^{1} \mathrm{H}$ NMR shifts match those reported for this compound in ref. [13].

\section{$N, 2-D i p h e n y l b e n z o t h i a z o l-6-a m i n e$}

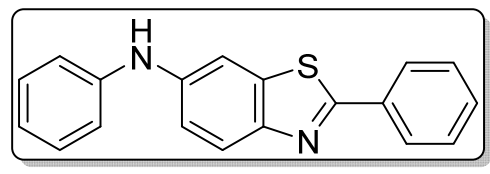

(118 mg, $20 \%$, light yellow solid) ${ }^{\mathbf{1}} \mathbf{H}$ NMR (600 MHz, $\left.\mathrm{CDCl}_{3}\right)$ $\delta 8.04(\mathrm{dd}, J=7.9,1.6 \mathrm{~Hz}, 2 \mathrm{H}), 7.94(\mathrm{~d}, J=8.7 \mathrm{~Hz}, 1 \mathrm{H}), 7.57$ $(\mathrm{d}, J=2.2 \mathrm{~Hz}, 1 \mathrm{H}), 7.47(\mathrm{~m}, 3 \mathrm{H}), 7.32(\mathrm{dd}, J=8.4,7.5 \mathrm{~Hz}, 2 \mathrm{H})$, $7.17(\mathrm{dd}, J=8.7,2.2 \mathrm{~Hz}, 1 \mathrm{H}), 7.14(\mathrm{dd}, J=8.5,0.9 \mathrm{~Hz}, 2 \mathrm{H}), 7.00(\mathrm{dd}, J=7.5,0.9 \mathrm{~Hz}, 1 \mathrm{H}), 5.87$ (br s, 1H). ${ }^{1} \mathrm{H}$ NMR shifts match those reported for this compound in ref. [14].

\section{2-Phenylbenzothiazol-6-amine}

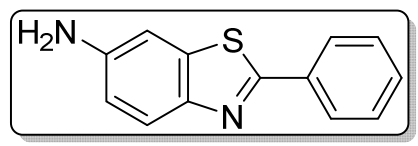

(traces, yellow oil) ${ }^{1} \mathbf{H}$ NMR $\left(600 \mathrm{MHz}, \mathrm{CDCl}_{3}\right) \delta 8.00-8.03(\mathrm{~m}, 2 \mathrm{H})$, $7.84(\mathrm{~d}, J=8.6 \mathrm{~Hz}, 1 \mathrm{H}), 7.42-7.48(\mathrm{~m}, 3 \mathrm{H}), 7.14(\mathrm{~d}, J=2.3 \mathrm{~Hz}, 1 \mathrm{H})$,

$6.85(\mathrm{dd}, J=8.6,2.3 \mathrm{~Hz}, 1 \mathrm{H}), 3.85$ (br s, 2H). 


\subsection{6-[4-( $N, N$-Diphenylamino)phenyl]benzothiazole (btz-II)}

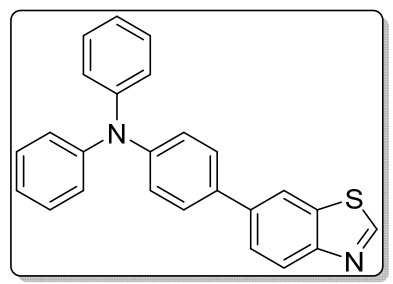

A mixture of 6-iodobenzothiazole $(1.0 \mathrm{~g}, 3.8 \mathrm{mmol}), 4-(N, N-$ diphenylamino)phenylboronic acid (1.32 g, $4.56 \mathrm{mmol}), \mathrm{Pd}\left(\mathrm{PPh}_{3}\right)_{4}$ $(0.88 \mathrm{~g}, 0.76 \mathrm{mmol})$ was dissolved in toluene $(30 \mathrm{~mL})$, ethanol $(10$ $\mathrm{mL})$ and potassium carbonate $(1.6 \mathrm{~g}, 11.4 \mathrm{mmol})$ dissolved in water (1 $\mathrm{mL}$ ) was added under argon atmosphere. The reaction mixture was heated to reflux for 12 hours. After cooling, the reaction mixture was concentrated under reduced pressure and purified using column chromatography on silica gel (eluent: hexanes/ethyl acetate $=$ 4:1) to give btz-II as a yellow solid (1.05 g, $73 \%$ yield). $\boldsymbol{R}_{\mathbf{f}} 0.2\left(\mathrm{SiO}_{2}\right.$, hexanes/EA=4:1); $\mathbf{M p} 173$ $-174{ }^{\circ} \mathrm{C} .{ }^{1} \mathbf{H}$ NMR $\left(600 \mathrm{MHz}, \mathrm{CDCl}_{3}\right) \delta 8.97(\mathrm{~s}, 1 \mathrm{H}), 8.16(\mathrm{~d}, J=8.4 \mathrm{~Hz}, 1 \mathrm{H}), 8.12(\mathrm{~d}, J=1.8$ $\mathrm{Hz}, 1 \mathrm{H}), 7.73(\mathrm{dd}, J=8.5,1.8 \mathrm{~Hz}, 1 \mathrm{H}), 7.53(\mathrm{~d}, J=8.6 \mathrm{~Hz}, 2 \mathrm{H}), 7.28$ (t, $J=7.9 \mathrm{~Hz}, 4 \mathrm{H}), 7.16$ (t, $J=8.7 \mathrm{~Hz}, 6 \mathrm{H}), 7.05(\mathrm{t}, J=7.3 \mathrm{~Hz}, 2 \mathrm{H}) .{ }^{13} \mathbf{C} \mathbf{N M R}\left(151 \mathrm{MHz}, \mathrm{CDCl}_{3}\right) \delta 153.7,152.2,147.5$, $138.6,134.5,134.1,129.3,128.1,125.5,124.6,123.7,123.6,123.1,119.5$. Anal. Calcd for $\mathrm{C}_{25} \mathrm{H}_{18} \mathrm{~N}_{2} \mathrm{~S}: \mathrm{C}, 79.33 ; \mathrm{H}, 4.79 ; \mathrm{N}, 7.40$. Found: C, 79.44; H, 4.71; N, 7.29. IR (ATR): v 2959 (w), 2921 (w), 1584 (s), 1509 (s), 1486 (s), 1259 (s), 813 (s) $\mathrm{cm}^{-1}$.

\section{$3.46-\{4-[N, N$-Bis(4-methoxyphenyl)amino]phenyl\}benzothiazole (btz-III)}

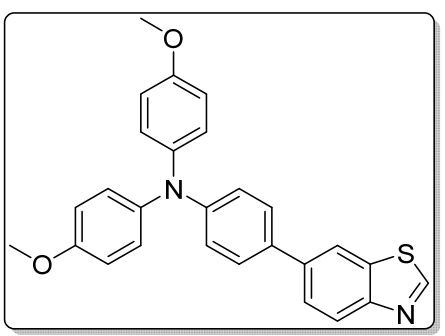

A dried flask was charged with 6-iodobenzothiazole $(0.91 \mathrm{~g}, 3.5$ mmol), 4-[N,N-bis(4-methoxyphenyl)amino]phenylboronic acid (1.29 g, $3.68 \mathrm{mmol})$ and $\mathrm{Pd}\left(\mathrm{PPh}_{3}\right)_{4}(0.81 \mathrm{~g}, 0.7 \mathrm{mmol})$, and then flushed with argon. Degassed solution of $\mathrm{K}_{2} \mathrm{CO}_{3}(1.453 \mathrm{~g}, 10.51$ $\mathrm{mmol})$ in water $(5.3 \mathrm{~mL})$ was added, followed by addition of a

degassed mixture of toluene $(20 \mathrm{~mL})$ and $\mathrm{EtOH}(8 \mathrm{~mL})$. The reaction mixture was heated to reflux for $30 \mathrm{~h}$. The reaction mixture was diluted with water and extracted with dichloromethane $(3 \times 15$ $\mathrm{mL}$ ). Combined organic phases were washed with brine, dried over anhydrous $\mathrm{Na}_{2} \mathrm{SO}_{4}$, filtered and concentrated under reduced pressure. The solid residue was purified by column chromatography on silica gel (eluent: hexanes / ethyl acetate $=10: 1)$ affording btz-III as a yellow solid (0.91 g, $60 \%$ yield). $\boldsymbol{R}_{\mathbf{f}} 0.2\left(\mathrm{SiO}_{2}\right.$, hexanes/EA=10:1); Mp $55-57{ }^{\circ} \mathrm{C} .{ }^{1} \mathbf{H} \mathbf{~ N M R}(600 \mathrm{MHz}$, $\left.\mathrm{CDCl}_{3}\right) \delta 8.95(\mathrm{~s}, 1 \mathrm{H}), 8.14(\mathrm{~d}, J=8.6 \mathrm{~Hz}, 1 \mathrm{H}), 8.09(\mathrm{~d}, J=1.9 \mathrm{~Hz}, 1 \mathrm{H}), 7.71(\mathrm{dd}, J=8.5,1.9$ $\mathrm{Hz}, 1 \mathrm{H}), 7.46(\mathrm{~d}, J=8.7 \mathrm{~Hz}, 2 \mathrm{H}), 7.10(\mathrm{~d}, J=9.0 \mathrm{~Hz}, 4 \mathrm{H}), 7.01(\mathrm{~d}, J=8.7 \mathrm{~Hz}, 2 \mathrm{H}), 6.85(\mathrm{~d}, J=$ $9.0 \mathrm{~Hz}, 4 \mathrm{H}), 3.80$ (s, 6H). ${ }^{13} \mathbf{C}$ NMR $\left(151 \mathrm{MHz}, \mathrm{CDCl}_{3}\right) \delta 156.2,153.7,152.2,148.7,140.9,139.0$, $134.7,132.2,128.0,126.9,125.6,123.7,120.7,119.4,115.0,55.7$, 55.7. Anal. Calcd for $\mathrm{C}_{27} \mathrm{H}_{22} \mathrm{~N}_{2} \mathrm{O}_{2} \mathrm{~S}$ : C, 73.95; H, 5.06; N, 6.39. Found: C, 74.09; H, 5.06; N, 6.37. IR (ATR): v 2996 (w), $2831(\mathrm{w}), 1500(\mathrm{~s}), 1462(\mathrm{~s}), 1236(\mathrm{~s}), 1032(\mathrm{~s}), 825(\mathrm{~s}) \mathrm{cm}^{-1}$. 


\subsection{6-[4-( $N, N$-Diphenylamino)phenylethynyl]benzothiazole (btz-IV)}

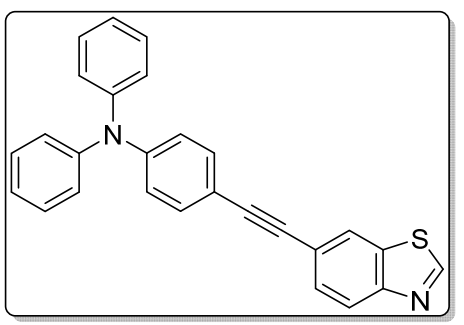

6-Iodobenzothiazole (1.0 g, $3.88 \mathrm{mmol}), \mathrm{Pd}\left(\mathrm{PPh}_{3}\right)_{2} \mathrm{Cl}_{2}(403 \mathrm{mg}$, $0.575 \mathrm{mmol})$ and $\mathrm{CuI}(55 \mathrm{mg}, 0.287 \mathrm{mmol})$ were dissolved under argon atmosphere in a mixture of dry THF $(14 \mathrm{~mL})$ and dry $\mathrm{Et}_{3} \mathrm{~N}$ $(8.6 \mathrm{~mL})$. The mixture was stirred $15 \mathrm{~min}$ at room temperature, then 4-(N,N-diphenylamino)phenylacetylene (1.149 g, $4.268 \mathrm{mmol})$ in

THF $(8 \mathrm{~mL})$ was added dropwise and the reaction mixture under inert atmosphere was stirred overnight at room temperature. Then the reaction mixture was concentrated under reduced pressure and the solid residue was purified by column chromatography on silica gel (eluent: hexanes/ethyl acetate $=9: 1)$ providing btz-IV as a white solid $(1.16 \mathrm{~g}, 75 \%) . \boldsymbol{R}_{\mathbf{f}} 0.22\left(\mathrm{SiO}_{2}\right.$, hexanes/EA=9:1); Mp $184-186{ }^{\circ} \mathrm{C} .{ }^{1} \mathbf{H}$ NMR (600 MHz, $\left.\mathrm{CDCl}_{3}\right) \delta 9.01$ (s, 1H), $8.11(\mathrm{~d}, J=1.6$ $\mathrm{Hz}, 1 \mathrm{H}), 8.08$ (d, $J=8.5 \mathrm{~Hz}, 1 \mathrm{H}), 7.64(\mathrm{dd}, J=8.5,1.6 \mathrm{~Hz}, 1 \mathrm{H}), 7.39$ (d, $J=8.7 \mathrm{~Hz}, 2 \mathrm{H}), 7.28$ $(\mathrm{dd}, J=8.5,7.4 \mathrm{~Hz}, 4 \mathrm{H}), 7.12(\mathrm{dd}, J=8.5,1.1 \mathrm{~Hz}, 4 \mathrm{H}), 7.07(\mathrm{dd}, J=7.4,1.1 \mathrm{~Hz}, 2 \mathrm{H}), 7.02(\mathrm{~d}, J$ $=8.7 \mathrm{~Hz}, 2 \mathrm{H}) .{ }^{13} \mathbf{C}$ NMR $\left(151 \mathrm{MHz}, \mathrm{CDCl}_{3}\right) \delta 154.9,152.8,148.3,147.3,134.1,132.8,129.9$, 129.6, 125.3, 125.0, 123.8, 123.6, 122.4, 121.4, 115.8, 91.0, 88.3. Anal. Calcd for $\mathrm{C}_{27} \mathrm{H}_{18} \mathrm{~N}_{2} \mathrm{~S}$ : C, 80.57; H, 4.51; N, 6.96. Found: C, 80.50; H, 4.43; N, 6.90. IR (ATR): v 2875 (w), 2362 (w), 1684 (s), 1477 (s), 1437 (s), 1196 (s), 1106 (s), 1067 (s) $\mathrm{cm}^{-1}$.

\subsection{6- $\{4-[N, N$-Bis(4-methoxyphenyl)amino]phenylethynyl\}benzothiazole (btz-V)}

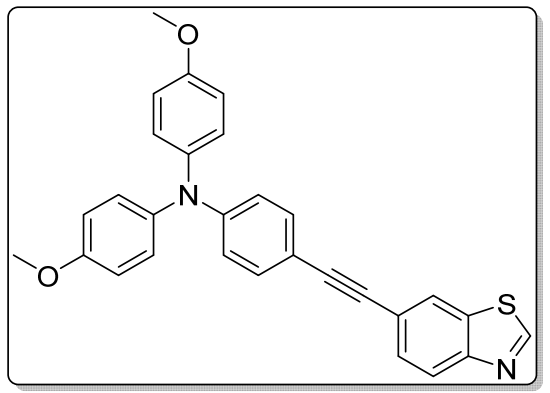

6-Iodobenzothiazole $(0.50 \mathrm{~g}, 1.92 \mathrm{mmol}), \mathrm{Pd}\left(\mathrm{PPh}_{3}\right)_{2} \mathrm{Cl}_{2}(80$ $\mathrm{mg}, 0.115 \mathrm{mmol})$ and $\mathrm{CuI}(7.3 \mathrm{mg}, 0.038 \mathrm{mmol})$ were dissolved under argon atmosphere in dry THF $(10 \mathrm{~mL})$ and dry $\mathrm{Et}_{3} \mathrm{~N}(2.7 \mathrm{~mL})$. The reaction mixture was stirred $15 \mathrm{~min}$ at room temperature, then 4-[N,N-bis(4-methoxyphenyl)amino]phenylacetylene (742 mg, $1.92 \mathrm{mmol})$ in THF (4 mL)

was added dropwise and the mixture under inert atmosphere was stirred overnight at room temperature. Then the reaction mixture was concentrated under reduced pressure and the solid residue was purified using column chromatography on silica gel (eluent: hexanes/ethyl acetate $=$ 9:1) affording btz-V as a yellow solid (0.63 g, $71 \%$ yield). $\boldsymbol{R}_{\mathbf{f}} 0.15\left(\mathrm{SiO}_{2}\right.$, hexanes/EA=9:1); Mp $59-61{ }^{\circ} \mathrm{C} .{ }^{1} \mathbf{H}$ NMR $\left(600 \mathrm{MHz}, \mathrm{CDCl}_{3}\right) \delta 9.00(\mathrm{~s}, 1 \mathrm{H}), 8.10(\mathrm{~d}, J=0.9 \mathrm{~Hz}, 1 \mathrm{H}), 8.07(\mathrm{~d}, J=8.5$ Hz, 1H), 7.63 (dd, $J=8.5,1.4 \mathrm{~Hz}, 1 \mathrm{H}), 7.33$ (d, $J=8.7 \mathrm{~Hz}, 2 \mathrm{H}), 7.08$ (d, $J=8.9 \mathrm{~Hz}, 4 \mathrm{H}), 6.86$ $(\mathrm{dd}, J=8.9,2.5 \mathrm{~Hz}, 6 \mathrm{H}), 3.81$ (s, 6H). ${ }^{13} \mathbf{C} \mathbf{N M R}\left(151 \mathrm{MHz}, \mathrm{CDCl}_{3}\right) \delta 156.4,154.6,149.0,140.1$, $132.5,129.6,127.2,124.7,123.4,121.5,119.5,114.8,113.4,91.2,87.5$, 55.5. Anal. Calcd for 
$\mathrm{C}_{29} \mathrm{H}_{22} \mathrm{~N}_{2} \mathrm{O}_{2} \mathrm{~S}$ : C, 75.30; H, 4.79; N, 6.06. Found: C, 75.35; H, 4.83; N, 6.16. IR (ATR): v 1587 (s), 1499 (s), 1320 (s), 1285 (s), 1236 (s), 1030 (s), 824 (s) cm $\mathrm{cm}^{-1}$.

\section{7 (E)-6-[4-(N,N-Diphenylamino)phenylethenyl]benzothiazole (btz-VI)}

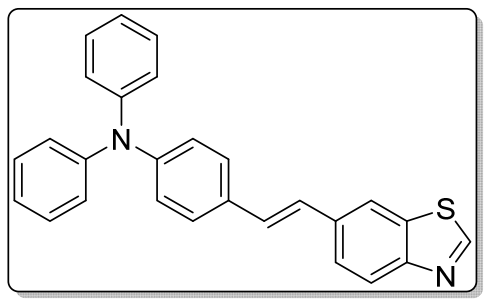

To a solution of 4-(N,N-diphenylamino)phenylacetylene $(0.7 \mathrm{~g}$, $2.6 \mathrm{mmol})$ in THF $(6 \mathrm{~mL})$ was added catecholborane $(0.33 \mathrm{~mL}$, $3.12 \mathrm{mmol}$ ) under an atmosphere of nitrogen and the solution was heated to reflux. After $1.5 \mathrm{~h}$ an additional amount of catecholborane $(0.28 \mathrm{~mL}, 2.6 \mathrm{mmol})$ was added and heating was

continued for further $2 \mathrm{~h}$. After cooling to $\mathrm{rt}$, a solution of 6-iodobenzothiazole $(0.564 \mathrm{~g}, 2.16$ $\mathrm{mmol})$ and $\mathrm{Pd}\left(\mathrm{PPh}_{3}\right)_{4}(0.499 \mathrm{mg}, 0.432 \mathrm{mmol})$ in THF $(6 \mathrm{~mL})$ was added dropwise, and the reaction mixture was stirred at room temperature for $30 \mathrm{~min}$. Consequently, $20 \%$ aqueous solution of $\mathrm{Na}_{2} \mathrm{CO}_{3}(2.6 \mathrm{~mL})$ was added slowly and the mixture was heated to reflux for $20 \mathrm{~h}$. After this time, the reaction mixture was cooled, quenched with water $(20 \mathrm{~mL})$, and extracted with ethyl acetate $(3 \times 30 \mathrm{~mL})$. The combined organic extracts were washed with water, brine and dried over anhydrous $\mathrm{Na}_{2} \mathrm{SO}_{4}$. Organic solvents were evaporated under reduced pressure and the solid residue was purified by column chromatography on silica gel (eluent: ethyl acetate/hexanes =1:9) affording btz-VI as a yellow solid ( $0.52 \mathrm{~g}, 60 \%$ yield). $\boldsymbol{R}_{\mathbf{f}} 0.2\left(\mathrm{SiO}_{2}, \mathrm{EA} / \mathrm{hexanes}=1: 9\right)$; $\mathbf{M p} 59-$ $60{ }^{\circ} \mathrm{C} .{ }^{1} \mathrm{H}$ NMR $\left(600 \mathrm{MHz}, \mathrm{CDCl}_{3}\right) \delta 8.96(\mathrm{~s}, 1 \mathrm{H}), 8.10(\mathrm{~d}, J=8.5 \mathrm{~Hz}, 1 \mathrm{H}), 8.04(\mathrm{~d}, J=1.8 \mathrm{~Hz}$, 1H), $7.70(\mathrm{dd}, J=8.5,1.8 \mathrm{~Hz}, 1 \mathrm{H}), 7.42(\mathrm{~d}, J=8.7 \mathrm{~Hz}, 2 \mathrm{H}), 7.29$ (d, $J=7.4 \mathrm{~Hz}, 2 \mathrm{H}), 7.19-7.03$ $(\mathrm{m}, 12 \mathrm{H}) .{ }^{13} \mathbf{C} \mathbf{N M R}\left(151 \mathrm{MHz}, \mathrm{CDCl}_{3}\right) \delta 153.7,152.6,147.7,147.5,135.6,134.6,131.0,129.3$, 129.3, 127.4, 126.1, 124.6, 124.6, 123.6, 123.4, 123.2, 119.4. Anal. Calcd for $\mathrm{C}_{27} \mathrm{H}_{20} \mathrm{~N}_{2} \mathrm{~S}$ : C, 80.17; H, 4.98; N, 6.93. Found: C, 80.35; H, 5.10; N, 7.23. IR (ATR): v 3021 (w), 2922 (w), 1585 (s), 1506 (s), 1487 (s), 1324 (s), 1273 (s), 752 (s) $\mathrm{cm}^{-1}$. 


\section{Oxidative homocoupling of 2-H-benzothiazoles}

\subsection{Synthesis of $2,2^{\prime}$-bibenzothiazole}<smiles>c1ccc2scnc2c1</smiles>
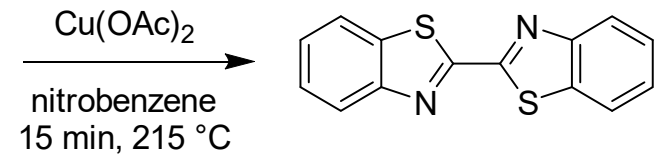

Method A: Benzothiazole $(8.07 \mathrm{~mL}, 0.074 \mathrm{~mol})$ was dissolved in nitrobenzene $(20 \mathrm{~mL})$ and the mixture was heated to $215^{\circ} \mathrm{C}$. $\mathrm{Cu}(\mathrm{OAc})_{2}(13.44 \mathrm{~g}, 0.074 \mathrm{~mol})$ - dried prior to the synthesis under reduced pressure at $155^{\circ} \mathrm{C}$ for 3 hours - was added portionwise over the course of 12 min and after complete addition the reaction mixture was heated for another $3 \mathrm{~min}$. Subsequently, the mixture was cooled to room temperature, filtered and the slurry washed with hot saturated solution od EDTA in water $(300 \mathrm{~mL})$ and then with $93 \%$ ethanol $(100 \mathrm{~mL})$ to give 2,2 '-bibenzothiazole as a gold-yellow solid $(7.4 \mathrm{~g})$ in $75 \%$ yield.<smiles>c1ccc2scnc2c1</smiles>

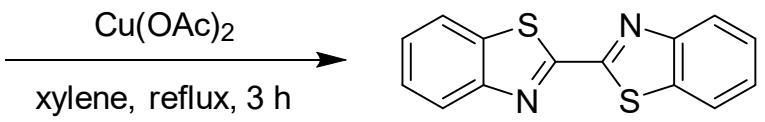

Method B: Benzothiazole $(8.07 \mathrm{~mL}, 0.074 \mathrm{~mol})$ was dissolved in a mixture of xylenes $(50 \mathrm{~mL})$ and the mixture was heated to reflux. Then $\mathrm{Cu}(\mathrm{OAc})_{2}(13.44 \mathrm{~g}, 0.074 \mathrm{mmol})-$ dried prior to the synthesis under reduced pressure at $155^{\circ} \mathrm{C}$ for 3 hours - was added portionwise and the reaction mixture was heated to reflux for 3 hours. After cooling to room temperature, the mixture was concentrated under reduced pressure and the solid was triturated with $26 \%$ aqueous solution of ammonia. The precipitate was filtered under reduced pressure and washed with cold $96 \%$ ethanol to afford 2,2'-bibenzothiazole as a gold-yellow solid $(7.0 \mathrm{~g})$ in $70 \%$ yield.

${ }^{1}$ H NMR $\left(600 \mathrm{MHz}, \mathrm{CDCl}_{3}\right) \delta 8.17(\mathrm{~d}, J=8.2 \mathrm{~Hz}, 2 \mathrm{H}), 7.99(\mathrm{~d}, J=8.3 \mathrm{~Hz}, 2 \mathrm{H}), 7.57$ (t, $J=7.8$ $\mathrm{Hz}, 2 \mathrm{H}), 7.50(\mathrm{t}, J=7.7 \mathrm{~Hz}, 2 \mathrm{H}) .{ }^{13} \mathbf{C} \mathbf{N M R}\left(151 \mathrm{MHz}, \mathrm{CDCl}_{3}\right) \delta 161.5,153.6,135.9,126.8$, 126.6, 124.1, 122.0. 


\subsection{Homocoupling of arylamine-functionalized 2-H-benzothiazoles - general procedure}

Given arylamine-functionalized 2-H-benzothiazole $(2 \mathrm{mmol})$ was dissolved in a mixture of xylenes $(20 \mathrm{~mL})$, heated to reflux and $\mathrm{Cu}(\mathrm{OAc})_{2}$ (1.0 equivalent) was added portionwise over the course of 10 minutes [note: $\mathrm{Cu}(\mathrm{OAc})_{2}$ was dried under reduced pressure at $155^{\circ} \mathrm{C}$ for 3 hours]. After the addition was complete, the reaction mixture was heated to reflux for $2-3$ hours. Then the reaction mixture was concentrated under reduced pressure, slurry dissolved in dichloromethane $(60 \mathrm{~mL})$, the organic layer washed with hot saturated aq. solution of disodium EDTA $(60 \mathrm{~mL})$, brine $(30 \mathrm{~mL})$ and dried over anhydrous $\mathrm{Na}_{2} \mathrm{SO}_{4}$. Organic solvent was evaporated under reduced pressure and the solid residue purified by column chromatography or crystalized.

\subsubsection{6,6'-Bis $(N, N$-diphenylamino)-2,2'-bibenzothiazole (bisbtz-I)}

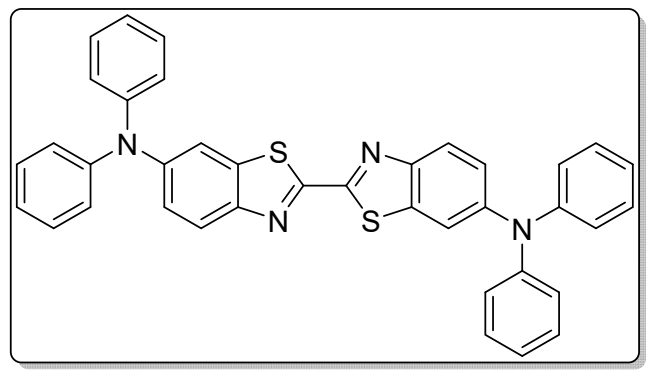

(yellow solid) Eluent: hexanes / $\mathrm{CHCl}_{3} 1: 1 ;\left(\mathrm{SiO}_{2}, \mathbf{R}_{\mathbf{f}}\right.$ $=0.18)$, yield: $0.43 \mathrm{~g}(72 \%)$. Mp $295-297{ }^{\circ} \mathrm{C} .{ }^{\mathbf{1}} \mathbf{H}$ NMR $\left(600 \mathrm{MHz}, \mathrm{CDCl}_{3}\right) \delta 7.93(\mathrm{~d}, J=8.9 \mathrm{~Hz}, 2 \mathrm{H})$, $7.53(\mathrm{~d}, J=2.3 \mathrm{~Hz}, 2 \mathrm{H}), 7.34-7.28(\mathrm{~m}, 8 \mathrm{H}), 7.27$ (d, $J=2.2 \mathrm{~Hz}, 1 \mathrm{H}), 7.26(\mathrm{~d}, J=2.3 \mathrm{~Hz}, 1 \mathrm{H}), 7.15(\mathrm{~d}, J=$ $7.4 \mathrm{~Hz}, 8 \mathrm{H}), 7.10(\mathrm{t}, J=7.4 \mathrm{~Hz}, 4 \mathrm{H}) .{ }^{13} \mathbf{C}$ NMR $(151$ $\left.\mathrm{MHz}, \mathrm{CDCl}_{3}\right) \delta 159.5,149.1,147.4,147.0,137.2,129.5,124.9,124.1,123.7,123.3,114.7 .{ }^{15} \mathbf{N}$ NMR $\left(61 \mathrm{MHz}, \mathrm{CDCl}_{3}\right) \delta$-279.3, -69.8. Anal. Calcd for $\mathrm{C}_{38} \mathrm{H}_{26} \mathrm{~N}_{4} \mathrm{~S}_{2}$ : C, 75.72; H, 4.35; N, 9.30. Found: C, 75.53; H, 4.39; N, 9.40. IR (ATR): v 2924 (w), 2115 (w), 1583 (s), 1484 (s), 1468 (s), 1269 (s), 753 (s), 694 (s) $\mathrm{cm}^{-1}$.

\subsubsection{6,6'-Bis [4-(N,N-diphenylamino)phenyl]-2,2'-bibenzothiazole (bisbtz-II)}

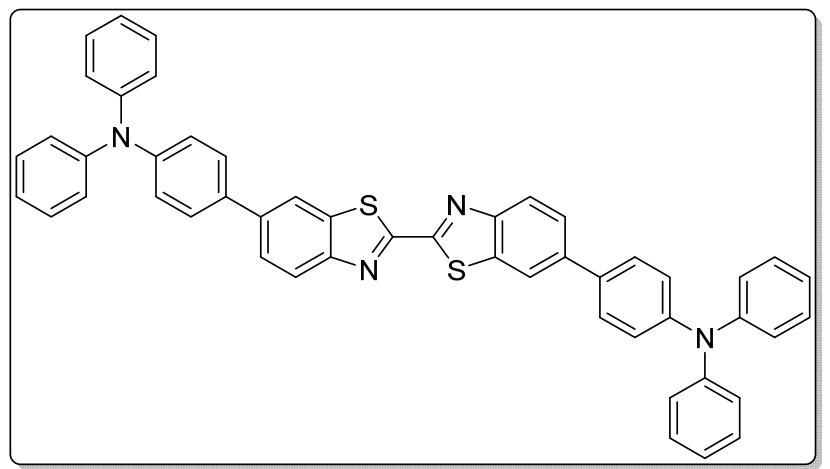

(orange solid) Crystalized from chloroform and hexanes, yield: $0.51 \mathrm{~g}(68 \%)$. Mp 276 $-278{ }^{\circ} \mathrm{C} .{ }^{1} \mathbf{H}$ NMR $\left(600 \mathrm{MHz}, \mathrm{CDCl}_{3}\right) \delta$ $8.17(\mathrm{~d}, J=8.6 \mathrm{~Hz}, 2 \mathrm{H}), 8.15(\mathrm{~d}, J=1.9$ $\mathrm{Hz}, 2 \mathrm{H}), 7.77$ (dd, $J=8.6,1.9 \mathrm{~Hz}, 2 \mathrm{H})$, $7.56(\mathrm{~d}, J=8.7 \mathrm{~Hz}, 4 \mathrm{H}), 7.31-7.28(\mathrm{~m}$, $8 \mathrm{H}), 7.17(\mathrm{t}, J=8.7 \mathrm{~Hz}, 12 \mathrm{H}), 7.08-7.05$

$(\mathrm{m}, 4 \mathrm{H}) .{ }^{13} \mathbf{C} \mathbf{N M R}\left(151 \mathrm{MHz}, \mathrm{CDCl}_{3}\right) \delta 161.2,152.6,147.8,147.5,139.5,136.7,133.7,129.4$, 128.1, 126.1, 124.7, 124.1, 123.6, 123.2, 119.4. Anal. Calcd for $\mathrm{C}_{50} \mathrm{H}_{34} \mathrm{~N}_{4} \mathrm{~S}_{2}$ : C, 79.55; H, 4.54; N, 7.42. Found: C, 79.77; H, 4.43; N, 7.27. IR (ATR): v 2331 (w), 2126 (w), 1922 (w), 1587 (s), 1489 (s), 1326 (s), $1268(\mathrm{~s}), 815(\mathrm{~s}) \mathrm{cm}^{-1}$. 


\subsubsection{6,6'-Bis $\{4-[N, N$-bis(4-methoxyphenyl)amino]phenyl $\}-2,2$ '-bibenzothiazole}

(bisbtz-III)

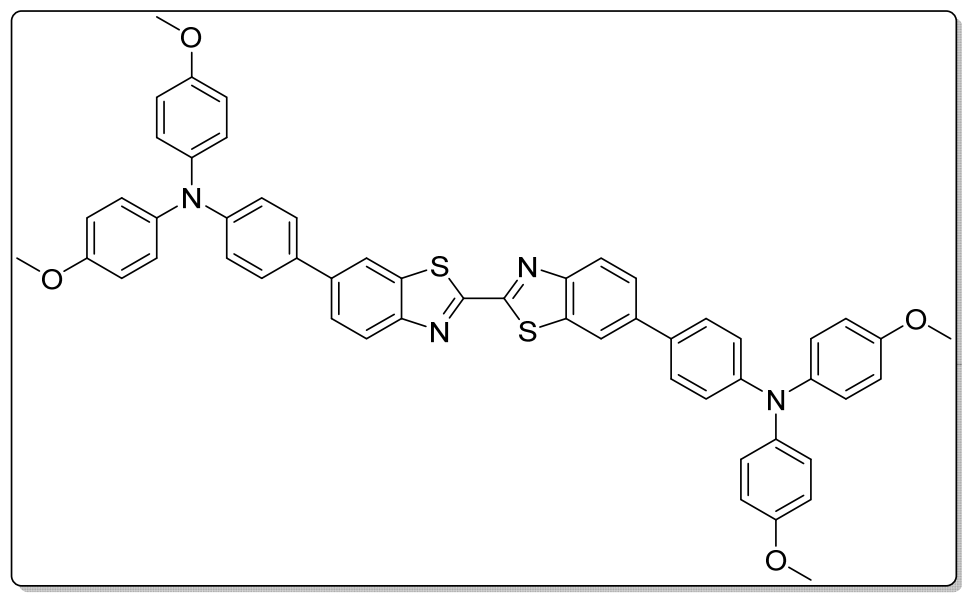

(red solid) Eluent: hexanes / $\mathrm{CHCl}_{3} 4: 1\left(\mathrm{SiO}_{2}, \mathbf{R}_{\mathbf{f}}=0.2\right)$, yield: 0.84 g $(96 \%)$. Mp $279-281{ }^{\circ} \mathrm{C}$. ${ }^{1} \mathbf{H}$ NMR $\left(600 \mathrm{MHz}, \mathrm{CDCl}_{3}\right) \delta$ $8.15(\mathrm{~d}, J=8.6 \mathrm{~Hz}, 2 \mathrm{H}), 8.11(\mathrm{~d}$, $J=1.4 \mathrm{~Hz}, 2 \mathrm{H}), 7.75(\mathrm{dd}, J=8.6$, $1.7 \mathrm{~Hz}, 2 \mathrm{H}), 7.50(\mathrm{~d}, J=8.7 \mathrm{~Hz}$, 4H), $7.11(\mathrm{~d}, J=8.9 \mathrm{~Hz}, 8 \mathrm{H}), 7.02$ $(\mathrm{d}, J=8.7 \mathrm{~Hz}, 4 \mathrm{H}), 6.86(\mathrm{~d}, J=$ $8.9 \mathrm{~Hz}, 8 \mathrm{H}), 3.82(\mathrm{~s}, 12 \mathrm{H}) .{ }^{13} \mathbf{C}$ NMR $\left(151 \mathrm{MHz}, \mathrm{CDCl}_{3}\right) \delta 161.0,156.1,152.4,149.1,140.6$, 139.7, 136.7, 131.6, 127.8, 126.8, 126.0, 124.0, 120.8, 119.1, 115.1, 55.5. Anal. Calcd for $\mathrm{C}_{54} \mathrm{H}_{42} \mathrm{~N}_{4} \mathrm{O}_{4} \mathrm{~S}_{2}$ : C, 74.12; H, 4.84; N, 6.40. Found: C, 74.32; H, 4.50; N, 6.47. IR (ATR): v 2355 (w), 1591 (s), 1503 (m), 1460 (s), 1238 (s), 1104 (m), 1028 (s), 810 (s) cm . $^{-1}$

\subsubsection{6,6'-Bis [4-( $N, N$-diphenylamino)phenylethynyl]-2,2'-bibenzothiazole (bisbtz-IV)}

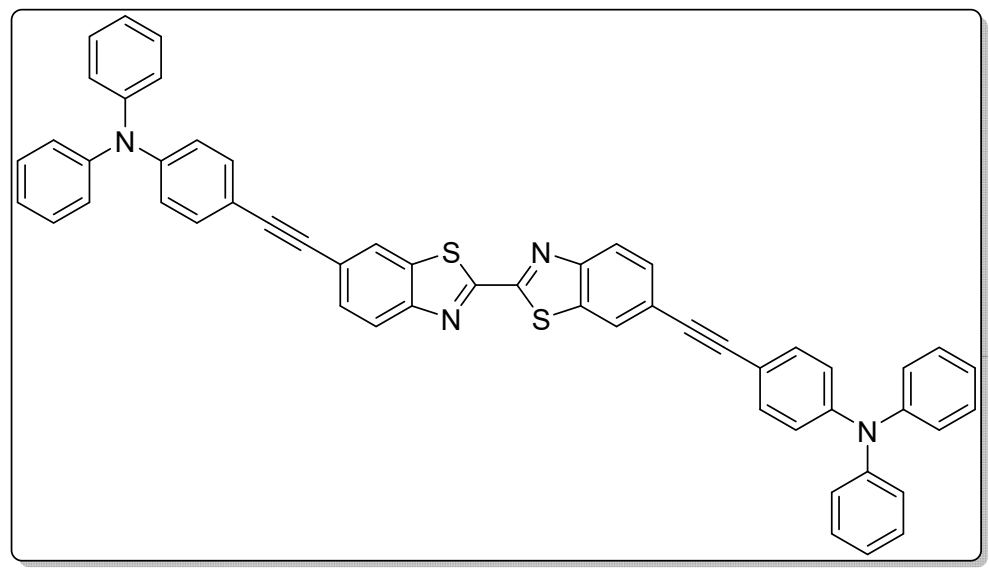

(orange solid) Eluent: hexanes / $\mathrm{CHCl}_{3}$ 1:1 $\left(\mathrm{SiO}_{2}, \mathbf{R}_{\mathbf{f}}=0.25\right)$, yield: $0.50 \mathrm{~g}(62 \%)$. Mp $300-$ $302{ }^{\circ} \mathrm{C} .{ }^{1} \mathbf{H}$ NMR $(600 \mathrm{MHz}$, $\left.\mathrm{CDCl}_{3}\right) \delta 8.12(\mathrm{~d}, J=1.7 \mathrm{~Hz}$, $2 \mathrm{H}), 8.09(\mathrm{~d}, J=8.4 \mathrm{~Hz}, 2 \mathrm{H})$, $7.67(\mathrm{dd}, J=8.5,1.7 \mathrm{~Hz}, 2 \mathrm{H})$, $7.40(\mathrm{~d}, J=8.8 \mathrm{~Hz}, 4 \mathrm{H}), 7.31-$ $7.28(\mathrm{~m}, 8 \mathrm{H}), 7.13(\mathrm{~d}, J=7.5 \mathrm{~Hz}$, 8H), $7.08(\mathrm{t}, J=7.4 \mathrm{~Hz}, 4 \mathrm{H}), 7.02(\mathrm{~d}, J=8.8 \mathrm{~Hz}, 4 \mathrm{H}) .{ }^{13} \mathbf{C ~ N M R}\left(151 \mathrm{MHz}, \mathrm{CDCl}_{3}\right) \delta 161.9$, $152.9,148.3,147.1,136.1,132.6,130.4,129.4,125.1,124.8,123.8,123.7,122.4,122.1,115.4$, 91.8, 88.2. Anal. Calcd for $\mathrm{C}_{54} \mathrm{H}_{34} \mathrm{~N}_{4} \mathrm{~S}_{2}$ : C, 80.77; H, 4.27; N, 6.98. Found: C, 80.71; H, 4.42; $\mathrm{N}$, 7.00. IR (ATR): v 2920 (w), 2852 (w), 2360 (w), 2338 (w), 1581 (s), 1487 (s), 1285 (s), 817 (s) $\mathrm{cm}^{-1}$. 


\subsubsection{6,6'-Bis $\{4-[N, N$-Bis(4-methoxyphenyl)amino]phenylethynyl $\}$-2,2'-bibenzothiazole (bisbtz-V)}

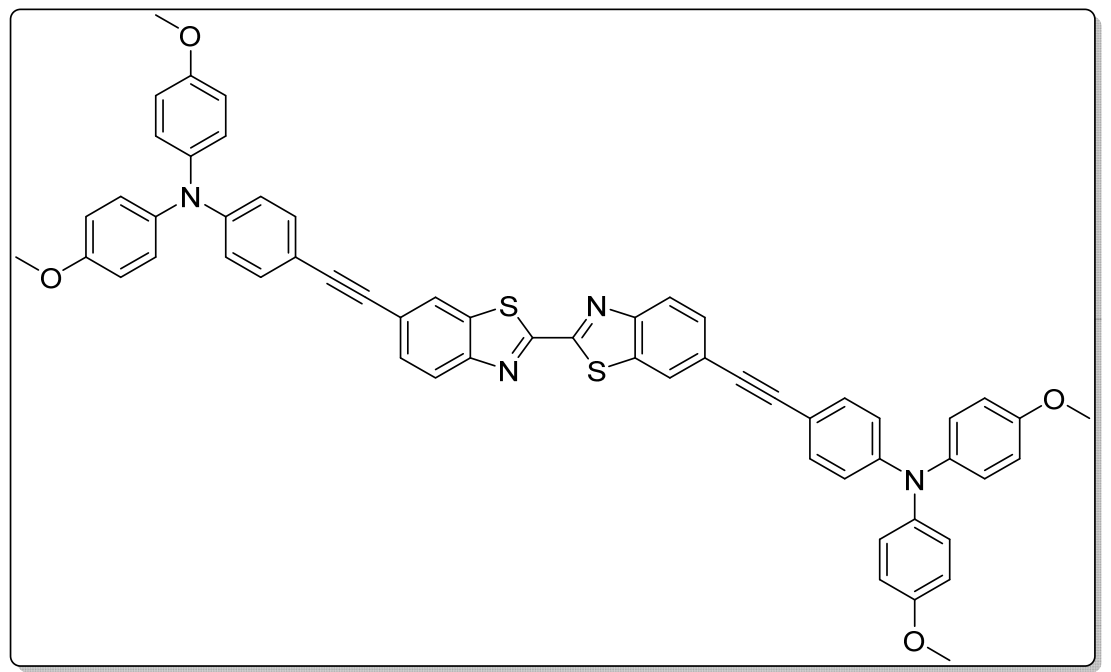

(orange solid) Eluent: hexanes / $\mathrm{CHCl}_{3} \quad 4: 1$ $\left(\mathrm{SiO}_{2}, \mathbf{R}_{\mathbf{f}}=0.20\right)$, yield: 0.83 g $(90 \%)$. Мp $223-$ $225{ }^{\circ} \mathrm{C} .{ }^{1} \mathbf{H}$ NMR $(600$ $\left.\mathrm{MHz}, \mathrm{CDCl}_{3}\right) \delta 8.11(\mathrm{~d}$, $\mathrm{J}=6 \mathrm{~Hz}, 2 \mathrm{H}), 8.07(\mathrm{~d}, \mathrm{~J}=$ $8.5 \mathrm{~Hz}, 2 \mathrm{H}), 7.65(\mathrm{dd}, J$ $=8.5,1.3 \mathrm{~Hz}, 2 \mathrm{H}), 7.34$ $(\mathrm{d}, J=8.7 \mathrm{~Hz}, 4 \mathrm{H}), 7.09$ $(\mathrm{d}, J=8.9 \mathrm{~Hz}, 8 \mathrm{H}), 6.86(\mathrm{~d}, J=8.9 \mathrm{~Hz}, 12 \mathrm{H}), 3.81(\mathrm{~s}, 12 \mathrm{H}) \cdot{ }^{13} \mathbf{C}$ NMR $\left(151 \mathrm{MHz}, \mathrm{CDCl}_{3}\right) \delta$ $161.8,157.2$, 152.3, 149.1, 140.0, 136.1, 132.5, 130.3, 127.2, 124.6, 123.7, 119.0, 114.8, 113.2, 92.3, 55.5. Anal. Calcd for $\mathrm{C}_{58} \mathrm{H}_{42} \mathrm{~N}_{4} \mathrm{O}_{4} \mathrm{~S}_{2}$ : C, 75.47; H, 4.59; N, 6.07. Found: C, 75.41; H, 4.75; N, 5.98. IR (ATR): v 2835 (w), 2341 (w), 2199 (w), 1585 (s), 1500 (s), 1283 (s), 1239 (s), 819 (s) $\mathrm{cm}^{-1}$.

\subsection{6 (E,E)-6,6'-[4-( $N, N$-Diphenylamino)phenylethenyl]-2,2'-bibenzothiazole (bisbtz-VI)}

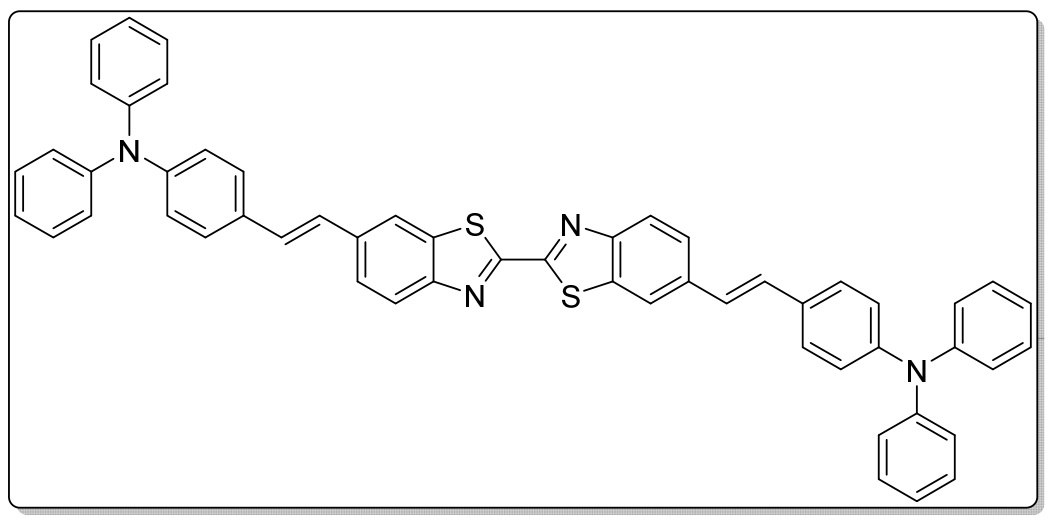

(red solid) Crystalized from DCM and hexanes, yield: 0.65 g $(80 \%)$. Mp $290-292$ ${ }^{\circ} \mathrm{C}$. ${ }^{1} \mathbf{H}$ NMR $(600 \mathrm{MHz}$, $\left.\mathrm{CDCl}_{3}\right) \delta 8.09(\mathrm{~d}, J=8.6 \mathrm{~Hz}$, $2 \mathrm{H}), 8.04(\mathrm{~d}, J=1.7 \mathrm{~Hz}$, $2 \mathrm{H}), 7.71(\mathrm{dd}, J=8.7,1.7$ $\mathrm{Hz}, 2 \mathrm{H}), 7.42(\mathrm{~d}, J=8.7 \mathrm{~Hz}$, 4H), $7.30-7.26(\mathrm{~m}, 8 \mathrm{H}), 7.21-7.12(\mathrm{~m}, 12 \mathrm{H}), 7.09-7.04(\mathrm{~m}, 8 \mathrm{H}) .{ }^{13} \mathbf{C} \mathbf{N M R}\left(151 \mathrm{MHz}, \mathrm{CDCl}_{3}\right)$ $\delta 161.1,153.0,147.9,147.5,143.9,136.8,136.7,130.6,129.9,129.3,127.6,125.9,125.4,124.7$, 124.0, 123.3, 123.3, 119.2. Anal. Calcd for $\mathrm{C}_{54} \mathrm{H}_{38} \mathrm{~N}_{4} \mathrm{~S}_{2}$ : C, 80.37; H, 4.75; N, 6.94. Found: C, 80.50; H, 4.71; N, 6.88. IR (ATR): v 3021 (w), 2977 (w), 1583 (s), 1487 (s), 1324 (s), 1274 (s), 1174 (s), 794 (s) $\mathrm{cm}^{-1}$. 


\section{Complexation of bisbtz-I with $\operatorname{ReCl}(\mathrm{CO})_{5}$}

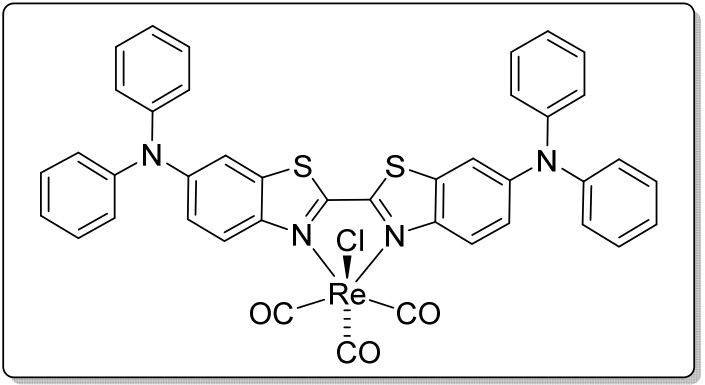

A mixture of bisbtz-I (75 $\mathrm{mg}, 0.12 \mathrm{mmol})$ and rhenium pentacarbonyl chloride $(45 \mathrm{mg}, 0.12$ mmol) was dissolved in dry toluene $(5 \mathrm{~mL})$ and heated to reflux for $1 \mathrm{~h}$ under an inert atmosphere. The reaction mixture was cooled to room temperature and concentrated under reduced

pressure. The solid residue was dissolved in hot chloroform, filtered and concentrated under reduced pressure, affording $\operatorname{ReCl}(\mathrm{CO})_{3}($ bisbtz-I) as a dark purple solid $(80 \mathrm{mg})$ in $75 \%$ yield. $\mathbf{M p}$ $>220{ }^{\circ} \mathrm{C} .{ }^{1} \mathbf{H}$ NMR $\left(600 \mathrm{MHz}, \mathrm{CDCl}_{3}\right) \delta 8.31(\mathrm{~d}, J=9.2 \mathrm{~Hz}, 2 \mathrm{H}), 7.45(\mathrm{dd}, J=9.2,2.2 \mathrm{~Hz}, 2 \mathrm{H})$, $7.42(\mathrm{~d}, J=2.2 \mathrm{~Hz}, 2 \mathrm{H}), 7.37(\mathrm{t}, J=7.9 \mathrm{~Hz}, 8 \mathrm{H}), 7.21-7.18(\mathrm{~m}, 12 \mathrm{H}) .{ }^{13} \mathbf{C} \mathbf{N M R}(151 \mathrm{MHz}$, $\left.\mathrm{CDCl}_{3}\right) \delta 158.40,149.32,146.36,145.51,136.06,129.89,125.76,125.07,124.26,124.21,112.22$. ${ }^{15} \mathrm{~N}$ NMR $\left(61 \mathrm{MHz}, \mathrm{CDCl}_{3}\right) \delta$-274.6, -123.7. Anal. Calcd for $\mathrm{C}_{41} \mathrm{H}_{26} \mathrm{ClN}_{3} \mathrm{O}_{3} \mathrm{ReS}_{2}: \mathrm{C}, 55.06 ; \mathrm{H}$, 2.93; N, 4.90. Found: C, 55.12; H, 2.89; N, 4.73. IR (ATR): v 2016 (s), 1891 (s), 1578 (s), 1544 (s), $1544(\mathrm{~m}), 1486(\mathrm{~s}), 1467$ (s), 1288 (s), 1258 (s), 693 (s) cm $\mathrm{cm}^{-1}$. 
6 NMR spectra of key intermediates and target 2,2'-bibenzothiazoles

${ }^{1} \mathrm{H}$ NMR spectrum of 6-iodobenzothiazol-2(3H)-one (600 $\left.\mathrm{MHz}, \mathrm{CDCl}_{3}\right)$
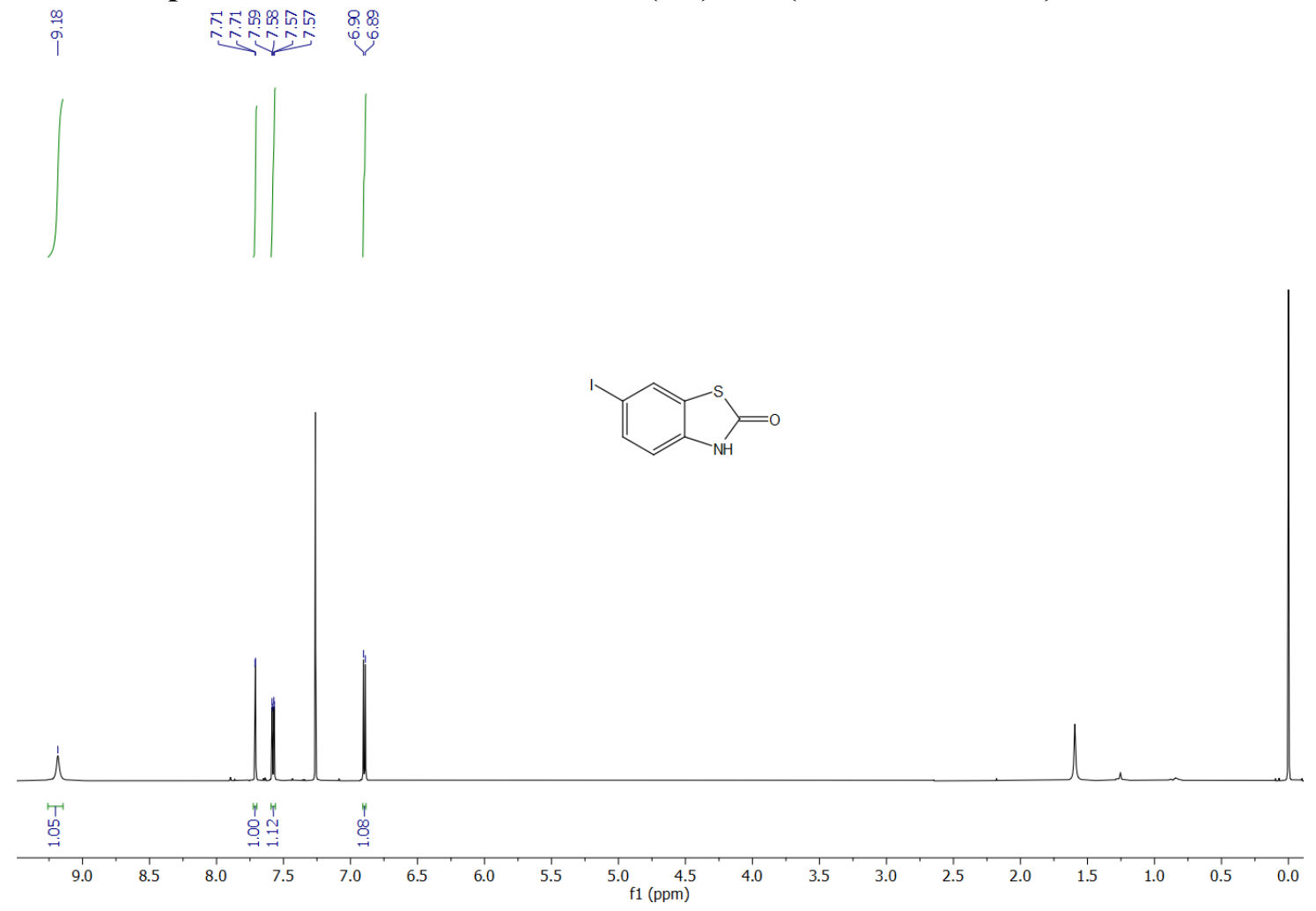

${ }^{13} \mathrm{C}$ NMR spectrum of 6-iodobenzothiazol-2(3H)-one (151 $\left.\mathrm{MHz}, \mathrm{CDCl}_{3}\right)$
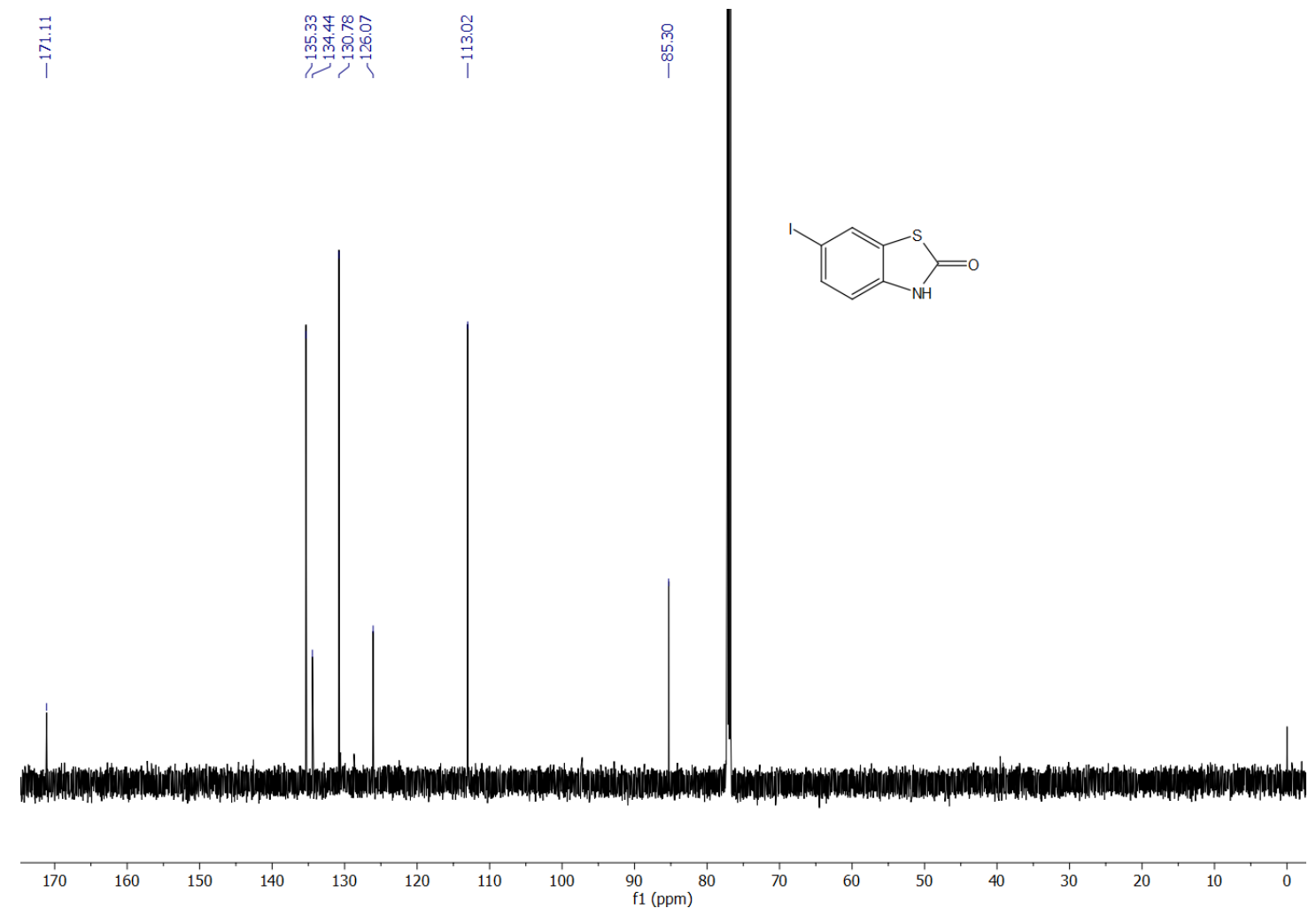
${ }^{1} \mathrm{H}$ NMR spectrum of 2 -chloro-6-iodobenzothiazole (600 $\left.\mathrm{MHz}, \mathrm{CDCl}_{3}\right)$

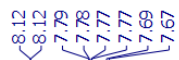
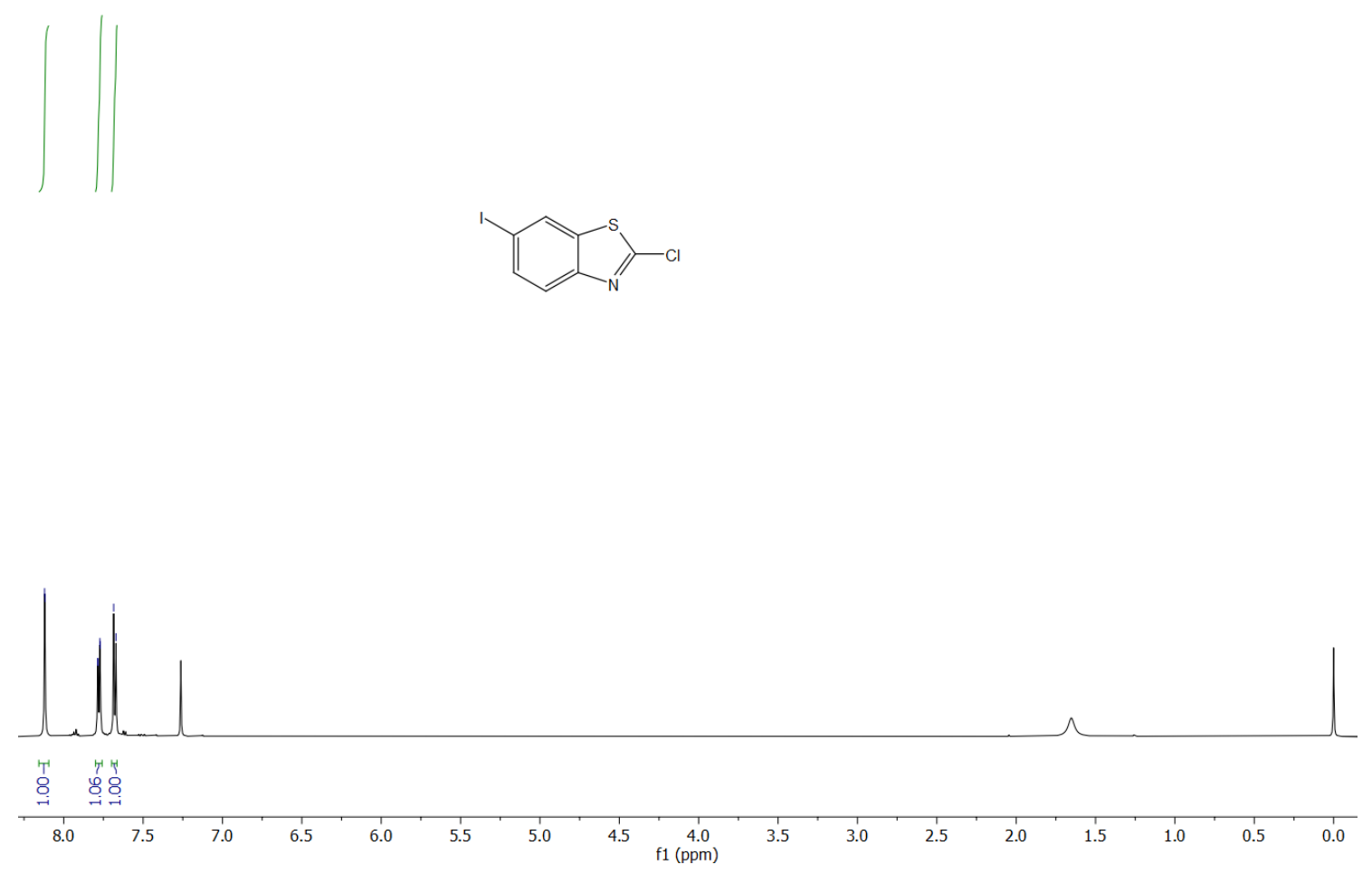

${ }^{13} \mathrm{C}$ NMR spectrum of 2-chloro-6-iodobenzothiazole (151 $\left.\mathrm{MHz}, \mathrm{CDCl}_{3}\right)$
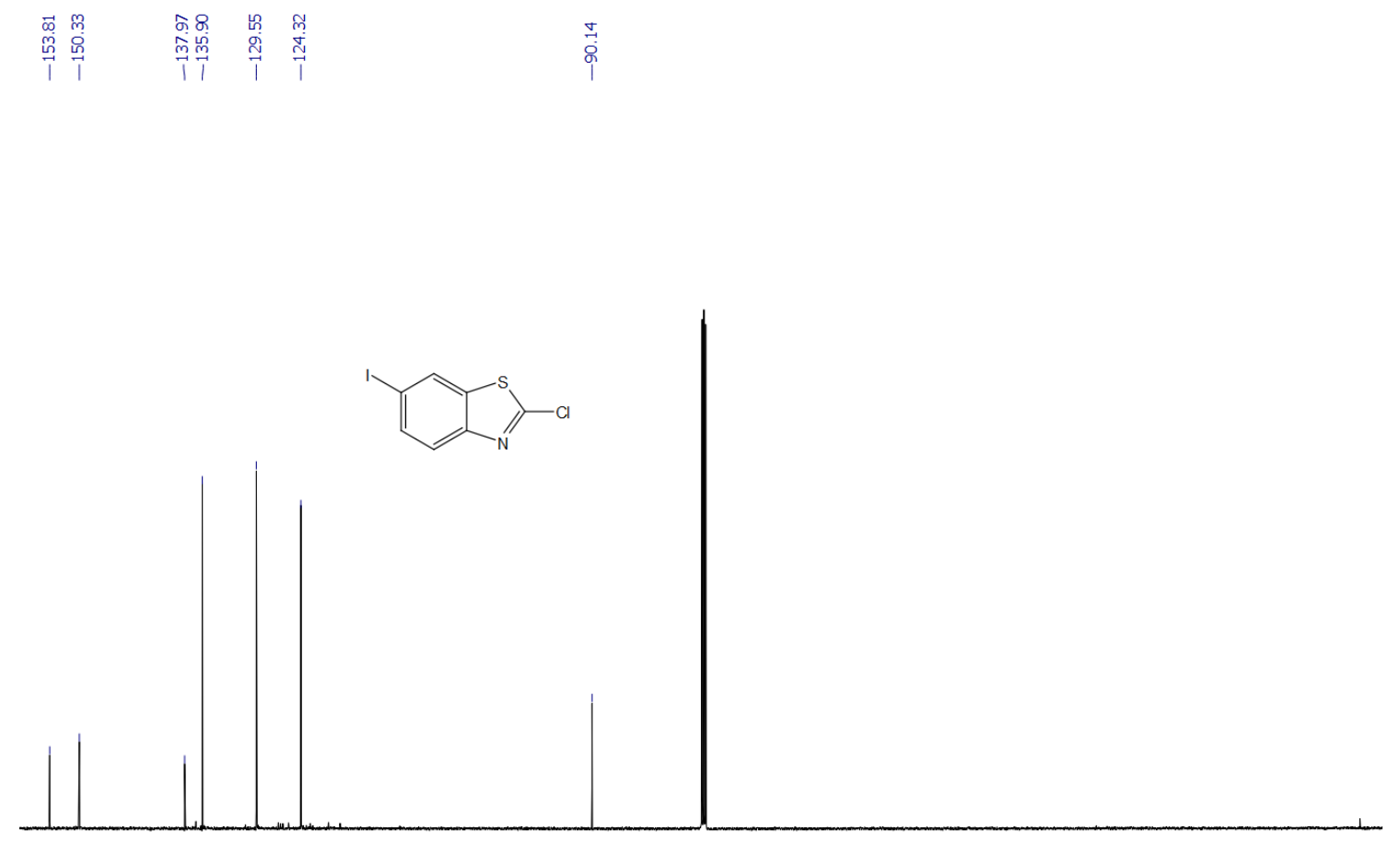
${ }^{1} \mathrm{H}$ NMR spectrum of 6-iodobenzothiazole (600 $\left.\mathrm{MHz}, \mathrm{CDCl}_{3}\right)$
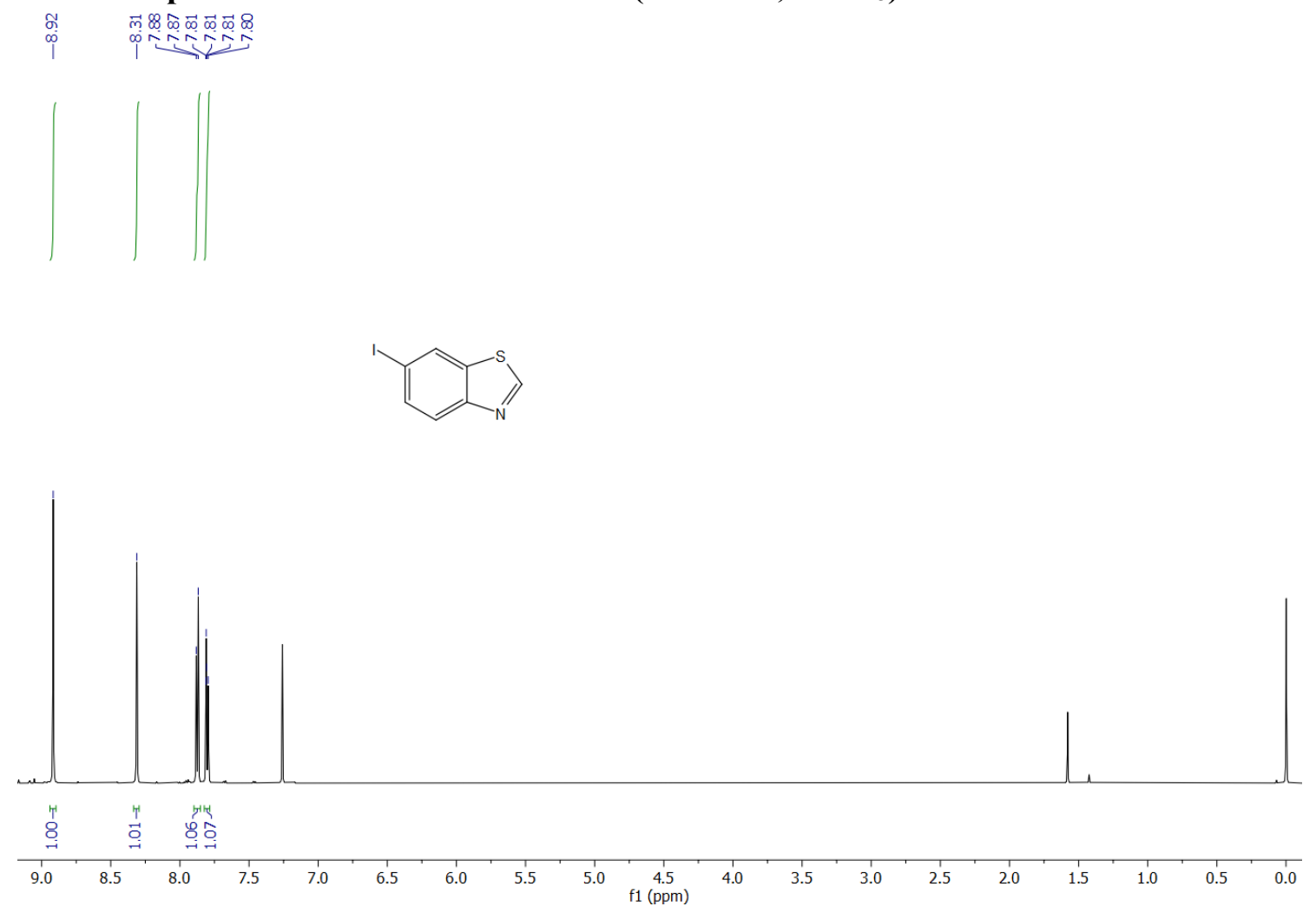

${ }^{13} \mathrm{C}$ NMR spectrum of 6-iodobenzothiazole (151 $\mathrm{MHz}, \mathrm{CDCl}_{3}$ )
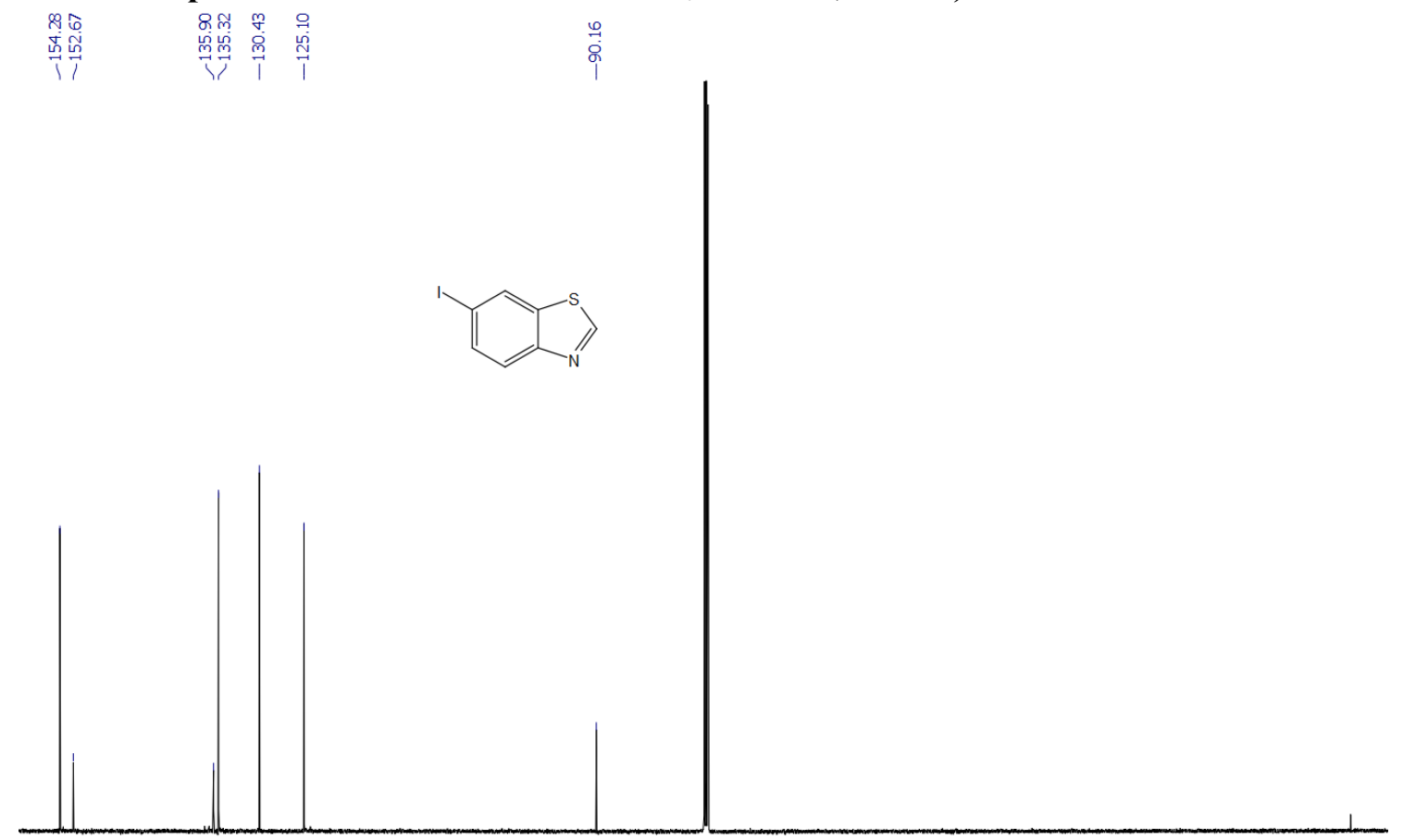
${ }^{1} \mathrm{H}$ NMR spectrum of 6-nitrobenzothiazole (600 $\left.\mathrm{MHz}, \mathrm{CDCl}_{3}\right)$
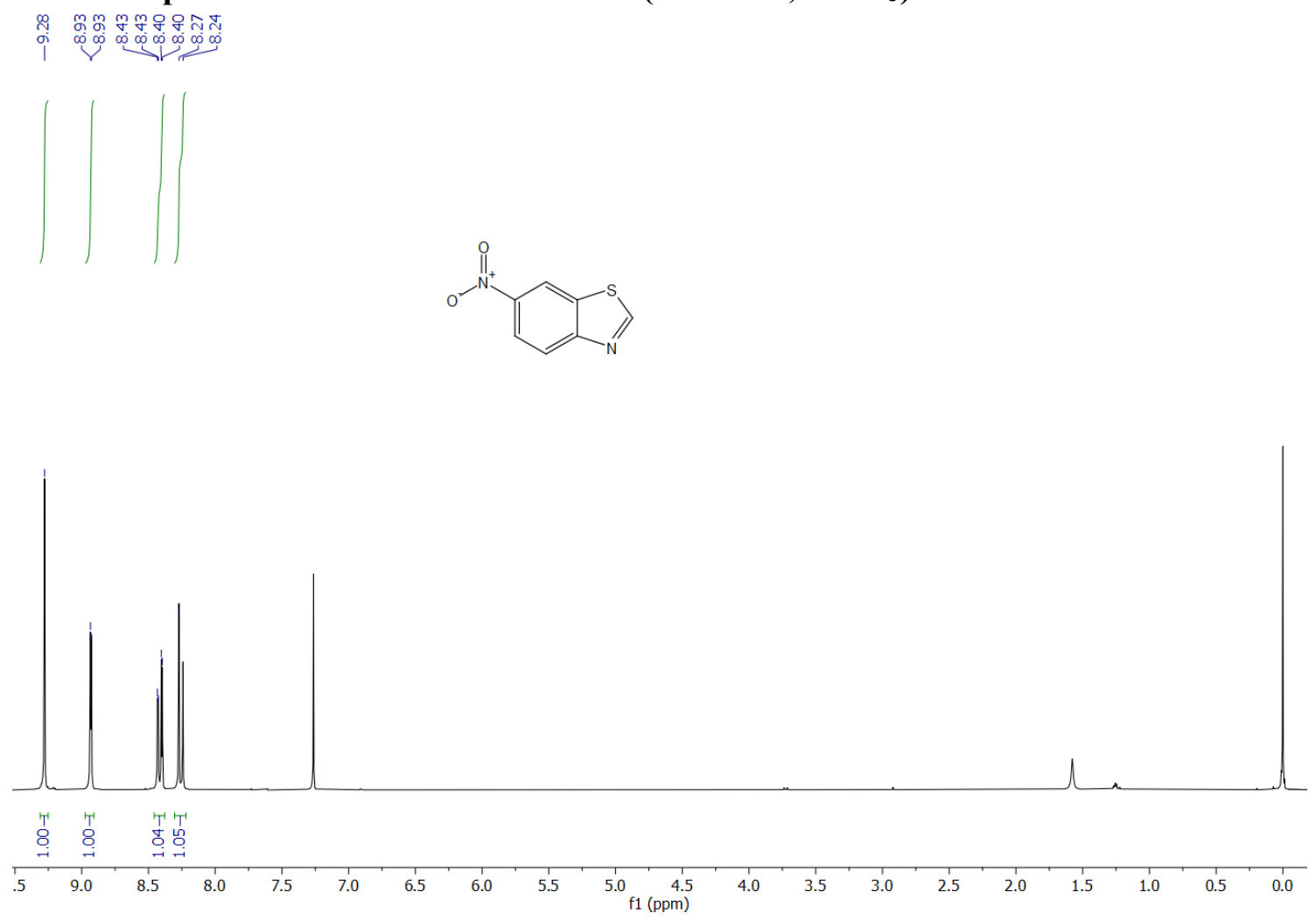

${ }^{1} \mathrm{H}$ NMR spectrum of benzothiazol-6-amine (600 MHz, DMSO- $\left.d_{6}\right)$

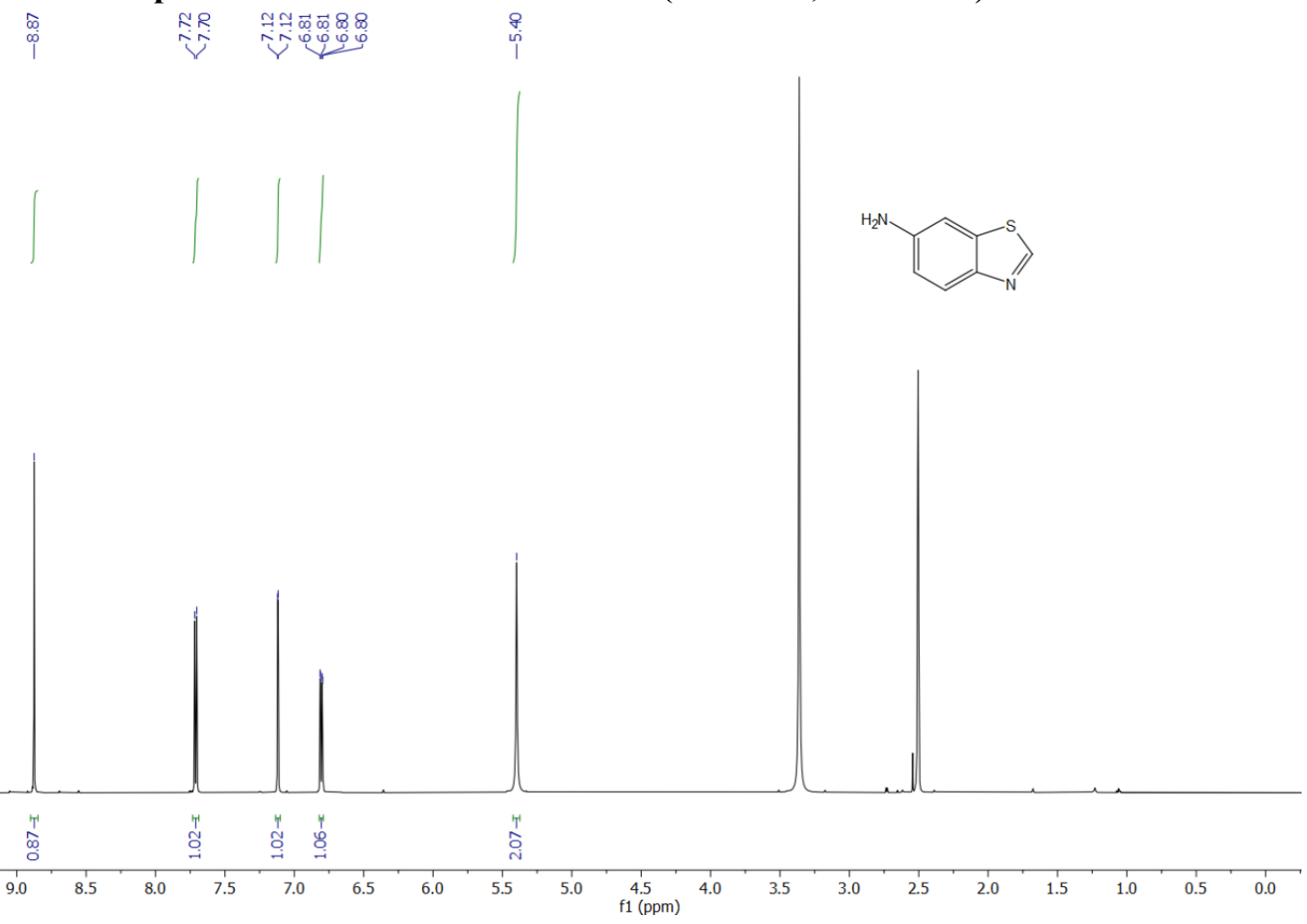

S30 | Supporting Information 
${ }^{1} \mathrm{H}$ NMR spectrum of 6-[ $N, N$-bis(4-nitrophenyl)amino]benzothiazole (600 MHz, DMSO-d 6 )

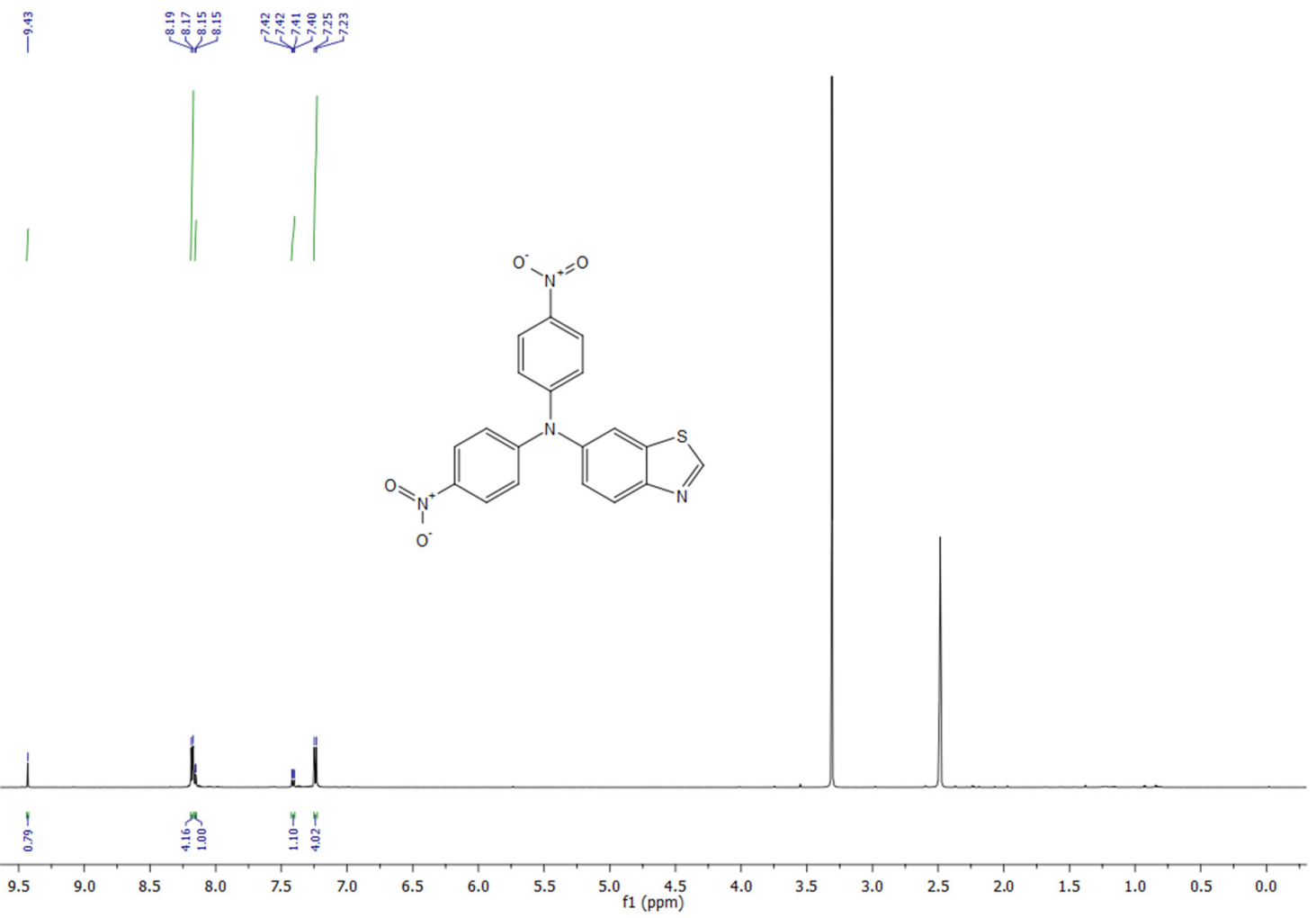

${ }^{13} \mathrm{C}$ NMR spectrum of 6-[N,N-bis(4-nitrophenyl)amino]benzothiazole (151 MHz, DMSO-d 6 )
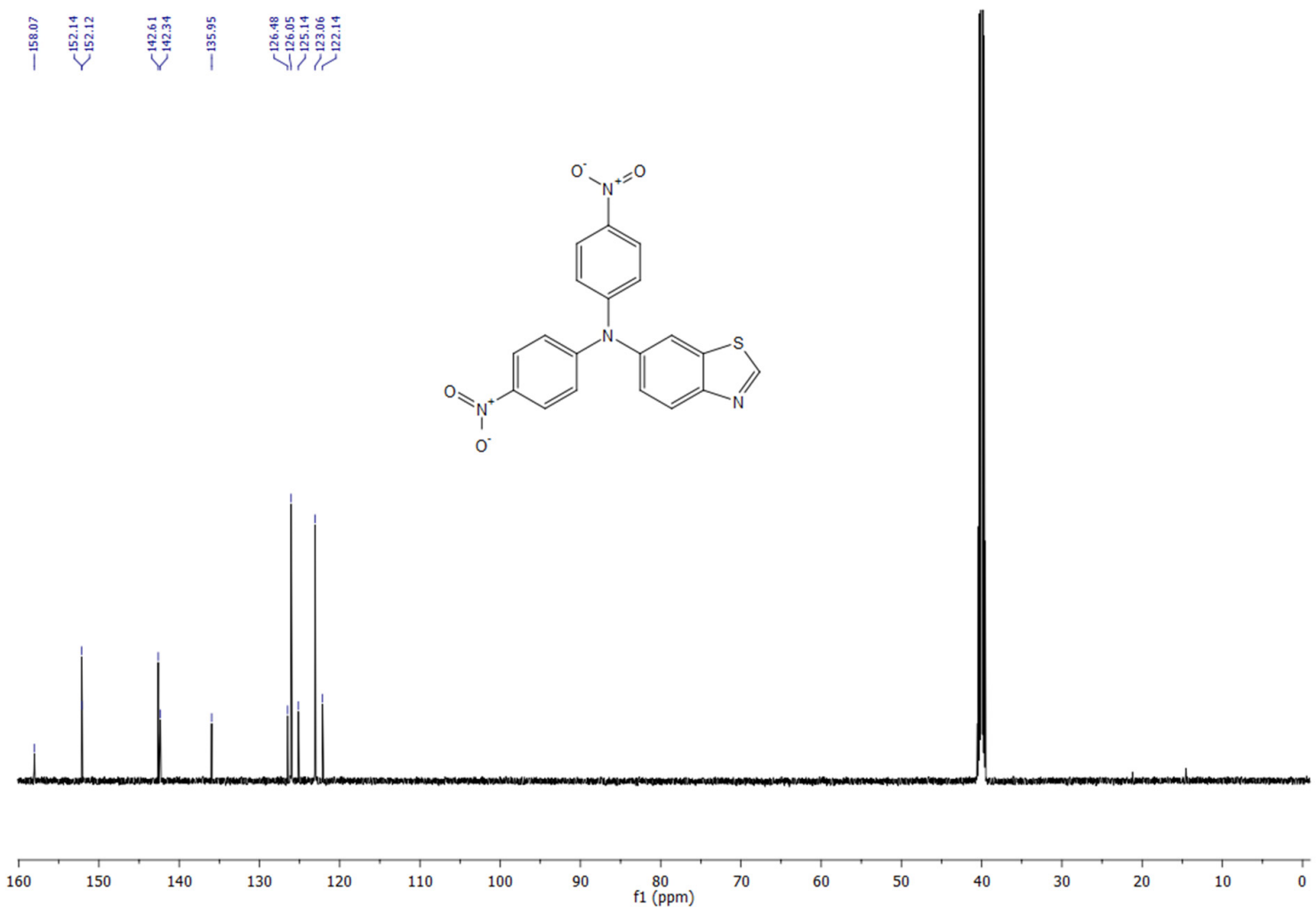
${ }^{1}$ H NMR spectrum of 6-[ $N, N$-bis(4-aminophenyl)amino]benzothiazole (600 MHz, DMSO- $d_{6}$ )

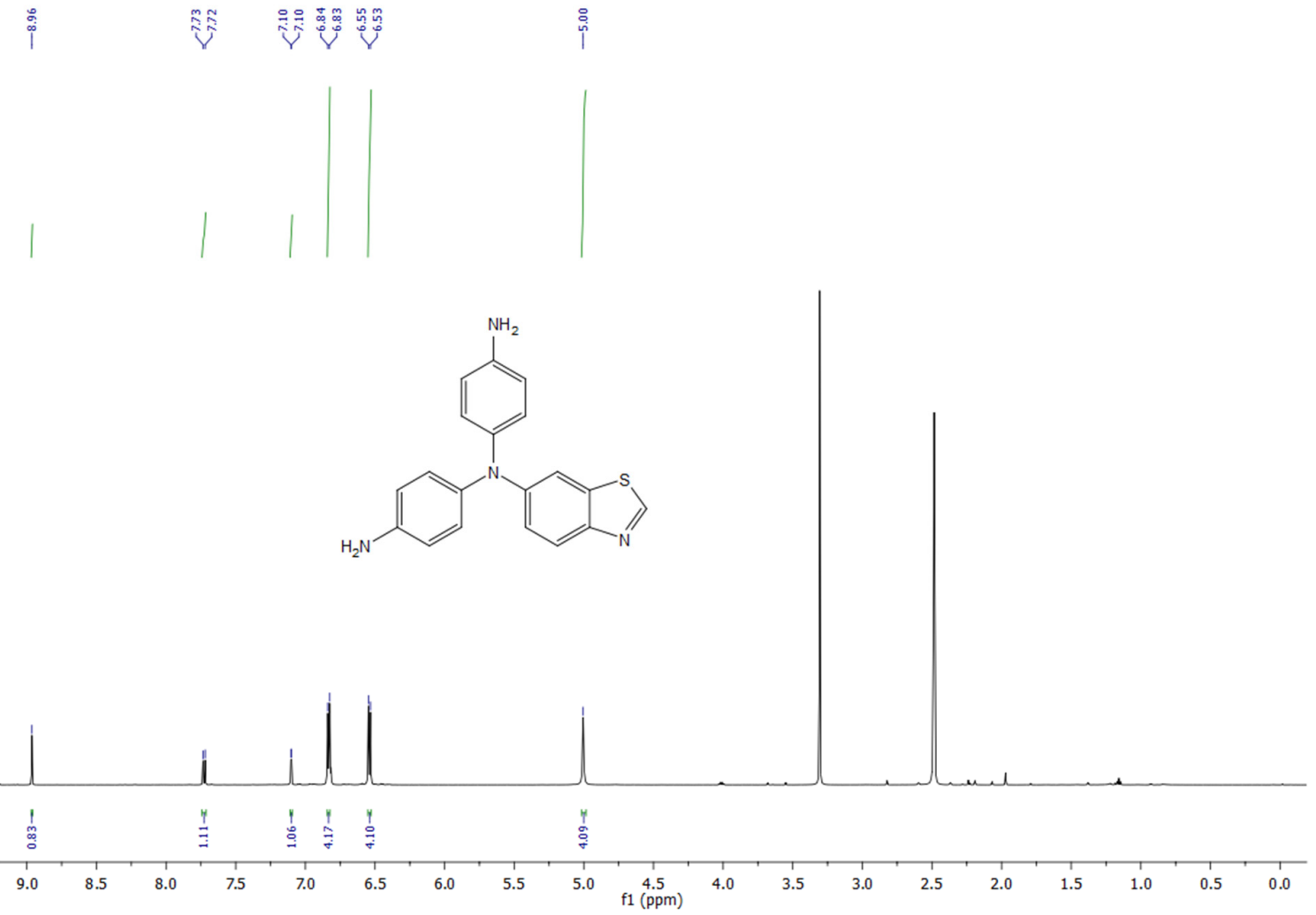

${ }^{13} \mathrm{C}$ NMR spectrum of 6-[N, $N$-bis(4-aminophenyl)amino]benzothiazole (151 MHz, DMSO-d 6 )

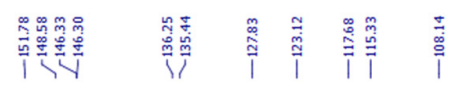
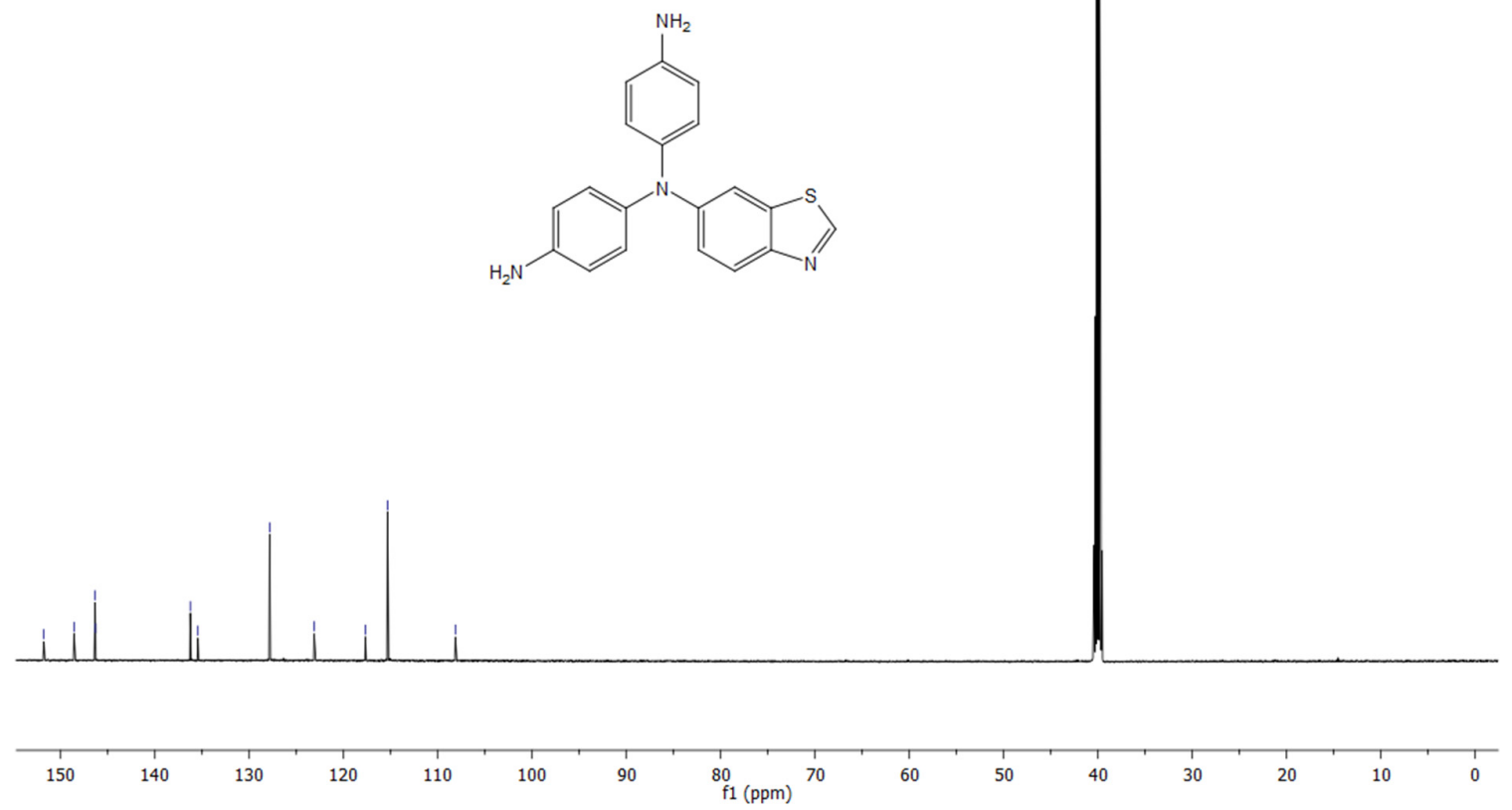
${ }^{1} \mathrm{H}$ NMR spectrum of diphenyliodonium triflate (600 MHz, DMSO- $\left.d_{6}\right)$

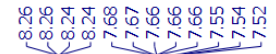

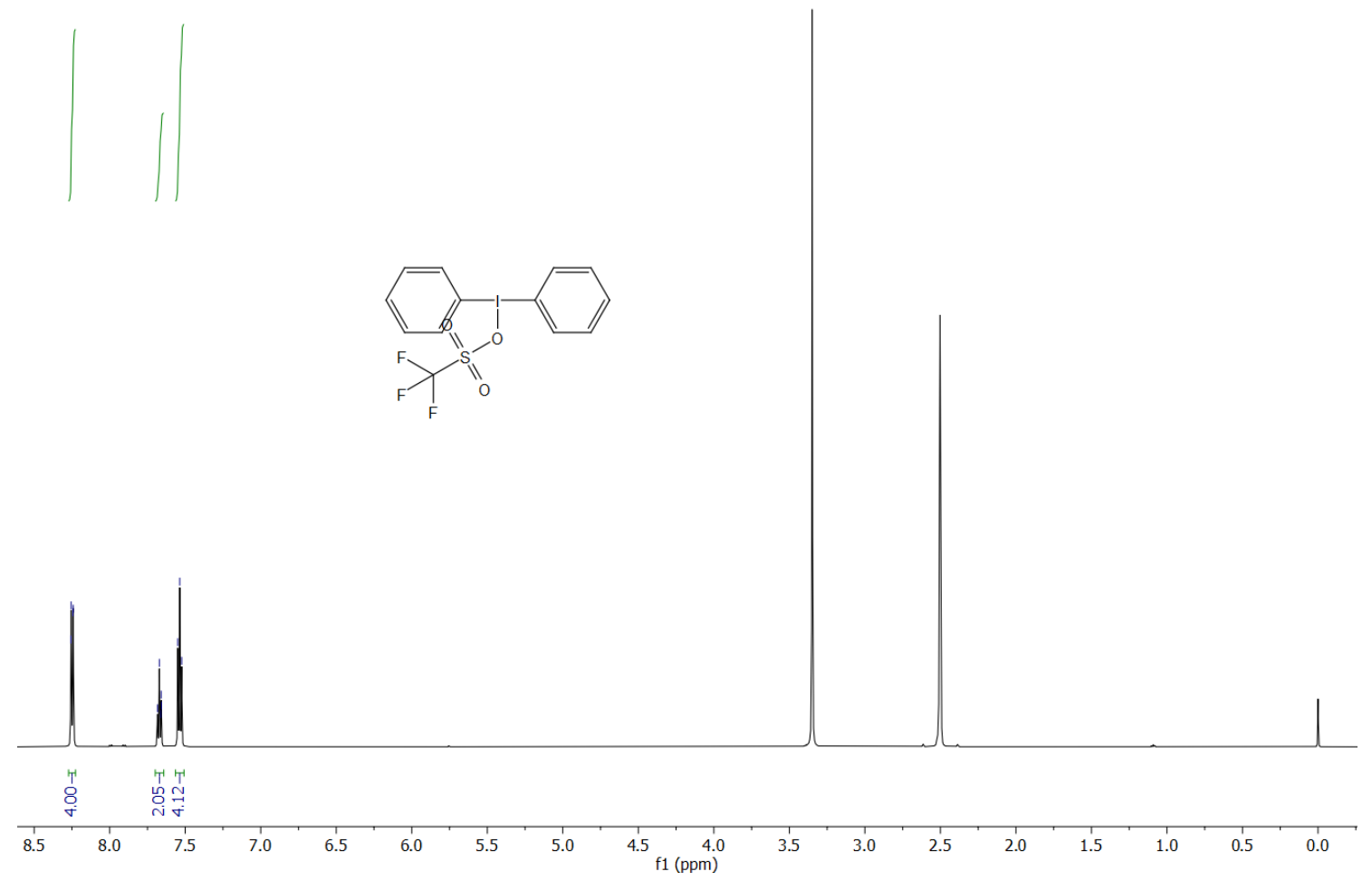

${ }^{1} \mathrm{H}$ NMR spectrum of triphenylsulfonium triflate $\left(600 \mathrm{MHz}, \mathrm{CDCl}_{3}\right)$

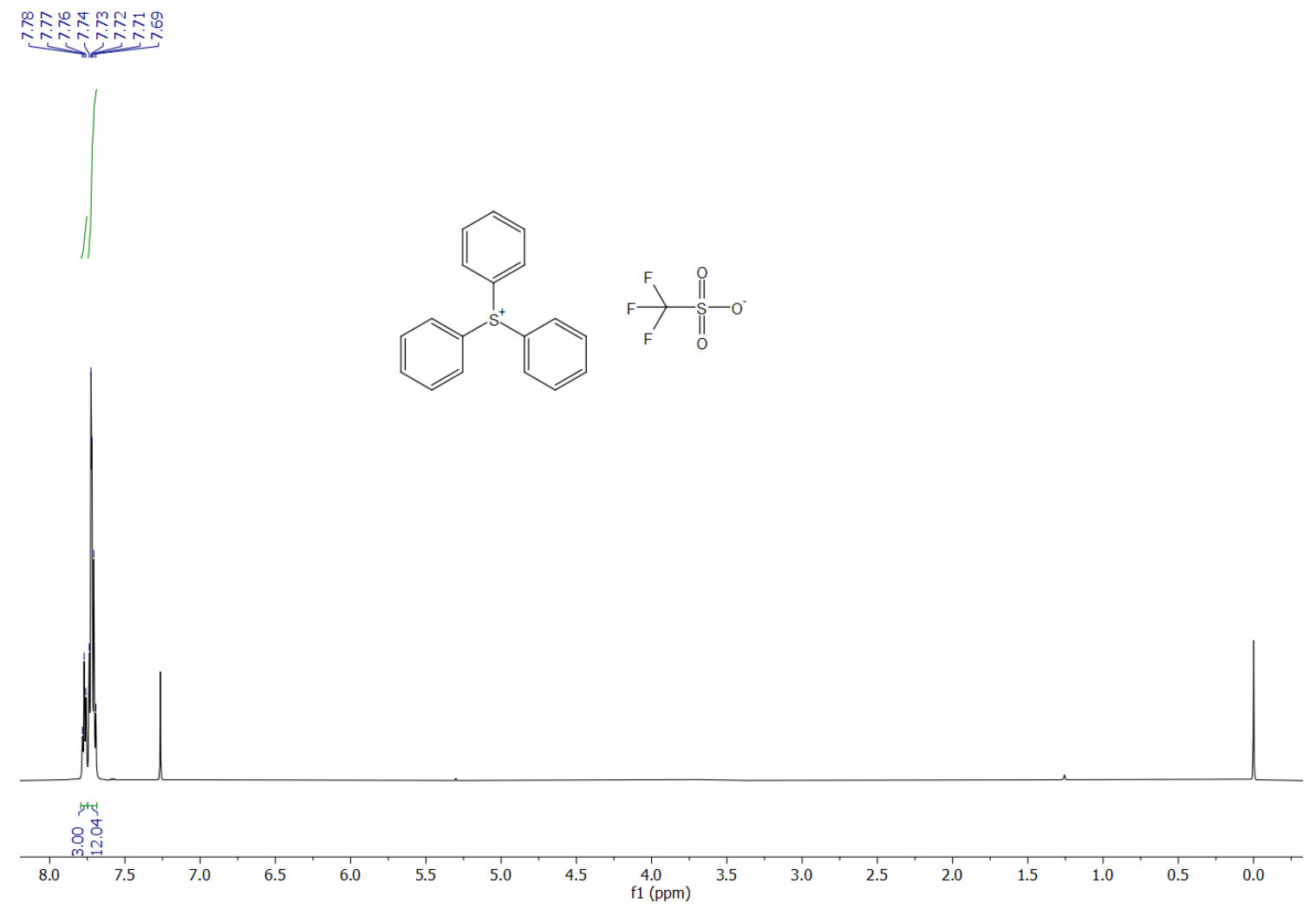


${ }^{1} \mathrm{H}$ NMR spectrum of $N, N$-diphenyl-4-[(trimethylsilyl)ethynyl]aniline (600 $\left.\mathrm{MHz}, \mathrm{CDCl}_{3}\right)$

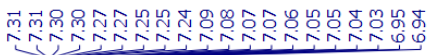
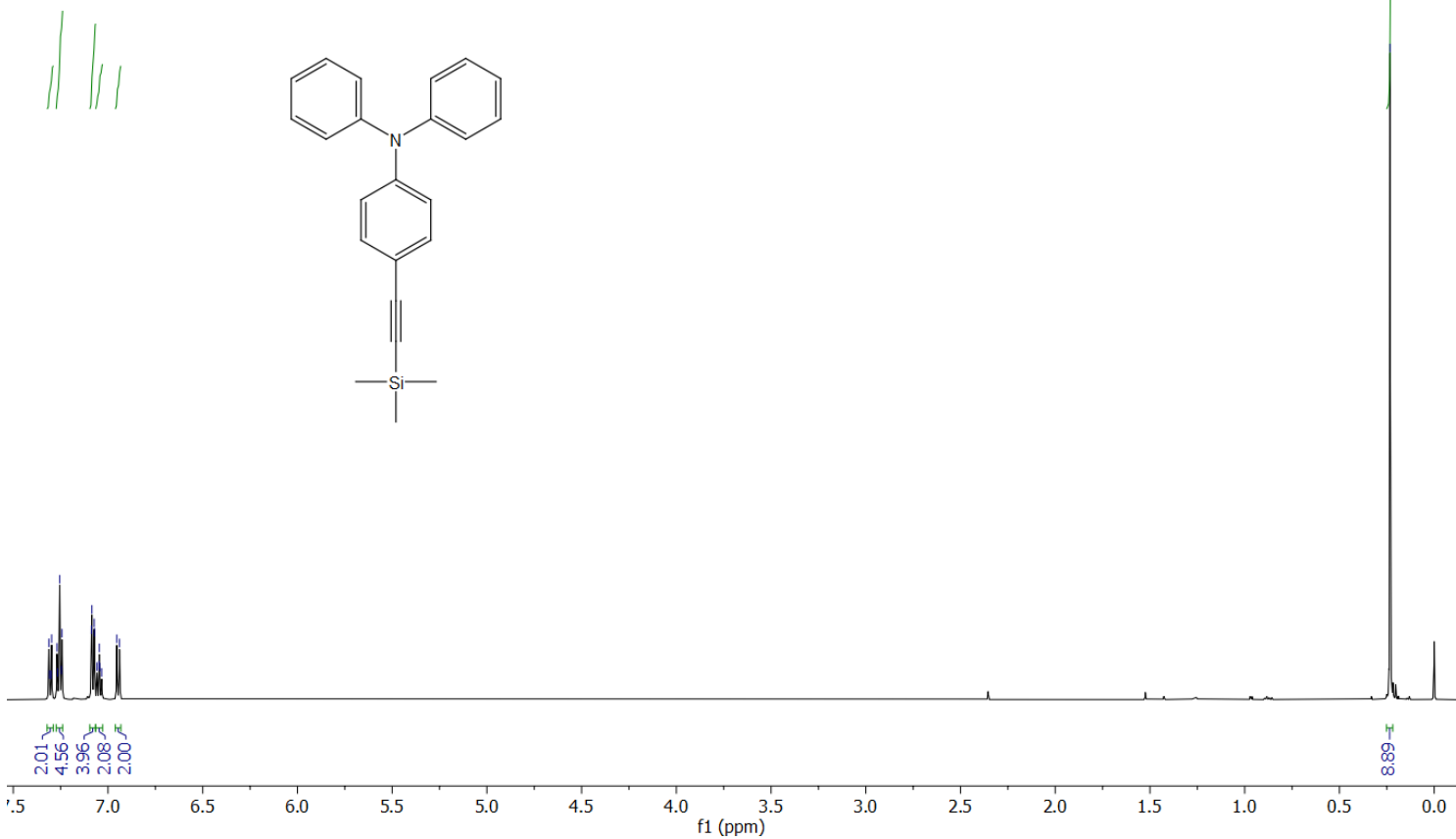

${ }^{1} \mathrm{H}$ NMR spectrum of 4-( $N, N$-diphenylamino)phenylacetylene $\left(600 \mathrm{MHz}, \mathrm{CDCl}_{3}\right)$

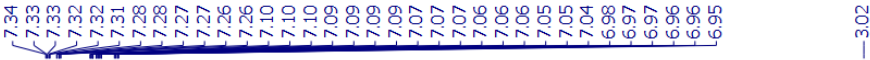
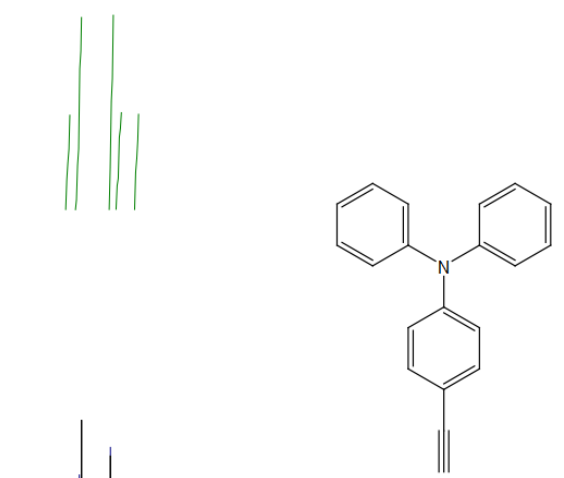

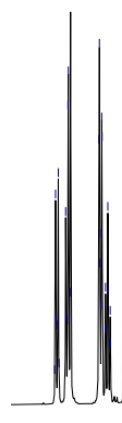
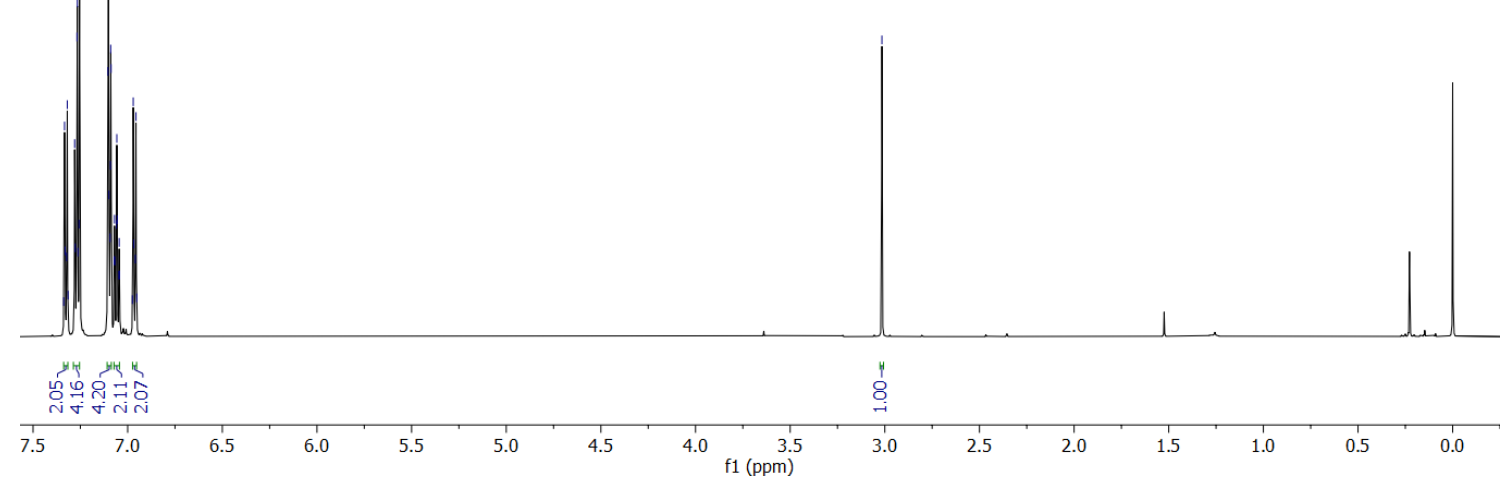
${ }^{1} \mathrm{H}$ NMR spectrum of $\mathrm{N}, \mathrm{N}$-bis(4-methoxyphenyl)aniline (600 $\left.\mathrm{MHz}, \mathrm{CDCl}_{3}\right)$

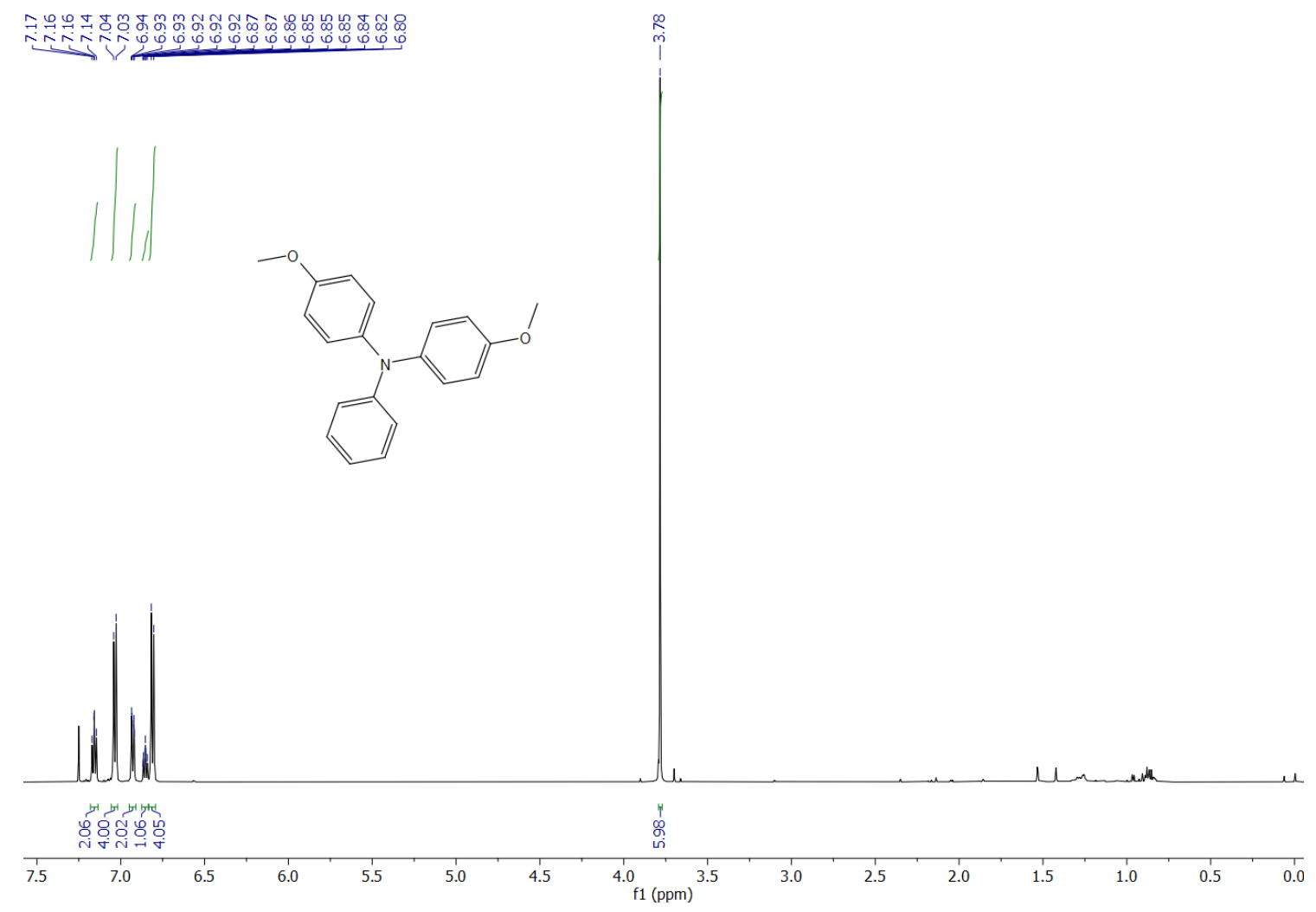

${ }^{1} \mathrm{H}$ NMR spectrum of 4-iodo- $N, N$-bis(4-methoxyphenyl)aniline (600 $\left.\mathrm{MHz}, \mathrm{CDCl}_{3}\right)$

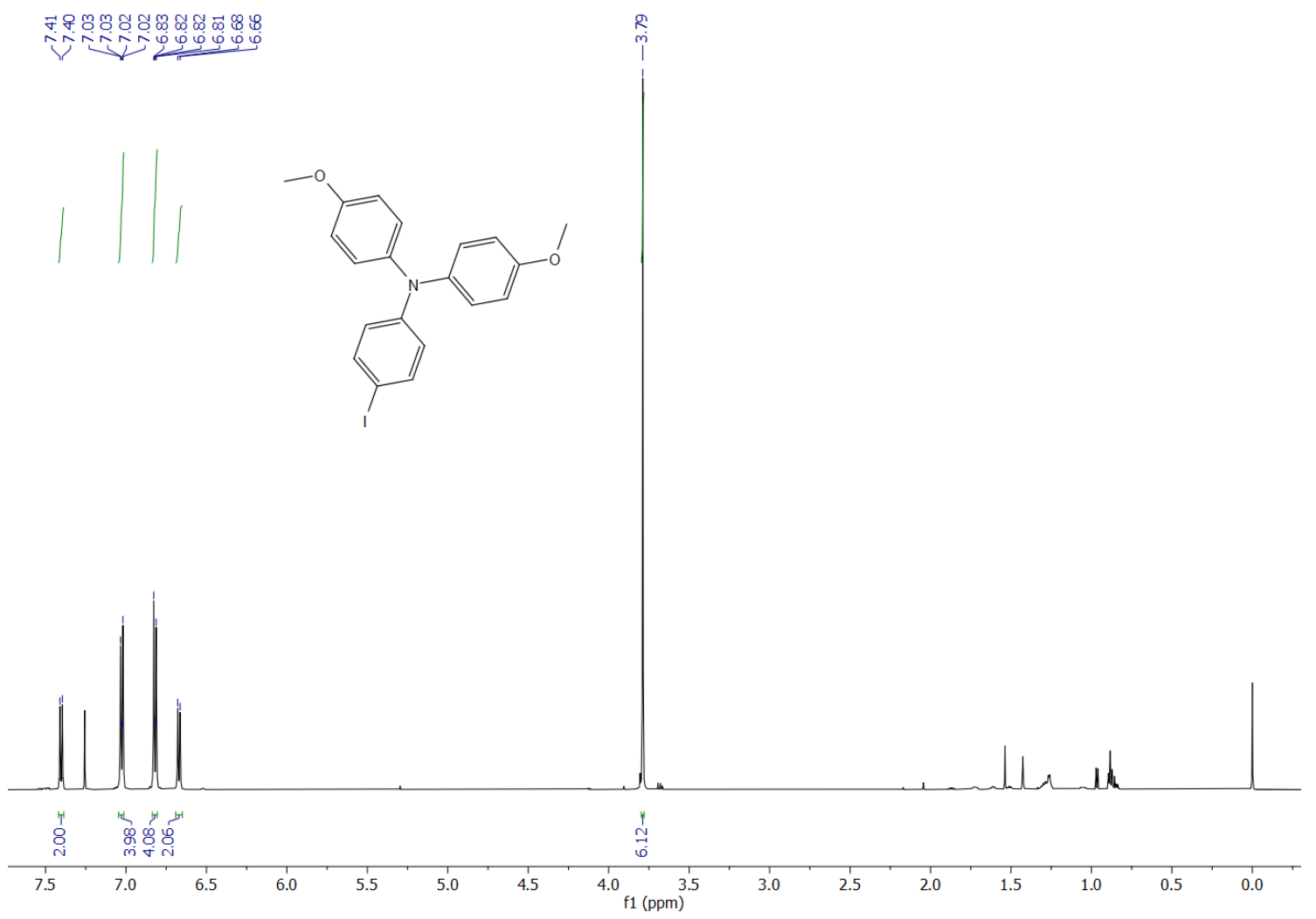


${ }^{1}$ H NMR spectrum of $N, N$-bis(4-methoxyphenyl)-4-[(trimethylsilyl)ethynyl]aniline (600 $\mathrm{MHz} \mathrm{CDCl}_{3}$ )

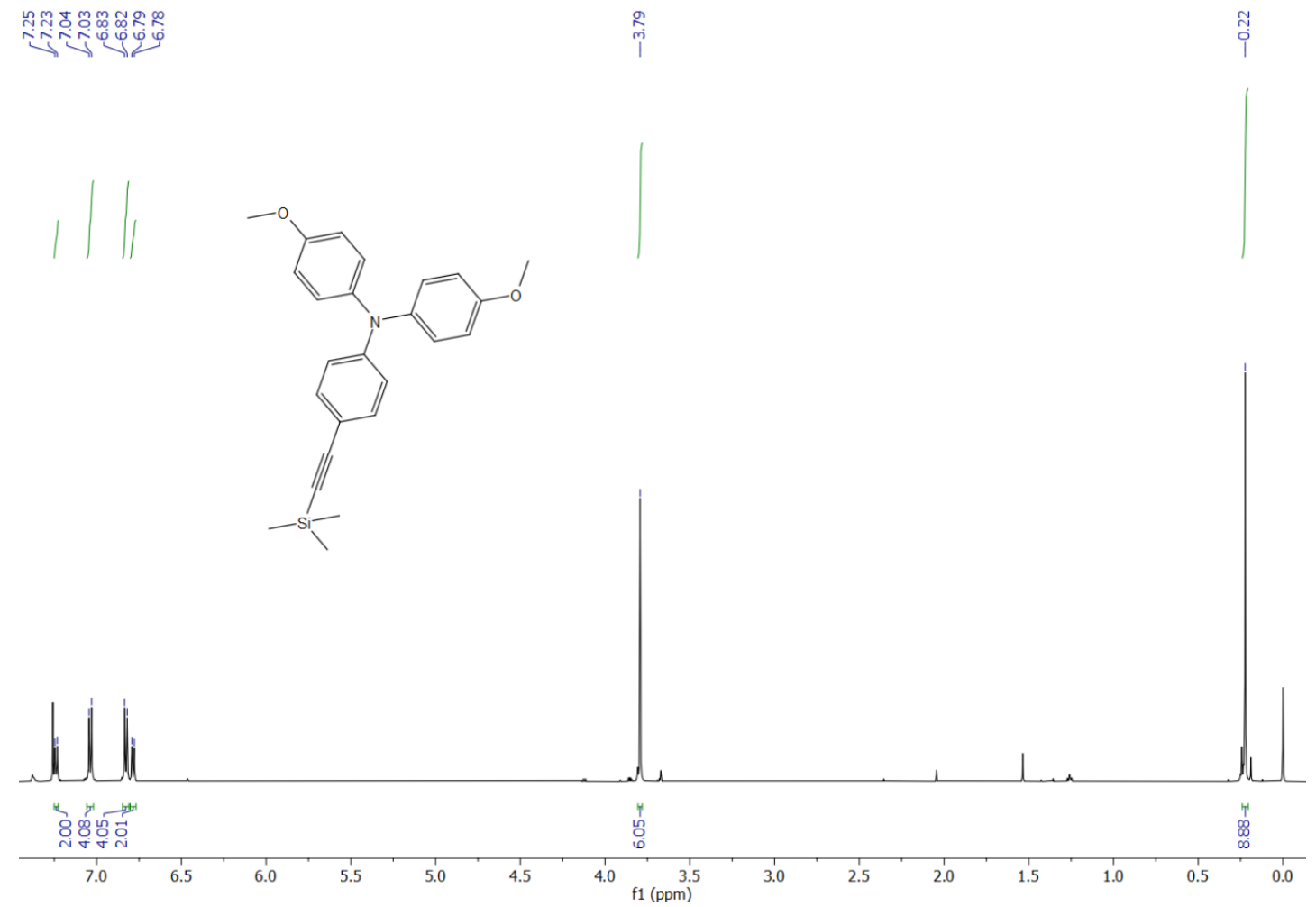

${ }^{1}$ H NMR spectrum of 4-[N,N-bis(4-methoxyphenyl)amino]phenylacetylene (600 MHz, $\mathrm{CDCl}_{3}$ )

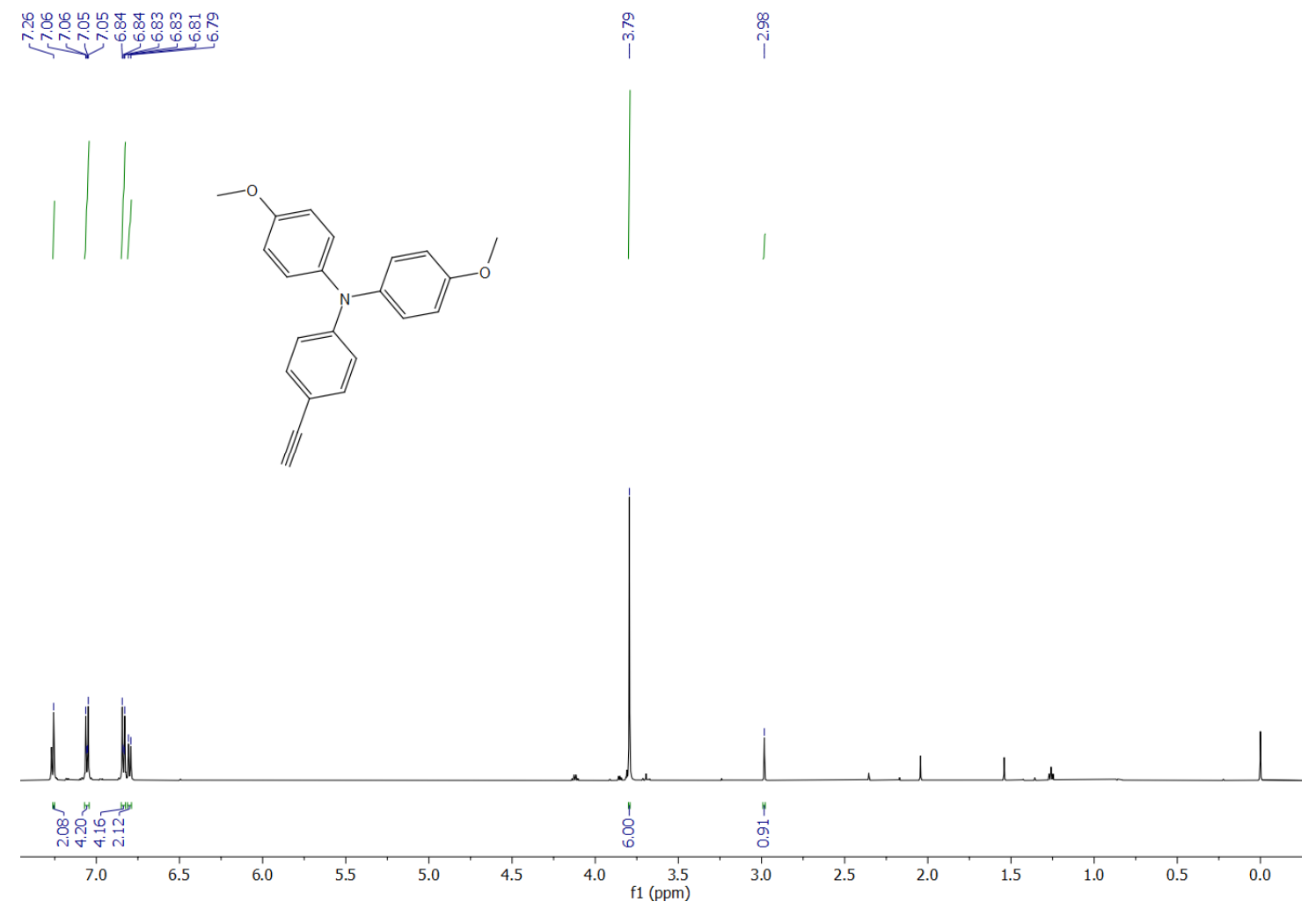


${ }^{1} \mathrm{H}$ NMR spectrum of 4-( $N, N$-diphenylamino)phenylboronic acid (600 $\left.\mathrm{MHz}, \mathrm{CDCl}_{3}\right)$

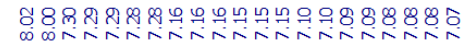

ب
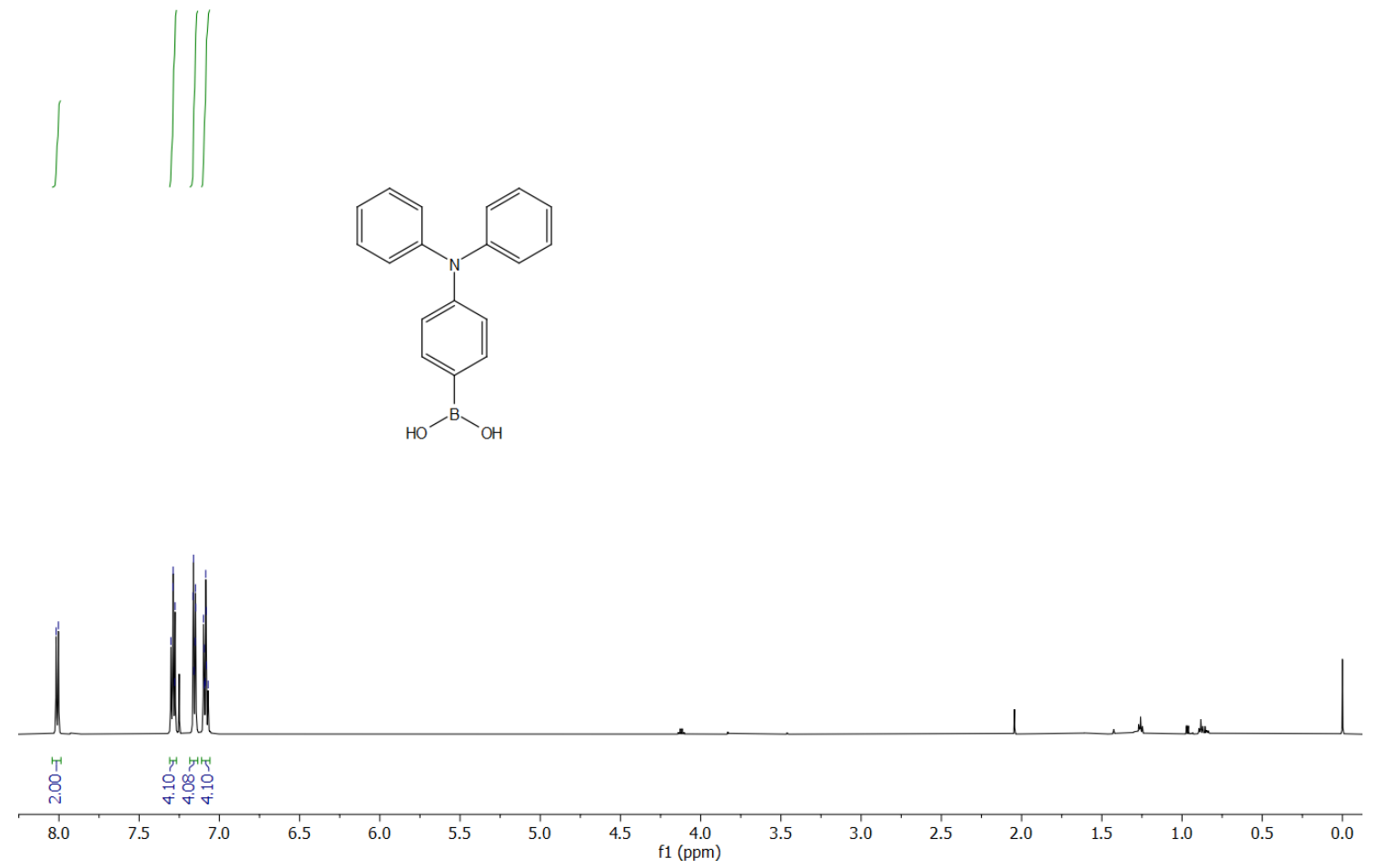

${ }^{1} \mathrm{H}$ NMR spectrum of 4-[N,N-bis(4-methoxyphenyl)amino]phenylboronic acid $\left(600 \mathrm{MHz}, \mathrm{CDCl}_{3}\right)$

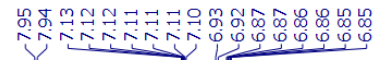
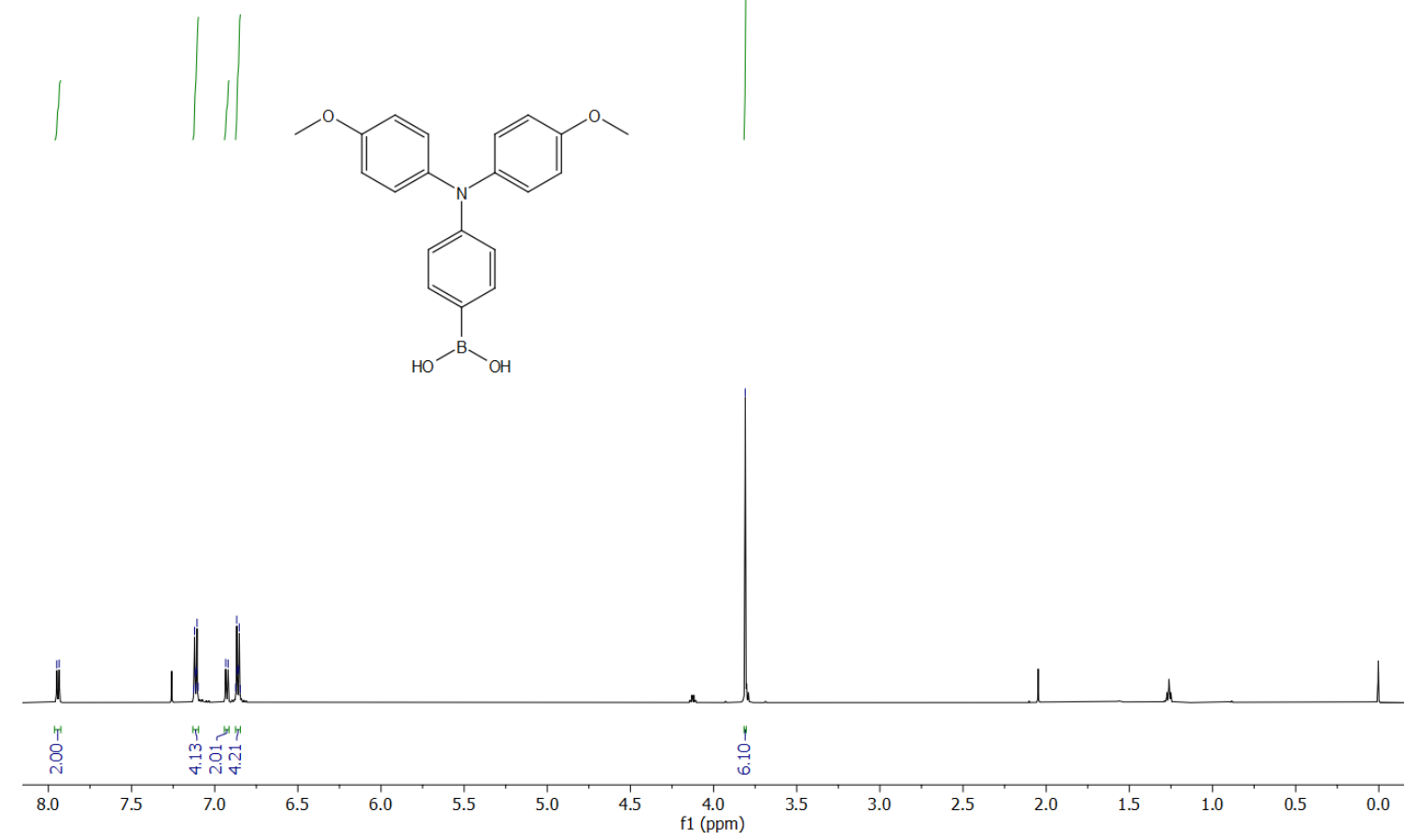
${ }^{1}$ H NMR spectrum of btz-I (600 MHz, DMSO-d $)_{\text {) }}$

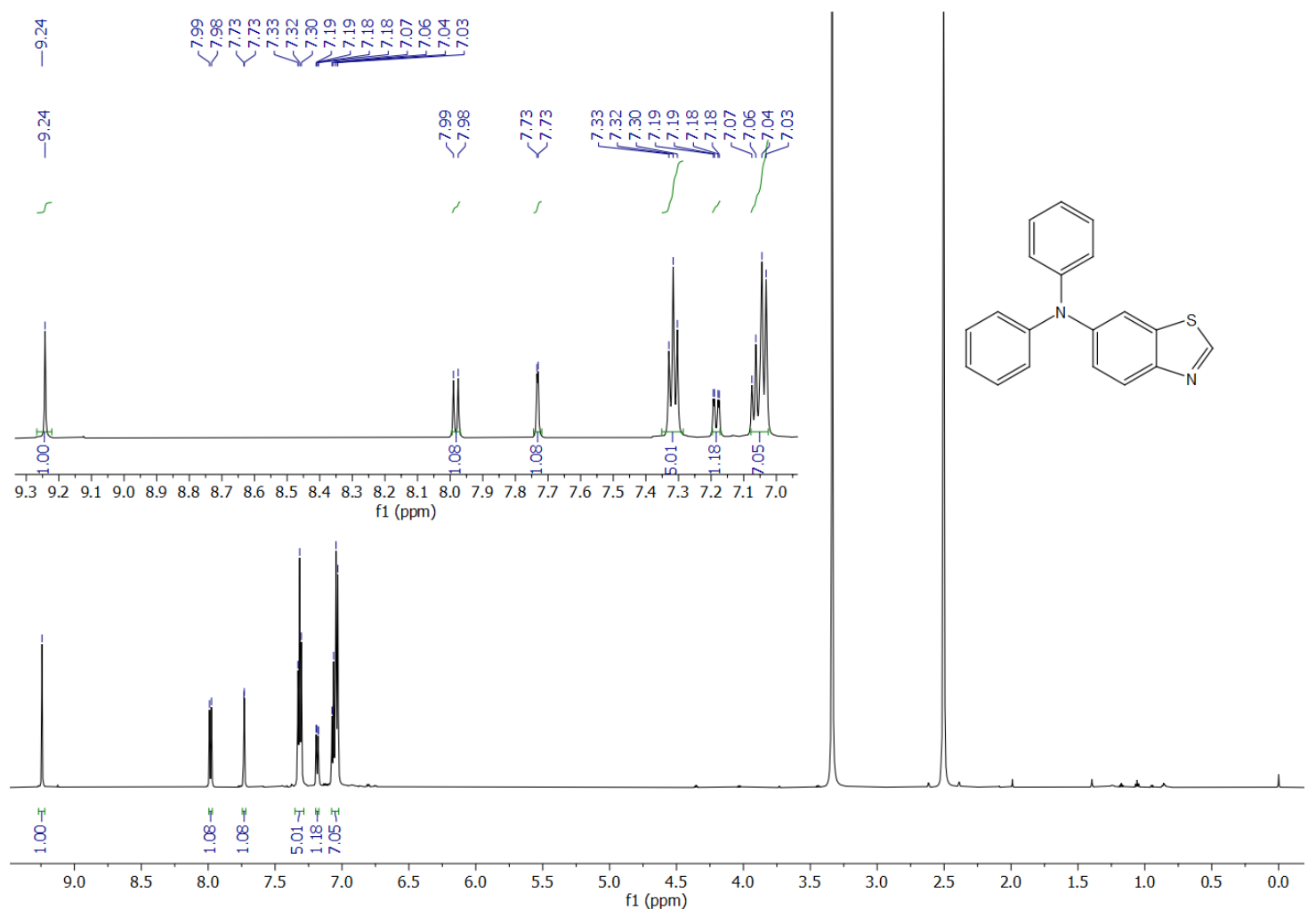

${ }^{13} \mathrm{C}$ NMR spectrum of btz-I (151 MHz, DMSO-d $\left.d_{6}\right)$

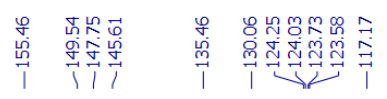

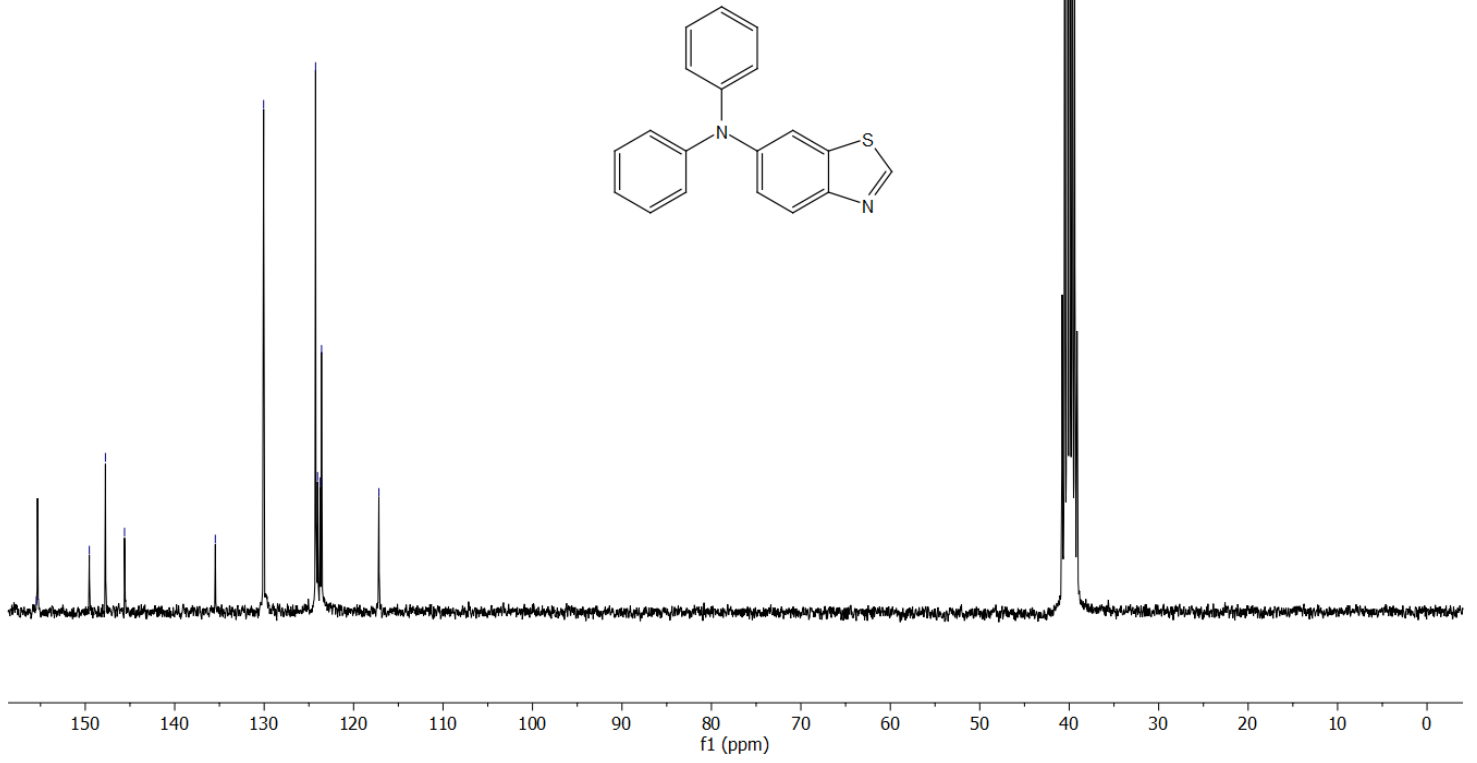

S38 | Supporting Information 
${ }^{1} \mathrm{H}$ NMR spectrum of btz-II (600 $\left.\mathrm{MHz}, \mathrm{CDCl}_{3}\right)$

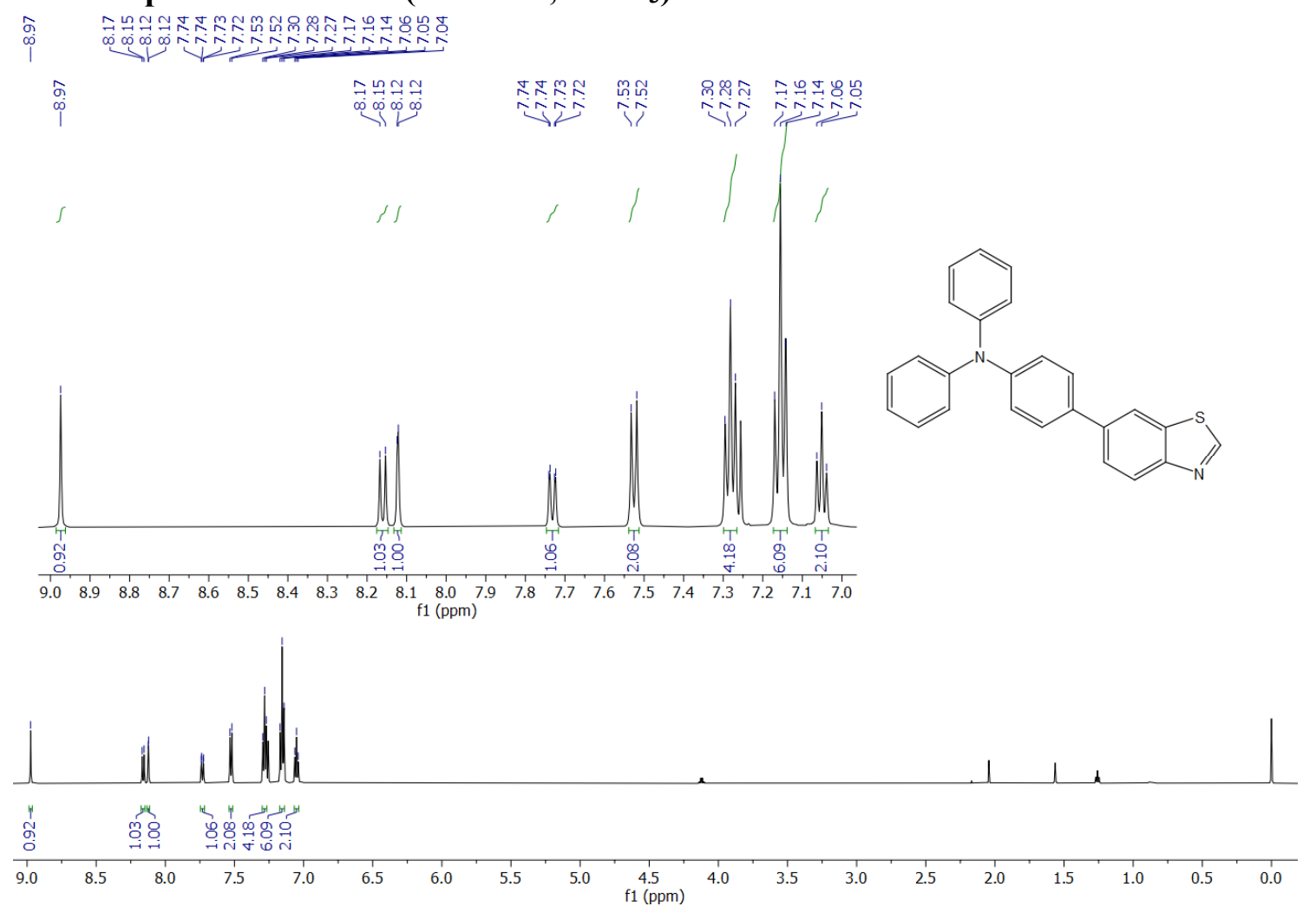

${ }^{13} \mathrm{C}$ NMR spectrum of btz-II (151 MHz, $\left.\mathrm{CDCl}_{3}\right)$

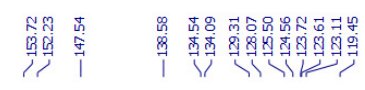

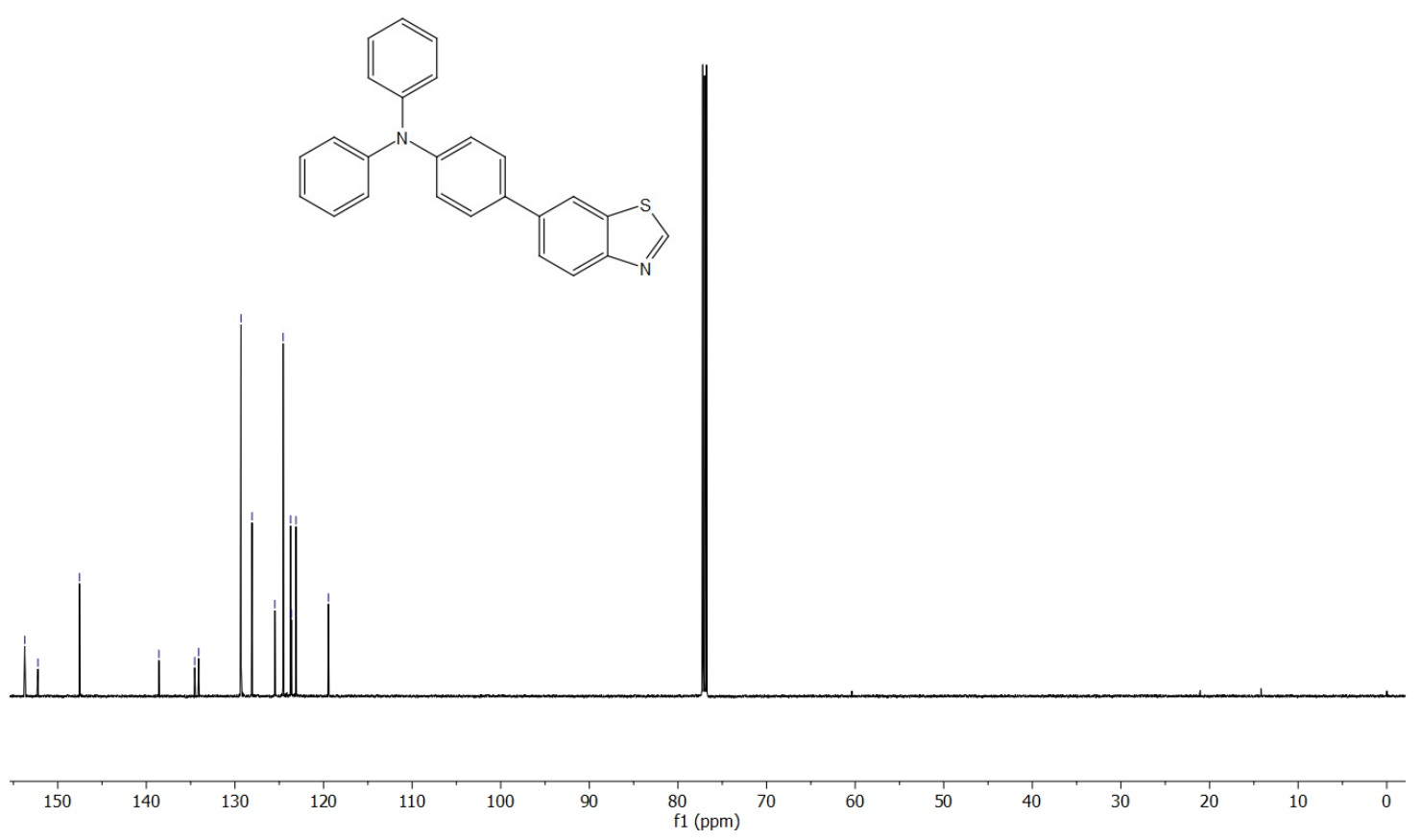

S39 | Supporting Information 
${ }^{1} \mathrm{H}$ NMR spectrum of btz-III (600 $\left.\mathrm{MHz}, \mathrm{CDCl}_{3}\right)$

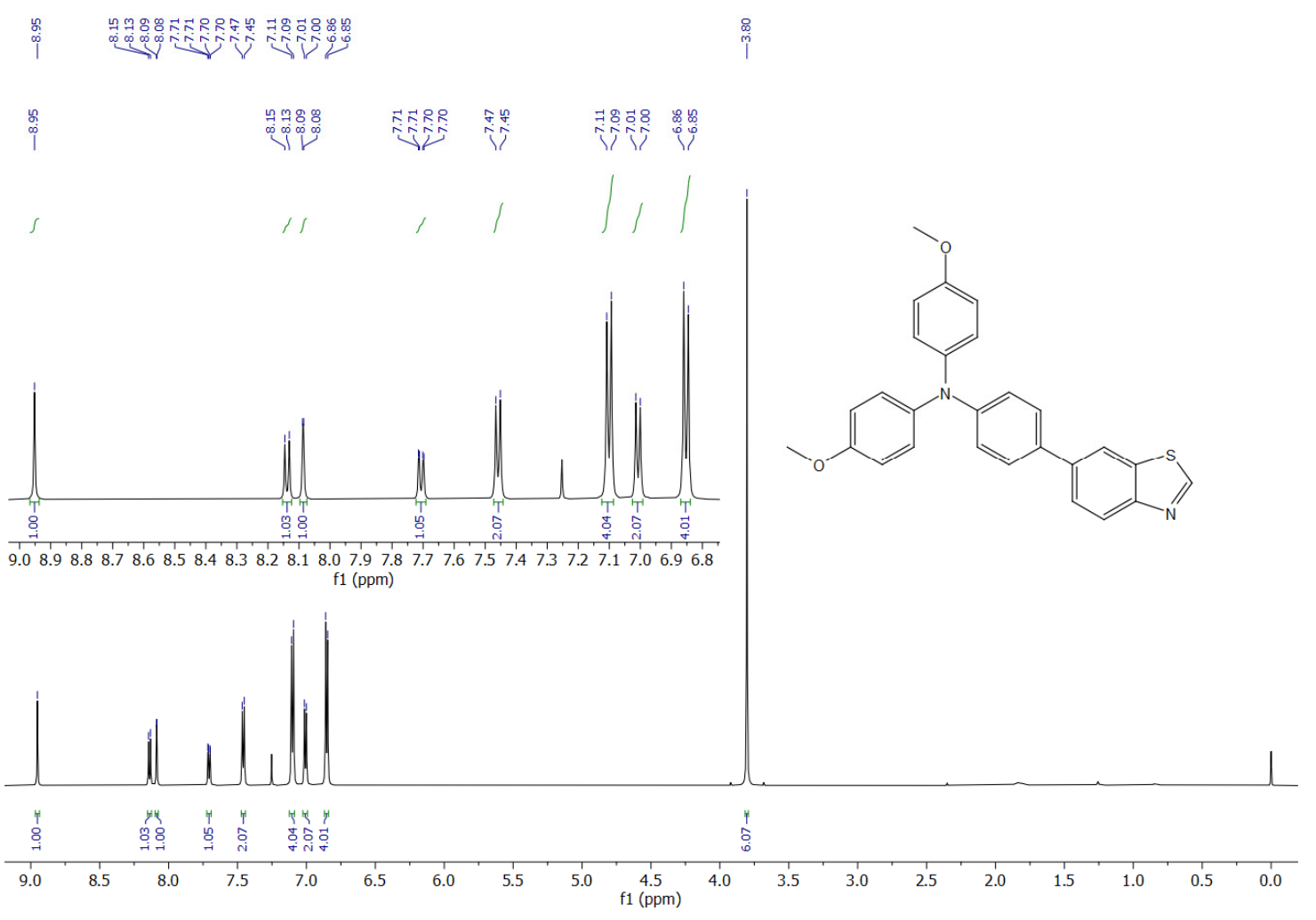

${ }^{13} \mathrm{C}$ NMR spectrum of btz-III (151 $\left.\mathrm{MHz}, \mathrm{CDCl}_{3}\right)$

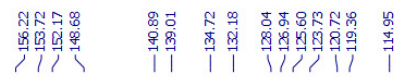

产
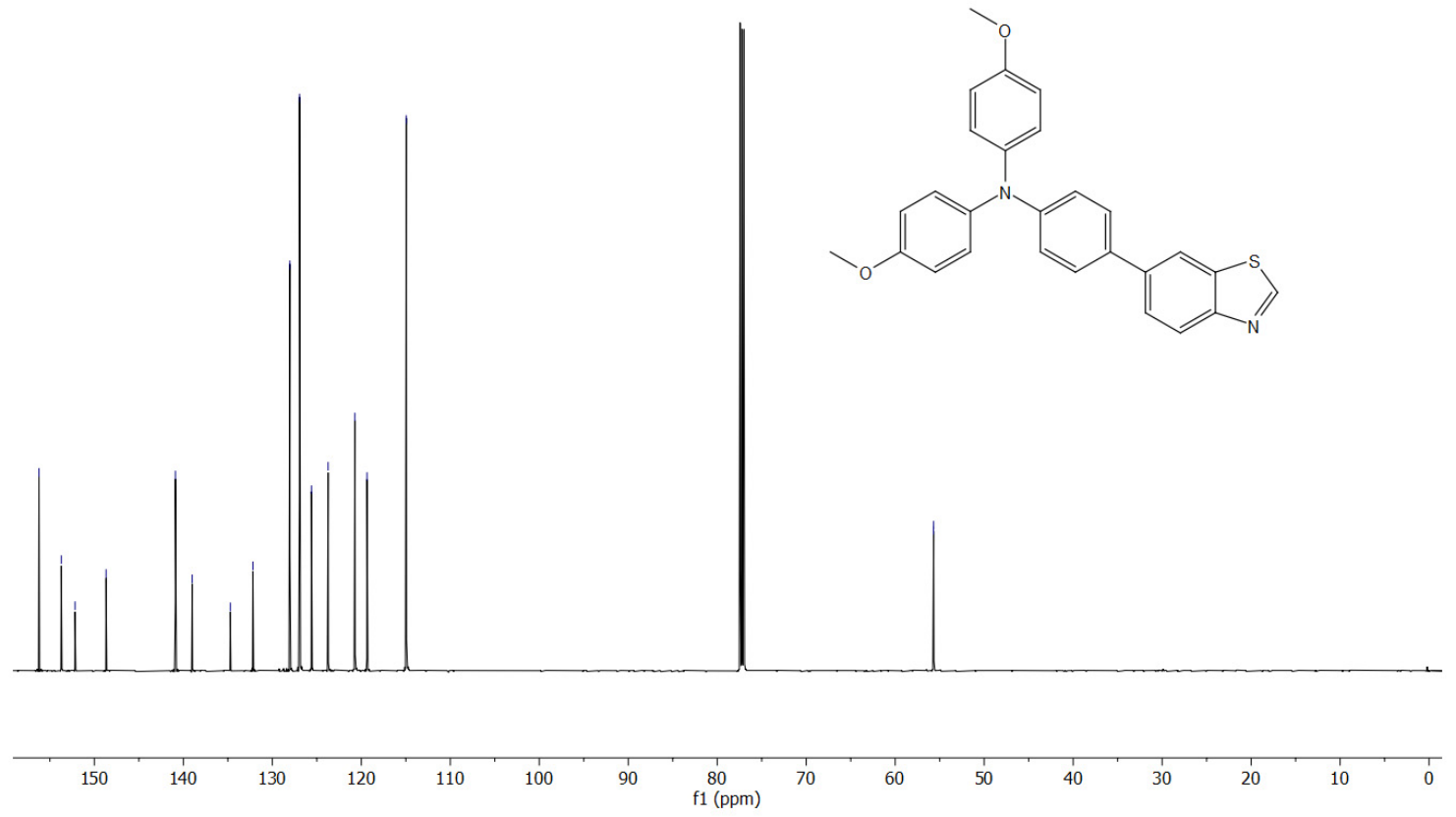

S40 | Supporting Information 
${ }^{1} \mathrm{H}$ NMR spectrum of btz-IV (600 MHz, $\left.\mathrm{CDCl}_{3}\right)$

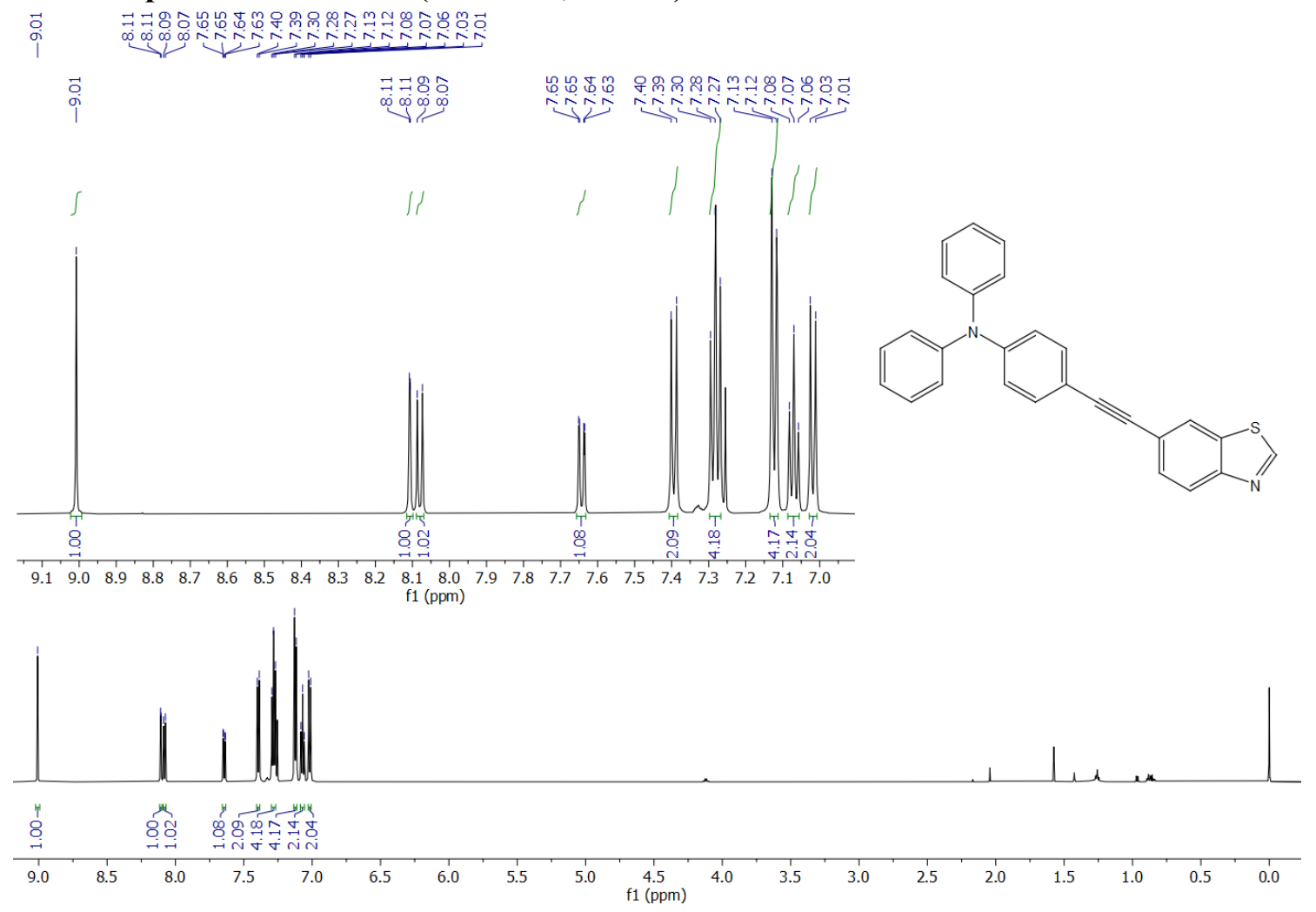

${ }^{13} \mathrm{C}$ NMR spectrum of btz-IV (151 MHz, $\left.\mathrm{CDCl}_{3}\right)$

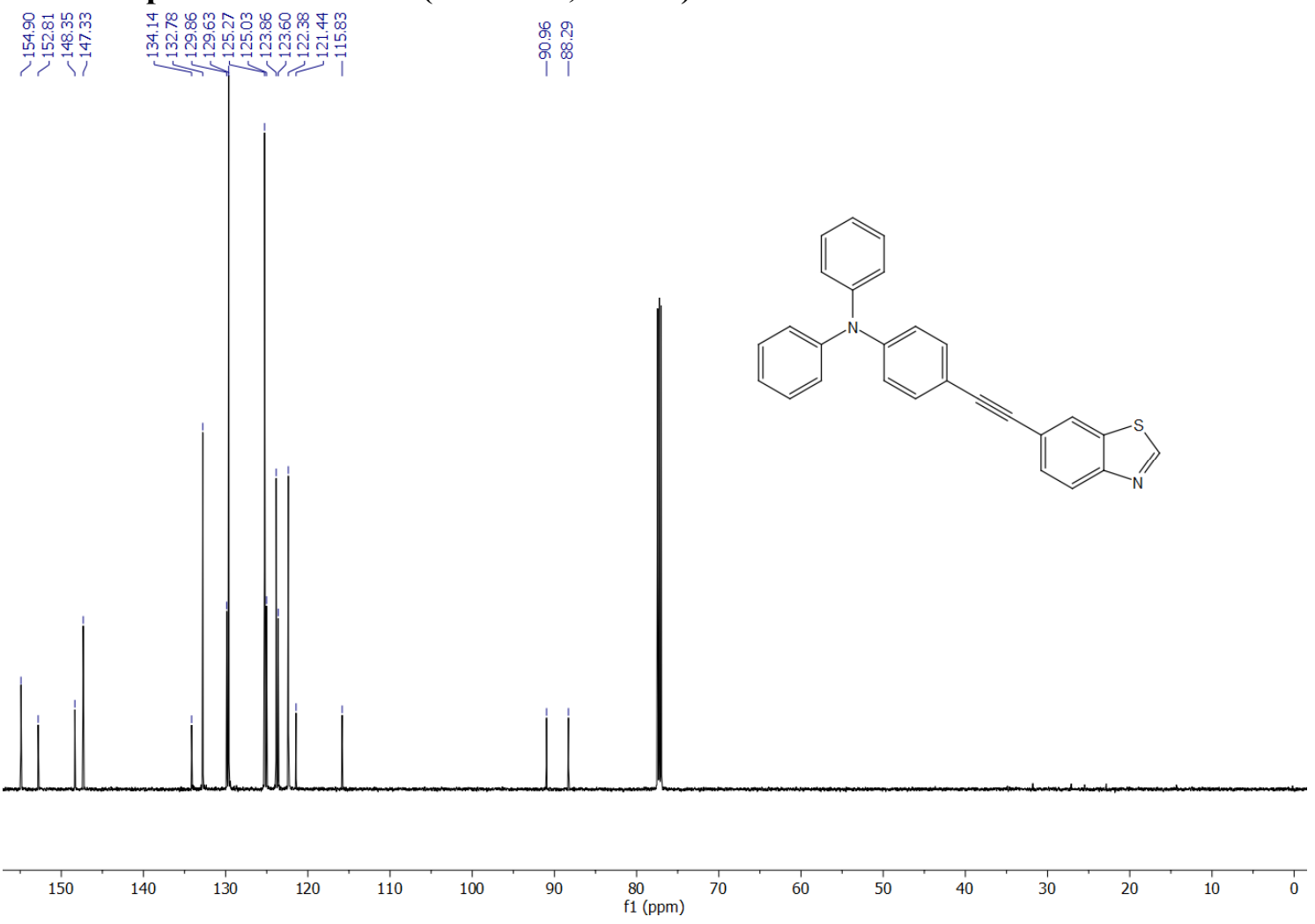

S41 | Supporting Information 
${ }^{1} \mathrm{H}$ NMR spectrum of btz-V (600 $\left.\mathrm{MHz}, \mathrm{CDCl}_{3}\right)$

i

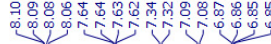

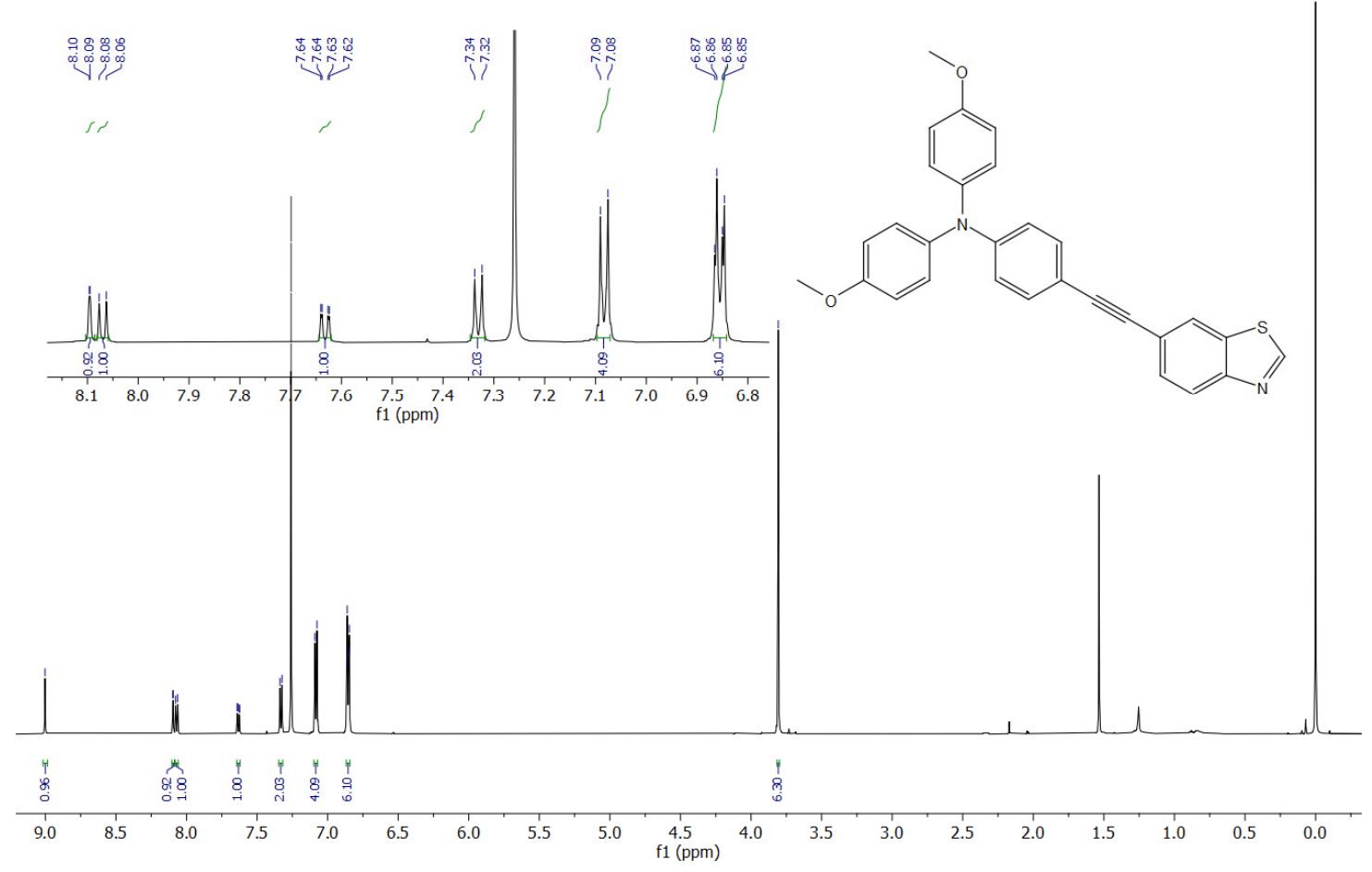

${ }^{13} \mathrm{C}$ NMR spectrum of btz-V (151 $\left.\mathrm{MHz}, \mathrm{CDCl}_{3}\right)$
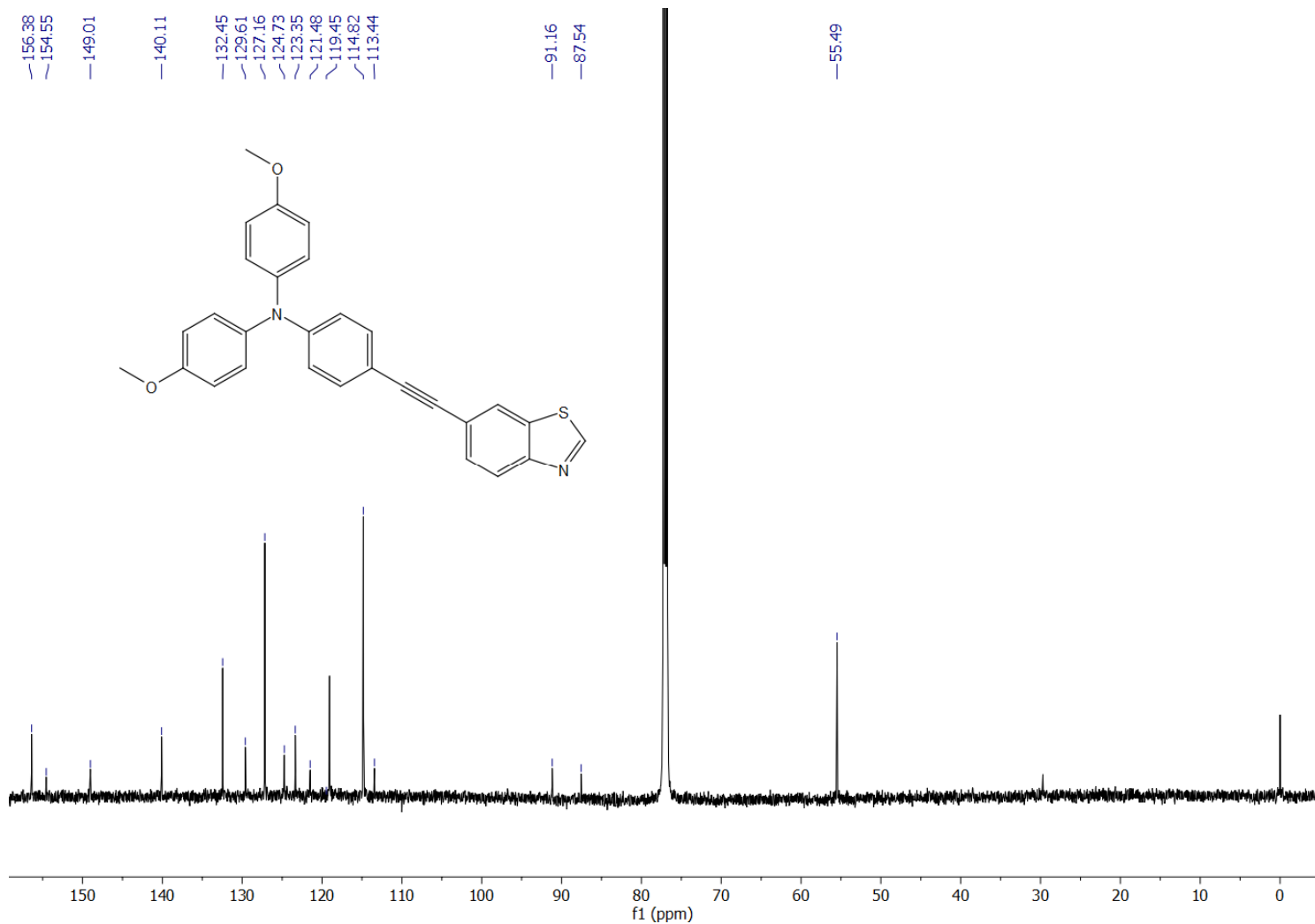
${ }^{1} \mathrm{H}$ NMR spectrum of btz-VI (600 MHz, $\left.\mathrm{CDCl}_{3}\right)$
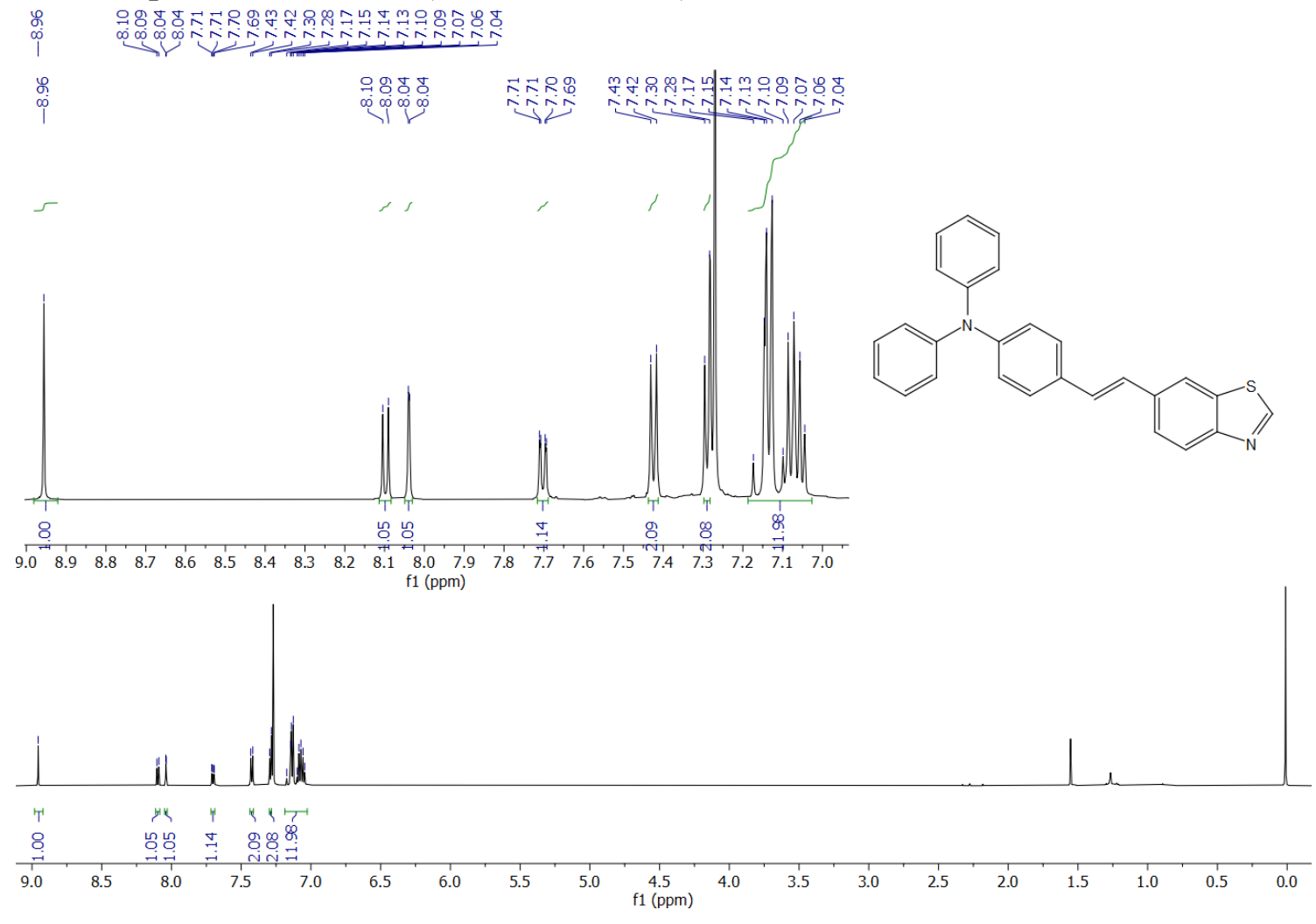

${ }^{13} \mathrm{C}$ NMR spectrum of btz-VI (151 MHz, $\left.\mathrm{CDCl}_{3}\right)$
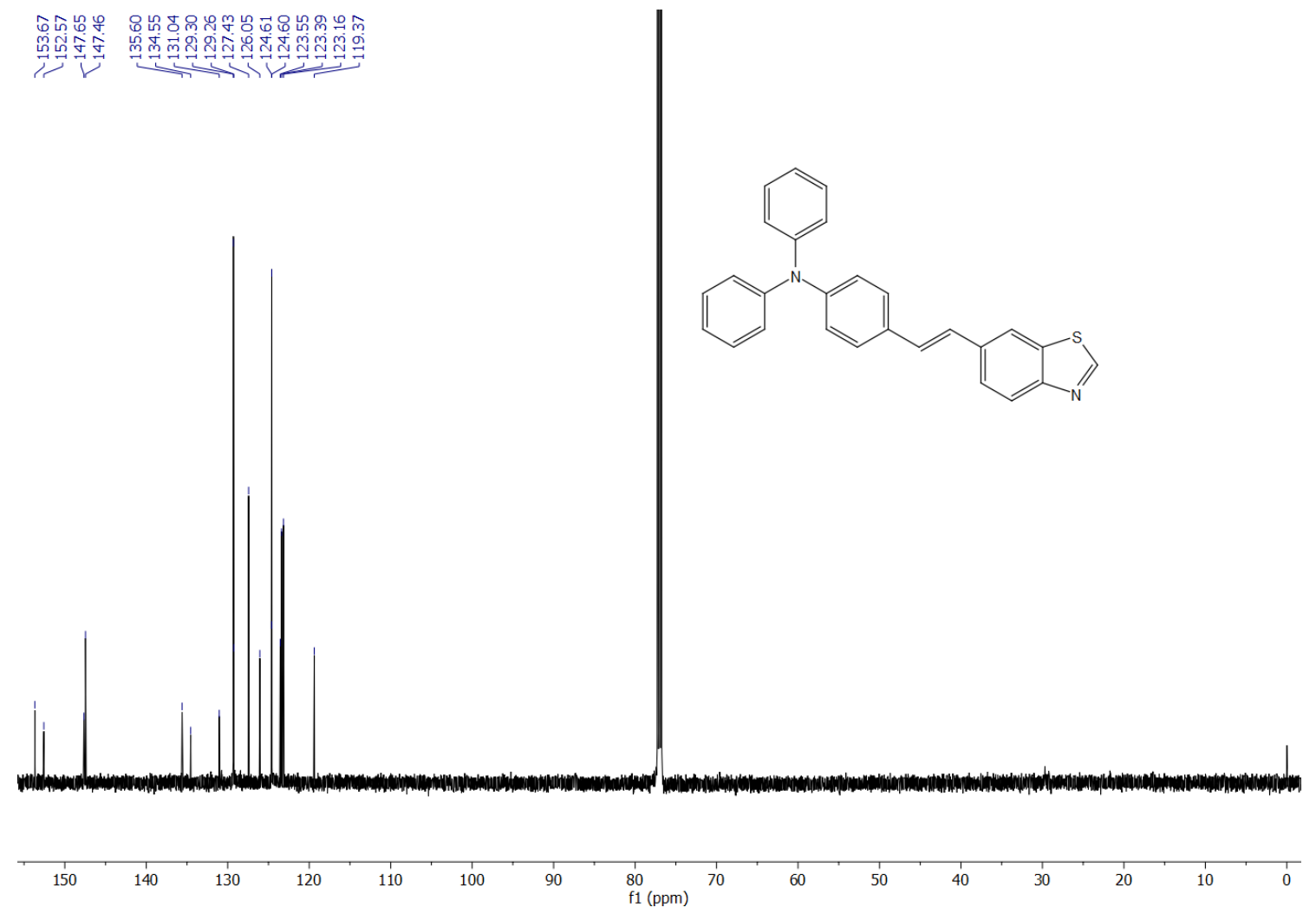

S43 | Supporting Information 
${ }^{1} \mathrm{H}$ NMR spectrum of 2,2'-bibenzothiazole (600 $\left.\mathrm{MHz}, \mathrm{CDCl}_{3}\right)$
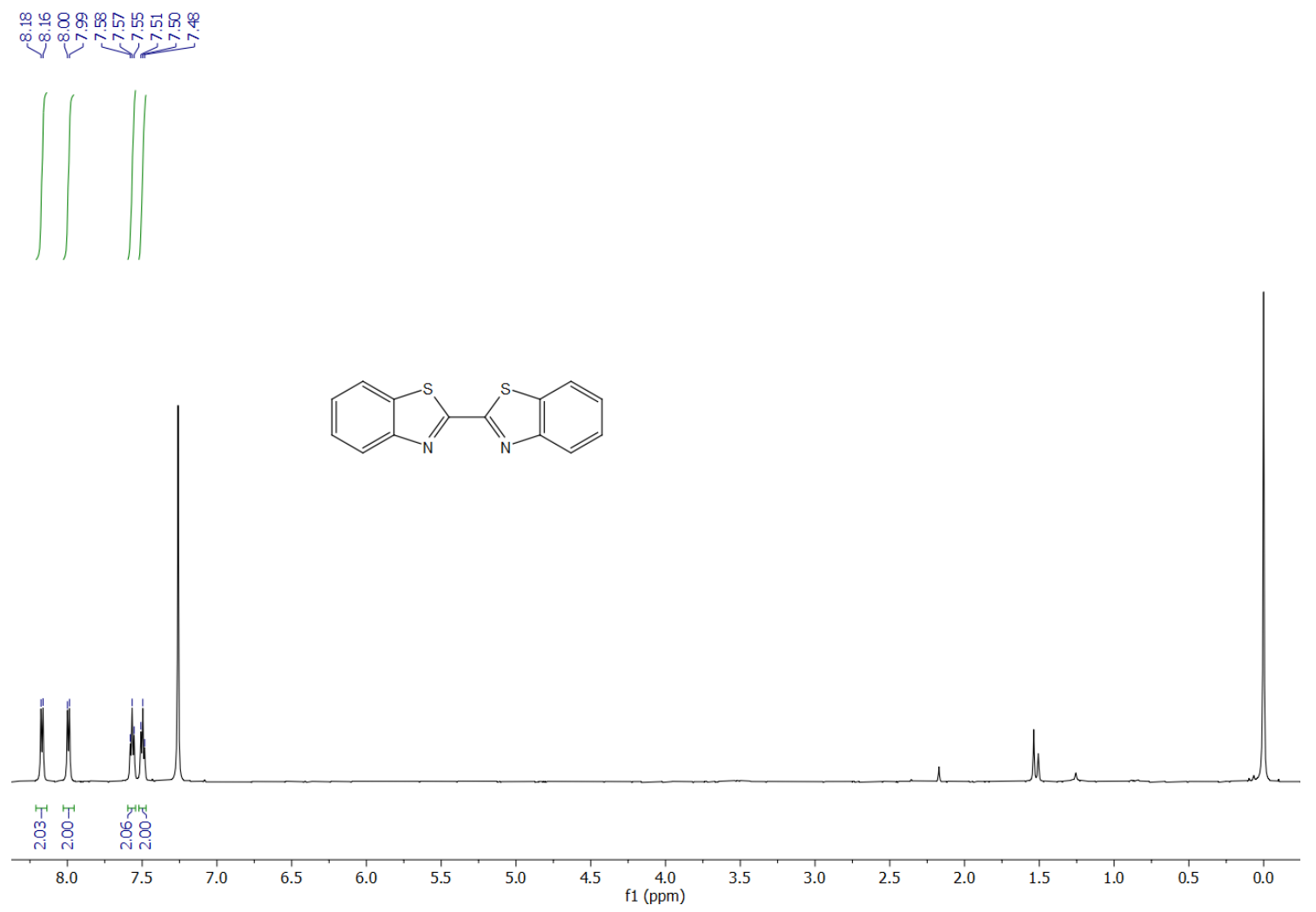

${ }^{13} \mathrm{C}$ NMR spectrum of 2,2'-bibenzothiazole (151 $\mathrm{MHz}, \mathrm{CDCl}_{3}$ )
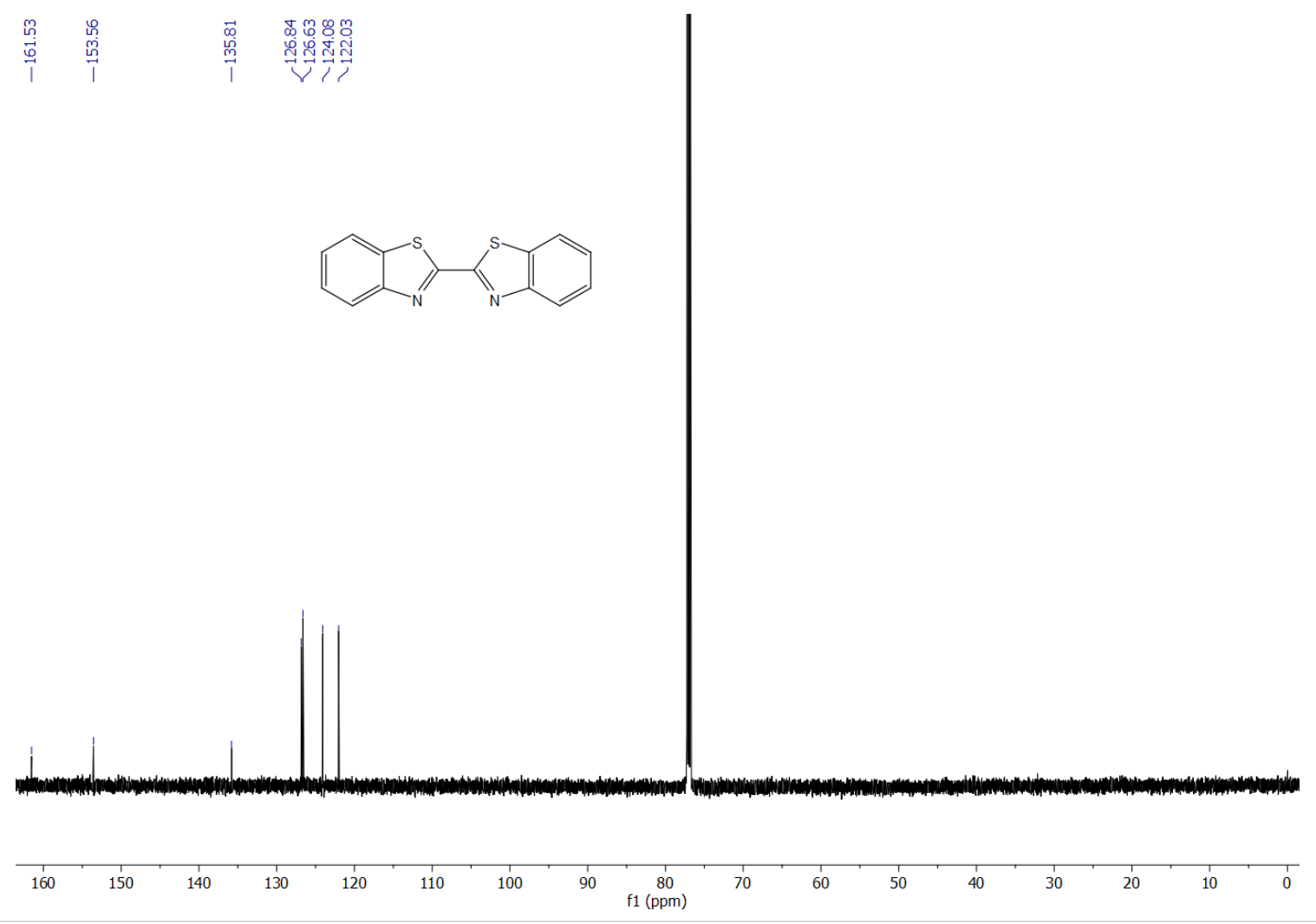

S44 | Supporting Information 
${ }^{1} \mathrm{H}$ NMR spectrum of bisbtz-I (600 MHz, $\left.\mathrm{CDCl}_{3}\right)$

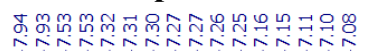
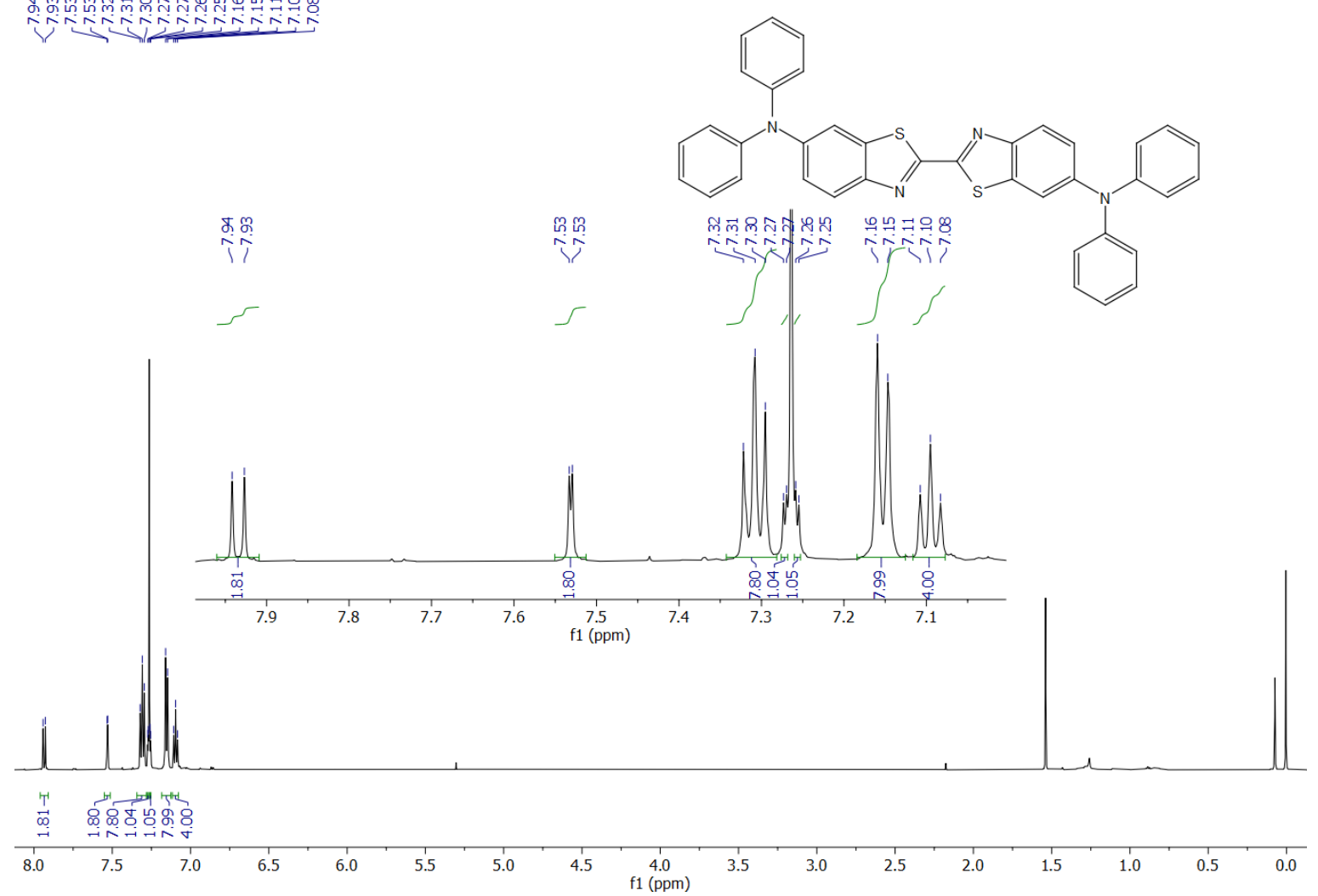

${ }^{13} \mathrm{C}$ NMR spectrum of bisbtz-I (151 MHz, $\mathrm{CDCl}_{3}$ )
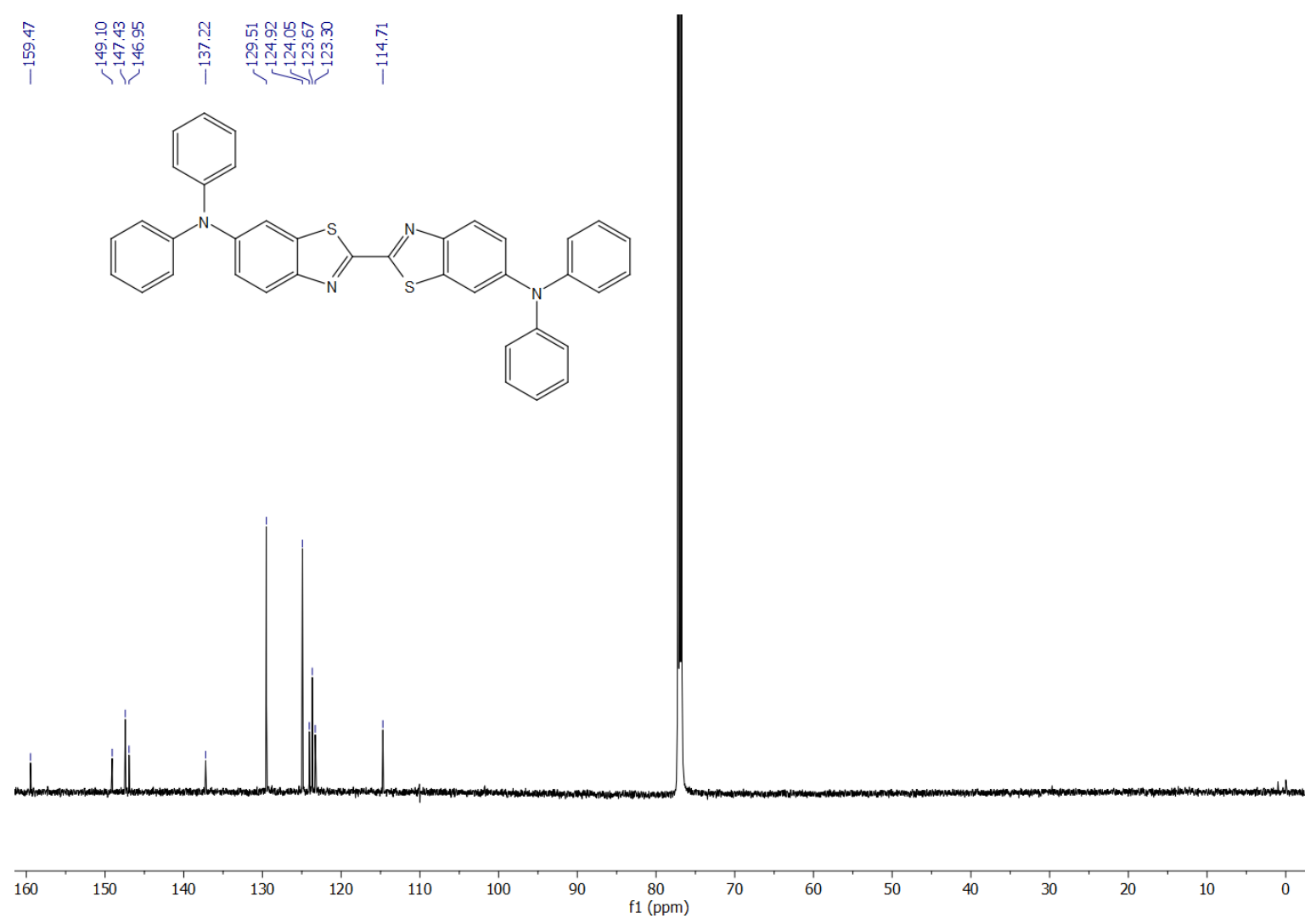

S45 | Supporting Information 
${ }^{15} \mathrm{~N}$ NMR spectrum of bisbtz-I (61 $\left.\mathrm{MHz}, \mathrm{CDCl}_{3}\right)$

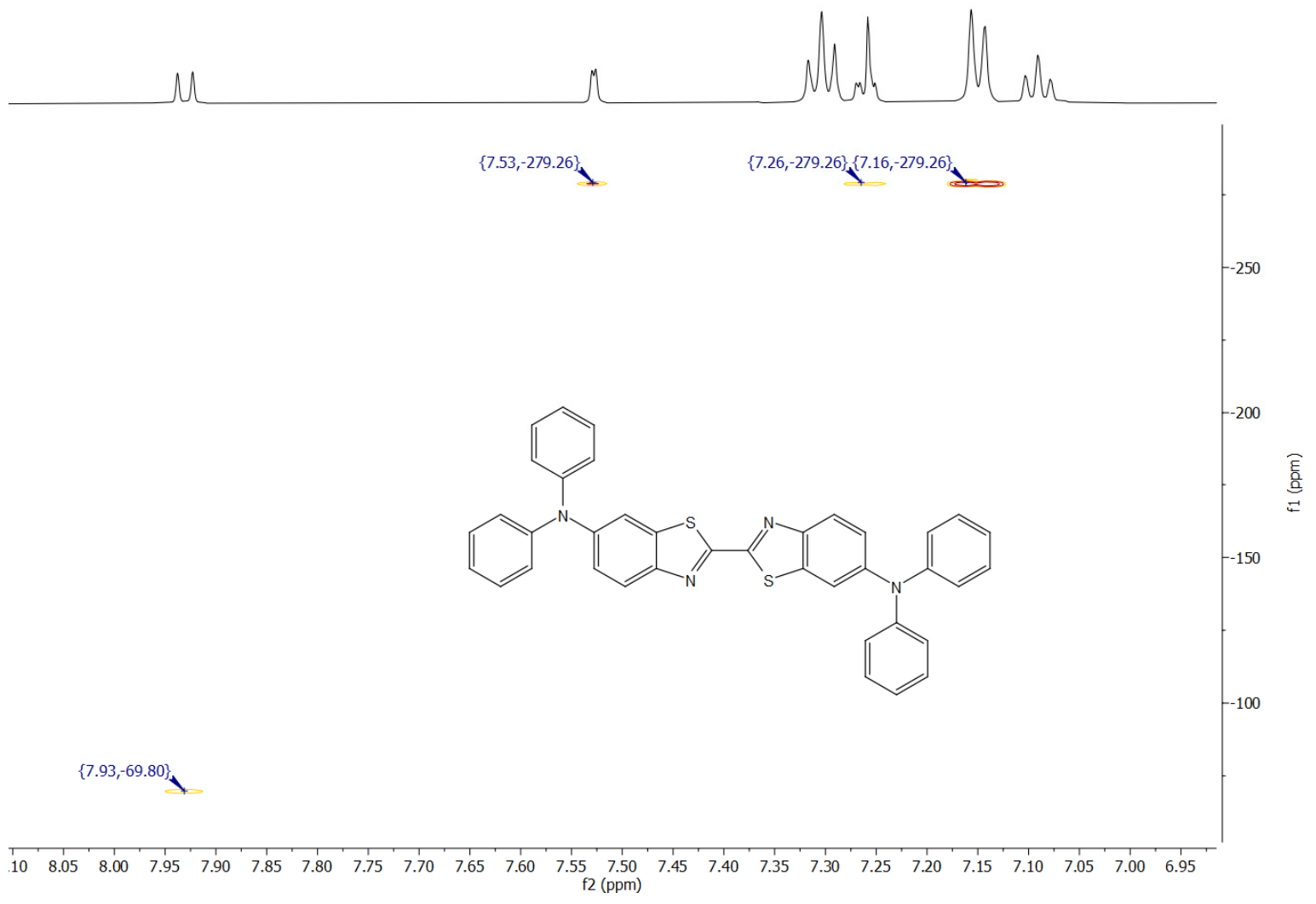

S46 | Supporting Information 
${ }^{1} \mathrm{H}$ NMR spectrum of bisbtz-II (600 $\left.\mathrm{MHz}, \mathrm{CDCl}_{3}\right)$

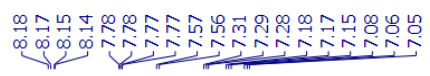

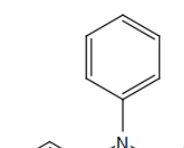<smiles>CCNc1ccccc1</smiles><smiles>c1ccc(Cc2ccc(-c3ccc4nc(-c5nc6ccc(-c7ccccc7)cc6s5)sc4c3)cc2)cc1</smiles>

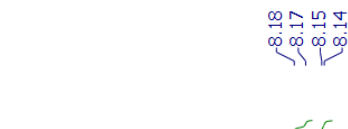

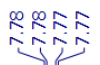

$\stackrel{\substack{n \\ \text { i 昌 }}}{1}$

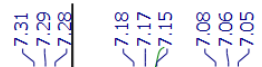
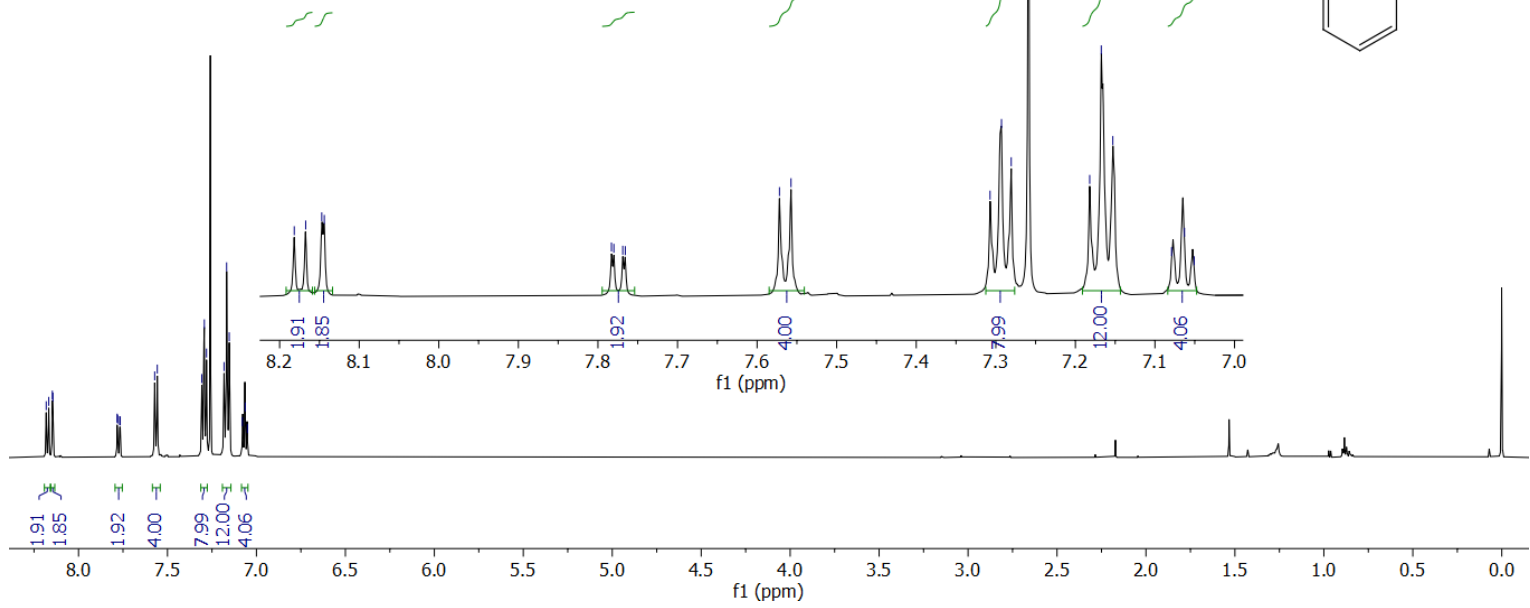

${ }^{13} \mathrm{C}$ NMR spectrum of bisbtz-II (151 $\left.\mathrm{MHz}, \mathrm{CDCl}_{3}\right)$

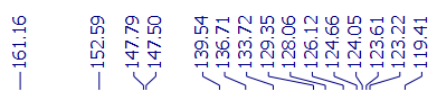

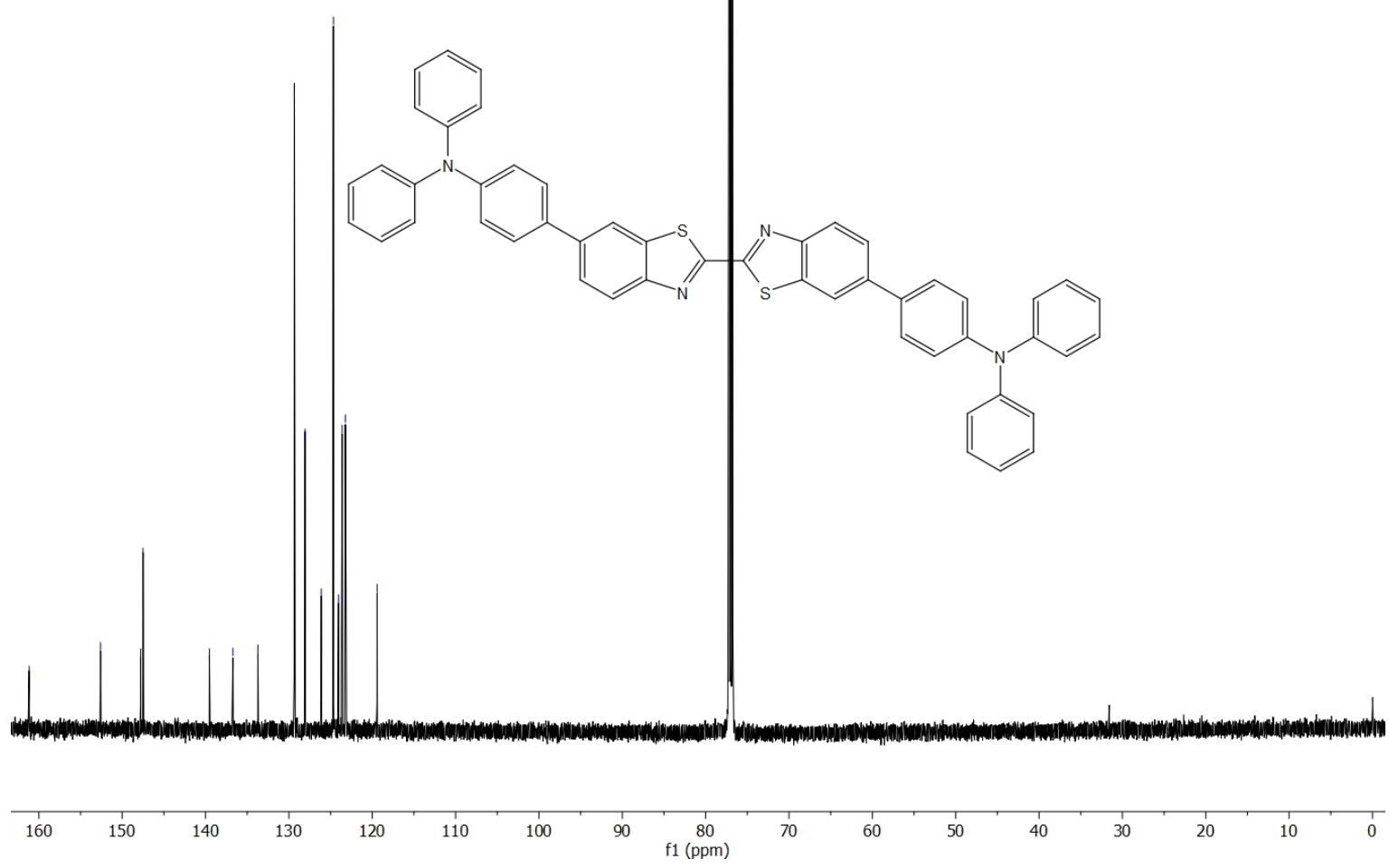

S47 | Supporting Information 
${ }^{1} \mathrm{H}$ NMR spectrum of bisbtz-III (600 $\left.\mathrm{MHz}, \mathrm{CDCl}_{3}\right)$

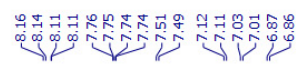

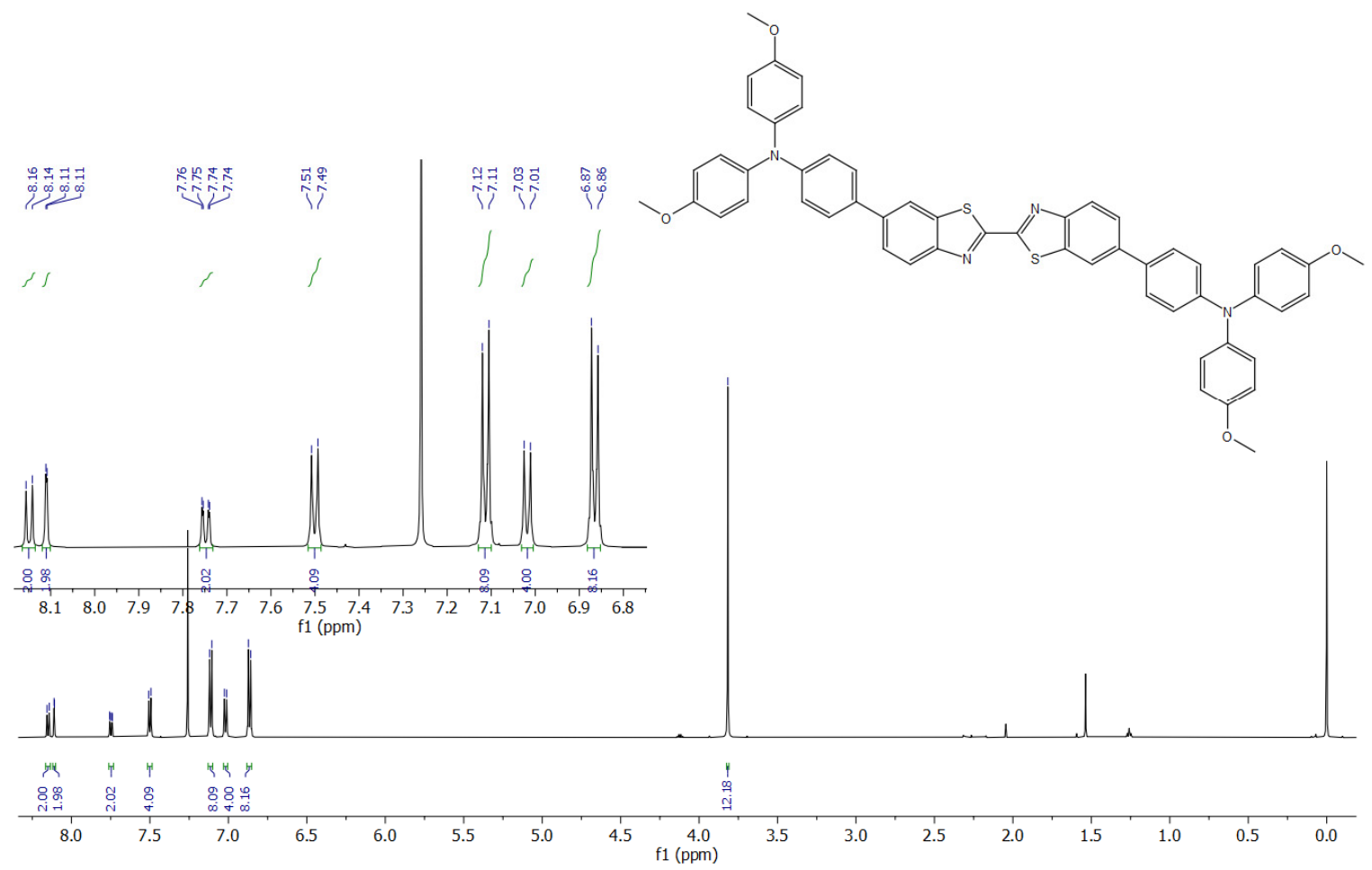

${ }^{13} \mathrm{C}$ NMR spectrum of bisbtz-III (151 $\left.\mathrm{MHz}, \mathrm{CDCl}_{3}\right)$

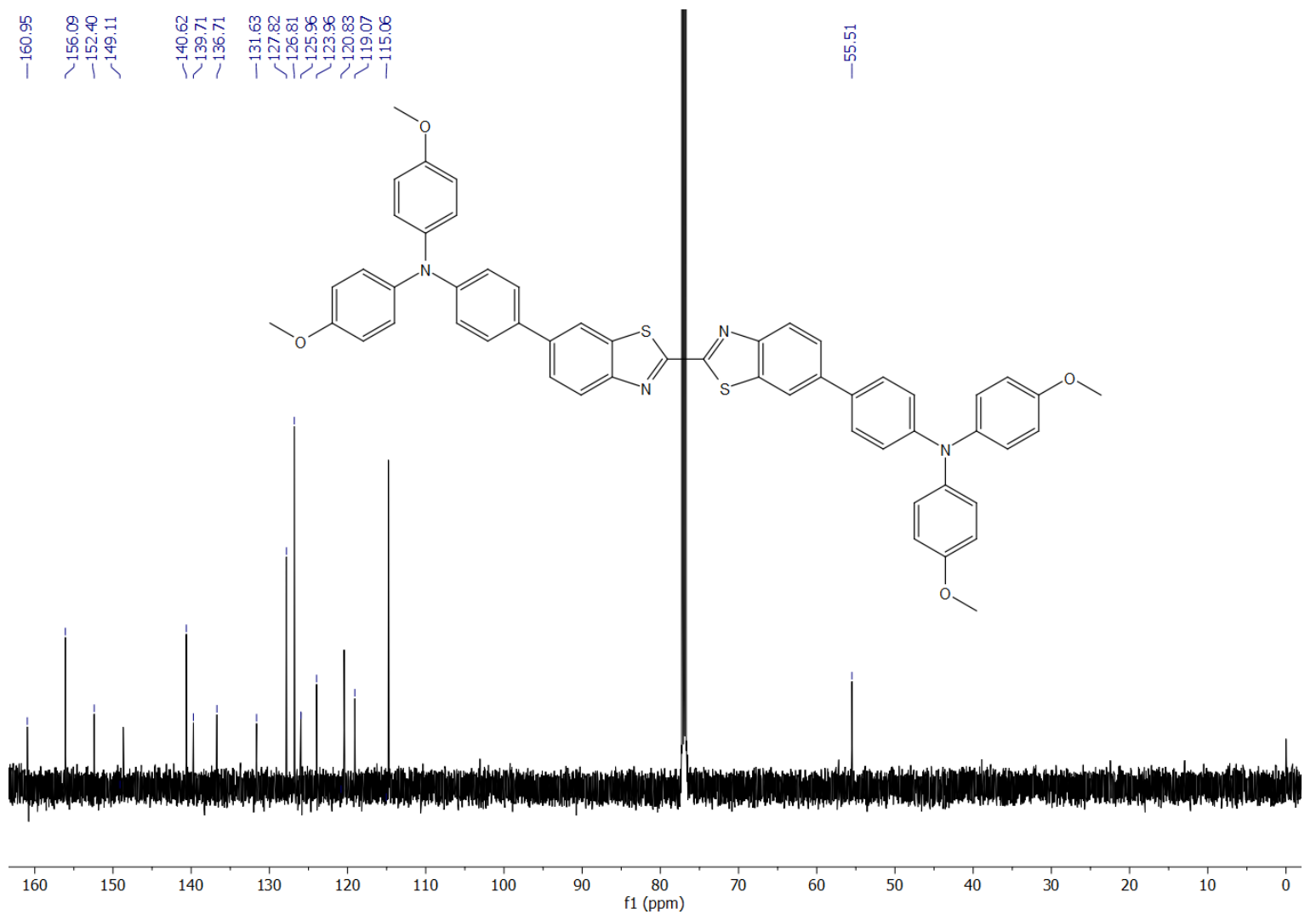

S48 | Supporting Information 
${ }^{1} \mathrm{H}$ NMR spectrum of bisbtz-IV (600 $\left.\mathrm{MHz}, \mathrm{CDCl}_{3}\right)$

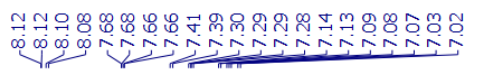
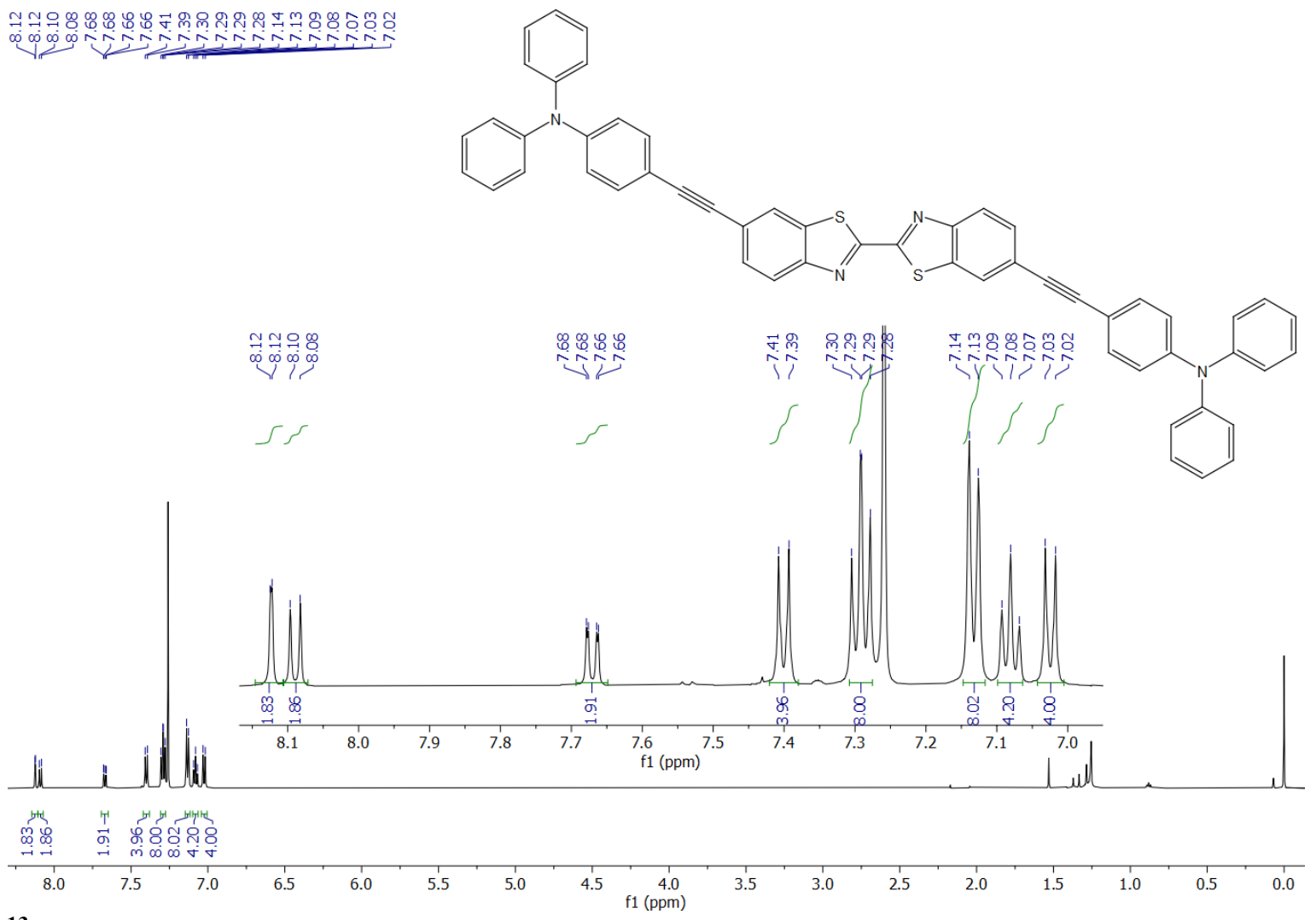

${ }^{13} \mathrm{C}$ NMR spectrum of bisbtz-IV (151 $\left.\mathrm{MHz}, \mathrm{CDCl}_{3}\right)$
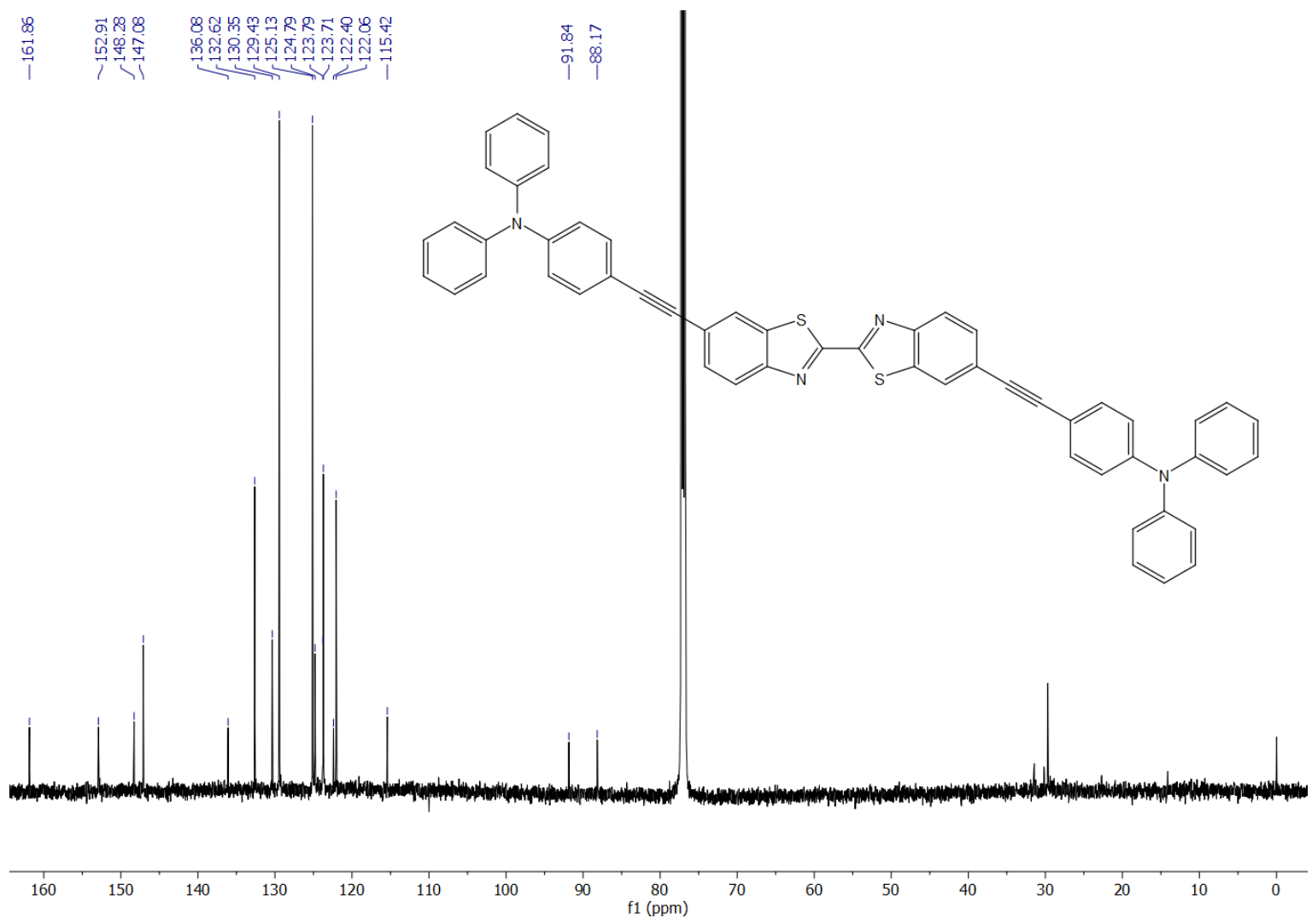

S49 | Supporting Information 
${ }^{1} \mathrm{H}$ NMR spectrum of bisbtz-V (600 $\left.\mathrm{MHz}, \mathrm{CDCl}_{3}\right)$

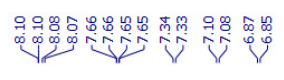

䓴
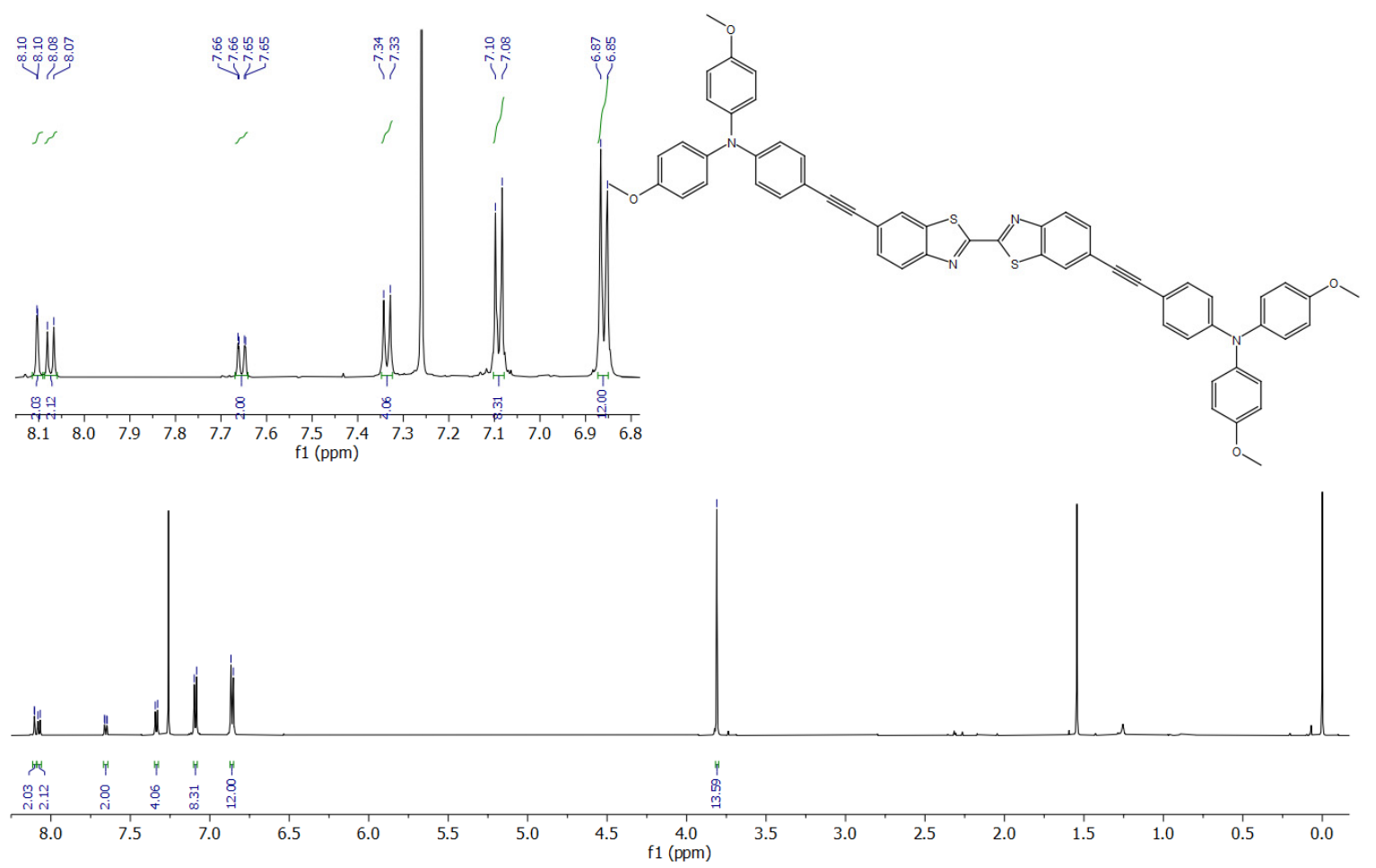

${ }^{13} \mathrm{C}$ NMR spectrum of bisbtz-V (151 $\left.\mathrm{MHz}, \mathrm{CDCl}_{3}\right)$

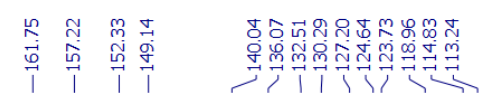

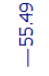

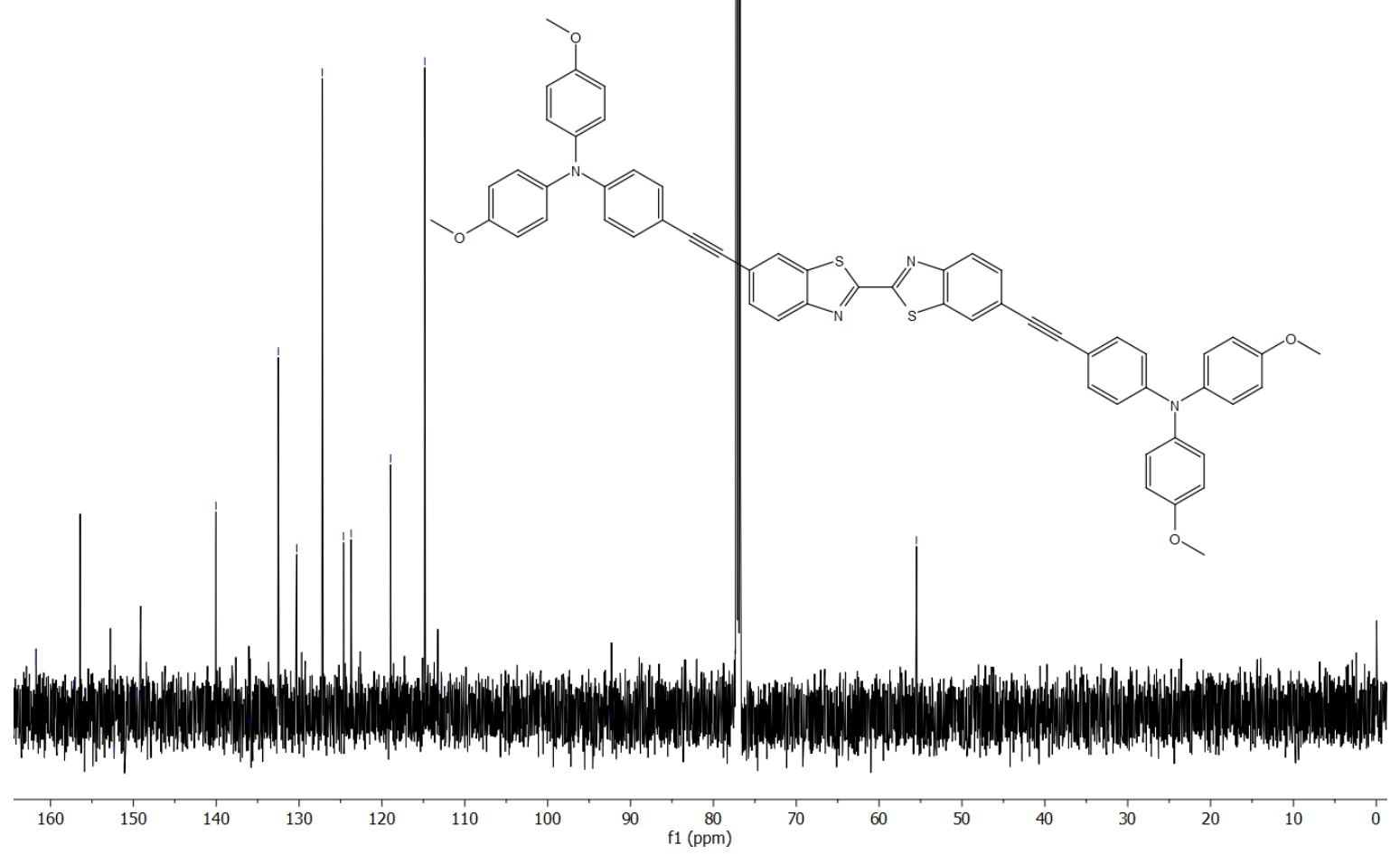

S50 | Supporting Information 
${ }^{1} \mathrm{H}$ NMR spectrum of bisbtz-VI (600 $\left.\mathrm{MHz}, \mathrm{CDCl}_{3}\right)$

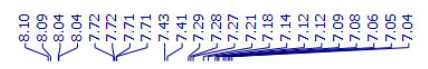
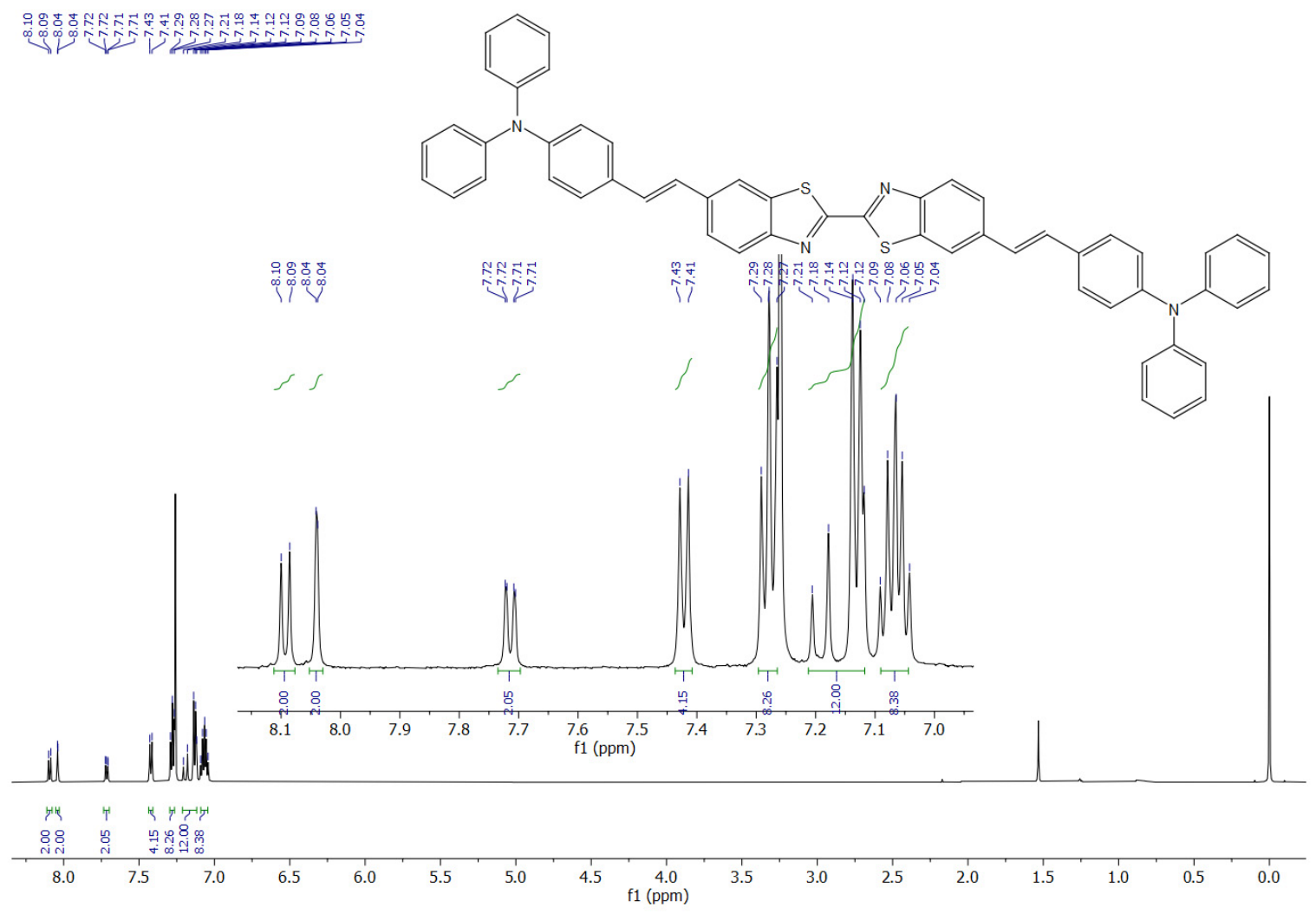

${ }^{13} \mathrm{C}$ NMR spectrum of bisbtz-VI (151 $\left.\mathrm{MHz}, \mathrm{CDCl}_{3}\right)$

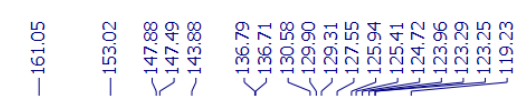

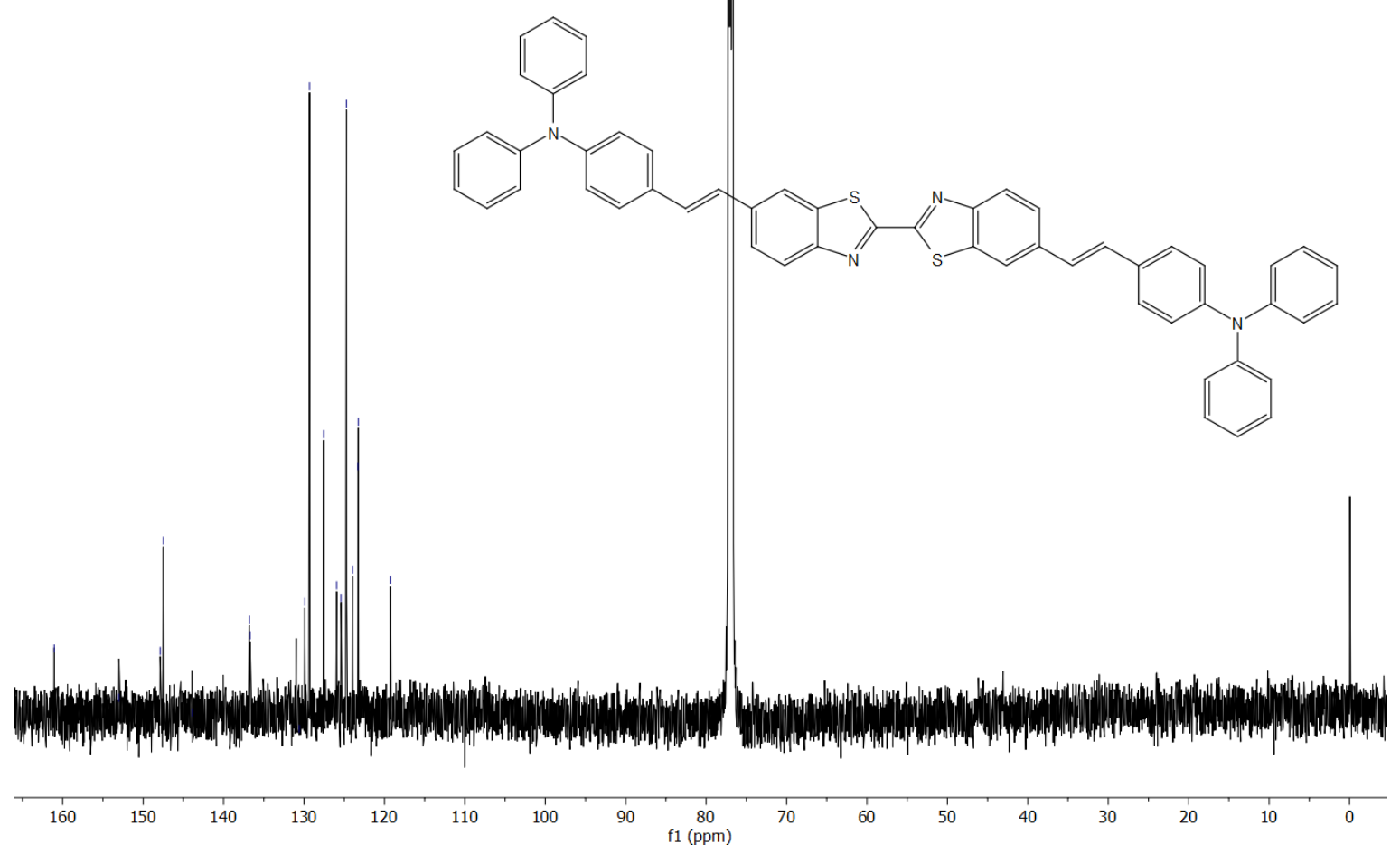

S51 | Supporting Information 
${ }^{1} \mathrm{H}$ NMR spectrum of $\operatorname{ReCl}(\mathrm{CO})_{3}\left(\right.$ bisbtz-I) $\left(600 \mathrm{MHz}, \mathrm{CDCl}_{3}\right)$

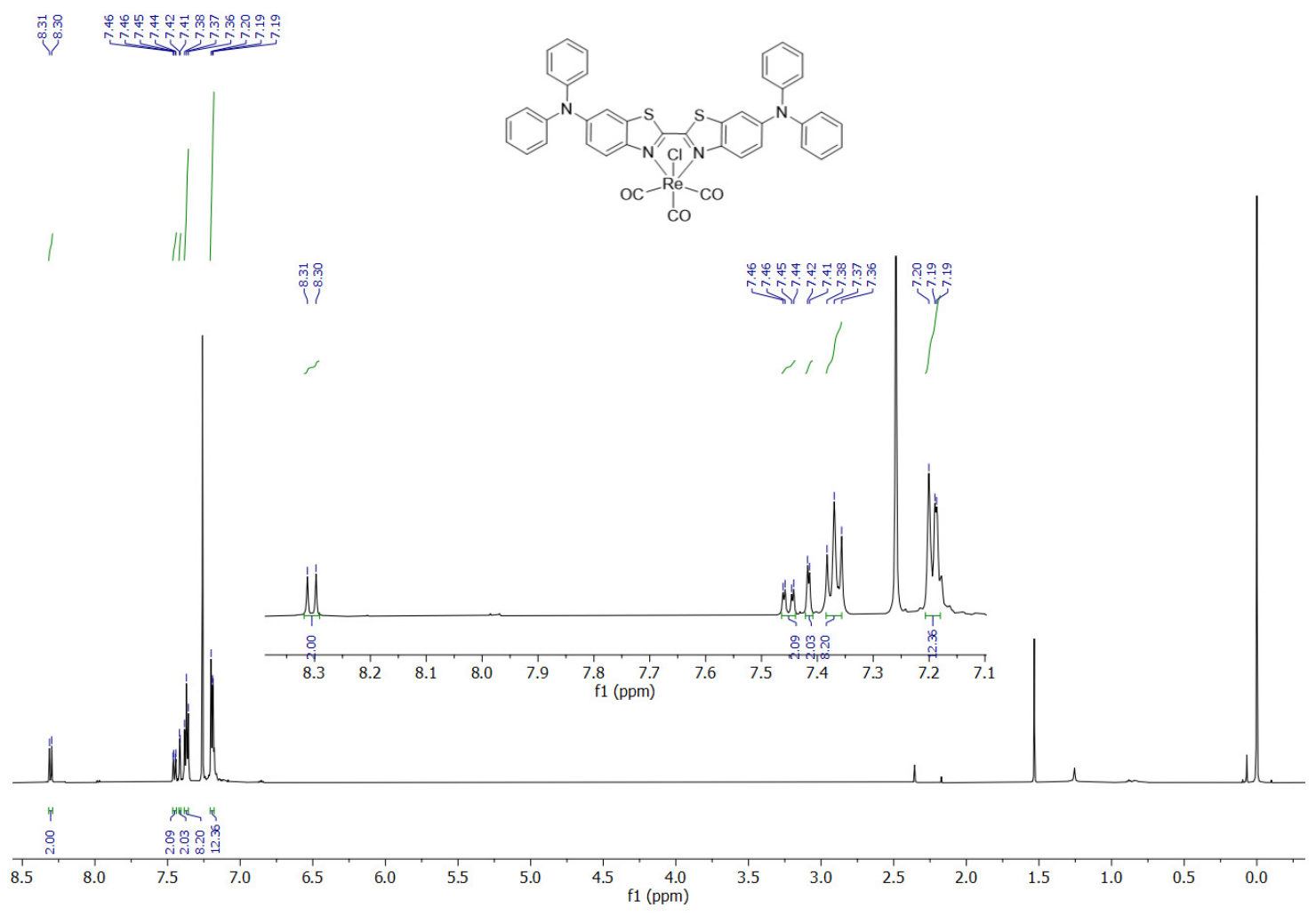

${ }^{13} \mathrm{C}$ NMR spectrum of $\operatorname{ReCl}(\mathrm{CO})_{3}\left(\right.$ bisbtz-I) $\left(151 \mathrm{MHz}, \mathrm{CDCl}_{3}\right)$
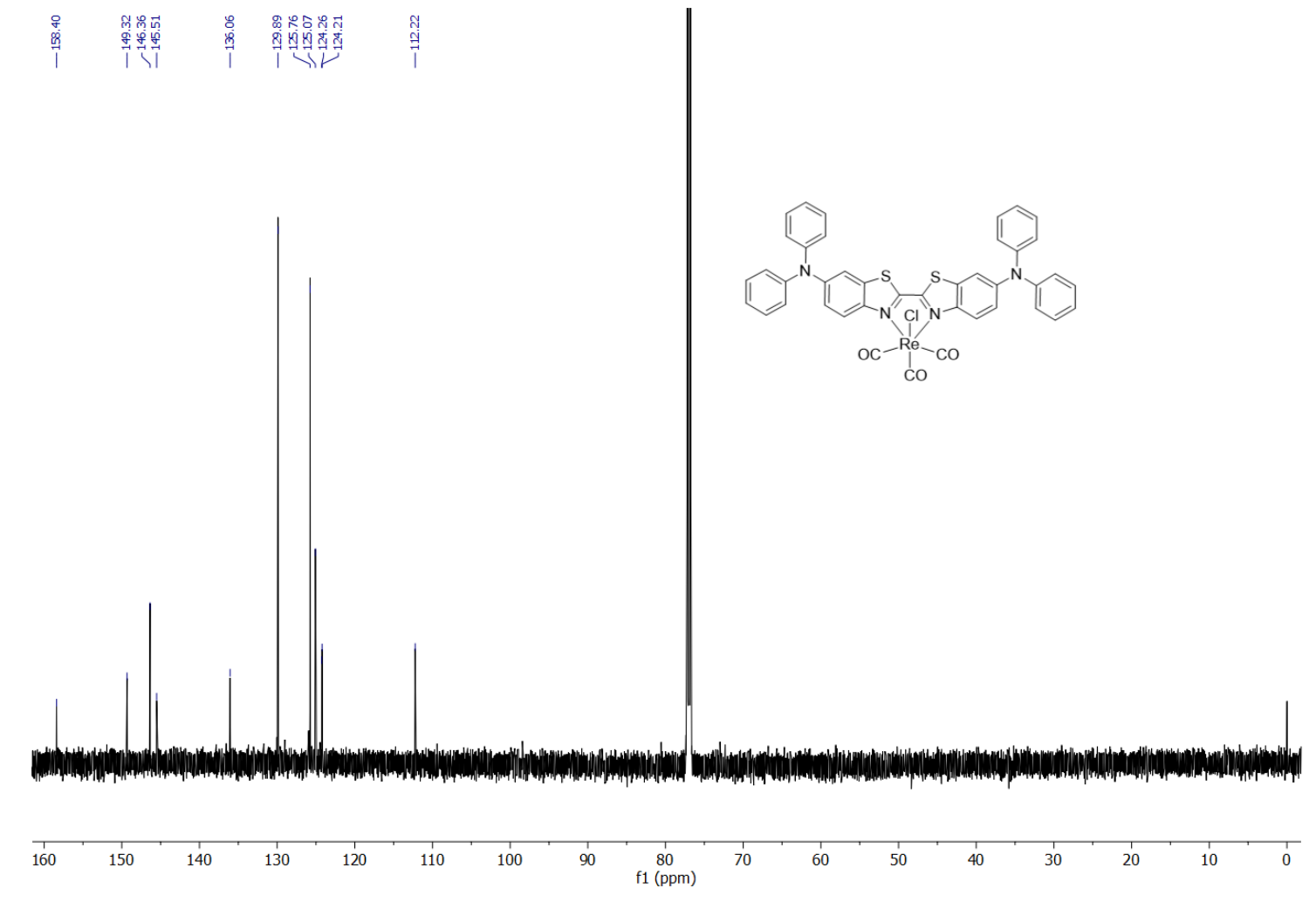
${ }^{15} \mathrm{~N}$ NMR spectrum of $\operatorname{ReCl}(\mathrm{CO})_{3}\left(\right.$ bisbtz-I) $\left(61 \mathrm{MHz}, \mathrm{CDCl}_{3}\right)$

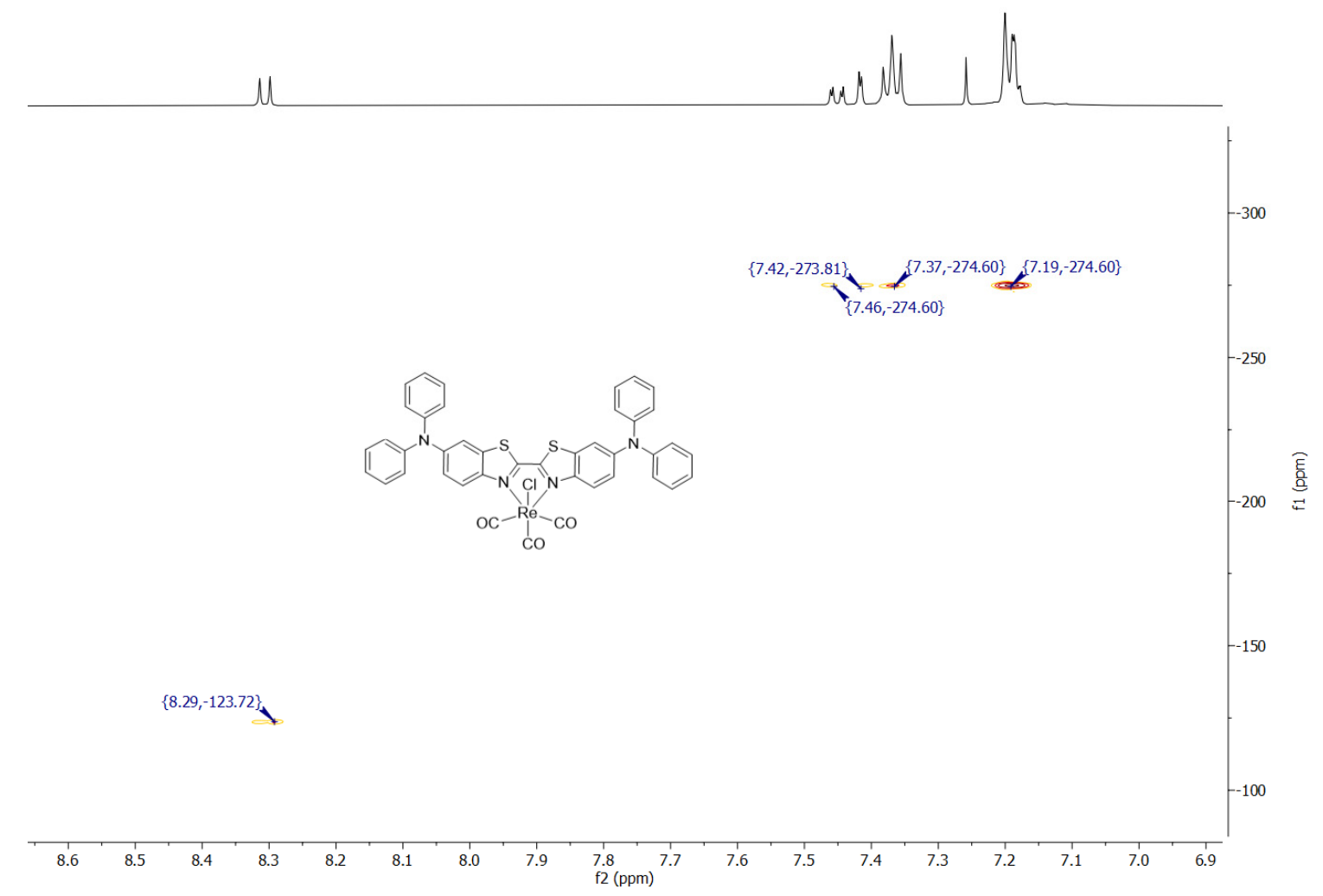

S53 | Supporting Information 


\section{Synthesis of $(E, E)-4,4$ '-[4-( $N, N$-diphenylamino)phenylethenyl]-2,2'-bipyridine}

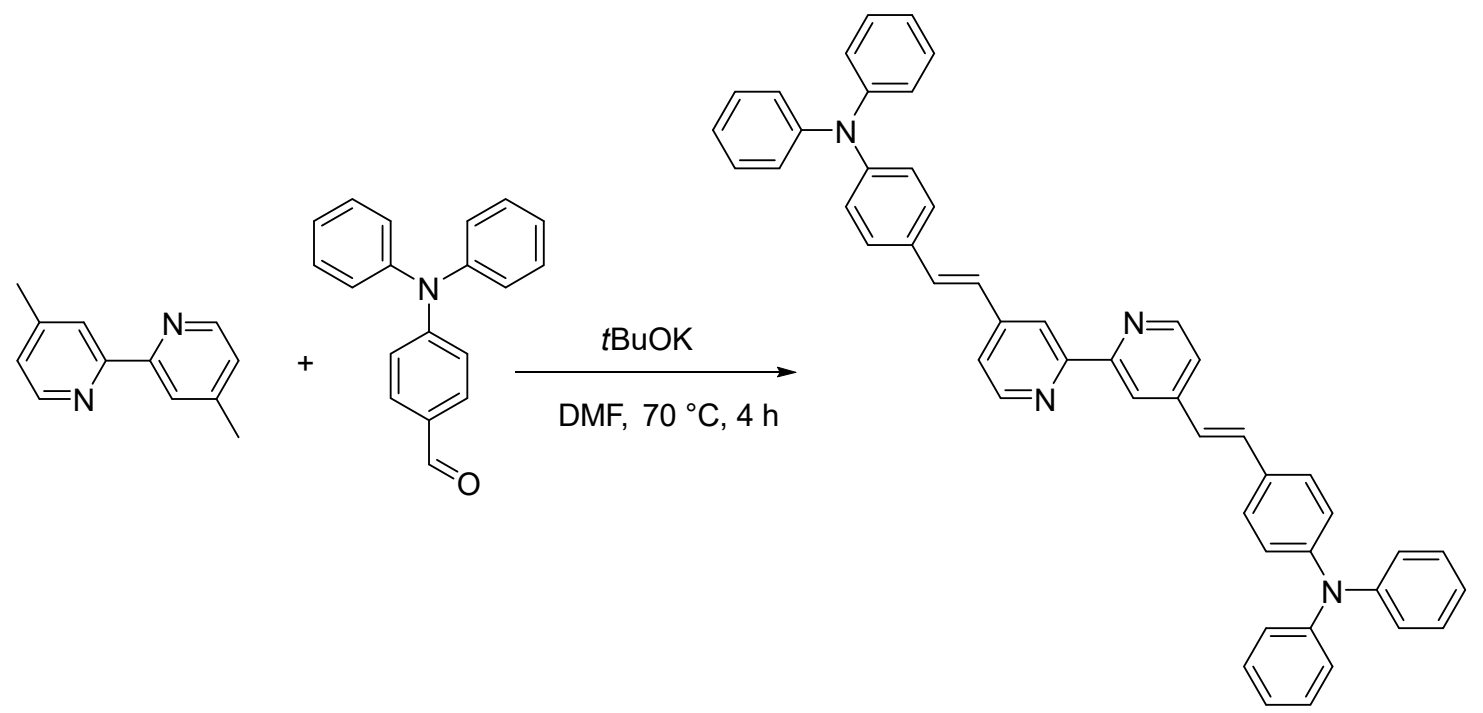

To a flask containing 4,4'-dimethyl-2,2'-bipyridine $(0.50 \mathrm{~g}, 2.7 \mathrm{mmol})$ and potassium tertbutoxide (1.83 g, $16.3 \mathrm{mmol})$ under argon atmosphere, dry DMF $(15 \mathrm{~mL})$ was added through septum via syringe and the resulting mixture was heated to $70{ }^{\circ} \mathrm{C}$ in an oil bath for $1 \mathrm{~h}$. Subsequently, the aldehyde $(1.67 \mathrm{~g}, 6.1 \mathrm{mmol})$ was added as a solid and the resulting mixture was stirred at $70{ }^{\circ} \mathrm{C}$ for 4 hours. After cooling to RT, the mixture was poured into water and the solid collected by filtration. After drying, the product was purified by trituration with boiling methanol. The insoluble part was collected to yield the product as a yellow solid (1.07 g, $56 \%$ ). ${ }^{1} \mathbf{H}$ NMR $\left(600 \mathrm{MHz}, \mathrm{CDCl}_{3}\right) \delta 8.64(\mathrm{~d}, J=5.1 \mathrm{~Hz}, 2 \mathrm{H}), 8.51(\mathrm{~d}, J=1.7 \mathrm{~Hz}, 2 \mathrm{H}), 7.43(\mathrm{~d}, J=8.1 \mathrm{~Hz}, 4 \mathrm{H})$, $7.37(\mathrm{dd}, J=5.2,1.8 \mathrm{~Hz}, 2 \mathrm{H}), 7.29(\mathrm{~d}, J=7.4 \mathrm{~Hz}, 8 \mathrm{H}), 7.13(\mathrm{~d}, J=7.4 \mathrm{~Hz}, 8 \mathrm{H}), 7.08-7.05$ (m, $8 \mathrm{H}), 7.01(\mathrm{~d}, J=16.1 \mathrm{~Hz}, 4 \mathrm{H}) .{ }^{1} \mathrm{H}$ NMR shifts match those reported for this compound in refs. [41] and [42]. 
${ }^{1} \mathrm{H}$ NMR spectrum of $(E, E)-4,4$ '-[4-(N,N-diphenylamino)phenylethenyl]-2,2'-bipyridine (bpy) (600 MHz, $\left.\mathrm{CDCl}_{3}\right)$

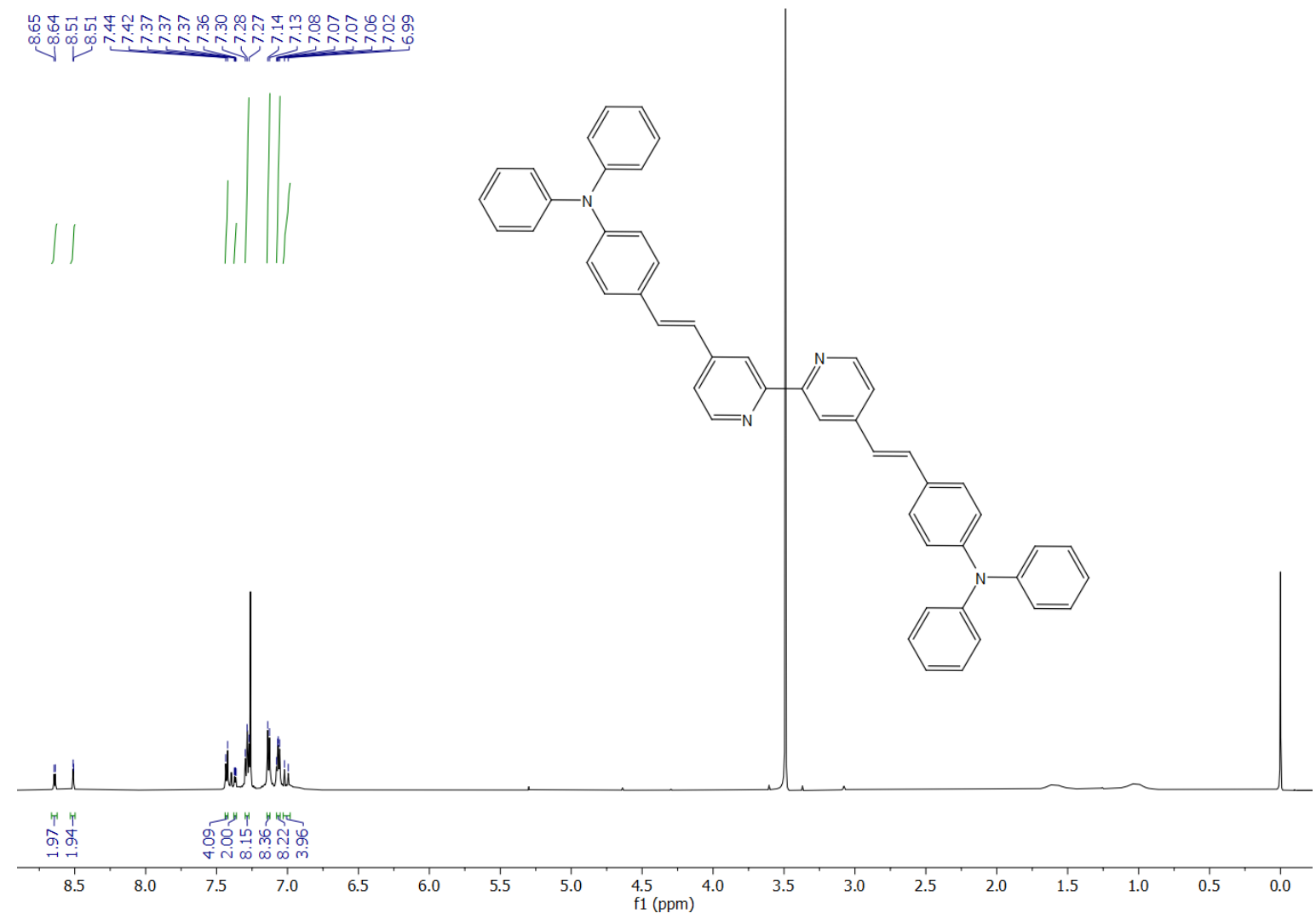




\section{UV-vis absorption and emission spectra}

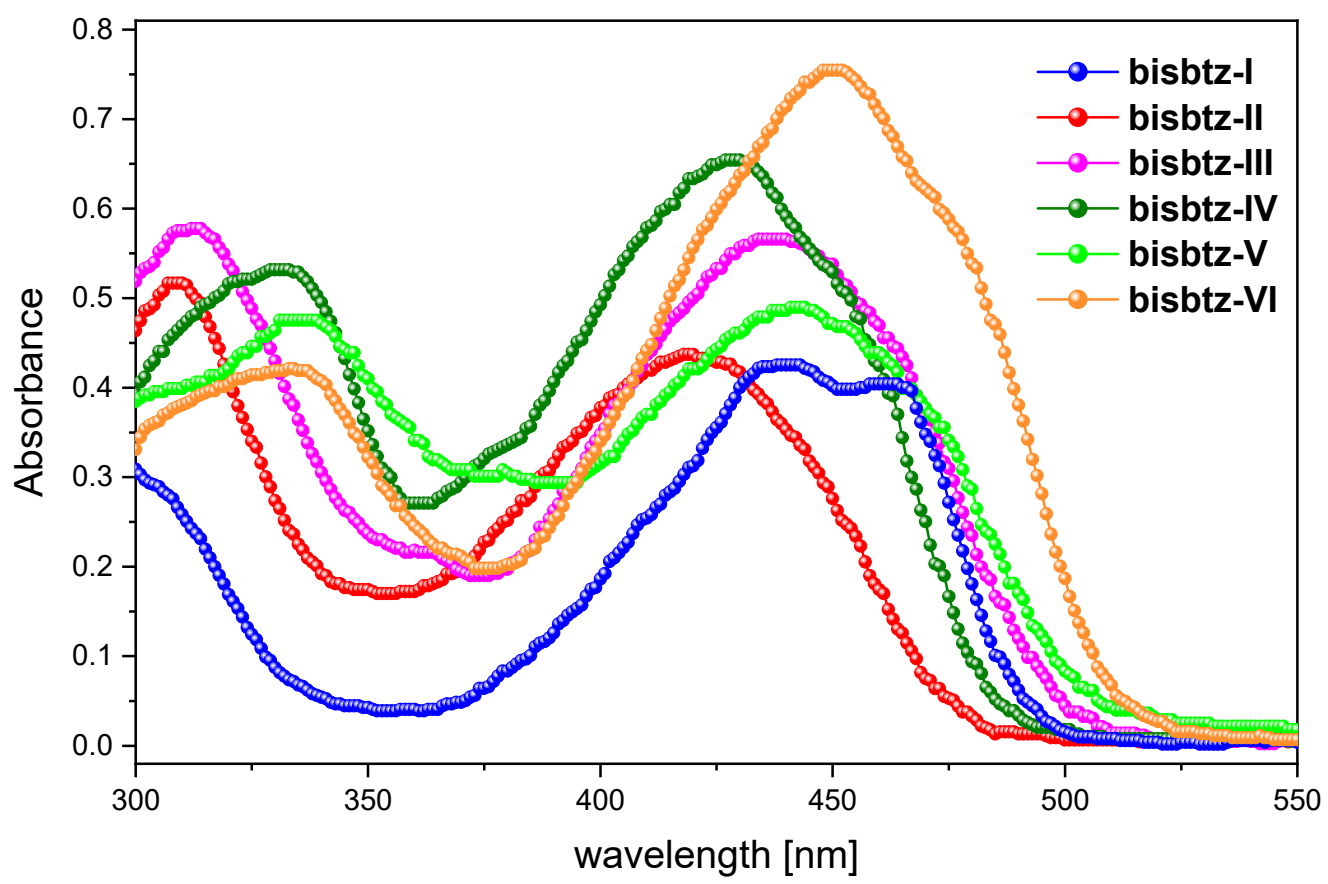

Figure S1. UV-Vis spectra of bisbtz dyes in toluene (measured at $c=1 \times 10^{-5} \mathrm{M}$ )

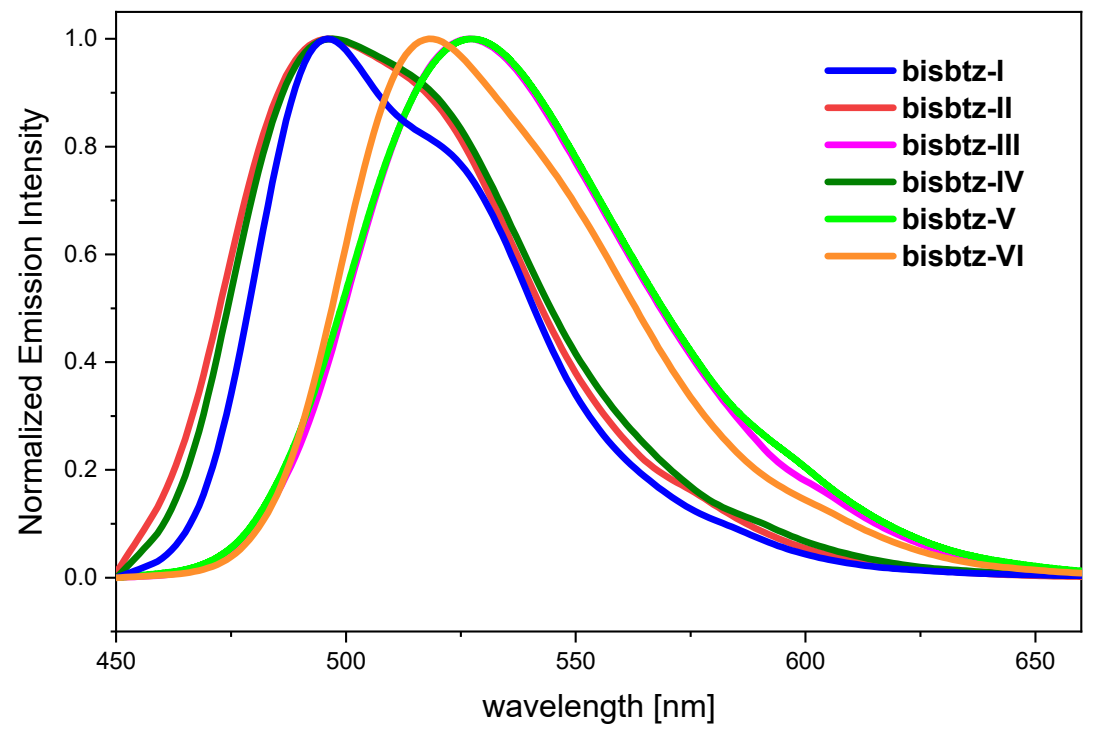

Figure S2. Normalized emission spectra of bisbtz dyes in toluene

(measured at concentrations, at which the absorbance at the excitation wavelength $<0.1$ ) 


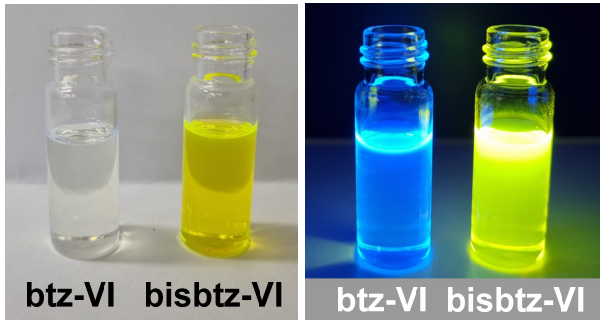

toluene solutions $\left(c=1.10^{-5} \mathrm{M}\right)$
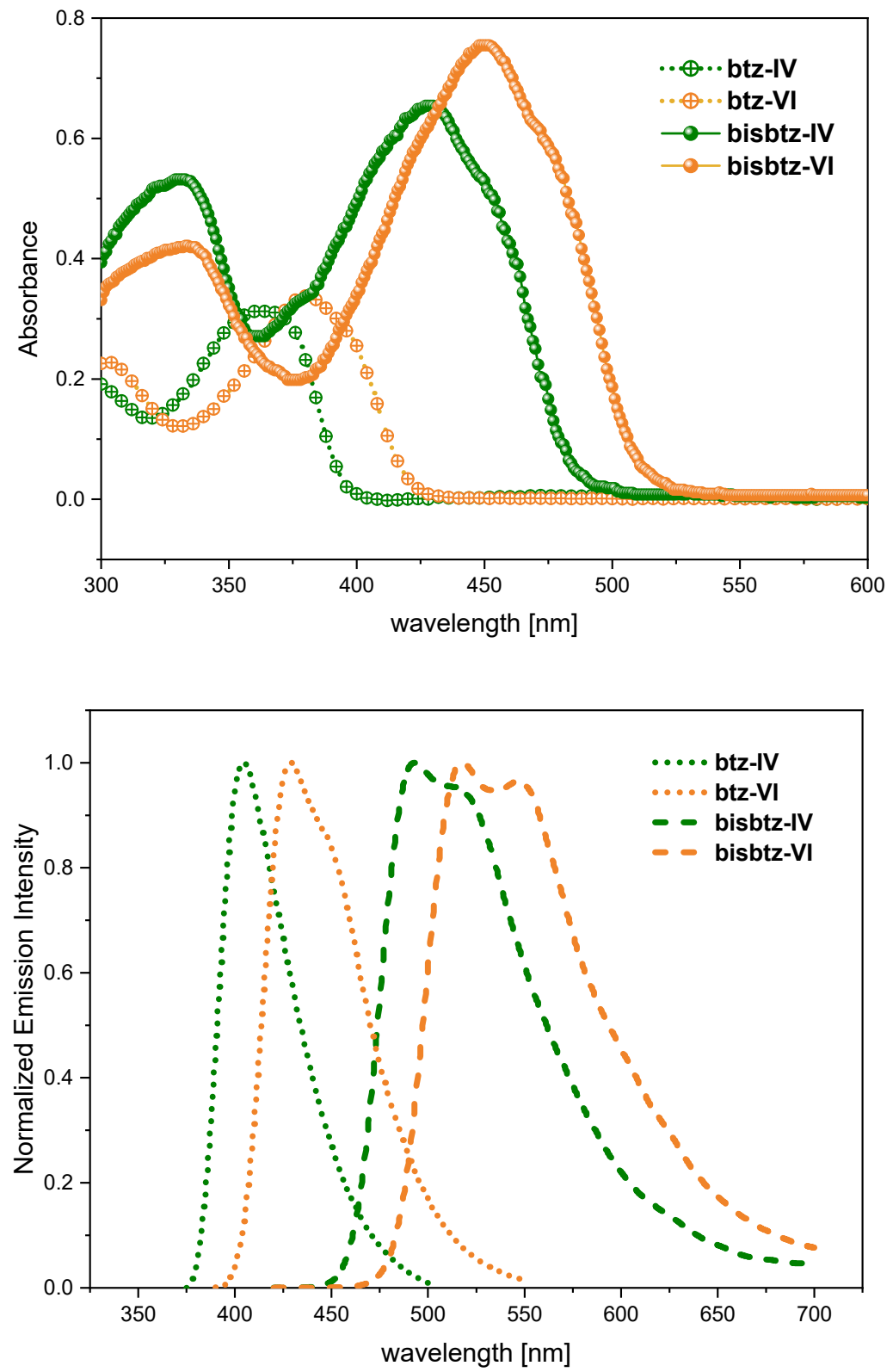

Figure S3. Comparison of absorption and emission spectra of push-pull 2-H-benzothiazoles

(btz-IV, btz-VI) and quadrupolar systems thereof (bisbtz-IV, bisbtz-VI), showing remarkable red-shifts of absorption and emission peaks and increase of extinction coefficients upon homocoupling of two heteroarenes $\left(1 \times 10^{-5} \mathrm{M}\right.$ solutions in toluene $)$. 


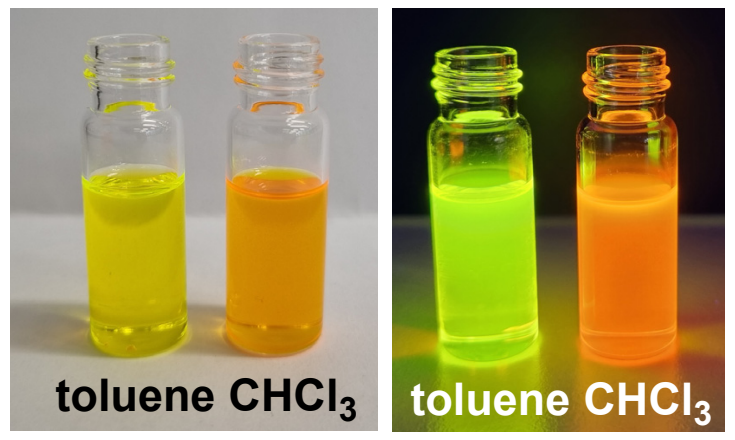

bisbtz-VI solutions $\left(c=1.10^{-5} \mathrm{M}\right)$

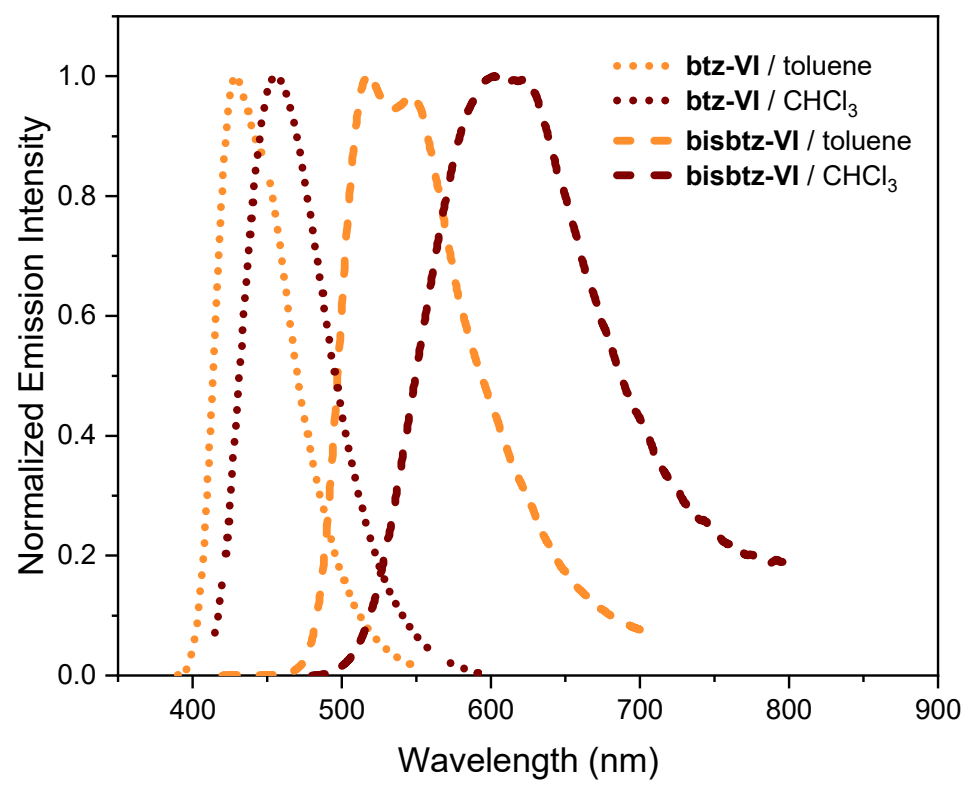

Figure S4. Emission spectra of btz-VI and bisbtz-VI in toluene and $\mathrm{CHCl}_{3}$, demonstrating a pronounced red-shift of emission in bisbtz-VI dye upon increasing solvent polarity. 


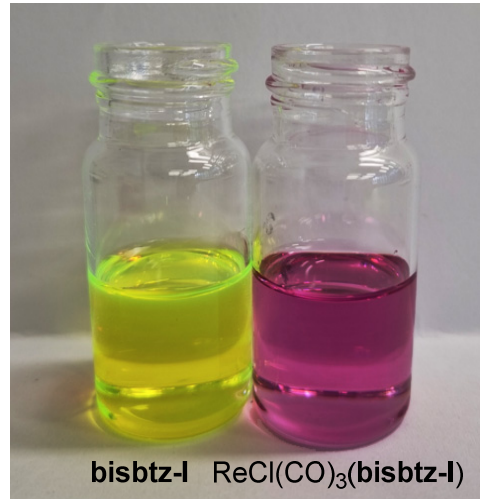

toluene solutions $\left(c=1.10^{-5} \mathrm{M}\right)$

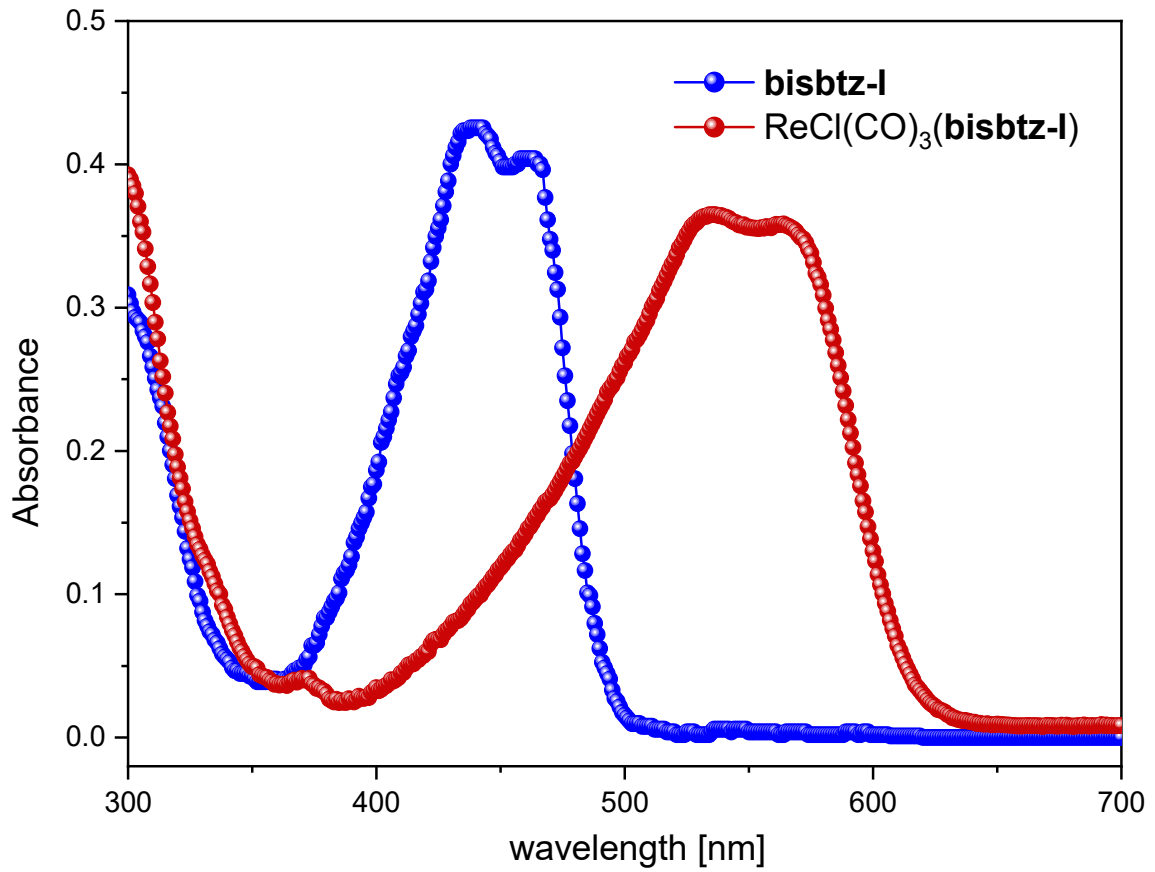

Figure S5. Comparison of UV-vis absorption spectra of bisbtz-I and $\operatorname{ReCl}(\mathrm{CO})_{3}$ (bisbtz-I) (toluene solutions, $c=1 \times 10^{-5} \mathrm{M}$ ) 


\section{Fluorescence quantum yields measurements and TCSPC spectroscopy}

The fluorescence quantum yields were determined by means of the following model: ${ }^{[15]}$

$$
\Phi_{x}=\Phi_{\text {ref }}\left(\frac{\operatorname{grad}_{X}}{\operatorname{grad}_{\text {ref }}}\right)\left(\frac{\eta_{X}^{2}}{\eta_{\text {ref }}^{2}}\right)
$$

where $\Phi$ is the fluorescence quantum yield, grad denotes the gradient from the plot of integrated fluorescence intensity (area under the emission curve) $v s$. absorbance, and $\eta$ is the refractive index of the solvents used in measurement. The subscripts $r e f$ and $X$ stand for the reference and tested dye, respectively.

A series of btz and bisbtz dye solutions in UV-VIS grade toluene $(\eta=1.496)$ or chloroform $(\eta=$ 1.445) was prepared so that the absorbance at excitation wavelength $\left(\lambda_{\mathrm{exc}}=370 \mathrm{~nm}\right.$ for $\mathbf{b t z}$ and $\lambda_{\mathrm{exc}}=410 \mathrm{~nm}$ for bisbtz) did not exceed 0.1 . The standard - perylene $\left(\mathrm{QY}, \lambda_{\mathrm{exc}}=410 \mathrm{~nm}\right.$; $\Phi_{\mathrm{ref}}=$ $0.94)^{[16]}$ was dissolved in cyclohexane $(\eta=1.427)$. Emission intensity (measured as area under emission curve) was plotted against absorbance at $\lambda_{\text {exc }}$ and the slope obtained was used for quantum yield calculation. The quantum yields are summarized in Table 1 in the main text.

The time resolved fluorescence measurements were performed by means of Time Correlated Single Photon Counting (TCSPC) spectroscopy. ${ }^{[17]}$ The samples were excited at $400 \mathrm{~nm}$ using ps diode lasers and the detection was made under magic angle conditions. The Instrument's Response Function (IRF) of the system was $\sim 80$ ps. 


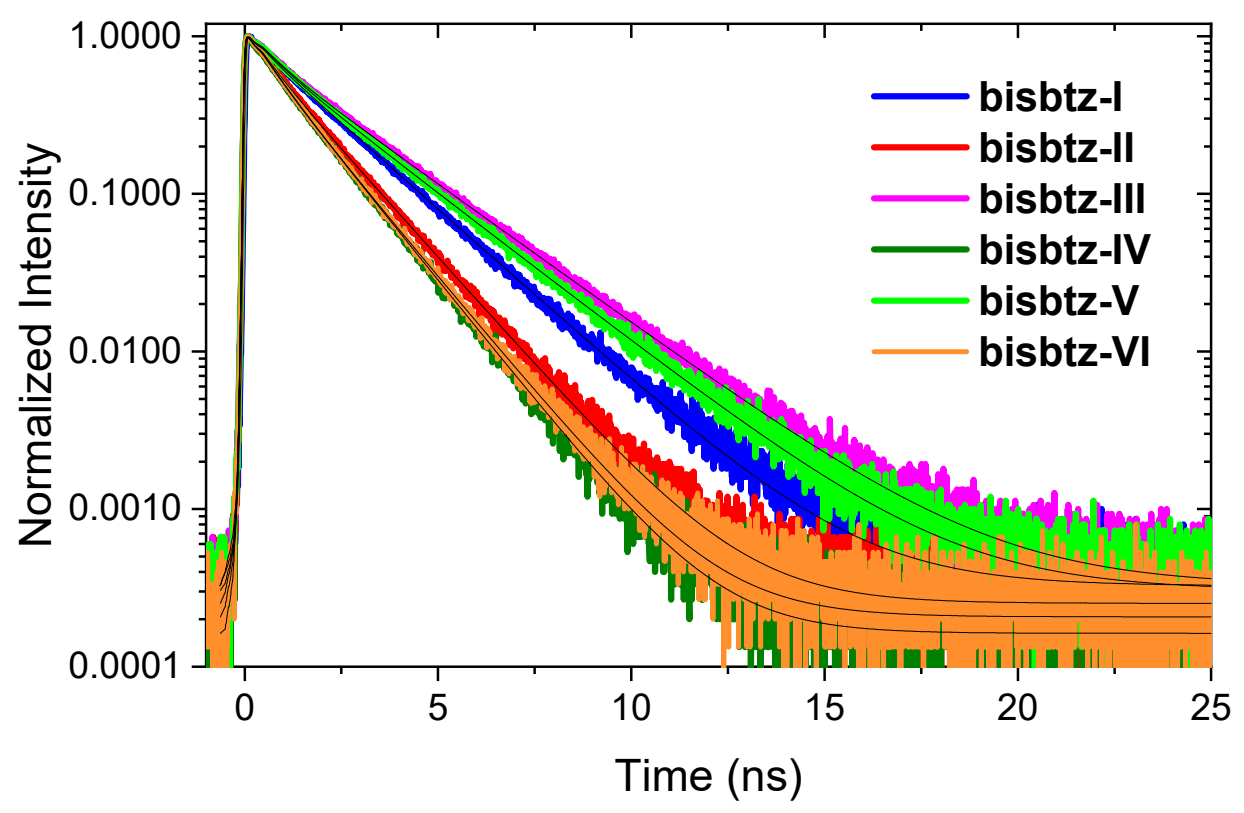

Figure S6. Nanosecond fluorescence dynamics for the bisbtz dyes in toluene solution $\left(c=5 \times 10^{-}\right.$ $\left.{ }^{6} \mathrm{M}\right)$. The excitation wavelength was $400 \mathrm{~nm}$ and the detection was at the wavelength of emission maxima.

Table S1. Fitting parameters for the nanosecond (ns) fluorescence dynamics for the bisbtz dyes in toluene solution $\left(c=5 \times 10^{-6} \mathrm{M}\right)$. The fitting was realized by a bi-exponential function taking into account the instrument's response function.

$\begin{array}{ccccccc}\text { dye } & \boldsymbol{\lambda}_{\text {det }}(\mathbf{n m}) & \mathbf{A}_{\mathbf{1}} & \boldsymbol{\tau}_{\mathbf{1}}(\mathbf{n s}) & \mathbf{A}_{2} & \boldsymbol{\tau}_{\mathbf{2}}(\mathbf{n s}) & <\boldsymbol{\tau}>(\mathbf{n s}) \\ \text { bisbtz-I } & 495 & 0.17 & 0.36 & 0.83 & 1.99 & 1.70 \\ \text { bisbtz-II } & 495 & 0.22 & 0.50 & 0.78 & 1.59 & 1.34 \\ \text { bisbtz-III } & 527 & 0.24 & 0.60 & 0.76 & 2.46 & 2.00 \\ \text { bisbtz-IV } & 496 & 0.21 & 0.47 & 0.79 & 1.42 & 1.22 \\ \text { bisbtz-V } & 527 & 0.23 & 0.65 & 0.77 & 2.30 & 1.92 \\ \text { bisbtz-VI } & 517 & 0.27 & 0.61 & 0.73 & 1.48 & 1.24\end{array}$




\section{TPEF measurements}

The two-photon absorption (TPA) properties of selected dyes were studied via a twophoton excited fluorescence (TPEF) technique using femtosecond (fs) pulsed excitation. ${ }^{[18]-[20]} \mathrm{A}$ mode-locked Ti:Sapphire fs laser, emitting 80 fs pulses tunable from 730 to $870 \mathrm{~nm}$ with $80 \mathrm{MHz}$ repetition frequency, has been used as the excitation source. The laser beam passed through a 5fold expanding telescope in order to achieve uniform illumination at the back of the microscope objective lens (16-fold), used to focus the fs laser beam in the samples. The samples were measured in quartz cuvettes as $2 \times 10^{-5} \mathrm{M}$ solutions of the dyes in toluene. Three linear motorized stages (Newport) driven by a three-axis controller (Newport) have been utilized to move each sample towards the excitation focal point. The TPA-induced fluorescence has been collected backwards and was separated by the laser beam via a dichroic mirror with a high-pass region (cold mirror) and a set of filters. Finally, a photomultiplier connected to photon-counting electronics (Becker-Hickl) was used to detect the emitted fluorescence. Rhodamine $6 \mathrm{G}$ in $\mathrm{MeOH}\left(2 \times 10^{-5} \mathrm{M}\right)$ has been used as the reference sample while the measured scattering intensity from the solvent has been subtracted. The calculations of the TPA cross sections have been made after plotting the TPEF intensity as a function of the excitation intensity in order to confirm the occurrence of the square-law dependence of ITPEF on the excitation power.

The $\delta_{\text {TPA }}$ values of samples $(X)$ were determined through the equation:

$$
\delta_{T P A, X}=\frac{k_{r e f} \delta_{r e f} \Phi_{r e f} n_{r e f} C_{r e f} P_{r e f}^{2}}{k_{X} \Phi_{X} n_{X} C_{X} P_{X}^{2}} \frac{I_{X}}{I_{r e f}}
$$

where $k$ is the fluorescence collection efficiency, $\Phi$ is the two-photon fluorescence quantum yield, $n$ the refractive index, $C$ the concentration of the solutions, $P$ the incident power and $I$ is the intensity of two-photon excited fluorescence. Rhodamine 6G, having a well-known TPA spectrum was used as reference. ${ }^{[20]}$ Finally, for the calculation of $\delta$ тРА, the fluorescence quantum yield upon two-photon excitation was assumed to be the same as that upon one-photon excitation, following the analysis of $\mathrm{Xu}$ et al. ${ }^{[18]}$ 


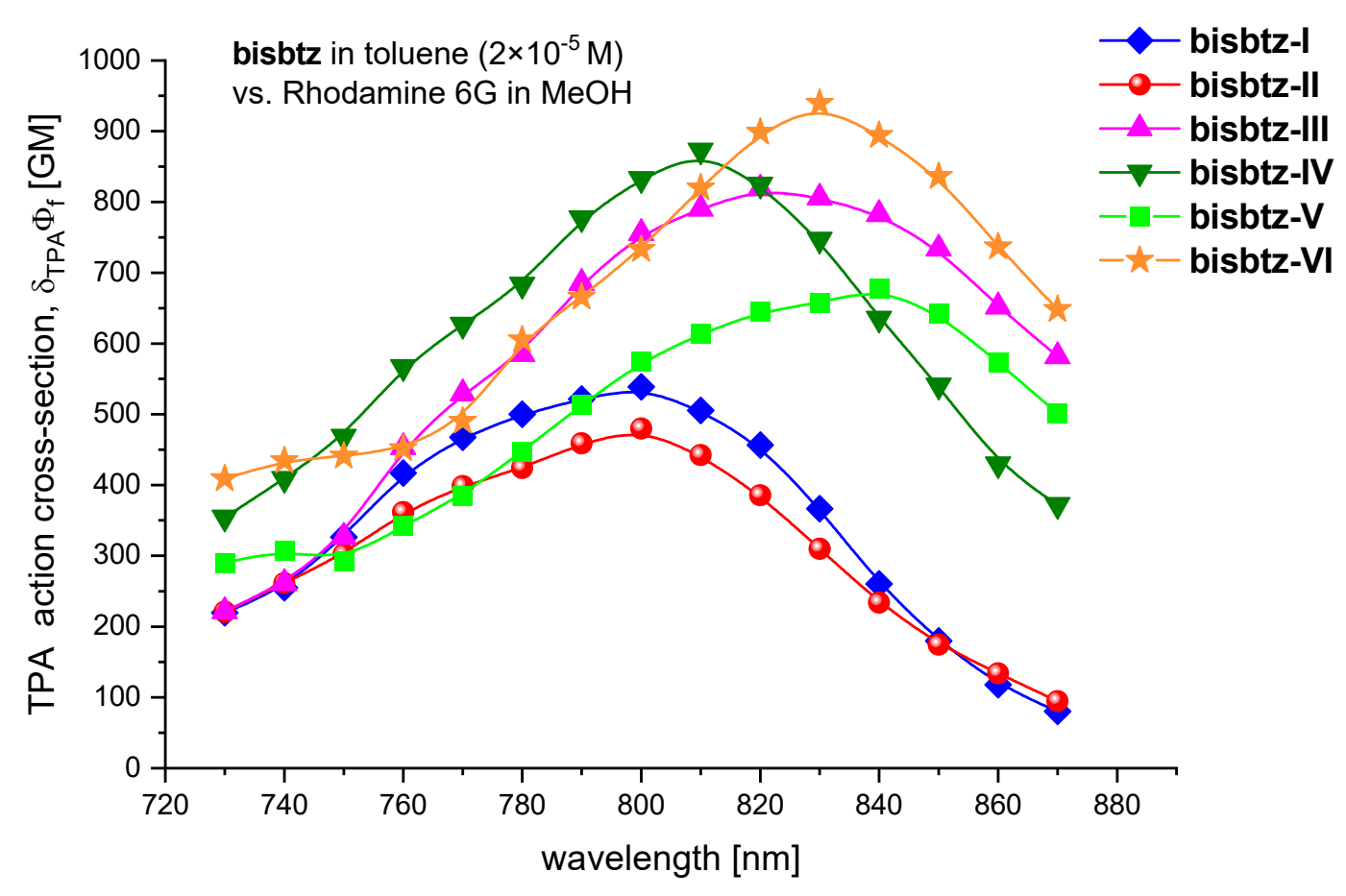

Figure S7. TPA action cross-sections $\left(\delta_{\mathrm{TPA}} \Phi_{\mathrm{f}}\right)$ of bisbtz dyes in toluene

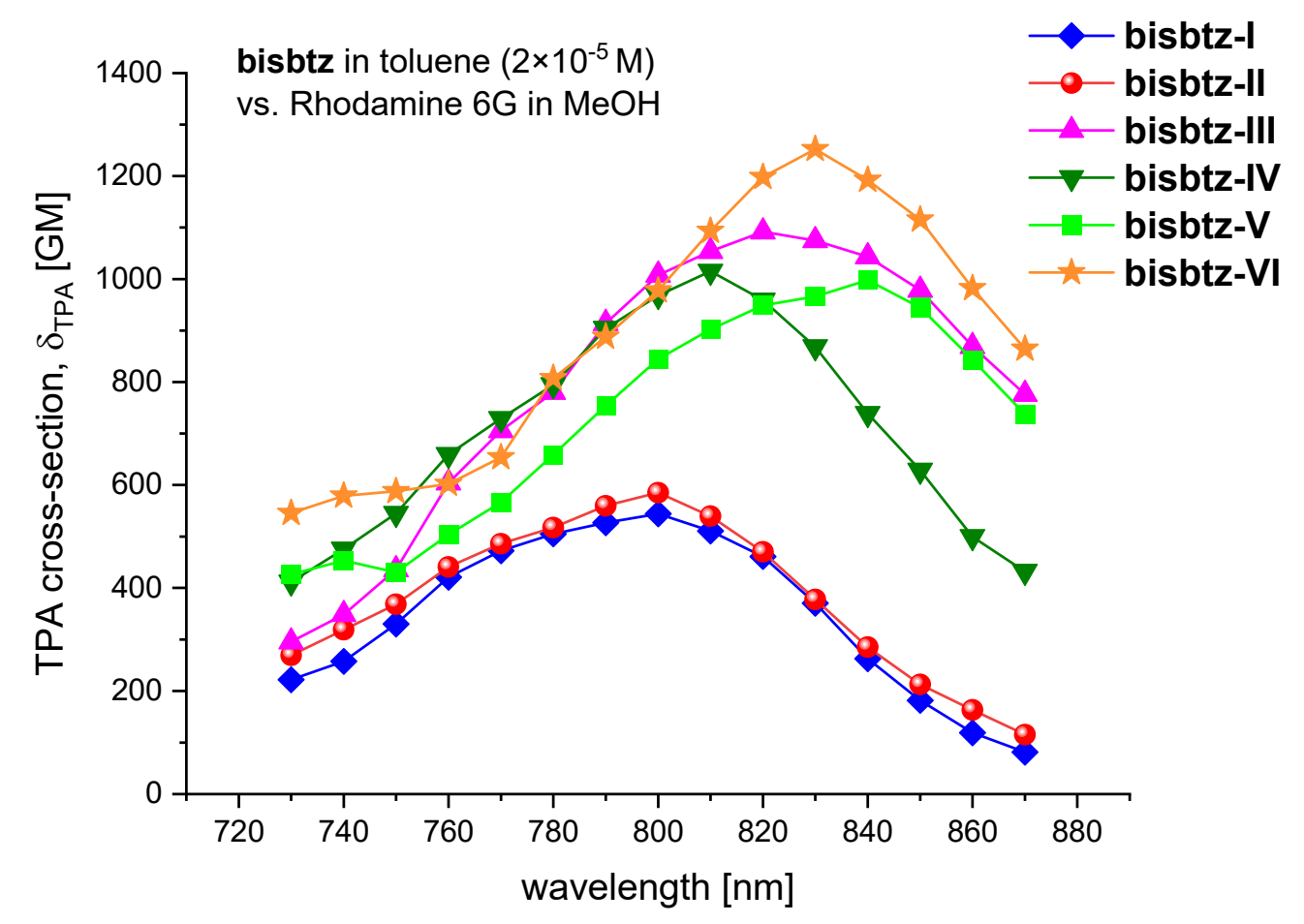

Figure S8. TPA cross-sections ( $\left.\delta_{\mathrm{TPA}}\right)$ of bisbtz dyes in toluene 

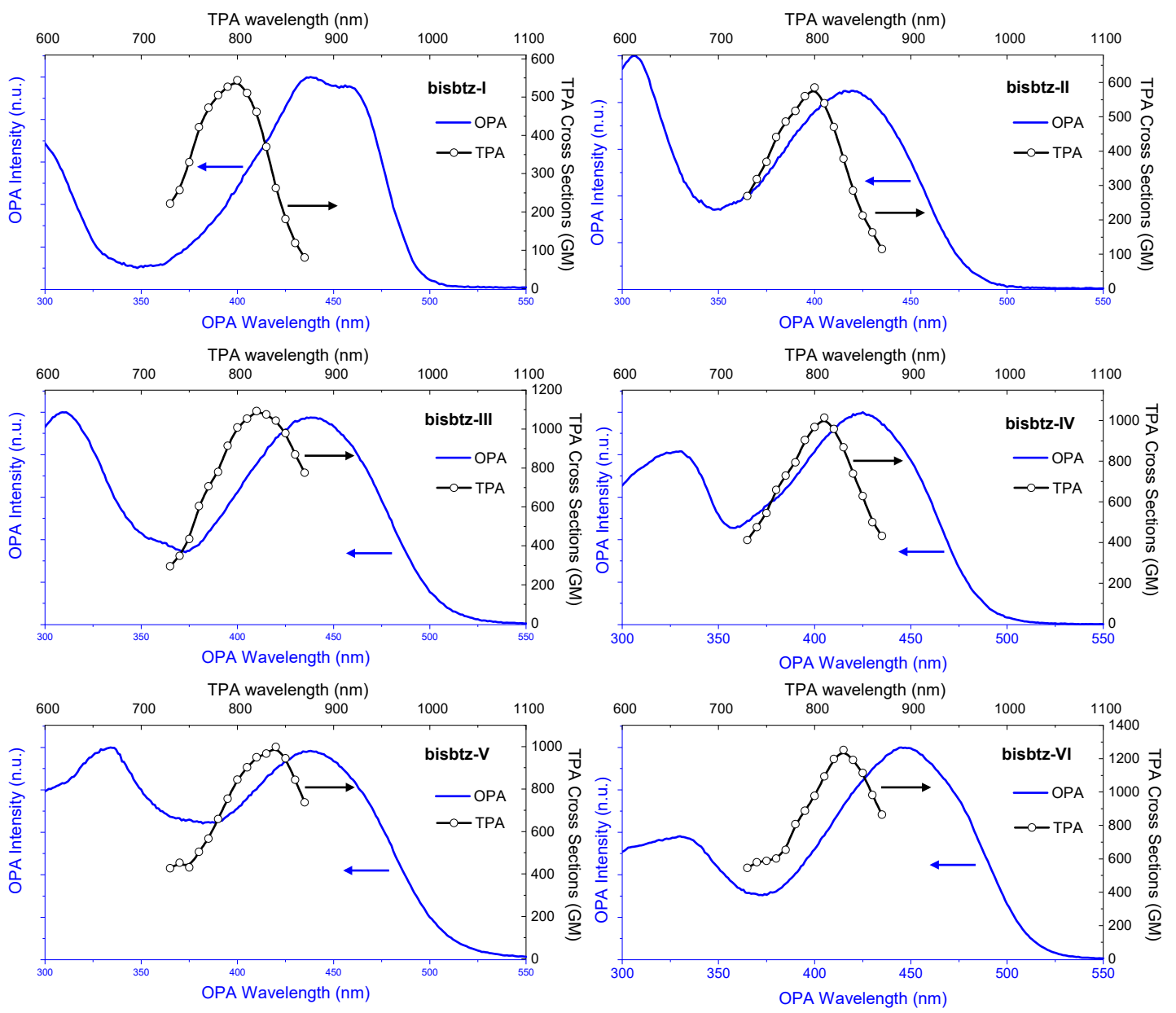

Figure S9. Overlap of one-photon (OPA) and two-photon (TPA) absorption spectra of bisbtz in toluene, confirming the quadrupolar nature of dyes with a deeper $\mathrm{S}_{0} \rightarrow \mathrm{S}_{2}$ transition as compared to OPA $\left(\mathrm{S}_{0} \rightarrow \mathrm{S}_{1}\right)$ process. 

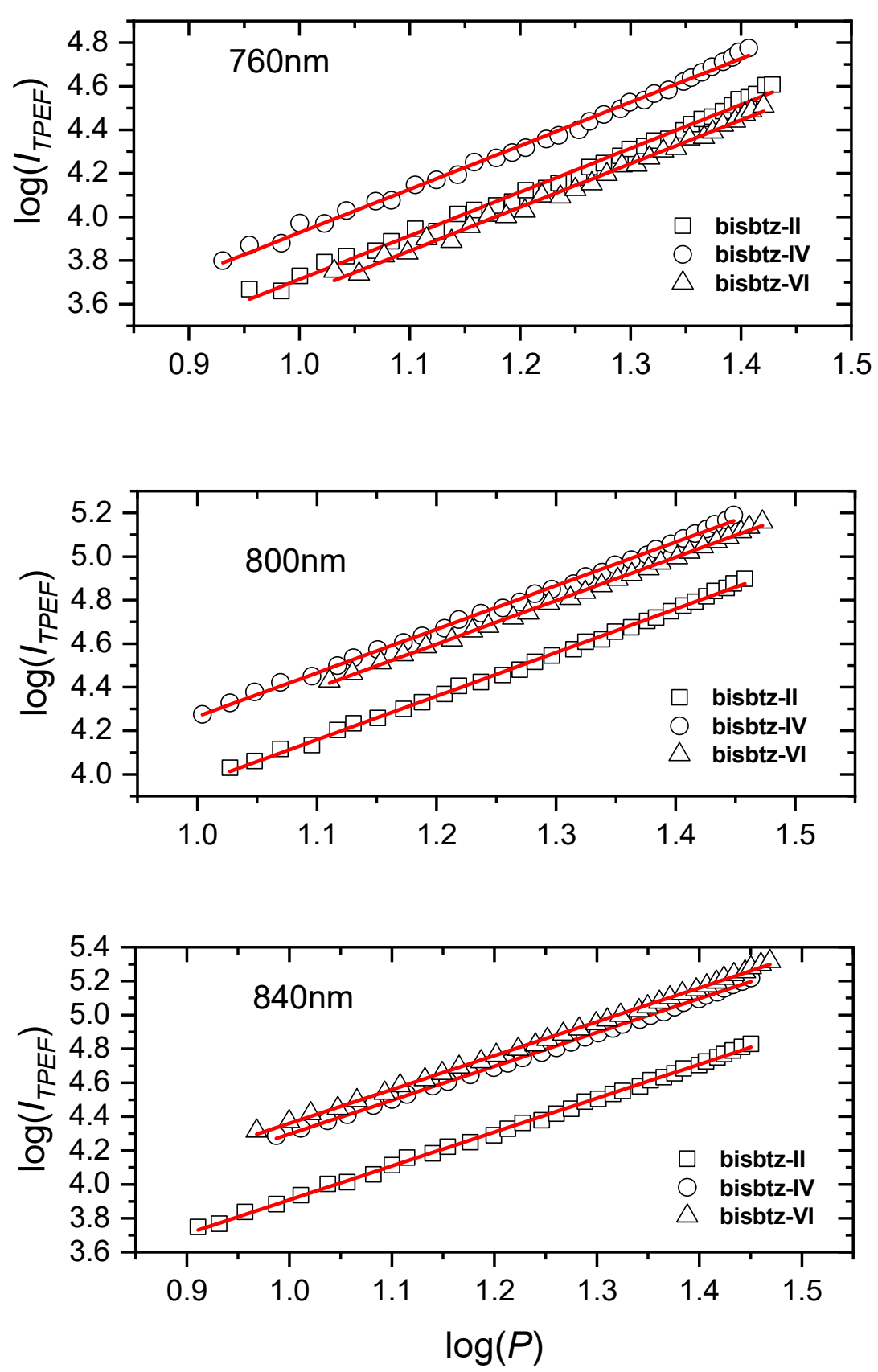

Figure S10. Plot of $\log$ (TPEF intensity) vs. $\log ($ laser power) for selected bisbtz dye solutions. The slopes in all cases were exactly $2.00\left(\mathrm{R}^{2}>0.99\right)$ confirming the quadratic law dependence and assuring that emission is due to a two-photon induced process. 

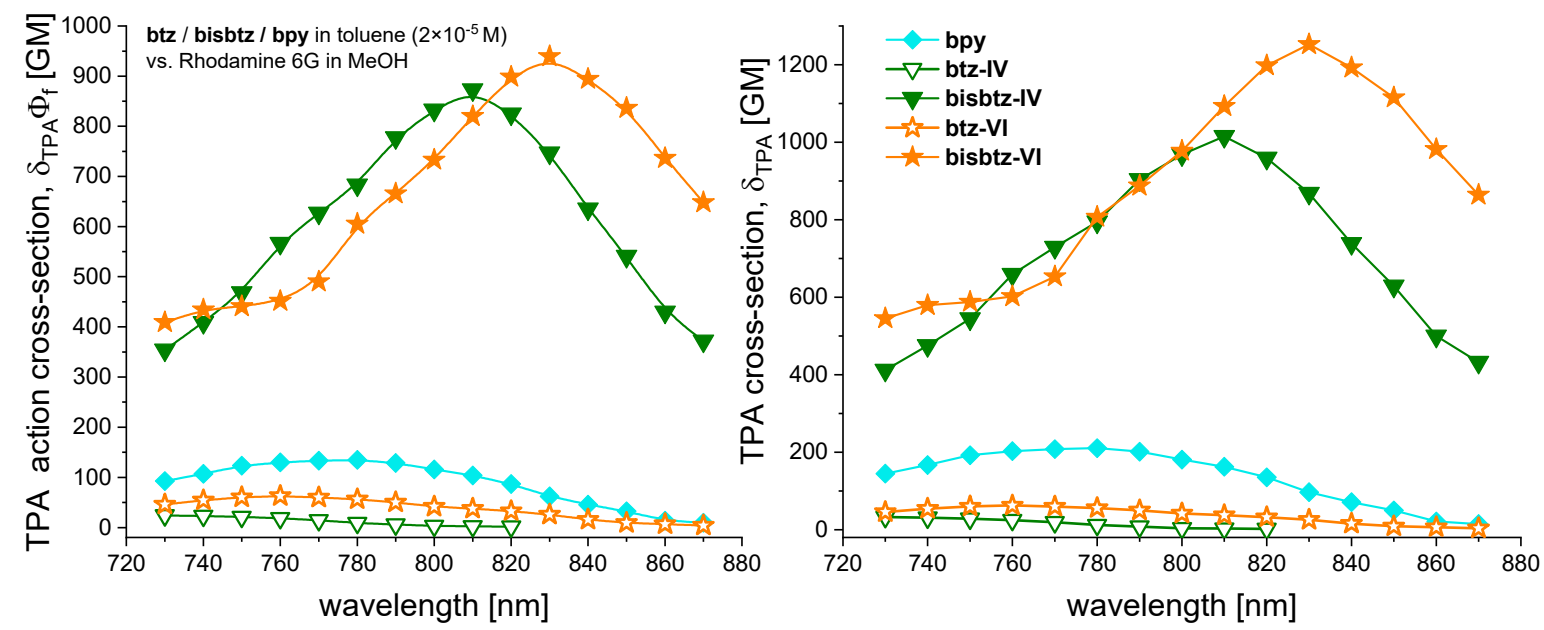

Figure S11. Comparison of TPA cross-sections for bpy, pertinent push-pull 2-H-benzothiazoles (btz-IV, btz-VI) and quadrupolar systems thereof (bisbtz-IV, bisbtz-VI) in toluene, demonstrating remarkable TPA enhancement in the latter series.

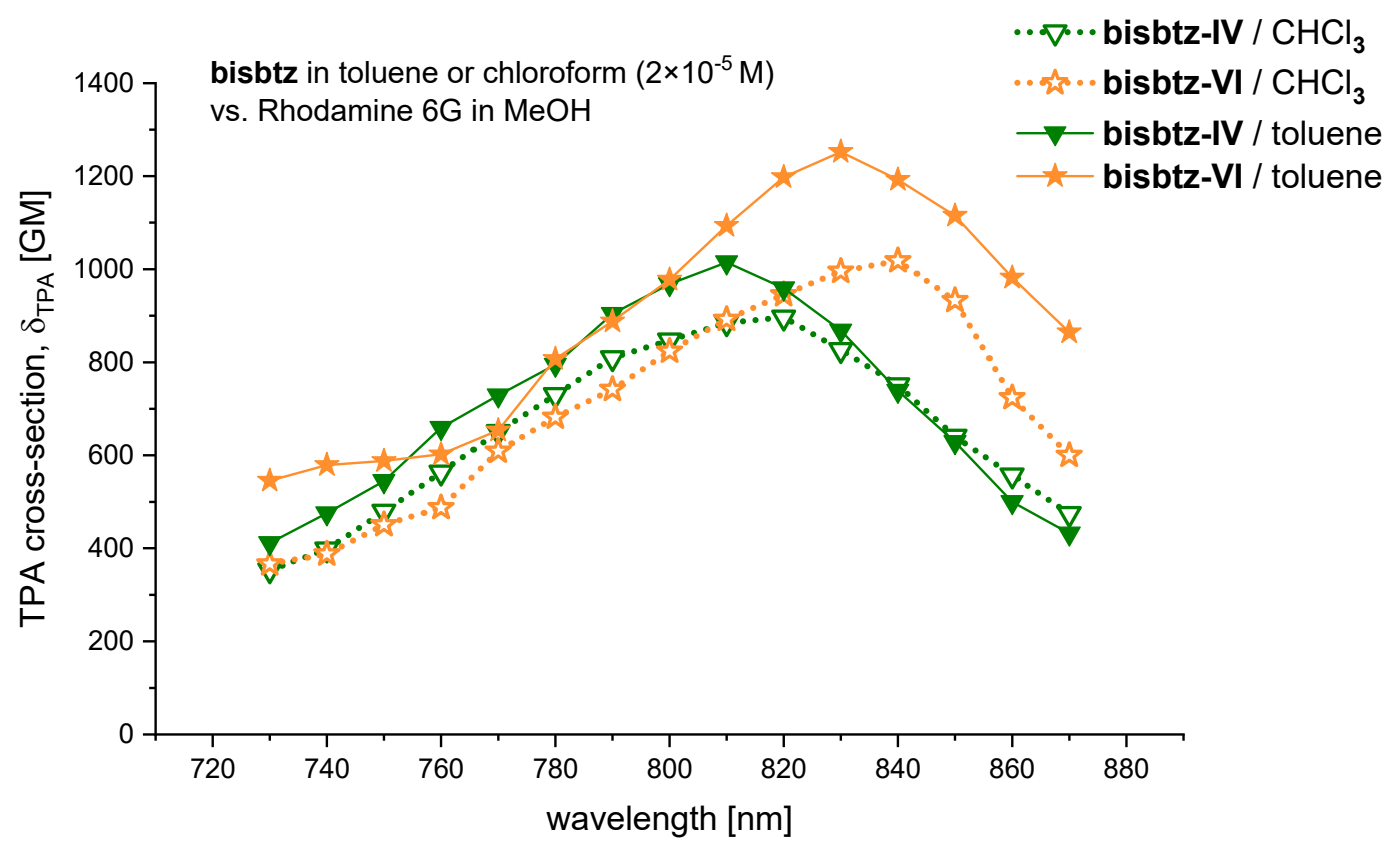

Figure S12. TPA cross-sections of bisbtz-IV and bisbtz-VI in toluene and chloroform 


\section{X-ray structure analysis}

Crystals suitable for X-ray diffraction studies were obtained by dissolving bisbtz-I in chloroform, layering it with $n$-hexane and by slow evaporation of solvents.

Single-crystal diffraction data for bisbtz-I were collected on a Bruker D8 VENTURE Kappa Duo diffractometer equipped with a PHOTON 100 CMOS detector and using monochromatic $\mathrm{Cu}-$ $\mathrm{K} \alpha$ primary radiation. The phase problem was solved by intrinsic phasing (SHELXT) ${ }^{[21]}$ and the structure model was refined by full-matrix least-squares on $\mathrm{F}^{2}$ values (SHELXL). ${ }^{[22]}$ All nonhydrogen atoms were refined anisotropically and with no restraints applied. Hydrogen atoms were put into idealized positions and were refined isotropically. Difference Fourier maps suggested the presence of some additional solvent molecule(s) in the crystal lattice, but since this solvent could not be modelled with satisfactory results, its contribution was subtracted from the data by using the SQUEEZE procedure in PLATON. ${ }^{[23]}$

CCDC 2056840 contains deposited data for the structure reported in this paper. Copies of data can be obtained free of charge from CCDC, e-mail: deposit@ccdc.cam.ac.uk.

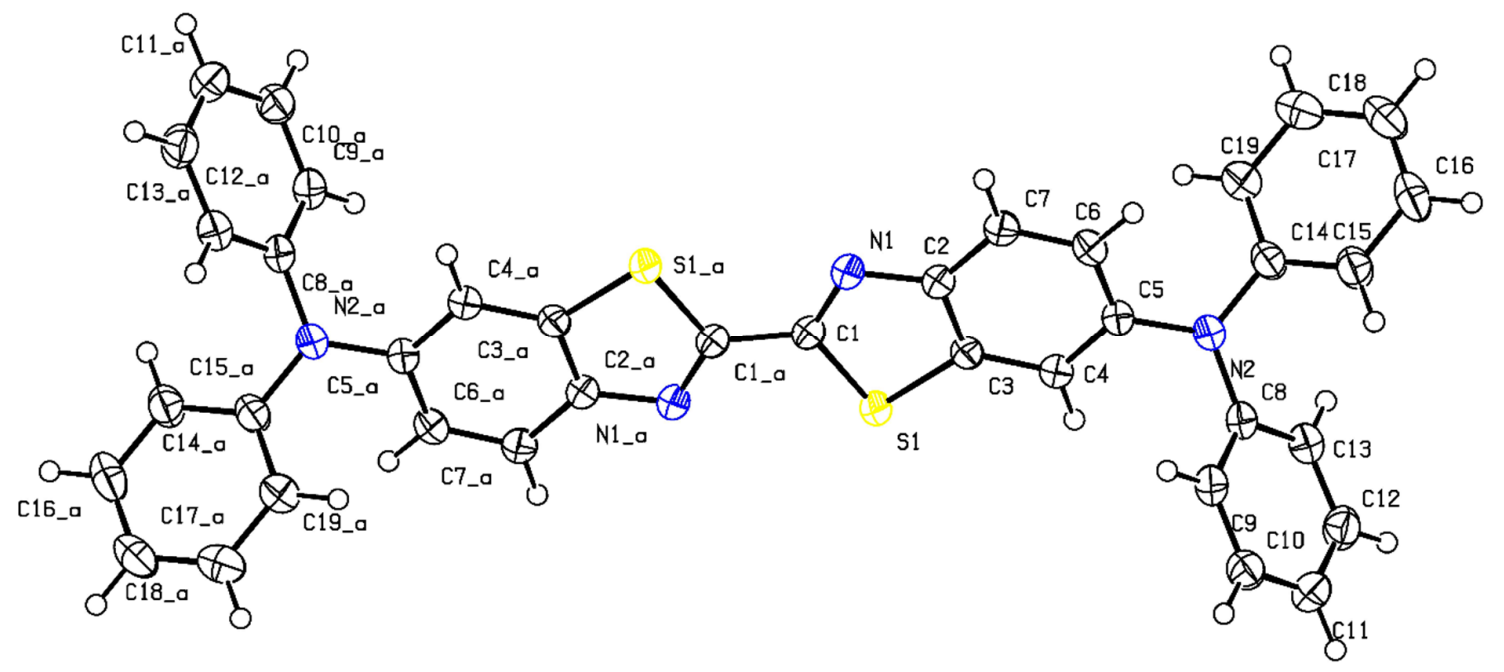

Figure S13. Molecular structure of bisbtz-I 
Table S2. Crystal data and structure refinement for bisbtz-I

CCDC No.

Empirical formula

Formula weight

Temperature

Wavelength

Crystal system, space group

Unit cell dimensions

Volume

Z, Calculated density

Absorption coefficient

$\mathrm{F}(000)$

Crystal size

Theta range for data collection

Limiting indices

Reflections collected / unique

Completeness to theta $=67.679$

Absorption correction

Max. and min. transmission

Refinement method

Data / restraints / parameters

Goodness-of-fit on $\mathrm{F}^{2}$

Final $\mathrm{R}$ indices $[\mathrm{I}>2 \operatorname{sigma}(\mathrm{I})]$

$\mathrm{R}$ indices (all data)

Extinction coefficient

Largest diff. peak and hole
2056840

$\mathrm{C}_{38} \mathrm{H}_{26} \mathrm{~N}_{4} \mathrm{~S}_{2}$

602.75

125(2) K

$1.54178 \mathrm{~A}$

Monoclinic, P2(1)/c

$\mathrm{a}=7.8308(6) \AA \quad$ alpha $=90 \mathrm{deg}$.

$\mathrm{b}=22.3973(18) \AA \quad$ beta $=93.874(3) \mathrm{deg}$.

$\mathrm{c}=10.0013(8) \AA \quad$ gamma $=90 \mathrm{deg}$.

1750.1(2) $\AA^{3}$

$2,1.144 \mathrm{~g} / \mathrm{cm}^{3}$

$1.607 \mathrm{~mm}^{-1}$

628

$0.268 \times 0.262 \times 0.127 \mathrm{~mm}$

3.947 to $72.448 \mathrm{deg}$.

$-9<=\mathrm{h}<=9,-27<=\mathrm{k}<=27,-12<=\mathrm{l}<=11$

$36144 / 3458[\mathrm{R}($ int $)=0.0319]$

$100.0 \%$

Semi-empirical from equivalents

0.82 and 0.69

Full-matrix least-squares on $\mathrm{F}^{2}$

3458 / 0 / 199

1.089

$\mathrm{R} 1=0.0348, \mathrm{wR} 2=0.0976$

$\mathrm{R} 1=0.0362, \mathrm{wR} 2=0.0992$

$\mathrm{n} / \mathrm{a}$

0.425 and -0.140 e. $\AA^{-3}$ 
Table S3. Atomic coordinates $\left(\times 10^{4}\right)$ and equivalent isotropic displacement parameters $\left(\AA^{2} \times 10^{3}\right)$ for bisbtz-I. $U(e q)$ is defined as one third of the trace of the orthogonalized $U_{i j}$ tensor.

\begin{tabular}{|c|c|c|c|c|}
\hline & $\mathrm{x}$ & $\mathrm{y}$ & $\mathrm{z}$ & $\mathrm{U}(\mathrm{eq})$ \\
\hline$C(1)$ & $533(2)$ & $4744(1)$ & $4856(1)$ & $31(1)$ \\
\hline$S(1)$ & $-428(1)$ & $4083(1)$ & $4256(1)$ & $33(1)$ \\
\hline $\mathrm{N}(1)$ & 2189(1) & $4748(1)$ & $5032(1)$ & $33(1)$ \\
\hline$C(2)$ & $2835(2)$ & $4196(1)$ & 4694(1) & $31(1)$ \\
\hline$C(3)$ & $1602(2)$ & $3771(1)$ & $4250(1)$ & $29(1)$ \\
\hline C(4) & $2045(2)$ & $3197(1)$ & $3862(1)$ & $31(1)$ \\
\hline$C(5)$ & $3775(2)$ & $3047(1)$ & $3948(1)$ & $31(1)$ \\
\hline$C(6)$ & $5011(2)$ & $3464(1)$ & $4434(1)$ & $35(1)$ \\
\hline$C(7)$ & $4569(2)$ & $4035(1)$ & $4783(2)$ & $34(1)$ \\
\hline $\mathrm{N}(2)$ & $4288(1)$ & $2474(1)$ & $3535(1)$ & $35(1)$ \\
\hline $\mathrm{C}(8)$ & $3289(2)$ & $1965(1)$ & $3822(1)$ & $33(1)$ \\
\hline$C(9)$ & $2608(2)$ & $1899(1)$ & $5071(1)$ & $35(1)$ \\
\hline$C(10)$ & $1684(2)$ & 1394(1) & $5352(1)$ & $39(1)$ \\
\hline $\mathrm{C}(11)$ & $1439(2)$ & $941(1)$ & $4413(2)$ & $43(1)$ \\
\hline$C(12)$ & $2106(2)$ & $1005(1)$ & $3170(2)$ & $42(1)$ \\
\hline$C(13)$ & $2996(2)$ & $1515(1)$ & $2865(1)$ & $37(1)$ \\
\hline$C(14)$ & $5819(2)$ & $2406(1)$ & 2859(1) & $34(1)$ \\
\hline$C(15)$ & $6795(2)$ & 1889(1) & $3035(2)$ & $40(1)$ \\
\hline$C(16)$ & $8288(2)$ & $1822(1)$ & $2374(2)$ & $48(1)$ \\
\hline$C(17)$ & $8837(2)$ & $2266(1)$ & $1557(2)$ & $47(1)$ \\
\hline $\mathrm{C}(18)$ & $7874(2)$ & $2780(1)$ & $1379(2)$ & $45(1)$ \\
\hline C(19) & $6366(2)$ & $2850(1)$ & $2014(1)$ & $39(1)$ \\
\hline
\end{tabular}

Table S4. Bond lengths $[\AA]$ and angles $[\mathrm{deg}]$ for bisbtz-I

$\begin{array}{ll}\mathrm{C}(1)-\mathrm{N}(1) & 1.2974(17) \\ \mathrm{C}(1)-\mathrm{C}(1) \# 1 & 1.456(2) \\ \mathrm{C}(1)-\mathrm{S}(1) & 1.7489(13) \\ \mathrm{S}(1)-\mathrm{C}(3) & 1.7368(12) \\ \mathrm{N}(1)-\mathrm{C}(2) & 1.3853(16) \\ \mathrm{C}(2)-\mathrm{C}(7) & 1.4021(18) \\ \mathrm{C}(2)-\mathrm{C}(3) & 1.4066(18) \\ \mathrm{C}(3)-\mathrm{C}(4) & 1.3940(17) \\ \mathrm{C}(4)-\mathrm{C}(5) & 1.3926(18) \\ \mathrm{C}(4)-\mathrm{H}(4) & 0.9500 \\ \mathrm{C}(5)-\mathrm{C}(6) & 1.4089(19) \\ \mathrm{C}(5)-\mathrm{N}(2) & 1.4147(16) \\ \mathrm{C}(6)-\mathrm{C}(7) & 1.3747(18) \\ \mathrm{C}(6)-\mathrm{H}(6) & 0.9500 \\ \mathrm{C}(7)-\mathrm{H}(7) & 0.9500 \\ \mathrm{~N}(2)-\mathrm{C}(8) & 1.4235(17)\end{array}$




$\begin{array}{ll}\mathrm{N}(2)-\mathrm{C}(14) & 1.4243(16) \\ \mathrm{C}(8)-\mathrm{C}(13) & 1.3975(19) \\ \mathrm{C}(8)-\mathrm{C}(9) & 1.3981(19) \\ \mathrm{C}(9)-\mathrm{C}(10) & 1.381(2) \\ \mathrm{C}(9)-\mathrm{H}(9) & 0.9500 \\ \mathrm{C}(10)-\mathrm{C}(11) & 1.388(2) \\ \mathrm{C}(10)-\mathrm{H}(10) & 0.9500 \\ \mathrm{C}(11)-\mathrm{C}(12) & 1.388(2) \\ \mathrm{C}(11)-\mathrm{H}(11) & 0.9500 \\ \mathrm{C}(12)-\mathrm{C}(13) & 1.384(2) \\ \mathrm{C}(12)-\mathrm{H}(12) & 0.9500 \\ \mathrm{C}(13)-\mathrm{H}(13) & 0.9500 \\ \mathrm{C}(14)-\mathrm{C}(15) & 1.3915(19) \\ \mathrm{C}(14)-\mathrm{C}(19) & 1.392(2) \\ \mathrm{C}(15)-\mathrm{C}(16) & 1.389(2) \\ \mathrm{C}(15)-\mathrm{H}(15) & 0.9500 \\ \mathrm{C}(16)-\mathrm{C}(17) & 1.374(2) \\ \mathrm{C}(16)-\mathrm{H}(16) & 0.9500 \\ \mathrm{C}(17)-\mathrm{C}(18) & 1.381(2) \\ \mathrm{C}(17)-\mathrm{H}(17) & 0.9500 \\ \mathrm{C}(18)-\mathrm{C}(19) & 1.387(2) \\ \mathrm{C}(18)-\mathrm{H}(18) & 0.9500 \\ \mathrm{C}(19)-\mathrm{H}(19) & 0.9500 \\ & \\ \mathrm{~N}(1)-\mathrm{C}(1)-\mathrm{C}(1) \# 1 & 123.37(15) \\ \mathrm{N}(1)-\mathrm{C}(1)-\mathrm{S}(1) & 117.00(9) \\ \mathrm{C}(1) \# 1-\mathrm{C}(1)-\mathrm{S}(1) & 119.63(13) \\ \mathrm{C}(3)-\mathrm{S}(1)-\mathrm{C}(1) & 88.26(6) \\ \mathrm{C}(1)-\mathrm{N}(1)-\mathrm{C}(2) & 109.90(11) \\ \mathrm{N}(1)-\mathrm{C}(2)-\mathrm{C}(7) & 125.63(12) \\ \mathrm{N}(1)-\mathrm{C}(2)-\mathrm{C}(3) & 115.22(11) \\ \mathrm{C}(7)-\mathrm{C}(2)-\mathrm{C}(3) & 119.14(11) \\ \mathrm{C}(4)-\mathrm{C}(3)-\mathrm{C}(2) & 122.25(11) \\ \mathrm{C}(4)-\mathrm{C}(3)-\mathrm{S}(1) & 128.13(10) \\ \mathrm{C}(2)-\mathrm{C}(3)-\mathrm{S}(1) & 109.61(9) \\ \mathrm{C}(5)-\mathrm{C}(4)-\mathrm{C}(3) & 117.71(12) \\ \mathrm{C}(5)-\mathrm{C}(4)-\mathrm{H}(4) & 121.1 \\ \mathrm{C}(3)-\mathrm{C}(4)-\mathrm{H}(4) & 121.1 \\ \mathrm{C}(4)-\mathrm{C}(5)-\mathrm{C}(6) & 120.27(12) \\ \mathrm{C}(4)-\mathrm{C}(5)-\mathrm{N}(2) & 119.67(12) \\ \mathrm{C}(6)-\mathrm{C}(5)-\mathrm{N}(2) & 120.06(11) \\ \mathrm{C}(7)-\mathrm{C}(6)-\mathrm{C}(5) & 121.69(12) \\ \mathrm{C}(7)-\mathrm{C}(6)-\mathrm{H}(6) & 119.2 \\ \mathrm{C}(5)-\mathrm{C}(6)-\mathrm{H}(6) & 119.2 \\ \mathrm{C}(6)-\mathrm{C}(7)-\mathrm{C}(2) & 118.88(12) \\ \mathrm{C}(6)-\mathrm{C}(7)-\mathrm{H}(7) & 120.6 \\ \mathrm{C}(2)-\mathrm{C}(7)-\mathrm{H}(7) & 120.6 \\ \mathrm{C}(5)-\mathrm{N}(2)-\mathrm{C}(8) & 119.80(10) \\ \mathrm{C}(5)-\mathrm{N}(2)-\mathrm{C}(14) & 120.07(11) \\ \mathrm{C}(8)-\mathrm{N}(2)-\mathrm{C}(14) & 120.12(11) \\ \mathrm{C}(13)-\mathrm{C}(8)-\mathrm{C}(9) & 118) \\ & \end{array}$




$\begin{array}{ll}\mathrm{C}(13)-\mathrm{C}(8)-\mathrm{N}(2) & 120.36(12) \\ \mathrm{C}(9)-\mathrm{C}(8)-\mathrm{N}(2) & 120.85(12) \\ \mathrm{C}(10)-\mathrm{C}(9)-\mathrm{C}(8) & 120.33(13) \\ \mathrm{C}(10)-\mathrm{C}(9)-\mathrm{H}(9) & 119.8 \\ \mathrm{C}(8)-\mathrm{C}(9)-\mathrm{H}(9) & 119.8 \\ \mathrm{C}(9)-\mathrm{C}(10)-\mathrm{C}(11) & 120.72(13) \\ \mathrm{C}(9)-\mathrm{C}(10)-\mathrm{H}(10) & 119.6 \\ \mathrm{C}(11)-\mathrm{C}(10)-\mathrm{H}(10) & 119.6 \\ \mathrm{C}(10)-\mathrm{C}(11)-\mathrm{C}(12) & 119.20(13) \\ \mathrm{C}(10)-\mathrm{C}(11)-\mathrm{H}(11) & 120.4 \\ \mathrm{C}(12)-\mathrm{C}(11)-\mathrm{H}(11) & 120.4 \\ \mathrm{C}(13)-\mathrm{C}(12)-\mathrm{C}(11) & 120.57(13) \\ \mathrm{C}(13)-\mathrm{C}(12)-\mathrm{H}(12) & 119.7 \\ \mathrm{C}(11)-\mathrm{C}(12)-\mathrm{H}(12) & 119.7 \\ \mathrm{C}(12)-\mathrm{C}(13)-\mathrm{C}(8) & 120.35(13) \\ \mathrm{C}(12)-\mathrm{C}(13)-\mathrm{H}(13) & 119.8 \\ \mathrm{C}(8)-\mathrm{C}(13)-\mathrm{H}(13) & 119.8 \\ \mathrm{C}(15)-\mathrm{C}(14)-\mathrm{C}(19) & 118.90(13) \\ \mathrm{C}(15)-\mathrm{C}(14)-\mathrm{N}(2) & 120.14(13) \\ \mathrm{C}(19)-\mathrm{C}(14)-\mathrm{N}(2) & 120.96(12) \\ \mathrm{C}(16)-\mathrm{C}(15)-\mathrm{C}(14) & 120.08(15) \\ \mathrm{C}(16)-\mathrm{C}(15)-\mathrm{H}(15) & 120.0 \\ \mathrm{C}(14)-\mathrm{C}(15)-\mathrm{H}(15) & 120.0 \\ \mathrm{C}(17)-\mathrm{C}(16)-\mathrm{C}(15) & 120.85(15) \\ \mathrm{C}(17)-\mathrm{C}(16)-\mathrm{H}(16) & 119.6 \\ \mathrm{C}(15)-\mathrm{C}(16)-\mathrm{H}(16) & 119.6 \\ \mathrm{C}(16)-\mathrm{C}(17)-\mathrm{C}(18) & 119.32(13) \\ \mathrm{C}(16)-\mathrm{C}(17)-\mathrm{H}(17) & 120.3 \\ \mathrm{C}(18)-\mathrm{C}(17)-\mathrm{H}(17) & 120.3 \\ \mathrm{C}(17)-\mathrm{C}(18)-\mathrm{C}(19) & 120.65(15) \\ \mathrm{C}(17)-\mathrm{C}(18)-\mathrm{H}(18) & 119.7 \\ \mathrm{C}(19)-\mathrm{C}(18)-\mathrm{H}(18) & 119.7 \\ \mathrm{C}(18)-\mathrm{C}(19)-\mathrm{C}(14) & 120.19(14) \\ \mathrm{C}(18)-\mathrm{C}(19)-\mathrm{H}(19) & 119.9 \\ \mathrm{C}(14)-\mathrm{C}(19)-\mathrm{H}(19) & 119.9 \\ & \\ & \\ & \end{array}$

Symmetry transformations used to generate equivalent atoms:

$\# 1-\mathrm{x},-\mathrm{y}+1,-\mathrm{z}+1$ 
Table S5. Anisotropic displacement parameters $\left(\mathrm{A}^{\wedge} 2 \times 10^{\wedge} 3\right)$ for bisbtz-I. The anisotropic displacement factor exponent takes the form: $-2 \mathrm{pi}^{\wedge} 2\left[\mathrm{~h}^{\wedge} 2 \mathrm{a}^{* \wedge} 2 \mathrm{U} 11+\ldots+2 \mathrm{~h} \mathrm{k} \mathrm{a} \mathrm{a}^{*} \mathrm{U}\right.$ 12]

\begin{tabular}{lcccccc}
\hline & $\mathrm{U} 11$ & $\mathrm{U} 22$ & $\mathrm{U} 33$ & $\mathrm{U} 23$ & $\mathrm{U} 13$ & $\mathrm{U} 12$ \\
\hline & & & & & & \\
$\mathrm{C}(1)$ & $32(1)$ & $25(1)$ & $37(1)$ & $5(1)$ & $7(1)$ & $2(1)$ \\
$\mathrm{S}(1)$ & $25(1)$ & $26(1)$ & $48(1)$ & $0(1)$ & $6(1)$ & $3(1)$ \\
$\mathrm{N}(1)$ & $31(1)$ & $25(1)$ & $42(1)$ & $2(1)$ & $7(1)$ & $2(1)$ \\
$\mathrm{C}(2)$ & $30(1)$ & $25(1)$ & $37(1)$ & $3(1)$ & $7(1)$ & $1(1)$ \\
$\mathrm{C}(3)$ & $26(1)$ & $28(1)$ & $35(1)$ & $4(1)$ & $6(1)$ & $2(1)$ \\
$\mathrm{C}(4)$ & $28(1)$ & $27(1)$ & $38(1)$ & $0(1)$ & $6(1)$ & $0(1)$ \\
$\mathrm{C}(5)$ & $31(1)$ & $26(1)$ & $38(1)$ & $0(1)$ & $8(1)$ & $4(1)$ \\
$\mathrm{C}(6)$ & $26(1)$ & $33(1)$ & $46(1)$ & $0(1)$ & $6(1)$ & $3(1)$ \\
$\mathrm{C}(7)$ & $28(1)$ & $29(1)$ & $46(1)$ & $-1(1)$ & $4(1)$ & $-1(1)$ \\
$\mathrm{N}(2)$ & $31(1)$ & $28(1)$ & $47(1)$ & $-4(1)$ & $12(1)$ & $3(1)$ \\
$\mathrm{C}(8)$ & $29(1)$ & $27(1)$ & $42(1)$ & $-1(1)$ & $6(1)$ & $6(1)$ \\
$\mathrm{C}(9)$ & $35(1)$ & $31(1)$ & $40(1)$ & $-2(1)$ & $4(1)$ & $6(1)$ \\
$\mathrm{C}(10)$ & $40(1)$ & $38(1)$ & $40(1)$ & $3(1)$ & $9(1)$ & $4(1)$ \\
$\mathrm{C}(11)$ & $43(1)$ & $31(1)$ & $55(1)$ & $3(1)$ & $6(1)$ & $-1(1)$ \\
$\mathrm{C}(12)$ & $42(1)$ & $34(1)$ & $50(1)$ & $-7(1)$ & $2(1)$ & $2(1)$ \\
$\mathrm{C}(13)$ & $36(1)$ & $35(1)$ & $40(1)$ & $-3(1)$ & $7(1)$ & $4(1)$ \\
$\mathrm{C}(14)$ & $28(1)$ & $38(1)$ & $37(1)$ & $-5(1)$ & $6(1)$ & $3(1)$ \\
$\mathrm{C}(15)$ & $35(1)$ & $40(1)$ & $45(1)$ & $-3(1)$ & $6(1)$ & $7(1)$ \\
$\mathrm{C}(16)$ & $36(1)$ & $53(1)$ & $54(1)$ & $-12(1)$ & $5(1)$ & $14(1)$ \\
$\mathrm{C}(17)$ & $31(1)$ & $63(1)$ & $50(1)$ & $-15(1)$ & $11(1)$ & $0(1)$ \\
$\mathrm{C}(18)$ & $38(1)$ & $57(1)$ & $42(1)$ & $-3(1)$ & $8(1)$ & $-9(1)$ \\
$\mathrm{C}(19)$ & $33(1)$ & $41(1)$ & $43(1)$ & $0(1)$ & $5(1)$ & $1(1)$ \\
& & & & & & \\
\hline
\end{tabular}

Table S6. Hydrogen coordinates $\left(\times 10^{4}\right)$ and isotropic displacement parameters $\left(\AA^{2} \times 10^{3}\right)$ for bisbtz-I

\begin{tabular}{lcccl}
\hline & $x$ & $y$ & $z$ & $U(e q)$ \\
\hline$H(4)$ & 1196 & 2918 & 3549 & 37 \\
$\mathrm{H}(6)$ & 6181 & 3349 & 4523 & 42 \\
$\mathrm{H}(7)$ & 5424 & 4315 & 5079 & 41 \\
$\mathrm{H}(9)$ & 2781 & 2203 & 5728 & 42 \\
$\mathrm{H}(10)$ & 1211 & 1357 & 6199 & 47 \\
$\mathrm{H}(11)$ & 822 & 591 & 4618 & 52 \\
$\mathrm{H}(12)$ & 1950 & 695 & 2524 & 51 \\
$\mathrm{H}(13)$ & 3411 & 1560 & 2000 & 44 \\
$\mathrm{H}(15)$ & 6440 & 1581 & 3608 & 48 \\
$\mathrm{H}(16)$ & 8937 & 1465 & 2488 & 57 \\
$\mathrm{H}(17)$ & 9869 & 2220 & 1120 & 57 \\
$\mathrm{H}(18)$ & 8248 & 3088 & 816 & 55 \\
$\mathrm{H}(19)$ & 5705 & 3203 & 1873 & 47 \\
& & & & \\
\hline
\end{tabular}


Table S7. Torsion angles [deg] for bisbtz-I

\begin{tabular}{|c|c|}
\hline $\mathrm{N}(1)-\mathrm{C}(1)-\mathrm{S}(1)-\mathrm{C}(3)$ & $0.57(11)$ \\
\hline $\mathrm{C}(1) \# 1-\mathrm{C}(1)-\mathrm{S}(1)-\mathrm{C}(3)$ & $-178.92(14)$ \\
\hline $\mathrm{C}(1) \# 1-\mathrm{C}(1)-\mathrm{N}(1)-\mathrm{C}(2)$ & $178.96(15)$ \\
\hline $\mathrm{S}(1)-\mathrm{C}(1)-\mathrm{N}(1)-\mathrm{C}(2)$ & $-0.51(14)$ \\
\hline $\mathrm{C}(1)-\mathrm{N}(1)-\mathrm{C}(2)-\mathrm{C}(7)$ & $-178.75(13)$ \\
\hline $\mathrm{C}(1)-\mathrm{N}(1)-\mathrm{C}(2)-\mathrm{C}(3)$ & $0.14(16)$ \\
\hline $\mathrm{N}(1)-\mathrm{C}(2)-\mathrm{C}(3)-\mathrm{C}(4)$ & $179.40(11)$ \\
\hline $\mathrm{C}(7)-\mathrm{C}(2)-\mathrm{C}(3)-\mathrm{C}(4)$ & $-1.63(19)$ \\
\hline $\mathrm{N}(1)-\mathrm{C}(2)-\mathrm{C}(3)-\mathrm{S}(1)$ & $0.27(14)$ \\
\hline $\mathrm{C}(7)-\mathrm{C}(2)-\mathrm{C}(3)-\mathrm{S}(1)$ & $179.24(10)$ \\
\hline $\mathrm{C}(1)-\mathrm{S}(1)-\mathrm{C}(3)-\mathrm{C}(4)$ & $-179.50(12)$ \\
\hline $\mathrm{C}(1)-\mathrm{S}(1)-\mathrm{C}(3)-\mathrm{C}(2)$ & $-0.43(10)$ \\
\hline$C(2)-C(3)-C(4)-C(5)$ & $1.17(19)$ \\
\hline $\mathrm{S}(1)-\mathrm{C}(3)-\mathrm{C}(4)-\mathrm{C}(5)$ & $-179.88(10)$ \\
\hline$C(3)-C(4)-C(5)-C(6)$ & $0.96(19)$ \\
\hline $\mathrm{C}(3)-\mathrm{C}(4)-\mathrm{C}(5)-\mathrm{N}(2)$ & $-178.51(11)$ \\
\hline $\mathrm{C}(4)-\mathrm{C}(5)-\mathrm{C}(6)-\mathrm{C}(7)$ & $-2.7(2)$ \\
\hline $\mathrm{N}(2)-\mathrm{C}(5)-\mathrm{C}(6)-\mathrm{C}(7)$ & $176.77(13)$ \\
\hline$C(5)-C(6)-C(7)-C(2)$ & $2.2(2)$ \\
\hline $\mathrm{N}(1)-\mathrm{C}(2)-\mathrm{C}(7)-\mathrm{C}(6)$ & $178.77(13)$ \\
\hline $\mathrm{C}(3)-\mathrm{C}(2)-\mathrm{C}(7)-\mathrm{C}(6)$ & $-0.1(2)$ \\
\hline $\mathrm{C}(4)-\mathrm{C}(5)-\mathrm{N}(2)-\mathrm{C}(8)$ & $-39.05(18)$ \\
\hline $\mathrm{C}(6)-\mathrm{C}(5)-\mathrm{N}(2)-\mathrm{C}(8)$ & $141.47(13)$ \\
\hline $\mathrm{C}(4)-\mathrm{C}(5)-\mathrm{N}(2)-\mathrm{C}(14)$ & $142.32(13)$ \\
\hline $\mathrm{C}(6)-\mathrm{C}(5)-\mathrm{N}(2)-\mathrm{C}(14)$ & $-37.15(19)$ \\
\hline $\mathrm{C}(5)-\mathrm{N}(2)-\mathrm{C}(8)-\mathrm{C}(13)$ & $139.09(13)$ \\
\hline $\mathrm{C}(14)-\mathrm{N}(2)-\mathrm{C}(8)-\mathrm{C}(13)$ & $-42.29(19)$ \\
\hline $\mathrm{C}(5)-\mathrm{N}(2)-\mathrm{C}(8)-\mathrm{C}(9)$ & $-42.03(18)$ \\
\hline $\mathrm{C}(14)-\mathrm{N}(2)-\mathrm{C}(8)-\mathrm{C}(9)$ & $136.60(13)$ \\
\hline $\mathrm{C}(13)-\mathrm{C}(8)-\mathrm{C}(9)-\mathrm{C}(10)$ & $0.9(2)$ \\
\hline $\mathrm{N}(2)-\mathrm{C}(8)-\mathrm{C}(9)-\mathrm{C}(10)$ & $-178.03(12)$ \\
\hline $\mathrm{C}(8)-\mathrm{C}(9)-\mathrm{C}(10)-\mathrm{C}(11)$ & $1.0(2)$ \\
\hline $\mathrm{C}(9)-\mathrm{C}(10)-\mathrm{C}(11)-\mathrm{C}(12)$ & $-1.3(2)$ \\
\hline $\mathrm{C}(10)-\mathrm{C}(11)-\mathrm{C}(12)-\mathrm{C}(13)$ & $-0.4(2)$ \\
\hline $\mathrm{C}(11)-\mathrm{C}(12)-\mathrm{C}(13)-\mathrm{C}(8)$ & $2.3(2)$ \\
\hline $\mathrm{C}(9)-\mathrm{C}(8)-\mathrm{C}(13)-\mathrm{C}(12)$ & $-2.5(2)$ \\
\hline $\mathrm{N}(2)-\mathrm{C}(8)-\mathrm{C}(13)-\mathrm{C}(12)$ & $176.40(12)$ \\
\hline $\mathrm{C}(5)-\mathrm{N}(2)-\mathrm{C}(14)-\mathrm{C}(15)$ & $147.35(13)$ \\
\hline $\mathrm{C}(8)-\mathrm{N}(2)-\mathrm{C}(14)-\mathrm{C}(15)$ & $-31.26(19)$ \\
\hline $\mathrm{C}(5)-\mathrm{N}(2)-\mathrm{C}(14)-\mathrm{C}(19)$ & $-32.68(19)$ \\
\hline $\mathrm{C}(8)-\mathrm{N}(2)-\mathrm{C}(14)-\mathrm{C}(19)$ & $148.70(13)$ \\
\hline $\mathrm{C}(19)-\mathrm{C}(14)-\mathrm{C}(15)-\mathrm{C}(16)$ & $0.0(2)$ \\
\hline $\mathrm{N}(2)-\mathrm{C}(14)-\mathrm{C}(15)-\mathrm{C}(16)$ & 179.93(13) \\
\hline C(14)-C(15)-C(16)-C(17) & $1.0(2)$ \\
\hline $\mathrm{C}(15)-\mathrm{C}(16)-\mathrm{C}(17)-\mathrm{C}(18)$ & $-1.0(2)$ \\
\hline $\mathrm{C}(16)-\mathrm{C}(17)-\mathrm{C}(18)-\mathrm{C}(19)$ & $0.0(2)$ \\
\hline $\mathrm{C}(17)-\mathrm{C}(18)-\mathrm{C}(19)-\mathrm{C}(14)$ & $1.0(2)$ \\
\hline $\mathrm{C}(15)-\mathrm{C}(14)-\mathrm{C}(19)-\mathrm{C}(18)$ & $-1.0(2)$ \\
\hline $\mathrm{N}(2)-\mathrm{C}(14)-\mathrm{C}(19)-\mathrm{C}(18)$ & 179.07(13) \\
\hline
\end{tabular}

Symmetry transformations used to generate equivalent atoms:

$\# 1-\mathrm{x},-\mathrm{y}+1,-\mathrm{z}+1$ 


\section{Computational details and results}

The ground-state structures of all systems under investigation were fully optimized at the $\mathrm{PBE0}^{[24],[25]}$ level of theory, employing all-electron def2-TZVP basis set ${ }^{[26]}$ an atom-pairwise correction for dispersion forces via Grimme's D3 model ${ }^{[27]}$ with Becke-Johnson (BJ) ${ }^{[28]}$ damping, in the Turbomole program. ${ }^{[29]}$ All structures were characterized as true minima on the harmonic potential energy hypersurface through vibrational analysis (no imaginary frequencies were found).

The two-component relativistic all-electron DFT calculations of the NMR nuclear shieldings ${ }^{[30],[31]}$ were performed using the Amsterdam Density Functional (ADF) ${ }^{[32]}$ program suite, ${ }^{[33]}$ employing the global hydrid PBE0 (with 25\% exact-exchange admixture) ${ }^{[25]}$ exchangecorrelation functional, in conjunction with Slater-type orbital basis sets of triple- $\zeta$ doubly polarized (TZ2P) quality and an integration accuracy of 5.0. The relativistic zeroth-order regular approximation (ZORA) ${ }^{[30],[31],[33]}$ calculations of NMR shieldings were done by using gaugeincluding atomic orbitals (GIAOs). ${ }^{[34]}$ The computed ${ }^{15} \mathrm{~N}$ and ${ }^{1} \mathrm{H}$ nuclear shieldings were converted to chemical shifts $(\delta$, in ppm) relative to the shielding of nitromethane and tetramethylsilane (TMS), respectively.

Time-dependent DFT method using the Coulomb-attenuated CAM-B3LYP ${ }^{[35]}$ exchangecorrelation functional, respectively, in conjunction with the $6-311++\mathrm{G}^{* *}$ basis set was applied to get vertical excitation energies $\left(E_{n}\right)$, transition dipole moments $\left(\mu_{0}\right)$ and adiabatic dipole moment changes $\left(\Delta \mu_{n}\right)$ between the ground-state and the $n$-th excited state. The excited-to-excited transition dipole moments $\mu$ n were obtained from the double-residue of the quadratic response function. All these calculations were carried out with the Dalton program. ${ }^{[36]}$

The TPA cross-sections $\delta$ TPA were evaluated by calculating the two-photon transition moment matrix elements $S_{\alpha \beta}$ in the Dalton program. The matrix elements $S_{\alpha \beta}$ for the two-photon resonant absorption of identical energy can be identified from the sum-over-states formula a 
$S_{\alpha \beta}=\sum_{n}\left[\frac{\left\langle 0\left|\mu_{\alpha}\right| n\right\rangle\left\langle n\left|\mu_{\beta}\right| f\right\rangle}{\omega_{n}-\frac{\omega_{f}}{2}}+\frac{\left\langle 0\left|\mu_{\beta}\right| n\right\rangle\left\langle n\left|\mu_{\alpha}\right| f\right\rangle}{\omega_{n}-\frac{\omega_{f}}{2}}\right] \quad(\alpha, \beta=x, y, z)$

where $\omega_{n}$ represents the excitation energy from the ground state $|0\rangle$ to the excited state $|n\rangle, \omega_{f} / 2$ corresponds to half of the excitation energy associated with the transition from the ground to the final excited state $|f\rangle, \mu_{\alpha}$ and $\mu_{\beta}$ are the Cartesian components of the electronic dipole moment operator. The explicit summation over all excited states of the molecule was avoided through the use of quadratic response theory. Within this formalism, matrix elements $S_{\alpha \beta}$ are extracted as a single residue of the appropriate quadratic response function for the dipole moment operators. This approach is described in detail in refs. [37], [38] and [39].

TPA cross-sections of molecules for linearly polarized monochromatic light were calculated (in atomic units) from $S_{\alpha \beta}$ elements as:

$$
\delta_{a . u .}=\frac{1}{30} \sum_{\alpha, \beta}\left(2 S_{\alpha \alpha} S_{\beta \beta}^{*}+4 S_{\alpha \beta} S_{\beta \alpha}^{*}\right)
$$

Finally, $\delta_{\text {a.u. }}$ values were converted to TPA cross-sections $\delta_{\mathrm{TPA}}$, which are directly comparable to experiment, using the following relationship:

$$
\delta_{T P A}=\frac{(2 \pi)^{3} \alpha a_{0}^{5}}{c} \frac{\omega^{2}}{\pi \Gamma} \delta_{a . u}
$$

Here, $\alpha$ is the fine structure constant, $a_{0}$ the Bohr radius, $c$ the speed of light, $\omega$ the energy of the exciting photon (in case of the TPA process, one half of the excitation energy) and $\pi \Gamma$ a normalization factor due to the Lorentzian line-shape broadening of the excited state $(\Gamma=0.2 \mathrm{eV}$ was used throughout this work). The units of $\delta$ TPA will become GM $\left(\mathrm{cm}^{4}\right.$.s.photon $\left.{ }^{-1}\right)$, provided we use centimeter-gram-second units for $a_{0}$ and $c$, and atomic units for $\omega$ and $\Gamma$. Note that effects on nuclear motion, which may also contribute to $\delta_{\text {TPA, }}$ are not included in our treatment. 
Table S8. Calculated one-photon absorption parameters and TPA cross-sections for the first 3 excitations at the CAM-B3LYP/6-311++G** level of theory (in vacuo) for pertinent push-pull 2H-benzothiazole (btz-VI), quadrupolar 2,2'-bibenzothiazole (bisbtz-VI) and its 2,2'-bipyridinecored congener (see Computational details). ${ }^{a}$

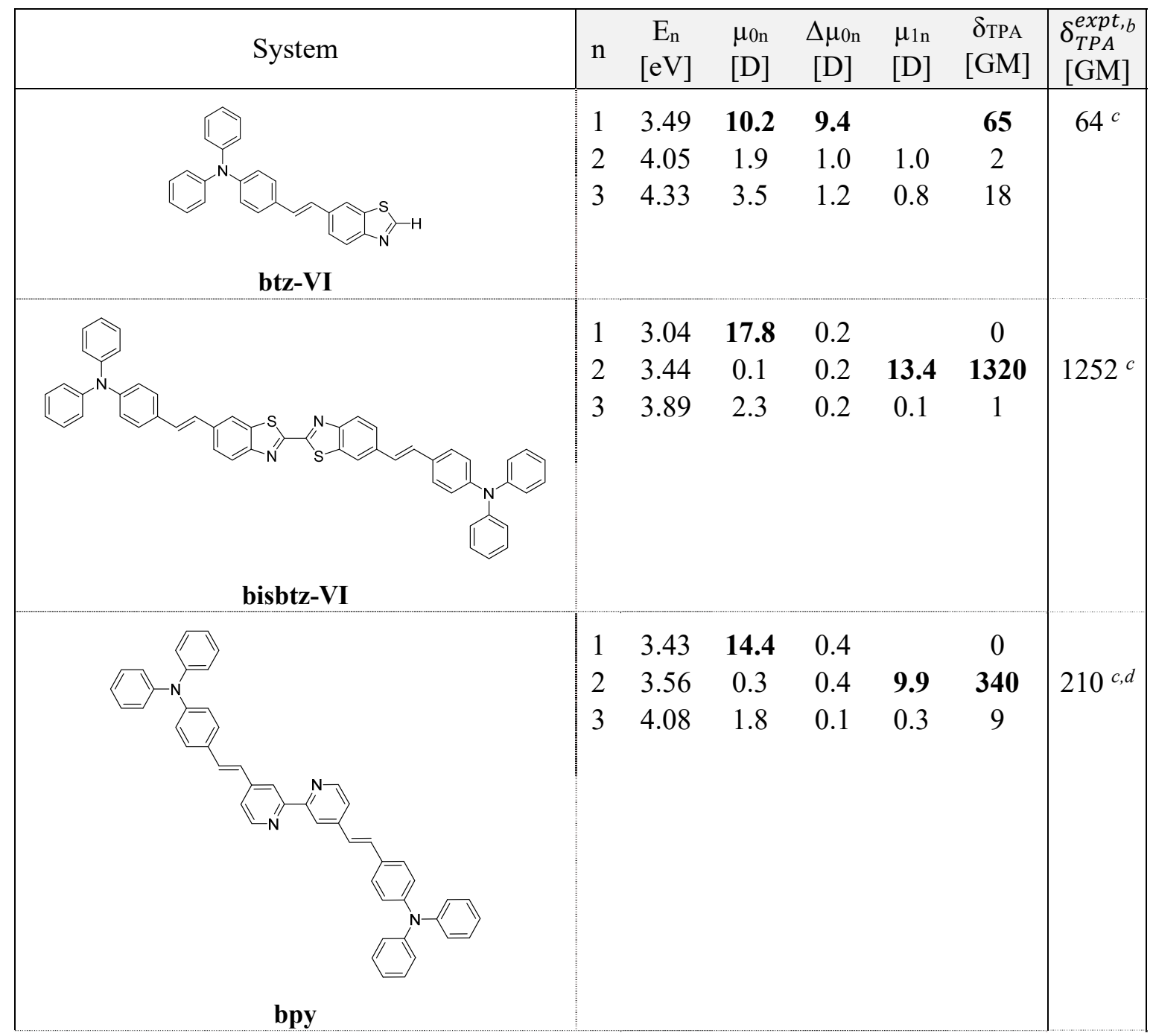

${ }^{a}$ Only s-trans configuration considered in calculations of biheteroaryls. Since s-cis conformers are energetically disfavored by ca. $24 \mathrm{~kJ} / \mathrm{mol}$, they give rise to a negligible Boltzmann weighting factor. ${ }^{b}$ Experimental TPA cross-sections at their resonant maxima. ${ }^{c}$ This work (see Figure S11 in SI). ${ }^{d}$ In ref. [40], the maximum TPA cross-section for bpy in chloroform is reported as high as $219 \mathrm{GM}$ at $690 \mathrm{~nm}$. 
Table S9. Calculated one-photon absorption parameters and TPA cross-sections for the first three excitations at the CAM-B3LYP/6-311++G** level of theory (see Computational details). ${ }^{a}$

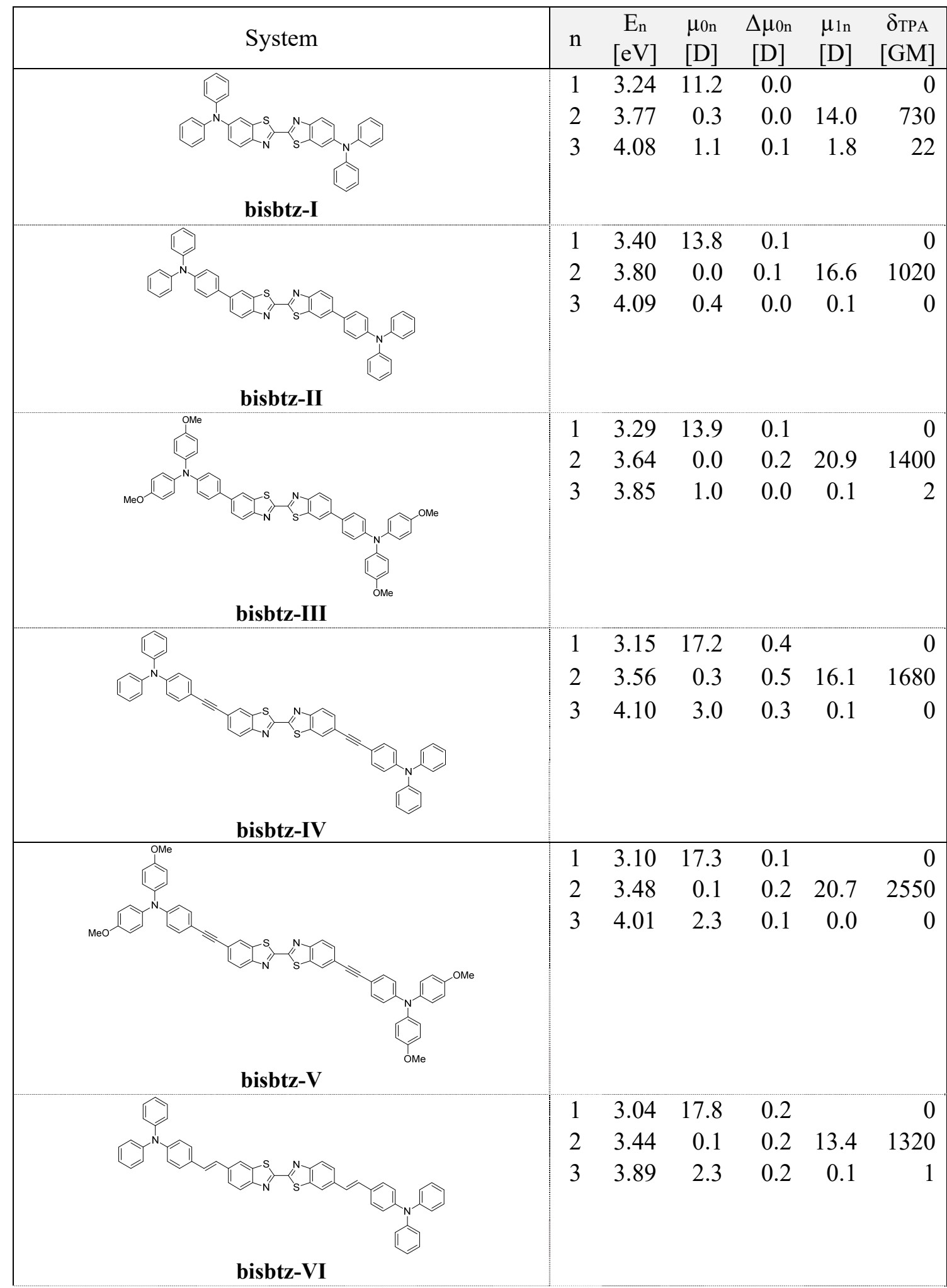

${ }^{a}$ Only s-trans configuration considered in the calculations. Since $s$-cis conformers are energetically disfavored by ca. $24 \mathrm{~kJ} / \mathrm{mol}$, they give rise to a negligible Boltzmann weighting factor. 
Considering only the first $(n=1)$ or first two $(n=1,2)$ excited states in the summation of Eq. S1 above and taking Eqs. S2 and S3 into account, one can derive the following two- and three-state models for TPA cross-sections:

Two-state model for dipoles

$$
\delta_{T P A}^{2 S M}\left(S_{0} \rightarrow S_{1}\right)=\frac{8 \pi^{2} \alpha a_{0}^{5}}{15 c} \frac{1}{\Gamma}(\underbrace{4\left|\mu_{01}\right|^{2}\left|\Delta \mu_{01}\right|^{2}\left(1+2 \cos ^{2} \varphi\right)}_{\text {two-state "dipolar"term }})
$$

\section{Three-state model for (quasi-)quadrupoles}

$$
\delta_{T P A}^{3 S M}\left(S_{0} \rightarrow S_{2}\right)=\frac{8 \pi^{2} \alpha a_{0}^{5}}{15 c} \frac{1}{\Gamma}(\underbrace{E_{2}^{2} \frac{\left|\mu_{01}\right|^{2}\left|\mu_{12}\right|^{2}}{\left(E_{1}-\frac{E_{2}}{2}\right)^{2}}\left(1+2 \cos ^{2} \theta\right)}_{\text {three-state term }}+\underbrace{4\left|\mu_{02}\right|^{2}\left|\Delta \mu_{02}\right|^{2}\left(1+2 \cos ^{2} \varphi\right)}_{\text {two-state "dipolar"term }})
$$

In true quadrupoles the dipolar terms diminishe due to $\Delta \mu_{0 \mathrm{n}}=0 . \varphi$ is the angle between the vectors $\mu_{0 n}$ and $\Delta \mu_{0 n}$, and $\theta$ is the angle between the vectors $\mu_{01}$ and $\mu_{12}$. In all systems under investigation, the corresponding vectors are oriented almost parallel or anti-parallel with respect to each other, giving constant factors $\left(1+2 \cos ^{2} \theta\right) \approx 3$ and $\left(1+2 \cos ^{2} \varphi\right) \approx 3$.

Table S10. Analysis of TPA cross-sections in pertinent systems relevant to this work

\begin{tabular}{lcccccc}
\hline System & $\begin{array}{c}\left|\mu_{01}\right| \\
{[\mathrm{D}]}\end{array}$ & $\begin{array}{c}\left|\Delta \mu_{01}\right| \\
{[\mathrm{D}]}\end{array}$ & $\begin{array}{c}\left|\mu_{12}\right| \\
{[\mathrm{D}]}\end{array}$ & $\begin{array}{c}E_{1}-E_{2} / 2 \\
{[\mathrm{eV}]}\end{array}$ & $\begin{array}{c}\delta_{T P A}^{S M} \\
{[\mathrm{GM}]}\end{array}$ & $\begin{array}{c}\delta_{T P A}^{\text {full }} \\
{[\mathrm{GM}]}\end{array}$ \\
\hline btz-VI & 10.2 & 9.4 & 1.0 & 1.47 & 189 & 65 \\
\hline bisbtz-VI & 17.8 & 0.2 & 13.4 & 1.32 & 2010 & 1320 \\
bpy & 14.4 & 0.4 & 9.9 & 1.65 & 494 & 340 \\
\hline
\end{tabular}

TPA cross-sections estimated from the two- or three-state model follow the trends obtained from response theory $\left(\delta_{T P A}^{f u l l}\right)$, justifying the use of these simplified models for more detailed analysis. Hence, the TPA superiority of bisbtz-VI can be ascribed to the highest transition dipole moments $\mu_{01}$ and $\mu_{12}$ and the reduced detuning energy $\left(\mathrm{E}_{1}-\mathrm{E}_{2} / 2\right)$. 
Table S11. Comparison of calculated and experimental key NMR resonances in bisbtz-I and its rhenium complex $\left({ }^{15} \mathrm{~N}\right.$ NMR shifts wrt. to $\mathrm{CH}_{3} \mathrm{NO}_{2},{ }^{1} \mathrm{H}$ NMR shifts wrt. to TMS). Chemical shieldings are decomposed into diamagnetic, paramagnetic and spin-orbit contributions.

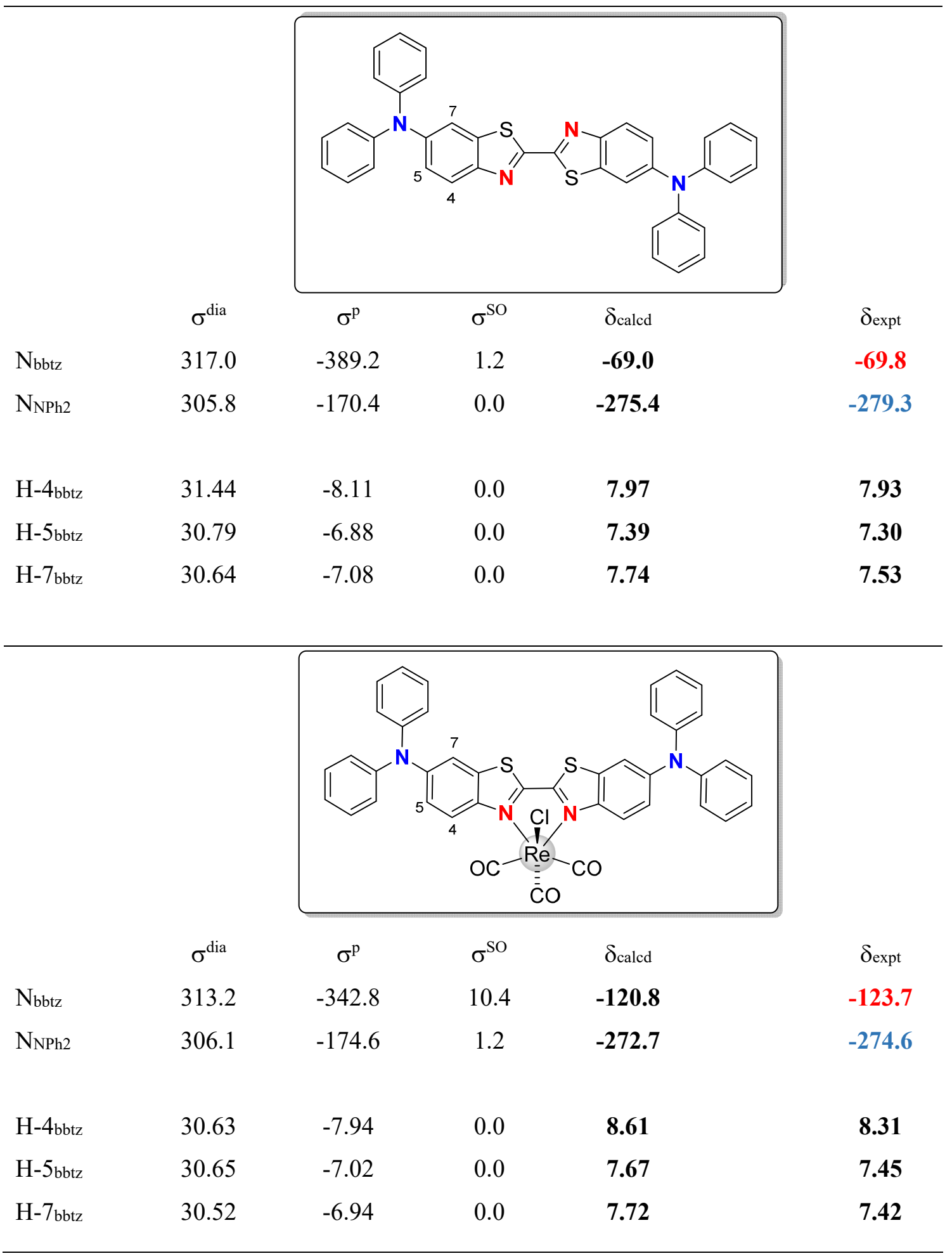

${ }^{a}$ Calculations done at the 2c-ZORA(SO)/TZ2P level (cf. Computational details). 


\section{Cartesian coordinates of pertinent DFT optimized structures}

\begin{tabular}{|c|c|c|c|}
\hline \multicolumn{4}{|c|}{ bisbtz-I } \\
\hline & 0.7149608 & 0.0939836 & -0.0035178 \\
\hline & 1.7788130 & -1.2900368 & 0.0047899 \\
\hline & 3.1202689 & -0.2049355 & 0.0003900 \\
\hline & 2.6477823 & 1.1241957 & -0.0076484 \\
\hline & 1.2877067 & 1.2552757 & -0.0076871 \\
\hline & 3.5723614 & 2.1709972 & -0.0122061 \\
\hline & 4.9160038 & 1.8835278 & -0.0011204 \\
\hline & 5.3809385 & 0.5543991 & -0.0013392 \\
\hline & 4.4751779 & -0.4993008 & -0.0082807 \\
\hline & 3.2177938 & 3.1942522 & -0.0060335 \\
\hline & 5.6423630 & 2.6861460 & 0.0125970 \\
\hline & 4.8317958 & -1.5211798 & -0.0124509 \\
\hline & -0.7149608 & -0.0939836 & -0.0035178 \\
\hline & -1.7788130 & 1.2900368 & 0.0047899 \\
\hline & -3.1202689 & 0.2049355 & 0.0003900 \\
\hline & -2.6477823 & -1.1241957 & -0.0076484 \\
\hline & -1.2877067 & -1.2552757 & -0.0076871 \\
\hline & -3.5723614 & -2.1709972 & -0.0122061 \\
\hline & -4.9160038 & -1.8835278 & -0.0011204 \\
\hline & -5.3809385 & -0.5543991 & -0.0013392 \\
\hline & -4.4751779 & 0.4993008 & -0.0082807 \\
\hline & -3.2177938 & -3.1942522 & -0.0060335 \\
\hline & -5.6423630 & -2.6861460 & 0.0125970 \\
\hline & -4.8317958 & 1.5211798 & -0.0124509 \\
\hline & -6.7598912 & -0.3006302 & 0.0184215 \\
\hline & -7.2664851 & 0.7816738 & 0.7632746 \\
\hline & -8.2628099 & 1.5988900 & 0.2341137 \\
\hline & -8.7624790 & 2.6566533 & 0.9749530 \\
\hline & -8.2685048 & 2.9250280 & 2.2430138 \\
\hline & -7.2714509 & 2.1153673 & 2.7685222 \\
\hline & -6.7773851 & 1.0466476 & 2.0412212 \\
\hline & -7.6350791 & -1.0950400 & -0.7446057 \\
\hline & -8.8804394 & -1.4587231 & -0.2343633 \\
\hline & -9.7418812 & -2.2359670 & -0.9888268 \\
\hline & -9.3733935 & -2.6751484 & -2.252738 \\
\hline & -8.1319845 & -2.3190817 & -2.7590677 \\
\hline & -7.2701614 & -1.5282485 & -2.0180384 \\
\hline & -8.6434558 & 1.3959792 & -0.7595203 \\
\hline & -9.5364057 & 3.2848696 & 0.5490220 \\
\hline & -8.6569702 & 3.7574724 & 2.8170269 \\
\hline & -6.8817035 & 2.3083165 & 3.7614372 \\
\hline & -6.0060909 & 0.4095055 & 2.4568051 \\
\hline & -9.1662455 & -1.1253237 & 0.7557494 \\
\hline & -10.7060988 & -2.5122172 & -0.5777633 \\
\hline & -10.0478857 & -3.2887830 & -2.8374240 \\
\hline & -7.8334590 & -2.6475659 & -3.7479683 \\
\hline & -6.3062965 & -1.2416542 & -2.4206818 \\
\hline & 6.7598912 & 0.3006302 & 0.0184215 \\
\hline & 7.2664851 & -0.7816738 & 0.7632746 \\
\hline & 8.2628099 & -1.5988900 & 0.2341137 \\
\hline & 8.7624790 & -2.6566533 & 0.9749530 \\
\hline & 8.2685048 & -2.9250280 & 2.2430138 \\
\hline
\end{tabular}




$\begin{array}{rrrr}\text { C } & 7.2714509 & -2.1153673 & 2.7685222 \\ \mathrm{C} & 6.7773851 & -1.0466476 & 2.0412212 \\ \mathrm{C} & 7.6350791 & 1.0950400 & -0.7446057 \\ \mathrm{C} & 8.8804394 & 1.4587231 & -0.2343633 \\ \mathrm{C} & 9.7418812 & 2.2359670 & -0.9888268 \\ \mathrm{C} & 9.3733935 & 2.6751484 & -2.2527384 \\ \mathrm{C} & 8.1319845 & 2.3190817 & -2.7590677 \\ \mathrm{C} & 7.2701614 & 1.5282485 & -2.0180384 \\ \mathrm{H} & 8.6434558 & -1.3959792 & -0.7595203 \\ \mathrm{H} & 9.5364057 & -3.2848696 & 0.5490220 \\ \mathrm{H} & 8.6569702 & -3.7574724 & 2.8170269 \\ \mathrm{H} & 6.8817035 & -2.3083165 & 3.7614372 \\ \mathrm{H} & 6.0060909 & -0.4095055 & 2.4568051 \\ \mathrm{H} & 9.1662455 & 1.1253237 & 0.7557494 \\ \mathrm{H} & 10.7060988 & 2.5122172 & -0.5777633 \\ \mathrm{H} & 10.0478857 & 3.2887830 & -2.8374240 \\ \mathrm{H} & 7.8334590 & 2.6475659 & -3.7479683 \\ \mathrm{H} & 6.3062965 & 1.2416542 & -2.4206818\end{array}$

90

\section{bisbtz-II}

$\begin{array}{lrrr}\text { C } & 0.7161362 & 0.0924685 & -0.0378730 \\ \mathrm{~S} & 1.7756157 & -1.2916444 & -0.0398569 \\ \mathrm{C} & 3.1187526 & -0.2084304 & -0.0403530 \\ \mathrm{C} & 2.6490493 & 1.1213258 & -0.0340022 \\ \mathrm{~N} & 1.2880757 & 1.2538806 & -0.0353936 \\ \mathrm{C} & 3.5720215 & 2.1682977 & -0.0242289 \\ \mathrm{C} & 4.9152920 & 1.8743046 & -0.0196408 \\ \mathrm{C} & 5.3896640 & 0.5477566 & -0.0240201 \\ \mathrm{C} & 4.4752128 & -0.4992620 & -0.0333510 \\ \mathrm{H} & 3.2163536 & 3.1911140 & -0.0306018 \\ \mathrm{H} & 5.6353207 & 2.6833980 & -0.0413186 \\ \mathrm{H} & 4.8221650 & -1.5248698 & -0.0056854 \\ \mathrm{C} & -0.7161362 & -0.0924685 & -0.0378730 \\ \mathrm{~S} & -1.7756157 & 1.2916444 & -0.0398569 \\ \mathrm{C} & -3.1187526 & 0.2084304 & -0.0403530 \\ \mathrm{C} & -2.6490493 & -1.1213258 & -0.0340022 \\ \mathrm{~N} & -1.2880757 & -1.2538806 & -0.0353936 \\ \mathrm{C} & -3.5720215 & -2.1682977 & -0.0242289 \\ \mathrm{C} & -4.9152920 & -1.8743046 & -0.0196408 \\ \mathrm{C} & -5.3896640 & -0.5477566 & -0.0240201 \\ \mathrm{C} & -4.4752128 & 0.4992620 & -0.0333510 \\ \mathrm{H} & -3.2163536 & -3.1911140 & -0.0306018 \\ \mathrm{H} & -5.6353207 & -2.6833980 & -0.0413186 \\ \mathrm{H} & -4.8221650 & 1.5248698 & -0.0056854 \\ \mathrm{C} & -6.8345422 & -0.2764491 & -0.0192013 \\ \mathrm{C} & 6.8345422 & 0.2764491 & -0.0192013 \\ \mathrm{C} & -7.3718598 & 0.8069440 & -0.7159496 \\ \mathrm{C} & -8.7286965 & 1.0628003 & -0.7172319 \\ \mathrm{C} & -9.6046889 & 0.2455924 & -0.0024709 \\ \mathrm{C} & -9.0781271 & -0.8362776 & 0.7031242 \\ \mathrm{C} & -7.7214386 & -1.0924267 & 0.6846588 \\ \mathrm{H} & -6.7176884 & 1.4388273 & -1.3059836 \\ \mathrm{H} & -9.1230884 & 1.8970517 & -1.2842952 \\ \mathrm{~N} & -10.9819407 & 0.5039062 & 0.0050900 \\ \mathrm{H} & -9.7424711 & -1.4721679 & 1.2751065 \\ \mathrm{H} & -7.3363181 & -1.9222154 & 1.2664662 \\ & & & \end{array}$




\begin{tabular}{|c|c|c|c|}
\hline 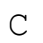 & -11.4547724 & 1.8291661 & 0.0255446 \\
\hline $\mathrm{C}$ & -12.5549200 & 2.1956049 & -0.7473777 \\
\hline C & -13.0263225 & 3.4968080 & -0.7165490 \\
\hline C & -12.4027049 & 4.4545643 & 0.0700471 \\
\hline C & -11.3028680 & 4.0928500 & 0.83452 \\
\hline $\mathrm{C}$ & -10.8342718 & 2.7902519 & 0.82157 \\
\hline $\mathrm{C}$ & -11.9005584 & -0.5625418 & -0.00067 \\
\hline C & -13.0390384 & -0.5162102 & 0.801467 \\
\hline C & -13.9448144 & -1.5629911 & 0.78687 \\
\hline$C$ & -13.7246382 & -2.6750547 & -0.012852 \\
\hline$C$ & -12.5885649 & -2.7249320 & -0.80772 \\
\hline c & -11.6853536 & -1.6759666 & -0.81048 \\
\hline $\mathrm{H}$ & -13.0376970 & 1.4512383 & -1.368 \\
\hline $\mathrm{H}$ & -13. & 3. & -1 \\
\hline $\mathrm{H}$ & -12.7 & & \\
\hline $\mathrm{H}$ & -10 & & \\
\hline $\mathrm{H}$ & -9.9825937 & 2.5076264 & 1 . \\
\hline $\mathrm{H}$ & -13.2081838 & 0.3476786 & 328 \\
\hline $\mathrm{H}$ & -14.8247529 & -1.5134759 & 1.418062 \\
\hline $\mathrm{H}$ & -14.4322738 & -3.4951587 & -0.01736 \\
\hline $\mathrm{H}$ & -12.4082043 & -3.5832927 & -1.444749 \\
\hline $\mathrm{H}$ & -10.8042726 & -1.7115480 & -1.4397276 \\
\hline C & 7.3718598 & -0.8069440 & -0.71594 \\
\hline C & 8.72869 & -1.0628003 & -0.71723 \\
\hline C & 9.6046889 & -0.2455924 & -0.00247 \\
\hline$c$ & 9.0781271 & 0.8362776 & 0.703. \\
\hline $\mathrm{C}$ & 7.7214386 & 4267 & 0 . \\
\hline $\mathrm{H}$ & 6884 & -1 & -1 \\
\hline $\mathrm{H}$ & 9.1 & -1 & -1 \\
\hline $\mathrm{N}$ & 10.9819407 & 9062 & $50 s$ \\
\hline $\mathrm{H}$ & 9.7424711 & 1.4721679 & 1.2751065 \\
\hline $\mathrm{H}$ & 7.3363181 & 1.9222154 & 1.2664662 \\
\hline $\mathrm{C}$ & 11.4547724 & -1.8291661 & 0.0255446 \\
\hline $\mathrm{C}$ & 12.5549200 & -2.1956049 & -0.747377 \\
\hline $\mathrm{C}$ & 13.0263225 & -3.4968080 & -0.7165490 \\
\hline $\mathrm{C}$ & 12.4027049 & -4.4545643 & 0.070047 \\
\hline $\mathrm{C}$ & 11.3028680 & -4.092850 & 0.834523 \\
\hline $\mathrm{C}$ & 10.8342718 & -2.7902519 & 0.821579 \\
\hline $\mathrm{C}$ & 11.9005584 & 0.5625418 & -0.000671 \\
\hline C & 13.0390384 & 0.5162102 & 0.8014 \\
\hline C & 13.9448144 & 1.5629911 & 0 . \\
\hline C & 13. & 2 . & -0 \\
\hline C & 12.5 & 20 & -0 \\
\hline C & 11.6853536 & 9666 & -0.810480 \\
\hline $\mathrm{H}$ & 13.0376970 & -1.4512383 & -1.368904 \\
\hline $\mathrm{H}$ & 13.8823367 & -3.7663418 & -1.324581 \\
\hline $\mathrm{H}$ & 12.7708299 & -5.4731521 & 0.0877687 \\
\hline $\mathrm{H}$ & 10.8110670 & -4.8282517 & 1.460950 \\
\hline $\mathrm{H}$ & 9.9825937 & -2.5076264 & 1.428364 \\
\hline $\mathrm{H}$ & 13.2081838 & -0.3476786 & 1.4328109 \\
\hline $\mathrm{H}$ & 14.8247529 & 1.5134759 & 1.4180627 \\
\hline $\mathrm{H}$ & 14.4322738 & 3.4951587 & -0.0173634 \\
\hline $\mathrm{H}$ & 12.4082043 & 3.5832927 & -1.444749 \\
\hline & & & \\
\hline
\end{tabular}


106

\section{bisbtz-III}

1. 7786418

3. 1194365

2. 6464515

1. 2852479

3. 5676519

4. 9114415

5.3895731

4.4762136

3. 2100650

5. 6296919

4.8247899

$-0.7156514$

$-1.7786418$

$-3.1194365$

$-2.6464515$

$-1.2852479$

$-3.5676519$

$-4.9114415$

$-5.3895731$

$-4.4762136$

$-3.2100650$

$-5.6296919$

$-4.8247899$

$-6.8339035$

6.8339035

$-7.3744193$

$-8.7305145$

$-9.6093133$

$-9.0776930$

$-7.7212647$

$-6.7220824$

$-9.1239252$

$-10.9807369$

$-9.7394524$

$-7.3359798$

$-11.4625574$

$-12.5447230$

$-13.0322481$

$-12.4387714$

$-11.3554582$

$-10.8851175$

$-11.9076796$

$-13.0094528$

$-13.9269612$

$-13.7589348$

$-12.6609490$

$-11.7541613$

$-13.0070959$

$-13.8728975$

$-12.9789945$

$-10.8782458$

$-10.0483203$

$-13.1434269$

$-14.7838001$

\begin{tabular}{|c|c|}
\hline 0.0942899 & -0.0656900 \\
\hline-1.2874279 & -0.0643309 \\
\hline-0.2011203 & -0.0641111 \\
\hline 1.1274418 & -0.0594704 \\
\hline 1.2569398 & -0.0627409 \\
\hline 2.1760373 & -0.0482882 \\
\hline 1.8847424 & -0.041 \\
\hline 0.5592085 & -0.04 \\
\hline-0.4891913 & -0.05 \\
\hline 3.1982239 & -0.05 \\
\hline 2.695 & -0.06 \\
\hline-1.5141538 & -0.02 \\
\hline-0.0942899 & -0.06 \\
\hline 1.28 & -0 \\
\hline 0.20 & -0.0 \\
\hline-1.12 & -0 \\
\hline-1.25 & -0.0 \\
\hline-2.17 & -0 \\
\hline-1.88 & -0.0 \\
\hline-0.55 & -0.0 \\
\hline 0.48 & -0.0 \\
\hline-3.19 & -0 . \\
\hline-2.69 & -0 \\
\hline 1.51 & -0 . \\
\hline-0.29 & -0 . \\
\hline 0.29 & -0 \\
\hline 0.79 & -0.72 \\
\hline 1.05 & -0.7 \\
\hline 0.23 & -0.0 \\
\hline-0.85 & 0.6 \\
\hline-1.11 & 0. \\
\hline 1.43 & $-1 \cdot 3$ \\
\hline 1.89 & $-1 \cdot 2$ \\
\hline $0.4 \varepsilon$ & \\
\hline-1.50 & 1.2 \\
\hline-1.94 & 1.2 \\
\hline 1.81 & -0.0 \\
\hline 2.14 & -0.86 \\
\hline 3.43 & -0.8 \\
\hline 4.43 & -0 \\
\hline 4.11 & 0.68 \\
\hline 2.81 & 0.72 \\
\hline-0.57 & 0.0 \\
\hline-0.49 & 0.9 \\
\hline-1.52 & 0.9 \\
\hline-2.66 & 0.1 \\
\hline-2.75 & -0.6 \\
\hline-1.70 & -0.7 \\
\hline 1.37 & $-1 \cdot 4$ \\
\hline 3.69 & -1.52 \\
\hline 5.67 & -0.2 \\
\hline 4.86 & 1.294 \\
\hline 2.56 & 1.3 \\
\hline 0 & \\
\hline-1.46 & 1.6 \\
\hline
\end{tabular}




$$
\begin{array}{rrr}
-14.7036297 & -3.6238952 & 0.3334583 \\
-12.5068601 & -3.6215539 & -1.2736584 \\
-10.9063601 & -1.7732542 & -1.3886908 \\
7.3744193 & -0.7993261 & -0.7212397 \\
8.7305145 & -1.0557373 & -0.7182420 \\
9.6093133 & -0.2317603 & -0.0107922 \\
9.0776930 & 0.8580622 & 0.6826089 \\
7.7212647 & 1.1124341 & 0.6598729 \\
6.7220824 & -1.4372084 & -1.3070627 \\
9.1239252 & -1.8958413 & -1.2770002 \\
10.9807369 & -0.4894701 & 0.0019248 \\
9.7394524 & 1.5008874 & 1.2495232 \\
7.3359798 & 1.9475403 & 1.2341496 \\
11.4625574 & -1.8134797 & -0.0436842 \\
12.5447230 & -2.1444798 & -0.8600167 \\
13.0322481 & -3.4333131 & -0.8961787 \\
12.4387714 & -4.4345549 & -0.1300403 \\
11.3554582 & -4.1168216 & 0.6812366 \\
10.8851175 & -2.8120841 & 0.7284462 \\
11.9076796 & 0.5706072 & 0.0686544 \\
13.0094528 & 0.4950728 & 0.9211523 \\
13.9269612 & 1.5219620 & 0.9791932 \\
13.7589348 & 2.6641575 & 0.1990641 \\
12.6609490 & 2.7519775 & -0.6490270 \\
11.7541613 & 1.7040881 & -0.7173939 \\
13.0070959 & -1.3739858 & -1.4653003 \\
13.8728975 & -3.6928068 & -1.5282007 \\
12.9789945 & -5.6708354 & -0.2400326 \\
10.8782458 & -4.8696153 & 1.2940588 \\
10.0483203 & -2.5685747 & 1.3720263 \\
13.1434269 & -0.3858336 & 1.5376226 \\
14.7838001 & 1.4663083 & 1.6397419 \\
14.7036297 & 3.6238952 & 0.3334583 \\
12.5068601 & 3.6215539 & -1.2736584 \\
10.9063601 & 1.7732542 & -1.3886908 \\
-12.4078280 & 6.7024646 & 0.5243593 \\
-12.9743723 & 7.6029508 & 0.2938184 \\
-12.4819309 & 6.4959719 & 1.5979124 \\
-11.3558799 & 6.8620971 & 0.2622640 \\
-14.5647885 & -4.7886885 & -0.4405256 \\
-15.4093999 & -5.4266495 & -0.1864878 \\
-14.5926470 & -4.5651160 & -1.5128615 \\
-13.6326590 & -5.3163243 & -0.2091461 \\
12.4078280 & -6.7024646 & 0.5243593 \\
12.9743723 & -7.6029508 & 0.2938184 \\
12.4819309 & -6.4959719 & 1.5979124 \\
11.3558799 & -6.8620971 & 0.2622640 \\
14.5647885 & 4.7886885 & -0.4405256 \\
15.4093999 & 5.4266495 & -0.1864878 \\
14.5926470 & 4.5651160 & -1.5128615 \\
13.6326590 & 5.3163243 & -0.2091461
\end{array}
$$


94

\section{bisbtz-IV}

\subsection{8}

0.6200903

2. 3452357

2.7834927

1.7854233

4.1534868

5.0446213

4.6056083

3.2408598

4.4889086

6.1099235

5.5529395

2.9057710

$-0.6236388$

$-0.6200903$

$-2.3452357$

$-2.7834927$

$-1.7854233$

$-4.1534868$

$-5.0446213$

$-4.6056083$

$-3.2408598$

$-4.4889086$

$-6.1099235$

$-5.5529395$

$-2.9057710$

$-6.3680901$

$-7.3206224$

6.3680901

7.3206224

$-6.9260356$

$-7.8541996$

$-9.2154135$

$-9.6153758$

$-8.6864001$

$-5.8748986$

$-7.5300641$

$-10.1565209$

$-10.6660123$

$-9.0104615$

$-9.8342915$

$-10.2326088$

$-9.9273956$

$-9.2107236$

$-8.8093667$

$-9.1241718$

$-11.4412061$

$-12.5667838$

$-13.8273104$

$-13.9857179$

$-12.8655305$

$-11.6003220$

$-10.7853211$

$-10.2425294$

$-8.9678741$

$$
\begin{aligned}
& -0.3630285-0.2463455 \\
& -2.1053726-0.2422480 \\
& -2.0642110 \quad-0.2287099 \\
& -0.7219378 \quad-0.2299571 \\
& 0.2102564-0.2407443 \\
& -0.4477188 \quad-0.2156251 \\
& -1.4921552-0.1972469 \\
& -2.8353005-0.1939313 \\
& -3.1207771-0.2120728 \\
& 0.5818490-0.2170106 \\
& -1.3000021-0.1837708 \\
& -3.8858443-0.1655163 \\
& -4.1498469-0.2087538 \\
& 0.3630285-0.2463455 \\
& 2.1053726-0.2422480 \\
& 2.0642110-0.2287099 \\
& 0.7219378 \quad-0.2299571 \\
& -0.2102564-0.2407443 \\
& 0.4477188 \quad-0.2156251 \\
& 1.4921552-0.1972469 \\
& 2.8353005-0.1939313 \\
& 3.1207771-0.2120728 \\
& -0.5818490-0.2170106 \\
& 1.3000021-0.1837708 \\
& 3.8858443-0.1655163 \\
& 4.1498469-0.2087538 \\
& 4.7789443-0.1327655 \\
& 5.8229991-0.0865326 \\
& -4.7789443-0.1327655 \\
& -5.8229991-0.0865326 \\
& 7.1637173-0.1679638 \\
& 8.1817005-0.1210912 \\
& \begin{array}{ll}
7.8992128 & 0.0215040
\end{array} \\
& 6.5628034 \quad 0.1058658 \\
& \begin{array}{ll}
5.5461772 & 0.0471929
\end{array} \\
& 7.3980235-0.2839089 \\
& 9.2119990 \quad-0.1964751 \\
& 8.9308310 \quad 0.0791558 \\
& 6.3284241 \quad 0.2222877 \\
& 4.5153774 \quad 0.1224234 \\
& \begin{array}{ll}
10.1580069 & 0.6917907
\end{array} \\
& \begin{array}{ll}
11.3588901 & 0.1084567
\end{array} \\
& 12.5639839 \quad 0.7179075 \\
& 12.5916851 \quad 1.9054604 \\
& 11.3963930 \quad 2.4847044 \\
& 10.1864149 \quad 1.8903254 \\
& 8.7553638-0.4730865 \\
& 9.2058147 \quad 0.2129529 \\
& 9.0463904-0.3370249 \\
& 8.4253844-1.5675404 \\
& 7.9710695-2.2488415 \\
& 8.1401186-1.7128960 \\
& 11.3384348 \quad-0.8229348 \\
& 13.4904005 \quad 0.2516478 \\
& 13.5365392 \quad 2.3762259
\end{aligned}
$$




$\begin{array}{rr}11.4026666 & 3.4175117 \\ 9.2536900 & 2.3500332 \\ 9.6840905 & 1.1771690 \\ 9.4001789 & 0.2088192 \\ 8.2970834 & -1.9925319 \\ 7.4921598 & -3.2151633 \\ 7.7956336 & -2.2507455 \\ -7.1637173 & -0.1679638 \\ -8.1817005 & -0.1210912 \\ -7.8992128 & 0.0215040 \\ -6.5628034 & 0.1058658 \\ -5.5461772 & 0.0471929 \\ -7.3980235 & -0.2839089 \\ -9.2119990 & -0.1964751 \\ -8.9308310 & 0.0791558 \\ -6.3284241 & 0.2222877 \\ -4.5153774 & 0.1224234 \\ -10.1580069 & 0.6917907 \\ -11.3588901 & 0.1084567 \\ -12.5639839 & 0.7179075 \\ -12.5916851 & 1.9054604 \\ -11.3963930 & 2.4847044 \\ -10.1864149 & 1.8903254 \\ -8.7553638 & -0.4730865 \\ -9.2058147 & 0.2129529 \\ -9.0463904 & -0.3370249 \\ -8.4253844 & -1.5675404 \\ -7.9710695 & -2.2488415 \\ -8.1401186 & -1.7128960 \\ -11.3384348 & -0.8229348 \\ -13.4904005 & 0.2516478 \\ -13.5365392 & 2.3762259 \\ -11.4026666 & 3.4175117 \\ -9.2536900 & 2.3500332 \\ -9.6840905 & 1.1771690 \\ -9.4001789 & 0.2088192 \\ -8.2970834 & -1.9925319 \\ -7.4921598 & -3.2151633 \\ -7.7956336 & -2.2507455\end{array}$

\section{0}

\section{bisbtz-V}

$\begin{array}{lrrr}\text { C } & 0.6231584 & -0.3633390 & 0.0796946 \\ \mathrm{~S} & 0.6181472 & -2.1059244 & 0.0851077 \\ \mathrm{C} & 2.3434309 & -2.0664599 & 0.0771737 \\ \mathrm{C} & 2.7827559 & -0.7245632 & 0.0707742 \\ \mathrm{~N} & 1.7856361 & 0.2086071 & 0.0733434 \\ \mathrm{C} & 4.1531698 & -0.4521663 & 0.0603306 \\ \mathrm{C} & 5.0432222 & -1.4976183 & 0.0559215 \\ \mathrm{C} & 4.6032843 & -2.8406517 & 0.0627616 \\ \mathrm{C} & 3.2377999 & -3.1239785 & 0.0741240 \\ \mathrm{H} & 4.4897721 & 0.5770402 & 0.0551784 \\ \mathrm{H} & 6.1088120 & -1.3066839 & 0.0474416 \\ \mathrm{C} & 5.5490778 & -3.8921155 & 0.0559320 \\ \mathrm{H} & 2.9015901 & -4.1526779 & 0.0782125 \\ \mathrm{C} & -0.6231584 & 0.3633390 & 0.0796946 \\ \mathrm{~S} & -0.6181472 & 2.1059244 & 0.0851077\end{array}$




\begin{tabular}{|c|c|c|c|}
\hline $\mathrm{C}$ & -2.3434309 & 2.0664599 & 0.0771737 \\
\hline $\mathrm{C}$ & -2.7827559 & 0.7245632 & 0.0707742 \\
\hline $\mathrm{N}$ & -1.7856361 & -0.2086071 & 0.0733434 \\
\hline $\mathrm{C}$ & -4.1531698 & 0.4521663 & 0.0603306 \\
\hline$C$ & -5.0432222 & 1.4976183 & 0.0559215 \\
\hline C & -4.6032843 & 2.8406517 & 0.0627616 \\
\hline & -3.2377999 & 3.1239785 & 0.0741240 \\
\hline $\mathrm{H}$ & -4.4897721 & -0.5770402 & 0.0551784 \\
\hline & -6.1088120 & 1.3066839 & 0.0474416 \\
\hline C & -5.5490778 & 3.8921155 & 0.0559320 \\
\hline $\mathrm{H}$ & -2.9015901 & 4.1526779 & 0.0782125 \\
\hline c & -6.3628522 & 4.7874680 & 0.0472866 \\
\hline c & -7.3139650 & 5.8325237 & 0.0341519 \\
\hline $\mathrm{C}$ & 6.3628522 & -4.7874680 & 0.0472866 \\
\hline C & 7.3139650 & -5.8325237 & 0.03 \\
\hline $\mathrm{C}$ & -6.9180465 & 7.1726944 & 0.12 \\
\hline C & -7.8441529 & 8.1925297 & 0.1 \\
\hline C & -9.2124981 & 7.9153554 & 0.0 \\
\hline C & -9.6135239 & 6.5768038 & -0.0 \\
\hline c & -8.6835756 & 5.5605825 & -0.0 \\
\hline $\mathrm{H}$ & -5.8626783 & 7.4067502 & 0.1 \\
\hline $\mathrm{H}$ & -7.5134897 & 9.2216084 & 0.1 \\
\hline $\mathrm{N}$ & -10.1496908 & 8.9430923 & -0.0 \\
\hline $\mathrm{H}$ & -10.6676574 & 6.3415771 & -0.1 \\
\hline $\mathrm{H}$ & -9.0129768 & 4.5302353 & -0.1 \\
\hline C & -9.9000736 & 10.1631127 & 0.6 \\
\hline $\mathrm{C}$ & -10.1732463 & 11.3773020 & 0.01 \\
\hline C & -9.9526588 & 12.5726239 & 0.66 \\
\hline $\mathrm{C}$ & -9.4354303 & 12.5872283 & 36312 \\
\hline C & -9.1548829 & 11.3819469 & 2.5 \\
\hline $\mathrm{C}$ & -9.4002422 & 10.1831456 & 1.9436270 \\
\hline $\mathrm{C}$ & -11.3769050 & 8.7902002 & -0.6967291 \\
\hline $\mathrm{C}$ & -12.572 & 9.1749556 & -0.0 \\
\hline $\mathrm{C}$ & -13.7724734 & 9.0464816 & -0.7568090 \\
\hline $\mathrm{C}$ & -13.8116122 & 8.5125055 & -2.0436212 \\
\hline $\mathrm{C}$ & -12.6250623 & 8.1221357 & -2.6548 \\
\hline $\mathrm{C}$ & -11.4197892 & 8.2749710 & -1.9845844 \\
\hline $\mathrm{H}$ & -10.5700847 & 11.3738579 & -0.9889144 \\
\hline $\mathrm{H}$ & -10.1641617 & 13.5179193 & 0.1 \\
\hline O & -9.2425492 & 13.8070557 & 2.5156752 \\
\hline $\mathrm{H}$ & -8.7603238 & 11.3600448 & 3.6047756 \\
\hline $\mathrm{H}$ & -9.1915384 & 9.2463445 & 2.4465915 \\
\hline $\mathrm{H}$ & -12.5505032 & 9.5850859 & 0.9122106 \\
\hline $\mathrm{H}$ & -14.7036376 & 9.3443975 & -0.290 \\
\hline O & -15.0350756 & 8.4136995 & -2.6121629 \\
\hline $\mathrm{H}$ & -12.6214704 & 7.7123825 & -3.6 \\
\hline $\mathrm{H}$ & -10.4964838 & 7.9801584 & -2.4690890 \\
\hline $\mathrm{C}$ & 6.9180465 & -7.1726944 & 0.1 \\
\hline $\mathrm{C}$ & 7.8441529 & -8.1925297 & 0.1 \\
\hline $\mathrm{C}$ & 9.212 & -7.9153554 & 0.0 \\
\hline $\mathrm{C}$ & 9.6135239 & -6.5768038 & -0.0818875 \\
\hline $\mathrm{C}$ & 8.6835756 & -5.5605825 & -0.0689768 \\
\hline $\mathrm{H}$ & 5.8626783 & -7.4067502 & 0.1921193 \\
\hline $\mathrm{H}$ & 7.5134897 & -9.2216084 & 0.1632863 \\
\hline $\mathrm{N}$ & 10.1496908 & -8.9430923 & -0.01 \\
\hline 11 & 10.66 & -6.3415771 & -0.1 \\
\hline$-T$ & 9.0129768 & -4.5302353 & -0.1 \\
\hline
\end{tabular}




$\begin{array}{rrrr}\mathrm{C} & 9.9000736 & -10.1631127 & 0.6491182 \\ \mathrm{C} & 10.1732463 & -11.3773020 & 0.0192107 \\ \mathrm{C} & 9.9526588 & -12.5726239 & 0.6694792 \\ \mathrm{C} & 9.4354303 & -12.5872283 & 1.9636312 \\ \mathrm{C} & 9.1548829 & -11.3819469 & 2.5980350 \\ \mathrm{C} & 9.4002422 & -10.1831456 & 1.9436270 \\ \mathrm{C} & 11.3769050 & -8.7902002 & -0.6967291 \\ \mathrm{C} & 12.5725705 & -9.1749556 & -0.0903585 \\ \mathrm{C} & 13.7724734 & -9.0464816 & -0.7568090 \\ \mathrm{C} & 13.8116122 & -8.5125055 & -2.0436212 \\ \mathrm{C} & 12.6250623 & -8.1221357 & -2.6548912 \\ \mathrm{C} & 11.4197892 & -8.2749710 & -1.9845844 \\ \mathrm{H} & 10.5700847 & -11.3738579 & -0.9889144 \\ \mathrm{H} & 10.1641617 & -13.5179193 & 0.1846892 \\ \mathrm{O} & 9.2425492 & -13.8070557 & 2.5156752 \\ \mathrm{H} & 8.7603238 & -11.3600448 & 3.6047756 \\ \mathrm{H} & 9.1915384 & -9.2463445 & 2.4465915 \\ \mathrm{H} & 12.5505032 & -9.5850859 & 0.9122106 \\ \mathrm{H} & 14.7036376 & -9.3443975 & -0.2903709 \\ \mathrm{O} & 15.0350756 & -8.4136995 & -2.6121629 \\ \mathrm{H} & 12.6214704 & -7.7123825 & -3.6558151 \\ \mathrm{H} & 10.4964838 & -7.9801584 & -2.4690890 \\ \mathrm{C} & -8.7263086 & 13.8592617 & 3.8220886 \\ \mathrm{H} & -8.6452621 & 14.9146484 & 4.0760159 \\ \mathrm{H} & -9.3937162 & 13.3671229 & 4.5380887 \\ \mathrm{H} & -7.7345403 & 13.3974654 & 3.8809618 \\ \mathrm{C} & -15.1108944 & 7.8848181 & -3.9123149 \\ \mathrm{H} & -16.1655379 & 7.8877792 & -4.1816575 \\ \mathrm{H} & -14.5545747 & 8.4987757 & -4.6290845 \\ \mathrm{H} & -14.7321689 & 6.8575277 & -3.9508039 \\ \mathrm{C} & 8.7263086 & -13.8592617 & 3.8220886 \\ \mathrm{H} & 8.6452621 & -14.9146484 & 4.0760159 \\ \mathrm{H} & 9.3937162 & -13.3671229 & 4.5380887 \\ \mathrm{H} & 7.7345403 & -13.3974654 & 3.8809618 \\ \mathrm{C} & 15.1108944 & -7.8848181 & -3.9123149 \\ \mathrm{H} & 16.1655379 & -7.8877792 & -4.1816575 \\ \mathrm{H} & 14.5545747 & -8.4987757 & -4.6290845 \\ \mathrm{H} & 14.7321689 & -6.8575277 & -3.9508039\end{array}$

98

\section{bisbtz-VI}

$\begin{array}{lrrr}\mathrm{S} & 1.3005046 & 1.7688692 & -0.1051933 \\ \mathrm{C} & -0.0883750 & 0.7158162 & -0.1043622 \\ \mathrm{~N} & -1.2475008 & 1.2957504 & -0.1025125 \\ \mathrm{C} & -2.1508410 & 3.5847537 & -0.0994286 \\ \mathrm{C} & -1.8555272 & 4.9244425 & -0.0975383 \\ \mathrm{C} & -0.5242733 & 5.3979328 & -0.0952560 \\ \mathrm{C} & 0.5169408 & 4.4724738 & -0.1006109 \\ \mathrm{C} & 0.2242473 & 3.1170876 & -0.1020941 \\ \mathrm{C} & -1.1071262 & 2.6536141 & -0.1005839 \\ \mathrm{H} & -3.1748216 & 3.2321736 & -0.1022659 \\ \mathrm{H} & -2.6706144 & 5.6369799 & -0.1031242 \\ \mathrm{H} & 1.5437281 & 4.8200267 & -0.1009226 \\ \mathrm{C} & -0.1896892 & 6.8116709 & -0.0876589 \\ \mathrm{H} & 0.8757740 & 7.0218573 & -0.1278316 \\ \mathrm{C} & -1.0489899 & 7.8399104 & -0.0249675 \\ \mathrm{H} & -2.1133462 & 7.6297399 & 0.0391410\end{array}$




$$
\begin{aligned}
& -0.7193788 \\
& 9.2530469-0.0193590 \\
& 0.5786319 \\
& 0.8369203 \\
& -0.1960894 \\
& -1.4942129 \\
& -1.7424950 \\
& 1.4041144 \\
& 1.8486705 \\
& -2.3080370 \\
& -2.7597224 \\
& 0.0693917 \\
& -0.8630878 \\
& -1.1398264 \\
& -2.0490687 \\
& -2.7049765 \\
& -2.4336199 \\
& -1.5147450 \\
& -0.6341924 \\
& -2.2555491 \\
& -3.4207103 \\
& -2.9315003 \\
& -1.2959514 \\
& 1.2671599 \\
& 1.7627645 \\
& 2.9445185 \\
& 3.6399596 \\
& \text { 3. } 1421194 \\
& 1.9690024 \\
& 1.2151168 \\
& 3.3168485 \\
& 4.5613383 \\
& 3.6783356 \\
& 1.5854225 \\
& -1.3005046 \\
& 0.0883750 \\
& \text { 1. } 2475008 \\
& 2.1508410 \\
& 1.8555272 \\
& 0.5242733 \\
& -0.5169408 \\
& -0.2242473 \\
& 1.1071262 \\
& 3.1748216 \\
& \text { 2. } 6706144 \\
& -1.5437281 \\
& 0.1896892 \\
& -0.8757740 \\
& 1.0489899 \\
& 2.1133462 \\
& 0.7193788 \\
& -0.5786319 \\
& -0.8369203 \\
& 0.1960894 \\
& 1.4942129 \\
& 1.7424950 \\
& -1.4041144 \\
& -1.8486705 \\
& 9.7521056-0.1826298 \\
& 11.1048364-0.1686639 \\
& 12.0288665 \quad 0.0203419 \\
& 11.5468742 \quad 0.1837655 \\
& 10.1892112 \quad 0.1551088 \\
& 9.0697637-0.3474625 \\
& 11.4638793-0.3106188 \\
& 12.2454000 \quad 0.3329963 \\
& 9.8361727 \quad 0.2894901 \\
& 13.4027905 \quad 0.0372121 \\
& 14.3137598 \quad-0.4955081 \\
& 15.5062443 \quad 0.1694104 \\
& 16.4051882-0.3612970 \\
& 16.1248246-1.5510614 \\
& 14.9352570-2.2117830 \\
& 14.0379273-1.6958376 \\
& 15.7225925 \quad 1.1027971 \\
& 17.3278910 \quad 0.1688528 \\
& 16.8273634-1.9603483 \\
& 14.7074674-3.1473648 \\
& 13.1144832-2.2182935 \\
& 13.8877656 \quad 0.5965324 \\
& \begin{array}{ll}
13.3515672 & 1.7836687
\end{array} \\
& \begin{array}{ll}
13.8297472 & 2.3239687
\end{array} \\
& 14.8563977 \quad 1.7013416 \\
& \begin{array}{lr}
15.3977262 & 0.5246527
\end{array} \\
& 14.9155783-0.0303578 \\
& 12.5578358 \quad 2.2768463 \\
& 13.4040213 \quad 3.2486761 \\
& 15.2318261 \quad 2.1295905 \\
& 16.1956314 \quad 0.0239512 \\
& 15.3327935-0.9534080 \\
& -1.7688692-0.1051933 \\
& -0.7158162-0.1043622 \\
& -1.2957504-0.1025125 \\
& -3.5847537-0.0994286 \\
& -4.9244425-0.0975383 \\
& -5.3979328-0.0952560 \\
& -4.4724738-0.1006109 \\
& -3.1170876-0.1020941 \\
& \begin{array}{ll}
-2.6536141 & -0.1005839
\end{array} \\
& -3.2321736-0.1022659 \\
& -5.6369799-0.1031242 \\
& -4.8200267-0.1009226 \\
& -6.8116709-0.0876589 \\
& -7.0218573-0.1278316 \\
& -7.8399104 \quad-0.0249675 \\
& \begin{array}{ll}
-7.6297399 & 0.0391410
\end{array} \\
& -9.2530469-0.0193590 \\
& -9.7521056-0.1826298 \\
& \begin{array}{ll}
-11.1048364 & -0.1686639
\end{array} \\
& \begin{array}{ll}
-12.0288665 & 0.0203419
\end{array} \\
& \begin{array}{ll}
-11.5468742 & 0.1837655
\end{array} \\
& \begin{array}{ll}
-10.1892112 & 0.1551088
\end{array} \\
& -9.0697637-0.3474625 \\
& -11.4638793-0.3106188
\end{aligned}
$$




$\begin{array}{rrrr}\text { H } & 2.3080370 & -12.2454000 & 0.3329963 \\ \mathrm{H} & 2.7597224 & -9.8361727 & 0.2894901 \\ \mathrm{~N} & -0.0693917 & -13.4027905 & 0.0372121 \\ \mathrm{C} & -1.2671599 & -13.8877656 & 0.5965324 \\ \mathrm{C} & -1.7627645 & -13.3515672 & 1.7836687 \\ \mathrm{C} & -2.9445185 & -13.8297472 & 2.3239687 \\ \mathrm{C} & -3.6399596 & -14.8563977 & 1.7013416 \\ \mathrm{C} & -3.1421194 & -15.3977262 & 0.5246527 \\ \mathrm{C} & -1.9690024 & -14.9155783 & -0.0303578 \\ \mathrm{H} & -1.2151168 & -12.5578358 & 2.2768463 \\ \mathrm{H} & -3.3168485 & -13.4040213 & 3.2486761 \\ \mathrm{H} & -4.5613383 & -15.2318261 & 2.1295905 \\ \mathrm{H} & -3.6783356 & -16.1956314 & 0.0239512 \\ \mathrm{H} & -1.5854225 & -15.3327935 & -0.9534080 \\ \mathrm{C} & 0.8630878 & -14.3137598 & -0.4955081 \\ \mathrm{C} & 1.1398264 & -15.5062443 & 0.1694104 \\ \mathrm{C} & 2.0490687 & -16.4051882 & -0.3612970 \\ \mathrm{C} & 2.7049765 & -16.1248246 & -1.5510614 \\ \mathrm{C} & 2.4336199 & -14.9352570 & -2.2117830 \\ \mathrm{C} & 1.5147450 & -14.0379273 & -1.6958376 \\ \mathrm{H} & 0.6341924 & -15.7225925 & 1.1027971 \\ \mathrm{H} & 2.2555491 & -17.3278910 & 0.1688528 \\ \mathrm{H} & 3.4207103 & -16.8273634 & -1.9603483 \\ \mathrm{H} & 2.9315003 & -14.7074674 & -3.1473648 \\ \mathrm{H} & 1.2959514 & -13.1144832 & -2.2182935\end{array}$

50

\section{btz-VI}

$\begin{array}{lrrr}\text { S } & 1.4594513 & -8.5951129 & -0.2677318 \\ \mathrm{C} & 0.0823477 & -9.6483955 & -0.1944671 \\ \mathrm{~N} & -1.0709774 & -9.0846096 & -0.1061821 \\ \mathrm{C} & -1.9770627 & -6.7927929 & -0.0036794 \\ \mathrm{C} & -1.6889566 & -5.4504479 & 0.0009431 \\ \mathrm{C} & -0.3639256 & -4.9693983 & -0.0783138 \\ \mathrm{C} & 0.6779466 & -5.8884718 & -0.1650291 \\ \mathrm{C} & 0.3891503 & -7.2454788 & -0.1706247 \\ \mathrm{C} & -0.9333835 & -7.7176118 & -0.0902362 \\ \mathrm{H} & -2.9973995 & -7.1507453 & 0.0574162 \\ \mathrm{H} & -2.5060580 & -4.7430871 & 0.0663325 \\ \mathrm{H} & 1.7010673 & -5.5358010 & -0.2269602 \\ \mathrm{C} & -0.0330585 & -3.5533871 & -0.0742922 \\ \mathrm{H} & 1.0305441 & -3.3421364 & -0.1459893 \\ \mathrm{C} & -0.8903689 & -2.5262146 & 0.0116259 \\ \mathrm{H} & -1.9534640 & -2.7356965 & 0.0961885 \\ \mathrm{C} & -0.5592415 & -1.1124588 & 0.0112370 \\ \mathrm{C} & 0.7389635 & -0.6156340 & -0.1554885 \\ \mathrm{C} & 0.9985808 & 0.7370057 & -0.1495508 \\ \mathrm{C} & -0.0333021 & 1.6626462 & 0.0347023 \\ \mathrm{C} & -1.3313868 & 1.1832126 & 0.2029413 \\ \mathrm{C} & -1.5809613 & -0.1746439 & 0.1827258 \\ \mathrm{H} & 1.5640920 & -1.2997721 & -0.3147615 \\ \mathrm{H} & 2.0107864 & 1.0943546 & -0.2930873 \\ \mathrm{H} & -2.1442980 & 1.8836591 & 0.3489247 \\ \mathrm{H} & -2.5982270 & -0.5263985 & 0.3204048 \\ \mathrm{~N} & 0.2340764 & 3.0368903 & 0.0431783 \\ \mathrm{C} & -0.6908598 & 3.9438851 & -0.5078835 \\ \mathrm{C} & -0.9694866 & 5.1452326 & 0.1400777\end{array}$




$\begin{array}{lrrr}\mathrm{C} & -1.8718489 & 6.0399631 & -0.4089752 \\ \mathrm{C} & -2.5194259 & 5.7466860 & -1.6001212 \\ \mathrm{C} & -2.2467042 & 4.5480982 & -2.2435861 \\ \mathrm{C} & -1.3343049 & 3.6548845 & -1.7095144 \\ \mathrm{H} & -0.4712670 & 5.3717538 & 1.0751000 \\ \mathrm{H} & -2.0797292 & 6.9699364 & 0.1079204 \\ \mathrm{H} & -3.2299448 & 6.4461337 & -2.0237178 \\ \mathrm{H} & -2.7383220 & 4.3098199 & -3.1799517 \\ \mathrm{H} & -1.1142016 & 2.7242754 & -2.2186584 \\ \mathrm{C} & 1.4279893 & 3.5226634 & 0.6092950 \\ \mathrm{C} & 1.9100519 & 2.9943609 & 1.8055031 \\ \mathrm{C} & 3.0889447 & 3.4714583 & 2.3527506 \\ \mathrm{C} & 3.7950677 & 4.4893463 & 1.7278997 \\ \mathrm{C} & 3.3108999 & 5.0227223 & 0.5420071 \\ \mathrm{C} & 2.1407184 & 4.5414832 & -0.0198891 \\ \mathrm{H} & 1.3538879 & 2.2074523 & 2.3002449 \\ \mathrm{H} & 3.4506164 & 3.0519604 & 3.2846025 \\ \mathrm{H} & 4.7142592 & 4.8641005 & 2.1615179 \\ \mathrm{H} & 3.8558135 & 5.8136184 & 0.0394076 \\ \mathrm{H} & 1.7684760 & 4.9521810 & -0.9505541 \\ \mathrm{H} & 0.2244346 & -10.7214892 & -0.2237012\end{array}$

92

\section{bpy-VI}

$\begin{array}{rrrr}\mathrm{C} & 0.5612895 & 0.4840364 & -0.2059182 \\ \mathrm{~N} & 0.2471984 & 1.7796919 & -0.2259950 \\ \mathrm{C} & 1.2493251 & 2.6506723 & -0.2251460 \\ \mathrm{C} & 2.5847088 & 2.2979355 & -0.2019313 \\ \mathrm{C} & 2.9291071 & 0.9429361 & -0.1776019 \\ \mathrm{C} & 1.8754887 & 0.0321823 & -0.1821092 \\ \mathrm{H} & 0.9678709 & 3.7001899 & -0.2432989 \\ \mathrm{H} & 3.3390138 & 3.0745818 & -0.2006029 \\ \mathrm{C} & 4.2952665 & 0.4451375 & -0.1435151 \\ \mathrm{H} & 2.0561771 & -1.0350228 & -0.1660821 \\ \mathrm{C} & -0.5612895 & -0.4840364 & -0.2059182 \\ \mathrm{C} & -1.8754887 & -0.0321823 & -0.1821092 \\ \mathrm{C} & -2.9291071 & -0.9429361 & -0.1776019 \\ \mathrm{C} & -2.5847088 & -2.2979355 & -0.2019313 \\ \mathrm{C} & -1.2493251 & -2.6506723 & -0.2251460 \\ \mathrm{~N} & -0.2471984 & -1.7796919 & -0.2259950 \\ \mathrm{H} & -2.0561771 & 1.0350228 & -0.1660821 \\ \mathrm{C} & -4.2952665 & -0.4451375 & -0.1435151 \\ \mathrm{H} & -3.3390138 & -3.0745818 & -0.2006029 \\ \mathrm{H} & -0.9678709 & -3.7001899 & -0.2432989 \\ \mathrm{C} & 5.4082458 & 1.1916907 & -0.1392875 \\ \mathrm{C} & 6.7794366 & 0.7181259 & -0.0988462 \\ \mathrm{C} & 7.8199229 & 1.6498063 & -0.0530708 \\ \mathrm{C} & 9.1443188 & 1.2636843 & 0.0025049 \\ \mathrm{C} & 9.4833458 & -0.0889773 & 0.0019435 \\ \mathrm{C} & 8.4527331 & -1.0334223 & -0.0560783 \\ \mathrm{C} & 7.1349723 & -0.6362324 & -0.1004799 \\ \mathrm{H} & 7.5779002 & 2.7076225 & -0.0443092 \\ \mathrm{H} & 9.9273866 & 2.0101789 & 0.0485783 \\ \mathrm{~N} & 10.8207997 & -0.4960008 & 0.0539231 \\ \mathrm{H} & 8.7010550 & -2.0874623 & -0.0691026 \\ \mathrm{H} & 6.3661027 & -1.3977678 & -0.1551081 \\ \mathrm{C} & 11.8192234 & 0.2613862 & -0.5890997\end{array}$




\begin{tabular}{|c|c|c|c|}
\hline $\mathrm{C}$ & 13.0390483 & 0.4971040 & 0.0411402 \\
\hline $\mathrm{C}$ & 14.0230651 & 1.2314659 & -0.5984508 \\
\hline C & 13.8025220 & 1.7528803 & -1.8649493 \\
\hline $\mathrm{C}$ & 12.5857302 & 1.5237931 & -2.491093 \\
\hline $\mathrm{C}$ & 11.6021701 & 0.7777251 & -1.86490 \\
\hline C & 11.1840499 & -1.6537260 & 0.76821 \\
\hline C & 12.1356625 & -2.5293207 & 0.24945 \\
\hline C & 12.5007227 & -3.6608760 & 0.95805 \\
\hline$C$ & 11.9147932 & -3.9452086 & 2.18306 \\
\hline$C$ & 10.9627795 & -3.0772528 & 2.69776 \\
\hline$C^{\prime}$ & 10.6036836 & -1.9349398 & 2.00332 \\
\hline H & 13.2096227 & 0.0968602 & 1.0332 \\
\hline $\mathrm{H}$ & 14.5 & 1.40784 & -0 . \\
\hline $\mathrm{H}$ & 14. & & -2 \\
\hline $\mathrm{H}$ & 12. & & \\
\hline $\mathrm{H}$ & 10.6566648 & 0.5901121 & -2.3593826 \\
\hline $\mathrm{H}$ & 12.5877297 & -2.3128820 & -0 \\
\hline $\mathrm{H}$ & 13.2415715 & -4.3333269 & 0.5409 \\
\hline $\mathrm{H}$ & 12.1980609 & -4.8349913 & 2.73211 \\
\hline $\mathrm{H}$ & 10.5028349 & -3.2822656 & 3.657710 \\
\hline $\mathrm{H}$ & 9.8687009 & -1.2524983 & 2.4124776 \\
\hline $\mathrm{C}$ & -5.4082458 & -1.1916907 & -0.1392875 \\
\hline C & -6.7794366 & -0.7181259 & -0.0988462 \\
\hline C & -7.1349723 & 0.6362324 & -0.10047 \\
\hline C & -8.4527331 & 1.0334223 & -0.05607 \\
\hline C & -9.4833458 & $0.08897^{\circ}$ & 0.0019 \\
\hline C & -9.1443188 & -1.26368 & 0 . \\
\hline$c$ & -7.815 & -1 & -0 \\
\hline $\mathrm{H}$ & -6.3 & 1 & -0 \\
\hline $\mathrm{H}$ & -8.7010550 & 2.0874623 & -0.0691026 \\
\hline $\mathrm{N}$ & -10.8207997 & 0.4960008 & 0.0539231 \\
\hline $\mathrm{H}$ & -9.9273866 & -2.0101789 & 0.0485783 \\
\hline $\mathrm{H}$ & -7.5779002 & -2.7076225 & -0.0443092 \\
\hline $\mathrm{C}$ & -11.8192234 & -0.2613862 & -0.5890997 \\
\hline $\mathrm{C}$ & -13.0390483 & -0.4971040 & 0.0411402 \\
\hline $\mathrm{C}$ & -14.0230651 & -1.2314659 & -0.5984508 \\
\hline $\mathrm{C}$ & -13.8025220 & -1.7528803 & -1.864949 \\
\hline C & -12.5857302 & -1.5237931 & -2.4910930 \\
\hline $\mathrm{C}$ & -11.6021701 & -0.7777251 & -1.864901 \\
\hline C & -11.1840499 & 1.65372 & 0.7682 \\
\hline $\mathrm{C}$ & -12 & 2 . & \\
\hline C & -12 & 0 & \\
\hline C & -11.9147932 & 36 & 30605 \\
\hline $\mathrm{C}$ & -10.9627795 & 3.0772528 & 7768 \\
\hline $\mathrm{C}$ & -10.6036836 & 1.9349398 & 2.0033276 \\
\hline $\mathrm{H}$ & -13.2096227 & -0.0968602 & 1.0332249 \\
\hline $\mathrm{H}$ & -14.9666306 & -1.4078439 & -0.0948106 \\
\hline $\mathrm{H}$ & -14.5724114 & -2.3319685 & -2.3601852 \\
\hline $\mathrm{H}$ & -12.4029927 & -1.9171864 & -3.4845183 \\
\hline $\mathrm{H}$ & -10.6566648 & -0.5901121 & -2.3593826 \\
\hline $\mathrm{H}$ & -12.5877297 & 2.3128820 & -0.7109334 \\
\hline $\mathrm{H}$ & -13.2415715 & 4.3333269 & 0.5409602 \\
\hline $\mathrm{H}$ & -12.1980609 & 4.834991 & 2.732118 \\
\hline $\mathrm{H}$ & -10.5028349 & 3.282265 & 3.6577104 \\
\hline $\mathrm{H}$ & 87009 & 24983 & 2.4124776 \\
\hline $\mathrm{H}$ & 4.3792076 & -0.6375390 & -0.114362 \\
\hline 14 & 5.3085035 & 2.2741283 & -0.163292 \\
\hline
\end{tabular}




$\begin{array}{llrr}\mathrm{H} & -4.3792076 & 0.6375390 & -0.1143622 \\ \mathrm{H} & -5.3085035 & -2.2741283 & -0.1632925\end{array}$

\section{8}

\section{ReCl (CO) 3_bisbtz-I}

\begin{tabular}{|c|c|c|c|}
\hline $\mathrm{Re}$ & $-1.1 \overline{5} 12168$ & 2.2843472 & 0.0000000 \\
\hline $\mathrm{Cl}$ & -3.1396378 & 0.8535360 & 0.0000000 \\
\hline C & -1.8800478 & 3.4775905 & 1.3051994 \\
\hline 0 & -2.3167538 & 4.2057999 & 2.0794113 \\
\hline C & 0.4641429 & 3.3074034 & 0.0000000 \\
\hline 0 & 1.4304597 & 3.9359294 & 0.0000000 \\
\hline $\mathrm{C}$ & -1.8800478 & 3.4775905 & -1.3051994 \\
\hline 0 & -2.3167538 & 4.2057999 & -2.0794113 \\
\hline S & 0.4551901 & -1.6983254 & 1.7328607 \\
\hline $\mathrm{C}$ & -0.0101959 & -0.3928152 & 0.712678 \\
\hline $\mathrm{N}$ & -0.3711739 & 0.7082094 & 1.332765 \\
\hline $\mathrm{C}$ & -0.2885094 & 0.5683622 & 2.693868 \\
\hline $\mathrm{C}$ & -0.5839689 & 1.5284531 & 3.6628788 \\
\hline $\mathrm{C}$ & -0.4437518 & 1.2062455 & 4.9888809 \\
\hline $\mathrm{C}$ & 0.0031485 & -0.0662621 & 5.4025626 \\
\hline $\mathrm{C}$ & 0.3101849 & -1.0294203 & 4.4459065 \\
\hline $\mathrm{C}$ & 0.1513244 & -0.7013762 & 3.1114947 \\
\hline $\mathrm{H}$ & -0.9384728 & 2.5064380 & 3.3721065 \\
\hline $\mathrm{H}$ & -0.6915153 & 1.9407932 & 5 . \\
\hline $\mathrm{H}$ & 0.65 & -2.0078249 & 4 . \\
\hline $\mathrm{S}$ & 0.455 & -1.6983254 & -1 \\
\hline C & -0.0101959 & -0.3928152 & -0.7126785 \\
\hline $\mathrm{N}$ & -0.3711739 & 0.7082094 & -1.3327659 \\
\hline $\mathrm{C}$ & -0.2885094 & 0.5683622 & -2.6938680 \\
\hline C & -0.5839689 & 1.5284531 & -3.6628788 \\
\hline $\mathrm{C}$ & -0.4437518 & 1.2062455 & -4.9888809 \\
\hline $\mathrm{C}$ & 0.0031485 & -0.0662621 & -5.4025626 \\
\hline $\mathrm{C}$ & 0.3101849 & -1.0294203 & -4.4459065 \\
\hline $\mathrm{C}$ & 0.1513244 & -0.7013762 & -3.1114947 \\
\hline $\mathrm{H}$ & -0.9384728 & 2.5064380 & -3.3721065 \\
\hline $\mathrm{H}$ & -0.6915153 & 1.9407932 & -5.7437432 \\
\hline $\mathrm{H}$ & 0.659 & -2.0078249 & -4.7467323 \\
\hline $\mathrm{N}$ & 0.1237414 & -0.3529887 & 6.7596255 \\
\hline $\mathrm{C}$ & -0.0706552 & -1.6679985 & 7.2350333 \\
\hline $\mathrm{C}$ & -1.1619055 & -2.4191137 & 044898 \\
\hline C & -1.3486933 & -3.7061148 & 7.2785739 \\
\hline $\mathrm{C}$ & -0.4632280 & -4.2535135 & 8.1965863 \\
\hline $\mathrm{C}$ & 0.6176641 & -3.5015922 & 8.6327278 \\
\hline $\mathrm{C}$ & 0.8203527 & -2.2187603 & 8.1519444 \\
\hline $\mathrm{H}$ & -1.8614094 & -1.9867319 & 6.0989214 \\
\hline $\mathrm{H}$ & -2.2036390 & -4.2792454 & 6.939161 \\
\hline $\mathrm{H}$ & -0.6172482 & -5.2579245 & 8.571742 \\
\hline $\mathrm{H}$ & 1.3179564 & -3.9192551 & 9.3466646 \\
\hline $\mathrm{H}$ & 1.6664313 & -1.6318684 & 8.4887444 \\
\hline $\mathrm{C}$ & 0.5030888 & 0.6531994 & 7.6742035 \\
\hline $\mathrm{C}$ & -0.1328585 & 0.7449368 & 8.9098036 \\
\hline $\mathrm{C}$ & 0.2465966 & 1.7214438 & 9.8146479 \\
\hline $\mathrm{C}$ & 1.2508997 & 2.6247297 & 9.4958272 \\
\hline $\mathrm{C}$ & 1.8813765 & 2.5360569 & 8.2632364 \\
\hline $\mathrm{C}$ & 1.5194679 & 1.5516369 & 7.3588470 \\
\hline $\mathrm{H}$ & -0.9224752 & 0.0446065 & 9.1538192 \\
\hline $\mathrm{H}$ & -0.2573667 & 1.7844784 & 10.7719720 \\
\hline
\end{tabular}




$\begin{array}{rrrr}\mathrm{H} & 1.5401803 & 3.3920993 & 10.2033780 \\ \mathrm{H} & 2.6727046 & 3.2302748 & 8.0057990 \\ \mathrm{H} & 2.0217491 & 1.4751689 & 6.4019227 \\ \mathrm{~N} & 0.1237414 & -0.3529887 & -6.7596255 \\ \mathrm{C} & -0.0706552 & -1.6679985 & -7.2350333 \\ \mathrm{C} & -1.1619055 & -2.4191137 & -6.8044898 \\ \mathrm{C} & -1.3486933 & -3.7061148 & -7.2785739 \\ \mathrm{C} & -0.4632280 & -4.2535135 & -8.1965863 \\ \mathrm{C} & 0.6176641 & -3.5015922 & -8.6327278 \\ \mathrm{C} & 0.8203527 & -2.2187603 & -8.1519444 \\ \mathrm{H} & -1.8614094 & -1.9867319 & -6.0989214 \\ \mathrm{H} & -2.2036390 & -4.2792454 & -6.9391611 \\ \mathrm{H} & -0.6172482 & -5.2579245 & -8.5717423 \\ \mathrm{H} & 1.3179564 & -3.9192551 & -9.3466646 \\ \mathrm{H} & 1.6664313 & -1.6318684 & -8.4887444 \\ \mathrm{C} & 0.5030888 & 0.6531994 & -7.6742035 \\ \mathrm{C} & -0.1328585 & 0.7449368 & -8.9098036 \\ \mathrm{C} & 0.2465966 & 1.7214438 & -9.8146479 \\ \mathrm{C} & 1.2508997 & 2.6247297 & -9.4958272 \\ \mathrm{C} & 1.8813765 & 2.5360569 & -8.2632364 \\ \mathrm{C} & 1.5194679 & 1.5516369 & -7.3588470 \\ \mathrm{H} & -0.9224752 & 0.0446065 & -9.1538192 \\ \mathrm{H} & -0.2573667 & 1.7844784 & -10.7719720 \\ \mathrm{H} & 1.5401803 & 3.3920993 & -10.2033780 \\ \mathrm{H} & 2.6727046 & 3.2302748 & -8.0057990 \\ \mathrm{H} & 2.0217491 & 1.4751689 & -6.4019227\end{array}$




\section{References}

[1] Harris, R. K.; Becker, E. D.; Cabral de Menezes, S. M.; Goodfellow, R.; Granger, P. Pure and Applied Chemistry 2001, 73, 1795.

[2] Hunter, R. F.; Parken, E. R. J. Chem. Soc. 1935, 1935, 1755-1761.

[3] Hrobarikova, V.; Hrobarik, P.; Gajdos, P.; Fitilis, I.; Fakis, M.; Persephonis, P.; Zahradnik, P. J. Org. Chem. 2010, 75, 3053-3068.

[4] Hrobarik, P.; Hrobarikova, V.; Sigmundova, I.; Zahradnik, P.; Fakis, M.; Polyzos, I.; Persephonis, P. J. Org. Chem. 2011, 76, 8726-8736.

[5] Xu, F.; Peng, L.; Orita, A.; Otera, J. Org. Lett. 2012, 14, 3970-3973.

[6] Modha, S. G.; Popescu, M. V.; Greaney, M. F. J. Org. Chem. 2017, 82, 11933-11938.

[7] Lambert, C.; Nöll, G.; Schmälzlin, E.; Meerholz, K.; Bräuchle, C. Chem. Eur. J. 1998, 4, 2129-2135.

[8] Tydlitat, J.; Feckova, M.; Le Poul, P.; Pytela, O.; Klikar, M.; Rodríguez-López, J.; Robin-le Guen, F.; Achelle, S. Eur. J. Org. Chem. 2019, 2019, 1921-1930.

[9] Li, Z.; Ye, T.; Tang, S.; Wang, C.; Ma, D.; Li, Z. J. Mater. Chem. C 2015, 3, 2016-2023.

[10] Wei, H.; Feng, R.; Fang, Y.; Wang, L.; Chen, C.; Zhang, L.; Cui, H.; Wang, X. Chem. Eur. J. 2018, 24, 19341-19347.

[11] Bielawski, M.; Zhu, M.; Olofsson, B. Adv. Synth. Catal. 2007, 349, 2610.

[12] Tian, Z.-Y.; Ming, X.-X.; Teng, H.-B.; Hu, Y.-T.; Zhang, C.-P. Chem. Eur. J. 2018, 24, 13744-13748.

[13] Miloudi, A.; El-Abed, D.; Boyer, G.; Finet, J.; Galy, J.; Siri, D. Eur. J. Org. Chem. 2004, 2004, 1509-1516.

[14] Vera, M. D.; Pelletier, J. C. J. Comb. Chem. 2007, 9, 569.

[15] Williams, A. T. R.; Winfield, S. A.; Miller, J. N. Analyst 1983, 108, 1067-1071.

[16] Berlman, I. B. (1971). Fluorescence Spectra of Aromatic Molecules, $2^{\text {nd }}$ Ed. New York and London: Academic Press. p. 399.

[17] Seintis, K.; Şahin, Ç.; Sigmundová, I.; Stathatos, E.; Hrobárik, P.; Fakis, M. J. Phys. Chem. C 2018, 122, 20122-20134.

[18] Xu, C.; Webb, W. W. J. Opt. Soc. Am. B 1996, 13, 481-491.

[19] Fitilis, I.; Fakis, M.; Polyzos, I.; Giannetas, V.; Persephonis, P.; Vellis, P.; Mikroyannidis, J. Chem. Phys. Lett. 2007, 447, 300-304.

[20] Makarov, N. S.; Drobizhev, M.; Rebane, A. Opt. Express 2008, 16, 4029-4047.

[21] Sheldrick, G. M., SHELXT - Integrated space-group and crystal-structure determination. Acta Crystallogr. Sect. A 2015, 71, 3-8. 
[22] Sheldrick, G. M., Crystal structure refinement with SHELXL. Acta Crystallogr. Sect. C 2015, 71, 3-8.

[23] Spek, A. L., PLATON SQUEEZE: a tool for the calculation of the disordered solvent contribution to the calculated structure factors. Acta Crystallogr. Sect. C 2015, 71, 9-18.

[24] Perdew, J. P.; Burke, K.; Ernzerhof, M. Phys. Rev. Lett. 1996, 77, 3865-3868.

[25] Adamo, C.; Barone, V. Chem. Phys. Lett. 1998, 298, 113-119.

[26] Weigend, F.; Ahlrichs, R. Phys. Chem. Chem. Phys. 2005, 7, 3297-3305.

[27] Grimme, S.; Antony, J.; Ehrlich, S.; Krieg, H. J. Chem. Phys. 2010, 132, 154104.

[28] Grimme, S.; Ehrlich, S.; Goerigk, L. J. Comput. Chem. 2011, 32, 1456-1465.

[29] TURBOMOLE, version 7.5; TURBOMOLE GmBH, a development of the University of Karlsruhe and Forschungszentrum Karlsruhe, 2020; available from: http://www.turbomole.com.

[30] Wolff, S. K.; Ziegler, T. J. Chem. Phys., 1998, 109, 895-905.

[31] Wolff, S. K.; Ziegler, T; van Lenthe, E.; Baerends, E. J. J. Chem. Phys. 1999, 110, 76897698.

[32] Amsterdam Density Functional (ADF), version 2017.112, SCM, Theoretical Chemistry, Vrije Univeriteit, Amsterdam, Netherlands, 2018; available from http://www.scm.com.

[33] te Velde, G.; Bickelhaupt, F. M.; Baerends, E. J.; Guerra, C. F.; Van Gisbergen, S. J.; Snijders, J. G.; Ziegler, T. J. Comput. Chem. 2001, 22, 931-967.

[34] Hansen, A. E.; Bouman, T. D. J. Chem. Phys. 1985, 82, 5035-5047.

[35] Yanai, T.; Tew, D. P.; Handy, N. C. Chem. Phys. Lett. 2004, 393, 51-57.

[36] DALTON, a molecular electronic structure program, release Dalton2020, 2020; available from http://daltonprogram.org.

[37] Norman, P.; Ruud, K., Microscopic theory of nonlinear optics. In Nonlinear optical properties of matter: From molecules to condensed phases, Springer, Netherlands, 2006; pp $1-49$.

[38] Salek, P.; Vahtras, O.; Guo, J. D.; Luo, Y.; Helgaker, T.; Agren, H. Chem. Phys. Lett. 2003, $374,446-452$.

[39] Rudberg, E.; Salek, P.; Helgaker, T.; Agren, H. J. Chem. Phys. 2005, 123, 184108.

[40] Zareba, J. K.; Nyk, M.; Samoc, M. Polymers 2020, 12, 1670.

[41] Jin, F.; Rong, Z.; Guo, X.; Ma, M.; Lv, J.; Zhang, L.; Liao, R.; Liu, Y.; Tao, D.; Tian, Y. Dyes Pigm. 2018, 150, 174-180.

[42] Durand, N.; Mhanna, R.; Savel, P.; Akdas-Kiliç, H.; Malval, J.-P.; Soppera, O.; Fillaut, J.L. Chem. Comm. 2020, 56, 12801-12804. 